\title{
Testes para Hipóteses Restritas em Desigualdades Lineares \\ Usando Equações de Estimação Generalizadas
}

\author{
José Cardoso Neto
}

Tese apresentada

\author{
ao \\ Instituto de Matemática e Estatística \\ da \\ Universidade de São Paulo \\ para \\ obtenção do grau \\ de \\ Doutor em Estatística
}

Área de concentração: Estatística

Orientador: Professor Dr. Gilberto Alvarenga Paula 


\title{
Testes para Hipóteses Restritas em Desigualdades Lineares Usando Equações de Estimação Generalizadas
}

\author{
Este exemplar corresponde à redação \\ final da tese devidamente corrigida \\ e defendida por José Cardoso Neto \\ e aprovada pela comissão julgadora.
}

São Paulo, 06 de abril de 2000.

Banca examinadora:

- Prof. Dr. Gilberto Alvarenga Paula (Orientador)

IME/USP

- Prof. Dr. Heleno Bolfarine

IME/USP

- Prof. Dr. Josemar Rodrigues

UFSCar

- Prof. Dr. Enrico Colosimo UFMG

- Prof. Dr. Gauss Moutinho Cordeiro

UFBA 
Pelo privilégio de conviver de maneira alegre, saudável e harmoniosa com minha esposa Ângela Líbia e com meus filhos Cardosinho e Flavinho, com muito amor e carinho dedico a eles este trabalho. 


\section{Agradecimentos}

- À senhora Julia Martins Rego que mais uma vez foi fundamental dedicando muito carinho e atenção a mim, a minha esposa e aos meus filhos, me dando tranquilidade para realizar este trabalho.

- Ao Professor Dr. Gilberto Alvarenga Paula pela sua segura orientação, ensinamentos e pelo incentivo desde o início da minha vida acadêmica.

- Aos membros da Banca Examinadora pelos elogios, críticas e pelas valiosas sugestões para futuros trabalhos.

- Aos professores e funcionarios do IME/USP pelo aprendizado durante esses anos de convívio.

- Aos professores do Departamento de Estatística da Universidade do Amazonas pelo apoio e incentivo, em particular aos professores Maria Ivanilde, José Raimundo, Celso Rômulo, Edmilson e Amazoneida que viram de perto meu empenho nesse período.

- Aos colegas da Pós-graduação do IME/USP pela agradável convivência e momentos de descontração, especialmente ao Chicão, Chiquinho, Antonieta, Eliana e respectivas famílias.

- Ao meu pai (e mãe in memorian) e sogra (e sogro in memorian) que certamente têm grande participação nesta conquista.

- À CAPES pelo apoio financeiro. 


\section{Resumo}

O objetivo deste trabalho é apresentar, inicialmente, uma unificação dos principais artigos sobre testes para hipóteses restritas envolvendo o Modelo Normal Linear. Nesse caso, a relação entre as estatísticas do teste bem como a distribuição nula assintótica das estatísticas são apresentadas nos casos em que a matriz de covariância do erro é conhecida e desconhecida. Apresentamos uma breve revisão dos Modelos Lineares Generalizados e formalmente as Equações de Estimação Generalizadas (EEGs) de Liang e Zeger (1986). É estudado, em particular, o procedimento proposto por Kodde e Palm (1986) juntamente com os resultados de Wolak (1991) para desenvolver testes para hipóteses restritas em desigualdades lineares usando as EEGs. Apresentamos também, uma extensão para EEGs do procedimento proposto por Follmann (1996) para testar hipóteses restritas usando a estatística $T^{2}$ de Hotteling. Para os procedimentos propostos, vários estudos de simulação são desenvolvidos para verificar o comportamento dos mesmos em termos de poder com relação ao teste tradicional de Wald para hipóteses não restritas. Observa-se um razoável ganho de poder dos testes de Kodde e Palm e Follmann em relação ao teste de Wald e um pequeno ganho de poder do teste de Kodde e Palm em relação ao teste de Follmann. Este último é fácil de ser aplicado e evita o cálculo dos pesos exigidos pelo teste de Kodde e Palm, o que o torna um forte competidor principalmente quando o número de restrições é maior que 4. 


\begin{abstract}
The aim of this work is to present, initially, a unification of the main results on tests for restricted hypotheses involving the Linear Normal Model. In this case, the relationship among the statistical tests as well as their asymptotic null distribution are presented for the cases of covariance matrix known and unknown. We presente a brief revision of Generalized Linear Models and formally the Generalized Estimating Equations (GEEs) of Liang e Zeger (1986). It is studied, in particular, the procedure proposed by Kodde e Palm (1986) together with the results of Wolak (1991) to develop tests for restricted hypotheses under linear inequalities using GEEs. We also presented, an extension for GEEs of the procedure proposed by Follmann (1996) to test restricted hypotheses using the $T^{2}$ - Hotteling statistic. For the proposed procedures, several simulation studies are performed to verify the behavior of their empirical powers with respect to the Wald test for unrestricted hypotheses. It is observed a reasonable gain of power for the tests of Kodde and Palm and Follmann with respect to the Wald test and a small gain of power for the Kodde and Palm test in relation to the Follmann test. This last procedure though has the advantage of bein easy to be applied, sence it avoids the calculation of the weights demanded by the Kodde and Palm test, what makes it, mainly, a strong competitor when the number of restrictions is larger than 4 .
\end{abstract}




\section{Conteúdo}

1 Introdução 1

2 Modelo Linear Geral $\quad 8$

2.1 Introdução . . . . . . . . . . . . . . . . . . 8

2.2 Modelo de regressão normal linear . . . . . . . . . . . . . . . . . . . 9

2.3 Estimação com parâmetros restritos . . . . . . . . . . . . . . . . 10

2.4 Estatísticas equivalentes . . . . . . . . . . . . . . . . . 16

2.5 Distribuição nula da estatística $\xi_{r v}$ quando $\Sigma$ é conhecida . . . . . . . 21

$2.6 \Sigma$ desconhecida . . . . . . . . . . . . . . . . . . . . 30

2.6.1 Definição das estatísticas . . . . . . . . . . . . . . . . . 30

2.6.2 Distribuição nula assintótica das estatísticas . . . . . . . . . . 35 
2.7 Aplicações . . . . . . . . . . . . . . . . . . . . . . . . . . . 38

2.7.1 Pré-teste pós-teste . . . . . . . . . . . . . . 38

2.7.2 Assinaturas de TV a cabo . . . . . . . . . . . . 40

2.7.3 Demanda de energia elétrica . . . . . . . . . . . . . 43

3 Equações de Estimação Generalizadas $\quad 47$

3.1 Introdução . . . . . . . . . . . . . . . . . . . 47

3.2 Modelos lineares generalizados . . . . . . . . . . . . . . . . . 48

3.2 .1 Definição ....................... 50

3.2.2 Casos particulares. . . . . . . . . . . . . 51

3.2.3 Estatísticas suficientes e ligações canônicas . . . . . . . . . . 52

3.2.4 Função desvio . . . . . . . . . . . . . . . . . . . . 53

3.2.5 Estimação dos parâmetros . . . . . . . . . . . . . 56

3.2.6 Estimação com parâmetros restritos . . . . . . . . . . . . 59

3.3 Hipóteses em desigualdades lineares . . . . . . . . . . . . . . . . . 62

3.4 Lema de Wolak . . . . . . . . . . . . . . . . . . 66

3.5 Equações de estimação generalizadas . . . . . . . . . . . . . . . 70 
3.5 .1 g Grupos . . . . . . . . . . . . . . . . . . 77

3.6 Estatística tipo Wald . . . . . . . . . . . . . . . . . . . . 78

3.6 .1 g Grupos . . . . . . . . . . . . . . . . . . . . 83

3.7 Aplicações . . . . . . . . . . . . . . . . . . . . . . . . . . . . 86

3.7.1 Processo infeccioso pulmonar . . . . . . . . . . . 86

3.7.2 Pacientes com ataque epiléptico . . . . . . . . . . . 90

3.7 .3 Suplemento para redução de pólipos . . . . . . . . . . . . . . . 92

4 Estudos de Simulação $\quad 95$

4.1 Introdução . . . . . . . . . . . . . . . . . . . . . . . 95

4.2 Distribuição binomial . . . . . . . . . . . . . . . . . . . . 97

4.3 Distribuição de Poisson . . . . . . . . . . . . . . . . . . . . . . 104

4.4 Distribuição gama . . . . . . . . . . . . . . . . . . . . . . . . 111

4.5 Binomial com regressores . . . . . . . . . . . . . . . . . . . . . 118

4.6 Poisson com regressores . . . . . . . . . . . . . . . . . . 125

4.7 Gama com regressores . . . . . . . . . . . . . . . . . . . . 132 
5.1 Introdução . . . . . . . . . . . . . . . . . . . . . . 139

5.2 Teste de Follmann $\ldots \ldots \ldots \ldots$. . . . . . . . . . . . . . . . . 139

5.3 Aplicações . . . . . . . . . . . . . . . . . . . . . . . . . 142

5.3 .1 Previsão da safra de maçãs . . . . . . . . . . . . . . . . . . . . 142

5.3 .2 Pressão arterial . . . . . . . . . . . . . . . . . . . . . 144

5.4 Estudos de simulação . . . . . . . . . . . . . . . . . . . . . . 145

6 Contribuições, conclusões e propostas para futuras pesquisas $\quad 157$

$\begin{array}{ll}\text { A Pesos } & 161\end{array}$

A.1 Introdução . . . . . . . . . . . . . . . . . . . . . 161

A.2 Definições . . . . . . . . . . . . . . . . . . . . . 162

A.3 Expressões para os pesos . . . . . . . . . . . . . . . . 163

B Geração de variáveis correlacionadas $\quad 167$

B.1 Variáveis binárias . . . . . . . . . . . . . . . . . . 167

B.2 Variáveis Poisson e gama . . . . . . . . . . . . . . . 170

$\begin{array}{ll}\text { C Nota sobre o poder } & 173\end{array}$ 
C.1 Situação I . . . . . . . . . . . . . . . . . . . . . . . . . . . . . . . . 173

C.2 Situação II . . . . . . . . . . . . . . . . . . . . . . . . . . . . . . . . . . 174

D Conjuntos de dados

D.1 Assinaturas de TV a cabo . . . . . . . . . . . . . . . . 176

D.2 Demanda de energia elétrica . . . . . . . . . . . . . . . 178

D.3 Processo infeccioso pulmonar . . . . . . . . . . . . . . . 180

D.4 Tratamento de epilepsia . . . . . . . . . . . . . . . . 182

D.5 Tratamento de pólipos . . . . . . . . . . . . . . . . . 183

D.6 Previsão da safra de maçãs . . . . . . . . . . . . . . . . . . . . . . . . . 184

Bibliografia 


\section{Lista de Tabelas}

2.7.1 Escores de um pré-teste pós-teste realizado em Espanhol para 20 professores. . . . . . . . . . . . . . . . . .

2.7.2 Estimativas não restritas com o respectivo desvio padrão dos parâmetros do modelo para explicar o número de assinantes de TV a cabo. . . . . . 41

2.7.3 Estimativas dos parâmetros do modelo para explicar a demanda de eletricidade agregada per capita. . . . . . . . . . . . . . . . . . 44

2.7.4 Pesos e níveis descritivos das estatísticas dos testes sobre os parâmetros do modelo para demanga de eletricidade agregada per capita . . . . . .

3.2.1 Ligação canônica para os modelos mais conhecidos e outras ligações. . . 54

3.2.2 Função desvio para os modelos mais conhecidos. . . . . . . . . . . . . 55

3.7.3 Estimativas não restritas e restritas com o respectivo desvio padrão assintótico dos parâmetros do modelo para o processo infeccioso pulmonar 
3.7.4 Estimativas e estatísticas do teste para os dados do tratamento de pólipos com vitaminas $\mathrm{C}$ e $\mathrm{E}$ mais fibra de trigo. . . . . . . . . . . . . 93

4.2.1 Binomial: Kolmogorov-Smirnov (P-valor) . . . . . . . . . . . . . . 98

4.2.2 Binomial $n=20$ : poder das estatísticas $\xi_{k p}$ e $\xi_{w} \ldots \ldots \ldots 1$

4.2.3 Binomial $n=50$ : poder das estatísticas $\xi_{k p}$ e $\xi_{w} \ldots \ldots \ldots 2$

4.2.4 Binomial $n=100$ : poder das estatísticas $\xi_{k p}$ e $\xi_{w} \ldots \ldots \ldots 3$

4.3.1 Poisson: Kolmogorov-Smirnov (P-valor) . . . . . . . . . . . . . . 105

4.3.2 Poisson $n=20$ : poder das estatísticas $\xi_{k p}$ e $\xi_{w} \ldots \ldots \ldots$. . . . . 108

4.3.3 Poisson $n=50$ : poder das estatísticas $\xi_{k p}$ e $\xi_{w} \ldots \ldots \ldots$. . . . . . . 109

4.3.4 Poisson $n=100$ : poder das estatísticas $\xi_{k p}$ e $\xi_{w} \ldots \ldots \ldots \ldots$

4.4.1 Gama: Kolmogorov-Smirnov (P-valor) . . . . . . . . . . . . . . . 112

4.4.2 Gama $n=20$ : poder das estatísticas $\xi_{k p}$ e $\xi_{w} \ldots \ldots \ldots . \ldots 115$

4.4.3 Gama $n=50$ : poder das estatísticas $\xi_{k p}$ e $\xi_{w} \ldots \ldots \ldots 116$

4.4.4 Gama $n=100$ : poder das estatísticas $\xi_{k p}$ e $\xi_{w} \ldots \ldots \ldots . \ldots 117$

4.5.1 Binomial com regressores: Kolmogorov-Smirnov (P-valor). . . . . . . . . . 119

4.5.2 Binomial com regressores $n=20$ : poder das estatísticas $\xi_{k p}$ e $\xi_{w} \ldots \ldots 122$ 
4.5.3 Binomial com regressores $n=50$ : poder das estatísticas $\xi_{k p}$ e $\xi_{w} \ldots \ldots 123$

4.5.4 Binomial com regressores $n=100$ : poder das estatísticas $\xi_{k p}$ e $\xi_{w} \ldots \ldots 124$

4.6.1 Poisson com regressores: Kolmogorov-Smirnov (P-valor) . . . . . . . . . 126

4.6.2 Poisson com regressores $n=20$ : poder das estatísticas $\xi_{k p}$ e $\xi_{w} \ldots \ldots 129$

4.6.3 Poisson com regressores $n=50$ : poder das estatísticas $\xi_{k p}$ e $\xi_{w} \ldots \ldots 130$

4.6.4 Poisson com regressores $n=100$ : poder das estatísticas $\xi_{k p}$ e $\xi_{w} \ldots \ldots 131$

4.7.1 Gama com regressores: Kolmogorov-Smirnov (P-valor). . . . . . . . . . 133

4.7.2 Gama com regressores $n=20$ : poder das estatísticas $\xi_{k p}$ e $\xi_{w} \ldots \ldots 136$

4.7.3 Gama com regressores $n=50$ : poder das estatísticas $\xi_{k p}$ e $\xi_{w} \ldots \ldots 137$

4.7.4 Gama com regressores $n=100$ : poder das estatísticas $\xi_{k p}$ e $\xi_{w} \ldots \ldots 138$

5.3.1 Estimativas dos coeficientes do modelo para previsão da safra de maçãs com o respectivo desvio padrão. . . . . . . . . . . . . . . . . . 143

5.3.2 Redução na pressão arterial em sete experimentos clínicos. . . . . . . . . 144

5.4.1 Binomial com regressores $n=20$ : poder das estatísticas $\xi_{k p}, \xi_{w}^{*}$ e $\xi_{w}^{+} \ldots 148$

5.4.2 Binomial com regressores $n=50$ : poder das estatísticas $\xi_{k p}, \xi_{w}^{*}$ e $\xi_{w}^{+} \ldots 149$

5.4.3 Binomial com regressores $n=100$ : poder das estatísticas $\xi_{k p}, \xi_{w}^{*}$ e $\xi_{w}^{+} \quad . \quad 150$ 
5.4.4 Poisson com regressores $n=20$ : poder das estatísticas $\xi_{k p}, \xi_{w}^{*}$ e $\xi_{w}^{+} \ldots 151$

5.4.5 Poisson com regressores $n=50$ : poder das estatísticas $\xi_{k p}, \xi_{w}^{*}$ e $\xi_{w}^{+} \ldots 152$

5.4.6 Poisson com regressores $n=100$ : poder das estatísticas $\xi_{k p}, \xi_{w}^{*}$ e $\xi_{w}^{+} \ldots 153$

5.4.7 Gama com regressores $n=20$ : poder das estatísticas $\xi_{k p}, \xi_{w}^{*}$ e $\xi_{w}^{+} \ldots \ldots 154$

5.4.8 Gama com regressores $n=50$ : poder das estatísticas $\xi_{k p}, \xi_{w}^{*}$ e $\xi_{w}^{+} \ldots . \quad 155$

5.4.9 Gama com regressores $n=100$ : poder das estatísticas $\xi_{k p}, \xi_{w}^{*}$ e $\xi_{w}^{+} \ldots 156$

D.1.1 Assinaturas de TV a cabo . . . . . . . . . . . . . . 177

D.2.1 Demanda de energia elétrica . . . . . . . . . . . . . . . . 178

D.3.1 Processo infeccioso pulmonar . . . . . . . . . . . . . . . . . . 180

D.4.1 Tratamento de epilepsia . . . . . . . . . . . . . . . . . . . 182

D.5.1 Tratamento de pólipos . . . . . . . . . . . . . . . . . . . 183

D.6.1 Previsão da safra de maçãs . . . . . . . . . . . . . . . . . . . . . . . . 184 


\section{Lista de Figuras}

4.2.1 Binomial $n=20$ : distribuições empírica e teórica da estatística $\xi_{k p}$ com estrutura de correlação simétrica. . . . . . . . . . . . . . . . . . . . . . . 99

4.2.2 Binomial $n=50$ : distribuições empírica e teórica da estatística $\xi_{k p}$ com estrutura de correlação simétrica. . . . . . . . . . . . . . . . . . . . . 99

4.2.3 Binomial $n=100$ : distribuições empírica e teórica da estatística $\xi_{k p}$ com estrutura de correlação simétrica. . . . . . . . . . . . . . . . . . 100

4.3.1 Poisson $n=20$ : distribuições empírica e teórica da estatística $\xi_{k p}$ com estrutura de correlação simétrica. . . . . . . . . . . . . . . . . . . . 106

4.3.2 Poisson $n=50$ : distribuições empírica e teórica da estatística $\xi_{k p}$ com estrutura de correlação simétrica. . . . . . . . . . . . . . . . 106

4.3.3 Poisson $n=100$ : distribuições empírica e teórica da estatística $\xi_{k p}$ com estrutura de correlação simétrica. . . . . . . . . . . . . . . . . . . 107

4.4.1 Gama $n=20$ : distribuições empírica e teórica da estatística $\xi_{k p}$ com estrutura de correlação simétrica. . . . . . . . . . . . . . . . . . . . . . . . 113 
4.4.2 Gama $n=50$ : distribuições empírica e teórica da estatística $\xi_{k p}$ com estrutura de correlação simétrica. . . . . . . . . . . . . . . . . . . . . . . . . 113

4.4.3 Gama $n=100$ : distribuições empírica e teórica da estatística $\xi_{k p}$ com estrutura de correlação simétrica. . . . . . . . . . . . . . . . . . . . . . . . . . 114

4.5.1 Binomial com regressores $n=20$ : distribuições empírica e teórica da estatística $\xi_{k p}$ com estrutura de correlação simétrica.

4.5.2 Binomial com regressores $n=50$ : distribuições empírica e teórica da estatística $\xi_{k p}$ com estrutura de correlação simétrica.

4.5.3 Binomial com regressores $n=100$ : distribuições empírica e teórica da estatística $\xi_{k p}$ com estrutura de correlação simétrica. . . . . . . . . . . . . 12

4.6.1 Poisson com regressores $n=20$ : distribuições empírica e teórica da estatística $\xi_{k p}$ com estrutura de correlação AR(1). . . . . . . . . . . . . . . 127

4.6.2 Poisson com regressores $n=50$ : distribuições empírica e teórica da estatística $\xi_{k p}$ com estrutura de correlação AR(1). . . . . . . . . . . . . . . . 127

4.6.3 Poisson com regressores $n=100$ : distribuições empírica e teórica da estatística $\xi_{k p}$ com estrutura de correlação $\mathrm{AR}(1) \ldots \ldots \ldots$

4.7.1 Gama com regressores $n=20$ : distribuições empírica e teórica da estatística $\xi_{k p}$ com estrutura de correlação AR(1).

4.7.2 Gama com regressores $n=50$ : distribuições empírica e teórica da estatística $\xi_{k p}$ com estrutura de correlação AR(1). 
4.7.3 Gama com regressores $n=100$ : distribuições empírica e teórica da estatística $\xi_{k p}$ com estrutura de correlação $\mathrm{AR}(1) \ldots \ldots \ldots 135$ 


\section{Capítulo 1}

\section{Introdução}

O problema de estimar parâmetros de modelos estatísticos quando os mesmos devem satisfazer algum tipo de restrição e o problema de testar hipóteses sob restrições em desigualdades lineares, aparecem com certa frequência na literatura estatística, principalmente em ensaios clínicos e econometria. Existem, por exemplo, muitas situações práticas para as quais um modelo de regressão com restrições é apropriado, devido a informações parciais disponíveis sobre os coeficientes do modelo. Como descrito em Neter et al. (1996, p. 316), em um modelo de regressão linear para explicar o gasto com lazer $(y)$ em função da renda familiar $\left(x_{1}\right)$ e da idade do chefe da família $\left(x_{2}\right)$, parece ser bastante razoável esperar que o gasto com lazer cresça com a renda familiar e portanto, levando-se em conta essa informação a priori, o coeficiente de $x_{1}$ não deve ser negativo. O modelo de regressão restrito fica, então, dado por

$$
y_{i}=\beta_{0}+\beta_{1} x_{1 i}+\beta_{2} x_{2 i}+\varepsilon_{i} \text { sujeito a } \beta_{1} \geq 0
$$

Outro exemplo interesante é apresentado por Chen e Deely (1996) sobre a previsão da 
safra de maçãs na Nova Zelândia: a NZAPB (New Zeleand Apple and Pear Marketing Bord) é o orgão estatal que, entre outras atribuições, organiza e negocia todos os contratos de exportação de maçãs na Nova Zelândia. Esse orgão registra a cada ano e para cada produtor, a produção total de maçãs e o número de árvores em cada idade para cada variedade de maçã. A idade das árvores varia de 2 a 11 anos, sendo que a idade de 11 anos significa que a árvore tem 11 anos ou mais e é considerada uma árvore madura. O primeiro ano não é considerado porque a produção é nula. Esses dados são cuidadosamente atualizados a cada ano e contêm duas informações importantes para cada produtor e cada variedade: o número de árvores em cada idade e o total de frutos. Usando esses dados, Chen e Deely (1996) construiram um modelo para prever a colheita total de maçãs de qualquer variedade.

Para reduzir a variabilidade dos dados, foi usado o índice de produtividade definido por IP: número médio de caixas por árvore e idade média das árvores. Essencialmente, esse índice cobre fatores tais como tempo, condições do solo e prática de cultivo, que influenciam a produção de maçãs em determinado ano. Assim, cada produtor em cada ano tem um valor para IP e são agrupados em sete categorias: $(0 ; 0,15],(0,15 ; 0,30]$, $(0,30 ; 0,45],(0,45 ; 0,60],(0,60 ; 0,80],(0,80 ; 1,00]$ e $(1,00 ; \infty)$. Para cada categoria e cada variedade tem-se o registro da produção total de maçãs e o número de árvores em cada idade. Isso sugere naturalmente um modelo de regressão linear em que a variável dependente é a produção total de maçãs e as variáveis regressoras são o número de árvores em cada idade, isto é,

$$
y_{i}=\sum_{j=2}^{11} \beta_{j} x_{i j}+\varepsilon_{i},
$$

onde $\varepsilon_{i} \sim \mathrm{N}\left(0, \sigma^{2}\right)$ e $x_{i j}$ é o número de árvores com idade $j$ referente ao $i$-ésimo produtor. Além disso, $\beta_{j}$ representa o número médio de caixas de maçãs produzidas pelas árvores de idade $j$ e como os produtores usualmente eliminam árvores não produtivas, é bastante 
razoável restringir os parâmetros do modelo à forma

$$
0 \leq \beta_{2} \leq \ldots \leq \beta_{11}
$$

a qual é chamada de restrições monótonas. Normalmente, essa suposição é aceita a priori pelos produtores e, consequentemente, deve ser incluída na análise dos dados usados no modelo de previsão. O problema então é determinar uma equação de regressão apropriada para esses dados e usá-la para prever a produção de um determinado produtor em cada categoria. Chen e Deely (1996) compararam a metodologia de mínimos quadrados com uma metodologia Bayesiana através de um modelo de regressão múltipla levando em conta as restrições nos parâmetros do modelo proposto.

Nesses dois exemplos tem-se respostas Gaussianas e independentes, no entanto, existem situações em que as respostas podem ser correlacionadas e a suposição de normalidade pode não ser adequada. Apenas para ilustrar, considere inicialmente o modelo descrito por Wolak (1989) para explicar a demanda de energia elétrica agregada:

$$
\begin{aligned}
\ln \left(Q_{t}\right) & =\alpha+\beta_{1} \ln \left(P E_{t}\right)+\beta_{2} \ln \left(P G_{t}\right)+\beta_{3} \ln \left(Y_{t}\right) \\
& +\beta_{4} D 1_{t}+\beta_{5} D 2_{t}+\beta_{6} D 3_{t}+\varepsilon_{t},
\end{aligned}
$$

onde $\varepsilon_{t}=\rho \varepsilon_{t-1}+\eta_{t}$ e $\eta_{t} \sim \mathrm{N}\left(0, \sigma^{2}\right)$. As variáveis $Q_{t}, P E_{t}, P G_{t}$ e $Y_{t}$ representam, respectivamente, a demanda de eletricidade agregada per capita para o setor residencial, o preço médio da eletricidade para o setor residencial, o preço do gás natural para o setor residencial e a renda per capita. $D 1_{t}, D 2_{t}$ e $D 3_{t}$ são variáveis binárias e foram incluídas porque os dados estão em bases trimestrais. $t$ representa o trimestre e os dados foram coletados desde o primeiro trimestre de 1961 até o quarto trimestre de 1983. É bastante razoável esperar que a demanda de eletricidade cresça quando diminui o preço da eletricidade e aumenta o preço do gás natural e a renda per capita, portanto, é de interesse testar inicialmente se o subvetor $\left(-\beta_{1}, \beta_{2}, \beta_{3}\right)^{T}$ é maior ou igual a zero. 
Num outro exemplo, discutido por Diggle et al. (1994, Seção 8.4), um grupo de 59 pacientes epilépticos foi aleatorizado de modo que um grupo (31 pacientes) recebesse uma droga anti-epiléptica enquanto um outro grupo (28 pacientes) recebesse placebo e foram acompanhados ao longo do tempo. A resposta observada foi o número de ataques epilépticos, inicialmente durante oito semanas antes do tratamento, e depois, durante quatro períodos de duas semanas cada um. Como trata-se de dados de contagem, é muito razoável pensar num modelo de Poisson para explicar o número médio de ataques epilépticos segundo os dois grupos e os períodos de estudo. Como os períodos não têm o mesmo comprimento (número de semanas) foi incluído o termo logaritmo natural do número de semanas como offset (vide, por exemplo, discussão em McCullagh e Nelder, 1989, p. 206). Assim, chamando de $y_{i j k}$ o número de ataques epilépticos sofridos pelo $k$ ésimo paciente do $i$-ésimo grupo no $j$-ésimo período, assumiu-se que $y_{i j k} \sim \operatorname{Poisson}\left(\mu_{j}\right)$, onde

$$
\begin{aligned}
& \ln \left(\mu_{10}\right)=\ln \left(t_{0}\right)+\alpha, \\
& \ln \left(\mu_{1 j}\right)=\ln \left(t_{j}\right)+\alpha+\beta, \\
& \ln \left(\mu_{20}\right)=\ln \left(t_{0}\right)+\alpha+\gamma \quad \mathrm{e} \\
& \ln \left(\mu_{2 j}\right)=\ln \left(t_{j}\right)+\alpha+\gamma+\beta+\delta,
\end{aligned}
$$

$i=1,2$ e $j=1,2,3,4, \alpha$ : nível base, $\beta$ : efeito de ensaio, $\gamma$ : efeito de grupo, $\delta$ : interação entre ensaio e grupo e $t_{j}$ : número de semanas referente ao $j$-ésimo período. Note que a razão entre o número médio de ataques epilépticos dos dois grupos é, antes do tratamento, dada por

$$
\frac{\mu_{20}}{\mu_{10}}=\exp (\gamma)
$$

e, depois do tratamento,

$$
\frac{\mu_{2 j}}{\mu_{1 j}}=\exp (\gamma+\delta)
$$

$j=1,2,3,4$. Parece ser muito razoável esperar que após o tratamento a razão entre as 
médias não aumente, isto é, que

$$
\frac{\mu_{2 j}}{\mu_{1 j}} \leq \frac{\mu_{20}}{\mu_{10}}, \forall j
$$

que é o mesmo que assumir $\delta \leq 0$. Assim, tem-se um modelo log-linear de Poisson com respostas correlacionadas e restrição em desigualdade num dos parâmetros.

Recentemente, Hadgu e Koch (1999) apresentaram um estudo em que 109 adultos voluntários com pré-existência de placa dentária foram aleatorizados em três grupos: controle (39 indivíduos), A (34 indivíduos) e B (36 indivíduos). Os adultos do grupo controle receberam um líquido padrão para ser usado para gargarejo após a escovação enquanto os adultos dos grupos A e B receberam líquidos diferentes desenvolvidos para reduzir o índice médio de placas dentárias. A variável resposta considerada foi o índice de placas dentárias. Os indivíduos foram observados em três momentos diferentes: antes da aplicação dos tratamentos, após 3 meses de aplicação e após 6 meses de aplicação. Maiores detalhes sobre o experimento são encontrados no próprio artigo de Hadgu e Koch (1999). O principal objetivo do estudo é verificar se os dois líquidos experimentais reduzem o índice médio de placas dentárias. Os pesquisadores verificaram que a variável de interesse, índice de placas dentárias, se aproxima mais de uma gama com coeficiente de variação constante do que de uma normal. Assim, denotando por $y_{i j k}$ o índice de placas dentárias do $k$-ésimo indivíduo do $i$-ésimo grupo no $j$-ésimo período, assumiu-se que $y_{i j k} \sim \operatorname{Gama}\left(\mu_{i j}, \phi\right)$. Como a ligação log apresenta os efeitos em forma multiplicativa, optou-se inicialmente por um modelo gama log linear em que

$$
\log \left(\mu_{i j}\right)=\alpha+\beta_{i}+\gamma_{j}
$$

$i=1,2,3$ e $j=1,2,3, \beta_{i}$ : efeito do $i$-ésimo tratamento e $\gamma_{j}$ : efeito do $j$-ésimo período, com $\beta_{1}=0$ (controle) e $\gamma_{1}=0$ (nível base). Parece, também neste caso, ser razoável esperar que o índice médio de placas dentárias não aumente com os tratamentos A e B 
em relação ao grupo controle. Isso implica em $\mu_{1 j} \geq \mu_{2 j}$ e $\mu_{1 j} \geq \mu_{3 j}, \forall j$, que leva às restrições $\beta_{2} \leq 0$ e $\beta_{3} \leq 0$. No nível base estamos assumindo que as médias são iguais. Portanto, tem-se nesse exemplo variável resposta gama, dados correlacionados e dois dos parâmetros restritos em desigualdades.

A teoria de estimação e testes em modelos de regressão em que os parâmetros são restritos na forma de desigualdades, foi introduzido por Bartholomew (1959a,b, 1961a,b) e por Kudô (1963) e durante três décadas ficou fortemente concentrada em modelos com resposta Gaussiana, embora tenham surgido nesse período vários trabalhos envolvendo a família exponencial de distribuições, porém, sem o uso de regressores. A partir da década de 90 vários trabalhos foram desenvolvidos para modelos não Gaussianos (vide, por exemplo, Piegorsch, 1990; McDonald e Diamond, 1990; Russek-Cohen e Simon, 1993; Silvapulle, 1994; Fahrmeir e Klinger, 1994; Paula e Sen, 1995; Paula e Rojas, 1997 e Paula, 1999), muitos dos quais envolvendo modelos lineares generalizados e apenas um trabalho (Park et al., 1998) usando equações de estimação generalizadas (EEGs), embora restrito ao caso de comparação de médias.

Neste trabalho propomos, inicialmente, unificar os principais artigos no assunto envolvendo modelos lineares com resposta Gaussiana. Fazemos isso no Capítulo 2. Em seguida, no Capítulo 3, discutimos as extensões desenvolvidas para os modelos lineares generalizados e apresentamos possíveis adaptações usando EEGs. É importante ressaltar, que no caso de EEGs, não se conhece totalmente a função de verossimilhança o que limita o desenvolvimento de testes de hipóteses. Estudamos, em particular, a aplicação do procedimento proposto por Kodde e Palm (1986) e resultados de Wolak (1991) a fim de desenvolvermos testes para hipóteses restritas em desigualdades usando EEGs. Vários estudos de simulação são desenvolvidos no Capítulo 4 com o intuito de investigarmos o comportamento dos procedimentos propostos. 
No Capítulo 5, estendemos uma proposta recente de Follmann (1996) para testar hipóteses restritas através da estatística $T^{2}$ de Hotteling para EEGs. Novamente, vários estudos de simulação são desenvolvidos a fim de compararmos o procedimento adaptado de Follmann para EEGs com o procedimento de Kodde e Palm. Finalmente, no Capítulo 6, resumimos os principais resultados obtidos neste trabalho. 


\section{Capítulo 2}

\section{Modelo Linear Geral}

\subsection{Introdução}

Os modelos lineares são largamente utilizados na estatística para descrever fenômenos da natureza e, em muitos casos, o objetivo é predizer ou descrever uma variável resposta $\boldsymbol{y}=\left(y_{1}, y_{2}, \ldots, y_{n}\right)^{T}$ em termos de outras variáveis, as quais são chamadas regressoras, covariáveis ou variáveis explicativas.

A análise de regressão é muito utilizada para descrever a relação entre a esperança da variável resposta e as covariáveis. O modelo de regressão linear assume a seguinte relação:

$$
y_{i}=\beta_{1} x_{1 i}+\beta_{2} x_{2 i}+\cdots+\beta_{p} x_{p i}+\varepsilon_{i}, \quad i=1,2, \ldots, n,
$$

onde $\boldsymbol{\beta}=\left(\beta_{1}, \beta_{2}, \ldots, \beta_{p}\right)^{T}$ é um vetor $(p \times 1)$ de parâmetros desconhecidos a ser estimado, $\varepsilon=\left(\varepsilon_{1}, \varepsilon_{2}, \ldots, \varepsilon_{n}\right)^{T}$ é um vetor de erros não observáveis e $x_{1 i}, x_{2 i}, \ldots, x_{p i}$ são os valores observados de $p$ variáveis explicativas. 
Usualmente $x_{1 i}=1$ para todo $i$, de modo que $\beta_{1}$ é o intercepto do modelo de regressão e supõe-se no caso mais simples que os $\varepsilon_{i}$ 's são variáveis aleatórias não correlacionadas com média zero e variância $\sigma^{2}, i=1,2, \ldots, n$. Isso implica que $\mu_{i}=\mathrm{E}\left(y_{i}\right)=\boldsymbol{x}_{i}^{T} \boldsymbol{\beta}$ e $\operatorname{Var}\left(y_{i}\right)=\sigma^{2}$, onde $\boldsymbol{x}_{i}^{T}=\left(x_{1 i}, x_{2 i}, \ldots, x_{p i}\right)$. Esse modelo pode ser escrito na forma matricial

$$
y=X \beta+\varepsilon
$$

onde $\boldsymbol{X}$ é uma matriz $(n \times p)$ cujas linhas são formadas por $\boldsymbol{x}_{i}^{T}, i=1,2, \ldots, n$.

Neste capítulo vamos apresentar o modelo de regressão normal linear geral, a estimação dos parâmetros e alguns testes estatísticos tradicionais para hipóteses restritas na forma de desigualdades lineares.

\subsection{Modelo de regressão normal linear}

Considere o modelo de regressão linear (2.1.2). O modelo de regressão normal linear geral é composto por (2.1.2) com a suposição de que $\varepsilon \sim \mathrm{N}_{n}(\mathbf{0}, \boldsymbol{\Sigma})$, onde $\boldsymbol{\Sigma}$ é uma matriz $(n \times n)$ positiva definida e 0 é o vetor nulo $n \times 1$, isto é

$$
\boldsymbol{y}=\boldsymbol{X} \boldsymbol{\beta}+\varepsilon, \quad \varepsilon \sim \mathrm{N}_{n}(\mathbf{0}, \boldsymbol{\Sigma})
$$

Portanto, a distribuição conjunta do vetor $y$ é normal multivariada com média e variância dadas, respectivamente, por $\mathrm{E}(\boldsymbol{y})=\boldsymbol{X} \boldsymbol{\beta}$ e $\operatorname{Var}(\boldsymbol{y})=\boldsymbol{\Sigma}$. Essa classe de modelos lineares inclui como casos especiais a análise de variância, a análise de regressão simples e múltipla e a análise de covariância.

Quando não há restrições sobre o vetor de parâmetros $\beta$, o estimador de mínimos 
quadrados $\hat{\boldsymbol{\beta}}$ é obtido como solução do sistema de equações normais

$$
\boldsymbol{X}^{T} \boldsymbol{\Sigma}^{-1} \boldsymbol{y}-\boldsymbol{X}^{T} \boldsymbol{\Sigma}^{-1} \boldsymbol{X} \hat{\boldsymbol{\beta}}=\mathbf{0} .
$$

Assumindo que a matriz $\boldsymbol{X}$ é de posto completo, então $\boldsymbol{X}^{T} \boldsymbol{\Sigma}^{-1} \boldsymbol{X}$ é inversível e o estimador de mínimos quadrados de $\boldsymbol{\beta}$ fica dado por

$$
\hat{\boldsymbol{\beta}}=\left(\boldsymbol{X}^{T} \Sigma^{-1} \boldsymbol{X}\right)^{-1} \boldsymbol{X}^{T} \Sigma^{-1} \boldsymbol{y}
$$

O estimador de mínimos quadrados $\hat{\boldsymbol{\beta}}$ satisfaz importantes propriedades estatísticas. É um estimador não tendencioso para o vetor de parâmetros $\boldsymbol{\beta}$, isto é,

$$
\mathrm{E}(\hat{\boldsymbol{\beta}})=\left(\boldsymbol{X}^{T} \boldsymbol{\Sigma}^{-1} \boldsymbol{X}\right)^{-1} \boldsymbol{X}^{T} \boldsymbol{\Sigma}^{-1} \boldsymbol{X} \boldsymbol{\beta}=\boldsymbol{\beta}
$$

e sua matriz de variância é dada por

$$
\operatorname{Var}(\hat{\boldsymbol{\beta}})=\left(\boldsymbol{X}^{T} \boldsymbol{\Sigma}^{-1} \boldsymbol{X}\right)^{-1} \boldsymbol{X}^{T} \boldsymbol{\Sigma}^{-1} \boldsymbol{\Sigma} \boldsymbol{\Sigma}^{-1} \boldsymbol{X}\left(\boldsymbol{X}^{T} \boldsymbol{\Sigma}^{-1} \boldsymbol{X}\right)^{-1}=\left(\boldsymbol{X}^{T} \boldsymbol{\Sigma}^{-1} \boldsymbol{X}\right)^{-1}
$$

Além disso, para qualquer vetor $\boldsymbol{v}(p \times 1)$, se $\boldsymbol{\theta}=\boldsymbol{v}^{T} \boldsymbol{\beta}$ então, o teorema de GaussMarkov garante que $\hat{\boldsymbol{\theta}}=\boldsymbol{v}^{T} \hat{\boldsymbol{\beta}}$ tem variância mínima na classe de todos os estimadores não viesados para $\boldsymbol{\theta}$ que são combinações lineares de $\boldsymbol{y}$.

Quando a matriz $\boldsymbol{X}$ não é de posto completo, então o sistema de equações normais (2.2.2) não tem solução única e neste caso a matriz inversa $\left(\boldsymbol{X}^{T} \boldsymbol{\Sigma}^{-1} \boldsymbol{X}\right)^{-1}$ em (2.2.3) é substituída por uma inversa generalizada $\left(\boldsymbol{X}^{T} \boldsymbol{\Sigma}^{-1} \boldsymbol{X}\right)^{-}$e obtém-se resultados análogos ao caso em que o posto de $\boldsymbol{X}$ é completo. Para mais detalhes sobre o modelo de regressão normal linear geral, veja por exemplo, Rao (1973).

\subsection{Estimação com parâmetros restritos}

Considere agora o modelo de regressão normal linear (2.2.1) com $\Sigma$ conhecida e suponha que o vetor de parâmetros $\boldsymbol{\beta}$ está sujeito a restrições do tipo $\boldsymbol{R} \boldsymbol{\beta} \geq \boldsymbol{r}$, onde $\boldsymbol{R}$ é uma 
matriz $(k \times p)$ conhecida, de posto completo e $r$ é um vetor $(k \times 1)$ de constantes conhecidas. Nesse caso, o estimador de mínimos quadrados de $\boldsymbol{\beta}$ será denotado por $\tilde{\boldsymbol{\beta}}$ e é a solução do seguinte Problema de Programação Quadrática (PPQ):

$$
\min _{\boldsymbol{\beta}}\left\{(\boldsymbol{y}-\boldsymbol{X} \boldsymbol{\beta})^{T} \boldsymbol{\Sigma}^{-1}(\boldsymbol{y}-\boldsymbol{X} \boldsymbol{\beta})\right\} \quad \text { sujeito a } \quad R \boldsymbol{\beta} \geq \boldsymbol{r}
$$

O vetor de multiplicadores de Kuhn-Tucker associado com a restrição $\boldsymbol{R} \boldsymbol{\beta} \geq \boldsymbol{r}$ será denotado por $\tilde{\lambda}$. Existem vários algoritmos para resolver esse problema, veja por exemplo, Luenberger (1969), Avriel (1976) e Bazaraa et al. (1993). Quando não há restrições sobre o vetor de parâmetros $\boldsymbol{\beta}$, derivando-se a função objetiva em (2.3.1) com relação a $\boldsymbol{\beta}$ e igualando a zero, obtém-se $\hat{\boldsymbol{\beta}}$, o estimador de mínimos quadrados não restrito de $\boldsymbol{\beta}$ dado em (2.2.3), o qual segue uma distribuição normal multivariada com média $\boldsymbol{\beta}$ e matriz de covariância $\left(\boldsymbol{X}^{T} \Sigma^{-1} \boldsymbol{X}\right)^{-1}$.

Um algoritmo simples para resolver o problema (2.3.1) é uma consequência dos resultados de Theil e Van de Panne (1960). Observe que quando $\boldsymbol{R} \hat{\boldsymbol{\beta}} \geq \boldsymbol{r}$, então $\tilde{\boldsymbol{\beta}}$ coincide com $\hat{\boldsymbol{\beta}}$. Se esse não é o caso, eles provam que se $m \leq k$ componentes do vetor $\boldsymbol{R} \hat{\boldsymbol{\beta}}$ não satisfazem as restrições de desigualdades lineares, pelo menos uma das desigualdades violadas é satisfeita na forma de igualdade linear, isto é, se ocorrem $m$ violações, deveremos resolver $2^{m}-1$ problemas de otimização restritos por igualdades lineares para obter $\tilde{\boldsymbol{\beta}}$. Para ilustrar esse procedimento (veja, por exemplo, Paula, 1997), suponha a ocorrência de $m=2$ violações, $\mathbf{R}_{1}^{T} \hat{\boldsymbol{\beta}}<r_{1}$ e $\mathbf{R}_{2}^{T} \hat{\boldsymbol{\beta}}<r_{2}$, onde $\mathbf{R}_{i}^{T}$ e $r_{i}$ correspondem, respectivamente, à $i$-ésima linha da matriz $\boldsymbol{R}$ e ao $i$-ésimo elemento do vetor $\boldsymbol{r}$. Para determinar $\tilde{\boldsymbol{\beta}}$, deveremos resolver os três seguintes problemas de otimização:
1) $\min _{\boldsymbol{\beta}}\left\{(\boldsymbol{y}-\boldsymbol{X} \boldsymbol{\beta})^{T} \boldsymbol{\Sigma}^{-1}(\boldsymbol{y}-\boldsymbol{X} \boldsymbol{\beta})\right\}$ sujeito a $\boldsymbol{R}_{1}^{T} \boldsymbol{\beta}=r_{1}$;
2) $\min _{\boldsymbol{\beta}}\left\{(\boldsymbol{y}-\boldsymbol{X} \boldsymbol{\beta})^{T} \boldsymbol{\Sigma}^{-1}(\boldsymbol{y}-\boldsymbol{X} \boldsymbol{\beta})\right\}$ sujeito a $\boldsymbol{R}_{2}^{T} \boldsymbol{\beta}=r_{2}$ e
3) $\min _{\boldsymbol{\beta}}\left\{(\boldsymbol{y}-\boldsymbol{X} \boldsymbol{\beta})^{T} \boldsymbol{\Sigma}^{-1}(\boldsymbol{y}-\boldsymbol{X} \boldsymbol{\beta})\right\} \quad$ sujeito a $\boldsymbol{R}_{1}^{T} \boldsymbol{\beta}=r_{1}, \boldsymbol{R}_{2}^{T} \boldsymbol{\beta}=r_{2}$. 
Das três soluções encontradas, aquela que não apresentar violação e levar ao menor valor da função objetiva será a estimativa procurada. A principal vantagem desse procedimento é que o estimador de mínimos quadrados restrito por igualdades lineares é sempre expresso em forma fechada, evitando o uso de procedimentos de Programação Quadrática.

Vamos denotar por $\overline{\boldsymbol{\beta}}$, o estimador de mínimos quadrados de $\boldsymbol{\beta}$ sujeito à restrição $\boldsymbol{R} \boldsymbol{\beta}=\boldsymbol{r}$, ou seja, $\overline{\boldsymbol{\beta}}$ é a solução de

$$
\min _{\boldsymbol{\beta}}\left\{(\boldsymbol{y}-\boldsymbol{X} \boldsymbol{\beta})^{T} \boldsymbol{\Sigma}^{-1}(\boldsymbol{y}-\boldsymbol{X} \boldsymbol{\beta})\right\} \quad \text { sujeito a } \quad \boldsymbol{R} \boldsymbol{\beta}=\boldsymbol{r}
$$

O vetor de multiplicadores de Lagrange associado à restrição $\boldsymbol{R} \boldsymbol{\beta}=\boldsymbol{r}$ será denotado por $\bar{\lambda}$ e por convenção, será associado ao estimador não restrito o vetor de multiplicadores $\hat{\lambda}$, o qual é identicamente nulo. Com isso, esses estimadores satisfazem

$$
\frac{\partial}{\partial \boldsymbol{\beta}}\left\{(\boldsymbol{y}-\boldsymbol{X} \boldsymbol{\beta})^{T} \boldsymbol{\Sigma}^{-1}(\boldsymbol{y}-\boldsymbol{X} \boldsymbol{\beta})-\lambda^{T}(\boldsymbol{R} \boldsymbol{\beta}-\boldsymbol{r})\right\}=0
$$

e essa condição implica em

$$
\begin{gathered}
\frac{\partial}{\partial \boldsymbol{\beta}}\left\{\boldsymbol{y}^{T} \boldsymbol{\Sigma}^{-1} \boldsymbol{y}-2 \boldsymbol{y}^{T} \boldsymbol{\Sigma}^{-1} \boldsymbol{X} \boldsymbol{\beta}+\boldsymbol{\beta}^{T} \boldsymbol{X}^{T} \boldsymbol{\Sigma}^{-1} \boldsymbol{X} \boldsymbol{\beta}-\boldsymbol{\lambda}^{T}(\boldsymbol{R} \boldsymbol{\beta}-\boldsymbol{r})\right\}=\mathbf{0} \\
-2 \boldsymbol{X}^{T} \boldsymbol{\Sigma}^{-1} \boldsymbol{y}+2 \boldsymbol{X}^{T} \boldsymbol{\Sigma}^{-1} \boldsymbol{X} \overline{\boldsymbol{\beta}}-\boldsymbol{R}^{T} \bar{\lambda}=\mathbf{0}
\end{gathered}
$$

Portanto,

$$
\overline{\boldsymbol{\beta}}=\left(\boldsymbol{X}^{T} \boldsymbol{\Sigma}^{-1} \boldsymbol{X}\right)^{-1} \boldsymbol{X}^{T} \boldsymbol{\Sigma}^{-1} \boldsymbol{y}+\frac{1}{2}\left(\boldsymbol{X}^{T} \boldsymbol{\Sigma}^{-1} \boldsymbol{X}\right)^{-1} \boldsymbol{R}^{T} \bar{\lambda}
$$

e usando (2.2.3) temos que

$$
\overline{\boldsymbol{\beta}}=\hat{\boldsymbol{\beta}}+\frac{1}{2}\left(\boldsymbol{X}^{T} \boldsymbol{\Sigma}^{-1} \boldsymbol{X}\right)^{-1} \boldsymbol{R}^{T} \bar{\lambda}
$$

Da mesma forma temos que

$$
\tilde{\boldsymbol{\beta}}=\hat{\boldsymbol{\beta}}+\frac{1}{2}\left(\boldsymbol{X}^{T} \boldsymbol{\Sigma}^{-1} \boldsymbol{X}\right)^{-1} \boldsymbol{R}^{T} \tilde{\lambda}
$$


e obtém-se a relação

$$
\tilde{\boldsymbol{\beta}}-\overline{\boldsymbol{\beta}}=\frac{1}{2}\left(\boldsymbol{X}^{T} \Sigma^{-1} \boldsymbol{X}\right)^{-1} \boldsymbol{R}^{T}(\tilde{\lambda}-\bar{\lambda}) .
$$

Essa relação entre os três estimadores será bastante útil nas seções seguintes.

Como devemos ter $\boldsymbol{R} \overline{\boldsymbol{\beta}}=\boldsymbol{r}$ em (2.3.2), então

$$
\begin{gathered}
\boldsymbol{R} \hat{\boldsymbol{\beta}}+\frac{1}{2} \boldsymbol{R}\left(\boldsymbol{X}^{T} \Sigma^{-1} \boldsymbol{X}\right)^{-1} \boldsymbol{R}^{T} \bar{\lambda}=r \\
2(\boldsymbol{r}-\boldsymbol{R} \hat{\boldsymbol{\beta}})=\boldsymbol{R}\left(\boldsymbol{X}^{T} \boldsymbol{\Sigma}^{-1} \boldsymbol{X}\right)^{-1} \boldsymbol{R}^{T} \bar{\lambda} \mathrm{e} \\
\bar{\lambda}=2\left\{\boldsymbol{R}\left(\boldsymbol{X}^{T} \Sigma^{-1} \boldsymbol{X}\right)^{-1} \boldsymbol{R}^{T}\right\}^{-1}(\boldsymbol{r}-\boldsymbol{R} \hat{\boldsymbol{\beta}}) .
\end{gathered}
$$

Portanto, o estimador de mínimos quadrados de $\boldsymbol{\beta}$ sujeito à restrição $\boldsymbol{R} \boldsymbol{\beta}=\boldsymbol{r}$ é dado por

$$
\overline{\boldsymbol{\beta}}=\hat{\boldsymbol{\beta}}+\left(\boldsymbol{X}^{T} \boldsymbol{\Sigma}^{-1} \boldsymbol{X}\right)^{-1} \boldsymbol{R}^{T}\left\{\boldsymbol{R}\left(\boldsymbol{X}^{T} \boldsymbol{\Sigma}^{-1} \boldsymbol{X}\right)^{-1} \boldsymbol{R}^{T}\right\}^{-1}(\boldsymbol{r}-\boldsymbol{R} \hat{\boldsymbol{\beta}}) .
$$

Assim, $\overline{\boldsymbol{\beta}}$ é normalmente distribuído com média e variância dadas por

$$
\begin{aligned}
\mathrm{E}(\overline{\boldsymbol{\beta}}) & =\boldsymbol{\beta}+\left(\boldsymbol{X}^{T} \boldsymbol{\Sigma}^{-1} \boldsymbol{X}\right)^{-1} \boldsymbol{R}^{T}\left\{\boldsymbol{R}\left(\boldsymbol{X}^{T} \boldsymbol{\Sigma}^{-1} \boldsymbol{X}\right)^{-1} \boldsymbol{R}^{T}\right\}^{-1}(\boldsymbol{r}-\boldsymbol{R} \boldsymbol{\beta}) \text { e } \\
\operatorname{Var}(\overline{\boldsymbol{\beta}}) & =\left(\boldsymbol{X}^{T} \boldsymbol{\Sigma}^{-1} \boldsymbol{X}\right)^{-1}+ \\
& +\left(\boldsymbol{X}^{T} \boldsymbol{\Sigma}^{-1} \boldsymbol{X}\right)^{-1} \boldsymbol{R}^{T}\left\{\boldsymbol{R}\left(\boldsymbol{X}^{T} \boldsymbol{\Sigma}^{-1} \boldsymbol{X}\right)^{-1} \boldsymbol{R}\right\}^{-1} \boldsymbol{R}\left(\boldsymbol{X}^{T} \boldsymbol{\Sigma}^{-1} \boldsymbol{X}\right)^{-1} \\
& =\left(\boldsymbol{X}^{T} \boldsymbol{\Sigma}^{-1} \boldsymbol{X}\right)^{-1}\left\{\boldsymbol{I}_{p}+\boldsymbol{R}^{T}\left\{\boldsymbol{R}\left(\boldsymbol{X}^{T} \boldsymbol{\Sigma}^{-1} \boldsymbol{X}\right)^{-1} \boldsymbol{R}^{T}\right\}^{-1} \boldsymbol{R}\left(\boldsymbol{X}^{T} \boldsymbol{\Sigma}^{-1} \boldsymbol{X}\right)^{-1}\right\}
\end{aligned}
$$

onde $\boldsymbol{I}_{p}$ é a matriz identidade de ordem $p$.

A distribuição exata de $\tilde{\boldsymbol{\beta}}$ é bastante complicada de ser obtida. Há uma discussão em Judge e Yancey (1986) sobre a estimação e propriedades de $\tilde{\boldsymbol{\beta}}$. Liew (1976) mostra que, quando a restrição $\boldsymbol{R} \boldsymbol{\beta}>\boldsymbol{r}$ é verdadeira, a distribuição assintótica de $\tilde{\boldsymbol{\beta}}$ reduzse à distribuição de $\hat{\boldsymbol{\beta}}$ e portanto $\tilde{\boldsymbol{\beta}}$ é também normal multivariada. No entanto, os problemas ocorrem quando o verdadeiro vetor de parâmetros pode pertencer à fronteira 
$\boldsymbol{R} \boldsymbol{\beta}=\boldsymbol{r}$ do espaço paramétrico. A título de ilustração, vamos determinar a distribuição de $\tilde{\boldsymbol{\beta}}$ no seguinte modelo de regressão linear (veja, por exemplo, Paula e Bolfarine, 1997):

$$
y_{i}=\beta x_{i}+\varepsilon_{i}, \quad \text { sujeito a } \quad \beta \geq 1 \text {, }
$$

onde $\varepsilon_{i} \sim N\left(0, \sigma^{2}\right), \quad i=1,2, \ldots, n$, são independentes, $\beta$ é um parâmetro escalar e $\left(x_{i}, y_{i}\right)$ são observações de um experimento do tipo pré-teste pós-teste. O estimador não restrito de mínimos quadrados de $\beta$ é dado por

$$
\hat{\beta}=\frac{\sum_{i=1}^{n} x_{i} y_{i}}{\sum_{i=1}^{n} x_{i}^{2}},
$$

e portanto $\hat{\beta}$ é normalmente distribuído com média $\beta$ e variância $v=\sigma^{2} / \sum_{i=1}^{n} x_{i}^{2}$.

O estimador de mínimos quadrados restrito é dado por

$$
\tilde{\beta}=\left\{\begin{array}{l}
\hat{\beta} \text { se } \hat{\beta}>1 \\
1 \text { se } \hat{\beta} \leq 1
\end{array},\right.
$$

e portanto a distribuição de $\tilde{\beta}$ é uma normal truncada. Seja $\beta_{0}$ o verdadeiro valor do parâmetro $\beta$, então temos que

$$
P(\tilde{\beta}=1)=P(\hat{\beta} \leq 1)=\Phi\left\{\left(1-\beta_{0}\right) / \sqrt{v}\right\}
$$

onde $\Phi(\cdot)$ representa a função de distribuição acumulada da normal padrão. Para qualquer $c>1$, temos que

$$
P(\tilde{\beta} \leq c)=P(\hat{\beta} \leq c)=\Phi\left\{\left(c-\beta_{0}\right) / \sqrt{v}\right\}
$$

e portanto a função densidade de probabilidade de $\tilde{\beta}$ pode ser expressa na seguinte forma:

$$
f_{\tilde{\beta}}(c)=\Phi\left(\frac{1-\beta_{0}}{\sqrt{v}}\right) u_{(1)}+\phi\left(\frac{c-\beta_{0}}{\sqrt{v}}\right) \mathbf{I}_{[1, \infty)}(c),
$$

onde $u_{(1)}$ denota a distribuição degenerada em $1, \mathbf{I}_{\{\cdot\}}(\cdot)$ representa a função indicadora e $\phi(\cdot)$ é a função densidade de probabilidade da normal padrão. 
Note que o estimador restrito $\tilde{\beta}$ pode ser escrito na forma

$$
\begin{aligned}
\tilde{\beta} & =\hat{\beta} \mathbf{I}_{(1, \infty)}(\hat{\beta})+\mathbf{I}_{(-\infty, 1]}(\hat{\beta}) \\
& =\hat{\beta}+(1-\beta) \mathbf{I}_{(-\infty, 1-\beta)}(\hat{\beta}-\beta)-(\hat{\beta}-\beta) \mathbf{I}_{(-\infty, 1-\beta)}(\hat{\beta}-\beta) .
\end{aligned}
$$

Considerando $\sigma^{2}$ conhecido, podemos assumir, sem perda de generalidade, que $v=1$, e usando alguns resultados dados em Judge e Yancey (1986) temos para $\beta>1$ que

$$
\begin{aligned}
\mathrm{E}\left\{\mathbf{I}_{(-\infty, 1-\beta)}(\hat{\beta}-\beta)\right\} & =\frac{1}{2} P\left\{\chi_{1}^{2} \geq(1-\beta)^{2}\right\} \\
\mathrm{E}\left\{(\hat{\beta}-\beta) \mathbf{I}_{(-\infty, 1-\beta)}(\hat{\beta}-\beta)\right\} & =-\frac{1}{\sqrt{2 \pi}} P\left\{\chi_{2}^{2} \geq(1-\beta)^{2}\right\} \mathrm{e} \\
\mathrm{E}\left\{(\hat{\beta}-\beta)^{2} \mathbf{I}_{(-\infty, 1-\beta)}(\hat{\beta}-\beta)\right\} & =\frac{1}{2} P\left\{\chi_{3}^{2} \geq(1-\beta)^{2}\right\} .
\end{aligned}
$$

Assim, após algumas manipulações algébricas o viés e o erro quadrático médio de $\tilde{\beta}$ ficam, respectivamente, dados por

$$
\begin{gathered}
\mathrm{B}(\tilde{\beta})=\mathrm{E}\{\tilde{\beta}-\beta\}=-\frac{(1-\beta)}{2} P\left\{\chi_{1}^{2} \geq(1-\beta)^{2}\right\}+\frac{1}{\sqrt{2 \pi}} P\left\{\chi_{2}^{2} \geq(1-\beta)^{2}\right\} \text { e } \\
\operatorname{EQM}(\tilde{\beta})=\mathrm{E}\{\tilde{\beta}-\beta\}^{2}=1+\frac{(1-\beta)^{2}}{2} P\left\{\chi_{1}^{2} \geq(1-\beta)^{2}\right\}-\frac{1}{2} P\left\{\chi_{3}^{2} \geq(1-\beta)^{2}\right\} .
\end{gathered}
$$

Note que $\mathrm{B}(\tilde{3}) \rightarrow 0$ conforme $\beta \rightarrow \infty$ e $\mathrm{B}(\tilde{\beta}) \rightarrow 1 / \sqrt{2 \pi}$ conforme $\beta \rightarrow 1$. Além disso, $\operatorname{EQM}(\tilde{\beta}) \rightarrow 1 / 2$ conforme $\beta \rightarrow 1$ e $\operatorname{EQM}(\tilde{\beta}) \rightarrow 1$ conforme $\beta \rightarrow \infty$. Portanto, $\tilde{\beta}$ apresenta um erro quadrático médio menor do que o erro quadrático médio de $\hat{\beta}$, com viés indo para zero conforme $\beta \rightarrow \infty$. Para ter os resultados na escala original deve-se substituir $\beta$ por $\beta / \sqrt{v}$.

Em Gouriéroux e Monfort (1995, Capítulo 21) há uma discussão interessante sobre a determinação do estimador de mínimos quadrados restrito num modelo específico de regressão normal linear. 


\subsection{Estatísticas equivalentes}

Considere o modelo de regressão normal linear (2.2.1) e suponha novamente $\boldsymbol{\Sigma}$ conhecida e positiva definida. O interesse é determinar as estatísticas do teste para as seguintes hipóteses:

$$
\begin{array}{ll}
\text { (Caso I) } & H_{0}: \boldsymbol{R} \boldsymbol{\beta}=\boldsymbol{r} \text { versus } H_{1}: \boldsymbol{R} \boldsymbol{\beta} \geq \boldsymbol{r}, \\
(\text { Caso II) } & H_{1}: \boldsymbol{R} \boldsymbol{\beta} \geq \boldsymbol{r} \text { versus } H_{2}-H_{1},
\end{array}
$$

onde $H_{2}: \boldsymbol{\beta} \in \mathbb{R}^{p}$, com pelo menos uma desigualdade estrita em $H_{1}$ no caso I de modo que $H_{0} \subset H_{1} \subset H_{2}$.

\section{Caso I}

A estatística da razão de verossimilhança será denotada por $\xi_{r v}$ e para testar as hipóteses (2.4.1) é definida por

$$
\xi_{r v}=-2 \log \left\{\frac{\max _{\boldsymbol{\beta} \in H_{0}} L(\boldsymbol{\beta} \mid \boldsymbol{\Sigma})}{\max _{\boldsymbol{\beta} \in H_{\mathbf{1}}} L(\boldsymbol{\beta} \mid \boldsymbol{\Sigma})}\right\}=2\{\log \tilde{\mathcal{L}}-\log \overline{\mathcal{L}}\},
$$

onde $\overline{\mathcal{L}}$ e $\tilde{\mathcal{L}}$ representam o máximo da função de verossimilhança sob $H_{0}$ e $H_{1}$, respectivamente. Como $\boldsymbol{\Sigma}$ é conhecida, $\tilde{\boldsymbol{\beta}}$ e $\overline{\boldsymbol{\beta}}$, definidos na Seção 2.3 , são os estimadores de máxima verossimilhança de $\boldsymbol{\beta}$ sob $H_{1}$ e $H_{0}$, respectivamente. Isso implica em

$$
\xi_{r v}=(\boldsymbol{y}-\boldsymbol{X} \tilde{\boldsymbol{\beta}})^{T} \boldsymbol{\Sigma}^{-1}(\boldsymbol{y}-\boldsymbol{X} \tilde{\boldsymbol{\beta}})-(\boldsymbol{y}-\boldsymbol{X} \overline{\boldsymbol{\beta}})^{T} \boldsymbol{\Sigma}^{-1}(\boldsymbol{y}-\boldsymbol{X} \overline{\boldsymbol{\beta}}) .
$$

Podemos observar que a estatística da razão de verossimilhança também é o valor ótimo do seguinte PPQ:

$$
\left\{\begin{array}{l}
\min _{\boldsymbol{\beta}}\left\{(\boldsymbol{y}-\boldsymbol{X} \boldsymbol{\beta})^{T} \boldsymbol{\Sigma}^{-1}(\boldsymbol{y}-\boldsymbol{X} \boldsymbol{\beta})-(\boldsymbol{y}-\boldsymbol{X} \overline{\boldsymbol{\beta}})^{T} \boldsymbol{\Sigma}^{-1}(\boldsymbol{y}-\boldsymbol{X} \overline{\boldsymbol{\beta}})\right\} \\
\text { sujeito a } \boldsymbol{R} \boldsymbol{\beta} \geq \boldsymbol{r},
\end{array}\right.
$$


que é equivalente a

$$
\left\{\begin{array}{l}
\min _{\boldsymbol{\beta}}\left\{\boldsymbol{\beta}^{T}\left(\boldsymbol{X}^{T} \boldsymbol{\Sigma}^{-1} \boldsymbol{X}\right) \boldsymbol{\beta}-2 \boldsymbol{y}^{T} \boldsymbol{\Sigma}^{-1} \boldsymbol{X} \boldsymbol{\beta}+2 \boldsymbol{y}^{T} \boldsymbol{\Sigma}^{-1} \boldsymbol{X} \overline{\boldsymbol{\beta}}-\right. \\
\left.-\overline{\boldsymbol{\beta}}^{T}\left(\boldsymbol{X}^{T} \boldsymbol{\Sigma}^{-1} \boldsymbol{X}\right) \overline{\boldsymbol{\beta}}\right\} \text { sujeito a } \boldsymbol{R} \boldsymbol{\beta} \geq \boldsymbol{r} .
\end{array}\right.
$$

Esse PPQ pode ser colocado na forma padrão primal

$$
\min _{\boldsymbol{x}}\left\{\boldsymbol{a}+\boldsymbol{c}^{T} \boldsymbol{x}+\frac{1}{2} \boldsymbol{x}^{T} \boldsymbol{Q} \boldsymbol{x}\right\} \text { sujeito a } \boldsymbol{A x} \geq \boldsymbol{b},
$$

cujo dual é dado por

$$
\max _{\lambda}\left\{\lambda^{T}\left(b+\boldsymbol{A} Q^{-1} c\right)-\frac{1}{2} \lambda^{T} \boldsymbol{A} Q^{-1} A^{T} \lambda-\frac{1}{2} c^{T} Q^{-1} c+a\right\} \text { sujeito a } \lambda \geq 0
$$

Para detalhes sobre dualidade de programação quadrática veja Luenberger (1969), Avriel (1976) ou Bazaraa et al. (1993).

Usando o fato de que $\hat{\boldsymbol{\beta}}=\left(\boldsymbol{X}^{T} \boldsymbol{\Sigma}^{-1} \boldsymbol{X}\right)^{-1} \boldsymbol{X}^{T} \boldsymbol{\Sigma}^{-1} \boldsymbol{y}$ e a relação (2.3.3), o PPQ (2.4.5) é equivalente a

$$
\left\{\begin{array}{l}
\min _{\boldsymbol{\beta}}\left\{\boldsymbol{\beta}^{T}\left(\boldsymbol{X}^{T} \boldsymbol{\Sigma}^{-1} \boldsymbol{X}\right) \boldsymbol{\beta}-2 \boldsymbol{y}^{T} \boldsymbol{\Sigma}^{-1} \boldsymbol{X} \boldsymbol{\beta}+\right. \\
\left.+\boldsymbol{y}^{T} \boldsymbol{\Sigma}^{-1} \boldsymbol{X}\left(\boldsymbol{X}^{T} \boldsymbol{\Sigma}^{-1} \boldsymbol{X}\right)^{-1} \boldsymbol{X}^{T} \boldsymbol{\Sigma}^{-1} \boldsymbol{y}-\frac{1}{4} \overline{\boldsymbol{\lambda}}^{T} \boldsymbol{R}\left(\boldsymbol{X}^{T} \boldsymbol{\Sigma}^{-1} \boldsymbol{X}\right)^{-1} \boldsymbol{R}^{T} \bar{\lambda}\right\} \\
\text { sujeito a } \boldsymbol{R} \boldsymbol{\beta} \geq \boldsymbol{r}
\end{array}\right.
$$

Seguindo (2.4.6) e (2.4.7), o dual do PPQ (2.4.8) é dado por

$$
\left\{\begin{array}{c}
\max _{\lambda}\left\{\boldsymbol{\lambda}^{T}(\boldsymbol{r}-\boldsymbol{R} \hat{\boldsymbol{\beta}})-\frac{1}{4} \boldsymbol{\lambda}^{T} \boldsymbol{R}\left(\boldsymbol{X}^{T} \boldsymbol{\Sigma}^{-1} \boldsymbol{X}\right)^{-1} \boldsymbol{R}^{T} \boldsymbol{\lambda}-\right. \\
\left.-\frac{1}{4} \bar{\lambda}^{T} \boldsymbol{R}\left(\boldsymbol{X}^{T} \boldsymbol{\Sigma}^{-1} \boldsymbol{X}\right)^{-1} \boldsymbol{R}^{T} \bar{\lambda}\right\} \text { sujeito a } \lambda \geq 0 .
\end{array}\right.
$$

Mas,

$$
\lambda^{T}(\boldsymbol{r}-\boldsymbol{R} \hat{\boldsymbol{\beta}})=\frac{1}{2} \lambda^{T} \boldsymbol{R}\left(\boldsymbol{X}^{T} \boldsymbol{\Sigma}^{-1} \boldsymbol{X}\right)^{-1} \boldsymbol{R}^{T} \bar{\lambda}
$$

e portanto o dual do PPQ (2.4.8) torna-se

$$
\max _{\boldsymbol{\lambda}}\left\{-\frac{1}{4}(\boldsymbol{\lambda}-\overline{\boldsymbol{\lambda}})^{T} \boldsymbol{R}\left(\boldsymbol{X} \boldsymbol{\Sigma}^{-1} \boldsymbol{X}\right)^{-1} \boldsymbol{R}^{T}(\boldsymbol{\lambda}-\bar{\lambda})\right\} \text { sujeito a } \lambda \geq \mathbf{0}
$$


ou equivalentemente

$$
\min _{\boldsymbol{\lambda}}\left\{\frac{1}{4}(\boldsymbol{\lambda}-\bar{\lambda})^{T} \boldsymbol{R}\left(\boldsymbol{X} \boldsymbol{\Sigma}^{-1} \boldsymbol{X}\right)^{-1} \boldsymbol{R}^{T}(\boldsymbol{\lambda}-\bar{\lambda})\right\} \text { sujeito a } \lambda \geq 0 .
$$

A estatística de Kuhn-Tucker será denotada por $\xi_{k t}$ e é definida como sendo o valor ótimo da função objetiva em (2.4.10), o qual é atingido em $\tilde{\lambda}$. Portanto, temos que

$$
\xi_{k t}=\frac{1}{4}(\tilde{\boldsymbol{\lambda}}-\bar{\lambda})^{T} \boldsymbol{R}\left(\boldsymbol{X} \Sigma^{-1} \boldsymbol{X}\right)^{-1} \boldsymbol{R}^{T}(\tilde{\boldsymbol{\lambda}}-\bar{\lambda}) .
$$

Da teoria de Programação Quadrática, sabe-se que, sob certas condições de regularidade, o valor ótimo da função objetiva do problema primal é igual ao valor ótimo da função objetiva do problema dual, avaliados no ponto ótimo $(\tilde{\boldsymbol{\beta}}, \tilde{\boldsymbol{\lambda}})$. As condições de regularidade nesse caso estão satisfeitas pois, por suposição, $\Sigma$ é conhecida e positiva definida e portanto $\left(\boldsymbol{X}^{T} \boldsymbol{\Sigma}^{-1} \boldsymbol{X}\right)$ é não singular. Logo, temos a igualdade $\xi_{r v}=\xi_{k t}$.

A estatística de IVald será denotada por $\xi_{w}$ e para testar $H_{0}$ contra $H_{1}$ é dada por

$$
\begin{aligned}
\xi_{w} & =(\boldsymbol{R} \tilde{\boldsymbol{\beta}}-\boldsymbol{r})^{T}\{\operatorname{Var}(\boldsymbol{R} \hat{\boldsymbol{\beta}})\}^{-1}(\boldsymbol{R} \tilde{\boldsymbol{\beta}}-\boldsymbol{r}) \\
& =(\boldsymbol{R} \tilde{\boldsymbol{\beta}}-\boldsymbol{r})^{T}\left\{\boldsymbol{R}\left(\boldsymbol{X}^{T} \boldsymbol{\Sigma}^{-1} \boldsymbol{X}\right)^{-1} \boldsymbol{R}^{T}\right\}^{-1}(\boldsymbol{R} \tilde{\boldsymbol{\beta}}-\boldsymbol{r}) .
\end{aligned}
$$

Mas, $\boldsymbol{R} \overline{\boldsymbol{\beta}}=\boldsymbol{r}$ e usando (2.3.5) temos que

$$
\begin{aligned}
(\boldsymbol{R} \tilde{\boldsymbol{\beta}}-r) & =\boldsymbol{R} \tilde{\boldsymbol{\beta}}-\boldsymbol{R} \overline{\boldsymbol{\beta}} \\
& =\boldsymbol{R}(\tilde{\boldsymbol{\beta}}-\overline{\boldsymbol{\beta}}) \\
& =\frac{1}{2} \boldsymbol{R}\left(\boldsymbol{X}^{T} \boldsymbol{\Sigma}^{-1} \boldsymbol{X}\right)^{-1} \boldsymbol{R}^{T}(\tilde{\boldsymbol{\lambda}}-\bar{\lambda})
\end{aligned}
$$

e portanto segue que

$$
\xi_{w}=\frac{1}{4}(\tilde{\boldsymbol{\lambda}}-\bar{\lambda})^{T} \boldsymbol{R}\left(\boldsymbol{X} \boldsymbol{\Sigma}^{-1} \boldsymbol{X}\right)^{-1} \boldsymbol{R}^{T}(\tilde{\boldsymbol{\lambda}}-\bar{\lambda})=\xi_{k t}
$$

Com isso, verificamos que, para o cașo em que a variância do vetor de erros do modelo de regressão normal linear, $\Sigma$, é conhecida e queremos testar as hipóteses (2.4.1), nós definimos três estatísticas do teste, as quais satisfazem a seguinte relação:

$$
\xi_{r v}=\xi_{k t}=\xi_{w}
$$




\section{Caso II}

Por outro lado, a estatística da razão de verossimilhança para testar as hipóteses (2.4.2) é dada por

$$
\xi_{r v}=(\boldsymbol{y}-\boldsymbol{X} \tilde{\boldsymbol{\beta}})^{T} \boldsymbol{\Sigma}^{-1}(\boldsymbol{y}-\boldsymbol{X} \tilde{\boldsymbol{\beta}})-(\boldsymbol{y}-\boldsymbol{X} \hat{\boldsymbol{\beta}})^{T} \boldsymbol{\Sigma}^{-1}(\boldsymbol{y}-\boldsymbol{X} \hat{\boldsymbol{\beta}}),
$$

ou seja, $\xi_{r v}$ é o valor ótimo do seguinte PPQ:

$$
\left\{\begin{array}{l}
\min _{\boldsymbol{\beta}}\left\{(\boldsymbol{y}-\boldsymbol{X} \boldsymbol{\beta})^{T} \boldsymbol{\Sigma}^{-1}(\boldsymbol{y}-\boldsymbol{X} \boldsymbol{\beta})-(\boldsymbol{y}-\boldsymbol{X} \hat{\boldsymbol{\beta}})^{T} \Sigma^{-1}(\boldsymbol{y}-\boldsymbol{X} \hat{\boldsymbol{\beta}})\right\} \\
\text { sujeito a } \boldsymbol{R} \boldsymbol{\beta} \geq \boldsymbol{r} .
\end{array}\right.
$$

Usando o fato de que $\hat{\boldsymbol{\beta}}=\left(\boldsymbol{X} \boldsymbol{\Sigma}^{-1} \boldsymbol{X}\right)^{-1} \boldsymbol{X}^{T} \boldsymbol{\Sigma}^{-1} \boldsymbol{y}$ o PPQ anterior pode ser escrito como

$$
\left\{\begin{array}{l}
\min _{\boldsymbol{\beta}}\left\{\boldsymbol{y}^{T} \Sigma^{-1} \boldsymbol{X}\left(\boldsymbol{X}^{T} \Sigma^{-1} \boldsymbol{X}\right)^{-1} \boldsymbol{X}^{T} \Sigma^{-1} \boldsymbol{y}-2 \boldsymbol{y}^{T} \Sigma^{-1} \boldsymbol{X} \boldsymbol{\beta}+\right. \\
\left.+\boldsymbol{\beta}^{T} \boldsymbol{X}^{T} \Sigma^{-1} \boldsymbol{X} \boldsymbol{\beta}\right\} \text { sujeito a } \boldsymbol{R} \boldsymbol{\beta} \geq \boldsymbol{r}
\end{array}\right.
$$

e seguindo (2.4.6) e (2.4.7) o dual do PPQ (2.4.15) é dado por

$$
\left\{\begin{array}{l}
\max _{\lambda}\left\{\lambda^{T}(r-\boldsymbol{R} \hat{\boldsymbol{\beta}})-\frac{1}{4} \lambda^{T} \boldsymbol{R}\left(\boldsymbol{X}^{T} \boldsymbol{\Sigma}^{-1} \boldsymbol{X}\right)^{-1} \boldsymbol{R}^{T} \lambda\right\} \\
\text { sujeito a } \lambda \geq 0 .
\end{array}\right.
$$

Como foi definida anteriormente, a estatística de Kuhn-Tucker é o valor ótimo da função objetiva em (2.4.16), o qual é atingido em $\tilde{\lambda}$. Da relação (2.3.4) e do fato de que o ponto ótimo $(\tilde{\boldsymbol{\beta}}, \tilde{\boldsymbol{\lambda}})$ satisfaz $\tilde{\boldsymbol{\lambda}}^{T}(\boldsymbol{r}-\boldsymbol{R} \tilde{\boldsymbol{\beta}})=0$, temos que

$$
\begin{gathered}
\tilde{\lambda}^{T}\{\boldsymbol{r}-\boldsymbol{R} \hat{\boldsymbol{\beta}}-(\boldsymbol{R} \tilde{\boldsymbol{\beta}}-\boldsymbol{R} \hat{\boldsymbol{\beta}})\}=0, \\
\tilde{\lambda}^{T}(\boldsymbol{r}-\boldsymbol{R} \hat{\boldsymbol{\beta}})=\tilde{\lambda}^{T}(\boldsymbol{R} \tilde{\boldsymbol{\beta}}-\boldsymbol{R} \hat{\boldsymbol{\beta}}), \text { ou seja } \\
\tilde{\boldsymbol{\lambda}}^{T}(\boldsymbol{r}-\boldsymbol{R} \hat{\boldsymbol{\beta}})=\frac{1}{2} \tilde{\lambda} \boldsymbol{R}\left(\boldsymbol{X}^{T} \boldsymbol{\Sigma}^{-1} \boldsymbol{X}\right)^{-1} \boldsymbol{R}^{T} \tilde{\lambda}
\end{gathered}
$$

e portanto a estatística de Kuhn-Tucker fica dada por

$$
\xi_{k t}=\frac{1}{4} \tilde{\lambda}^{T} R\left(X^{T} \Sigma^{-1} \boldsymbol{X}\right)^{-1} \boldsymbol{R}^{T} \tilde{\lambda}
$$


Como estão satisfeitas as condições para que o primal e o dual tenham o mesmo valor ótimo, então $\xi_{r v}=\xi_{k t}$.

A estatística de Wald mede a distância entre o estimador restrito e o estimador não restrito, ou seja

$$
\begin{aligned}
\xi_{w} & =(\boldsymbol{R} \tilde{\boldsymbol{\beta}}-\boldsymbol{R} \hat{\boldsymbol{\beta}})^{T}\{\operatorname{Var}(\boldsymbol{R} \hat{\boldsymbol{\beta}})\}^{-1}(\boldsymbol{R} \tilde{\boldsymbol{\beta}}-\boldsymbol{R} \hat{\boldsymbol{\beta}}) \\
& =(\boldsymbol{R} \tilde{\boldsymbol{\beta}}-\boldsymbol{R} \hat{\boldsymbol{\beta}})^{T}\left\{\boldsymbol{R}\left(\boldsymbol{X}^{T} \boldsymbol{\Sigma}^{-1} \boldsymbol{X}\right)^{-1} \boldsymbol{R}^{T}\right\}^{-1}(\boldsymbol{R} \tilde{\boldsymbol{\beta}}-\boldsymbol{R} \hat{\boldsymbol{\beta}}) .
\end{aligned}
$$

E novamente da relação (2.3.4) temos que

$$
\boldsymbol{R} \tilde{\boldsymbol{\beta}}-\boldsymbol{R} \hat{\boldsymbol{\beta}}=\boldsymbol{R}(\tilde{\boldsymbol{\beta}}-\hat{\boldsymbol{\beta}})=\frac{1}{2} \boldsymbol{R}\left(\boldsymbol{X}^{T} \Sigma^{-1} \boldsymbol{X}\right)^{-1} \boldsymbol{R}^{T} \tilde{\boldsymbol{\lambda}}
$$

e portanto, substituindo a igualdade acima na estatística de Wald temos que

$$
\xi_{w}=\frac{1}{4} \tilde{\lambda}^{T} \boldsymbol{R}\left(\boldsymbol{X}^{T} \boldsymbol{\Sigma}^{-1} \boldsymbol{X}\right)^{-1} \boldsymbol{R}^{T} \tilde{\lambda}=\xi_{k t} .
$$

Portanto, no caso de testar as hipóteses (2.4.2), com $\boldsymbol{\Sigma}$ conhecida, as três estatísticas do teste também satifazem a relação

$$
\xi_{r v}=\xi_{k t}=\xi_{w}
$$

Observação: Wolak (1989) apresenta uma quarta estatística para o caso de testar as hipóteses (2.4.2) com $\Sigma$ conhecida. Aqui esta estatística será denotada por $\xi_{d}$ e vem do fato de que, sendo $\hat{\boldsymbol{\beta}}=\left(\boldsymbol{X} \boldsymbol{\Sigma}^{-1} \boldsymbol{X}\right)^{-1} \boldsymbol{X}^{T} \boldsymbol{\Sigma}^{-1} \boldsymbol{y}$, podemos escrever o PPQ (2.4.15) na seguinte forma:

$$
\min _{\boldsymbol{\beta}}\left\{(\boldsymbol{\beta}-\hat{\boldsymbol{\beta}})^{T}\left(\boldsymbol{X}^{T} \boldsymbol{\Sigma}^{-1} \boldsymbol{X}\right)^{-1}(\boldsymbol{\beta}-\hat{\boldsymbol{\beta}})\right\} \text { sujeito a } \boldsymbol{R} \boldsymbol{\beta} \geq \boldsymbol{r} .
$$

Esse PPQ é um caso especial da estatística da distância generalizada, derivada por Kodde e Palm (1986), a qual mede a distância entre o estimador não restrito e o estimador definido pela hipótese nula. Essa estatística será tratada com detalhes no Capítulo 3 . 
Por enquanto, definindo a estatística da distância $\xi_{d}$, como sendo o valor ótimo do PPQ (2.4.19) temos que

$$
\xi_{d}=(\tilde{\boldsymbol{\beta}}-\hat{\boldsymbol{\beta}})^{T}\left(\boldsymbol{X}^{T} \boldsymbol{\Sigma}^{-1} \boldsymbol{X}\right)^{-1}(\tilde{\boldsymbol{\beta}}-\hat{\boldsymbol{\beta}}) .
$$

Como o PPQ (2.4.19) é equivalente ao PPQ (2.4.15), eles têm o mesmo valor ótimo e portanto $\xi_{d}=\xi_{r v}$. Assim, verificamos que quando $\Sigma$ é conhecida, para testar as hipóteses (2.4.2) a seguinte relação fica satisfeita:

$$
\xi_{r v}=\xi_{k t}=\xi_{w}=\xi_{d}
$$

\subsection{Distribuição nula da estatística $\xi_{r v}$ quando $\Sigma$ é conhecida}

Na seção anterior foi mostrado que as três estatísticas $\xi_{r v}, \xi_{k t}$, e $\xi_{w}$, para testar as hipóteses (2.4.1) e (2.4.2) são iguais sob a suposição de que $\Sigma$ é conhecida e portanto têm a mesma distribuição sob a hipótese nula. Vamos então determinar a distribuição nula da estatística da razão de verossimilhança $\xi_{r v}$.

\section{Caso I}

Inicialmente, devemos observar que o problema de testar as hipóteses (2.4.1) está relacionado com o problema de testar

$$
H_{0}: \boldsymbol{\mu}=\mathbf{0} \text { versus } H_{1}: \boldsymbol{\mu} \geq \mathbf{0}
$$

com pelo menos uma desigualdade estrita em $H_{1}$, para uma amostra de uma variável aleatória $z \sim \mathrm{N}_{k}(\boldsymbol{\mu}, \mathrm{V})$, com $\mathrm{V}$ conhecida e positiva definida. Para ver isso, vamos 
mostrar a seguir que a estatística da razão de verossimilhança para testar as hipóteses (2.4.1) para o modelo

$$
\boldsymbol{y}=\boldsymbol{X} \boldsymbol{\beta}+\varepsilon, \quad \boldsymbol{R} \boldsymbol{\beta} \geq \boldsymbol{r}, \quad \boldsymbol{\varepsilon} \sim \mathrm{N}_{n}(\mathbf{0}, \boldsymbol{\Sigma}),
$$

é a mesma para modelo

$$
\hat{\boldsymbol{\mu}}=\boldsymbol{\mu}+\boldsymbol{v}, \quad \boldsymbol{\mu} \geq 0, \quad \boldsymbol{v} \sim \mathrm{N}_{k}(0, \mathrm{~V}),
$$

onde $\boldsymbol{\mu}=\boldsymbol{R} \boldsymbol{\beta}-\boldsymbol{r}, \mathrm{V}=\boldsymbol{R}\left(\boldsymbol{Y}^{\top} \mathrm{E} \ldots \ldots\right.$ stimador não restrito de $\boldsymbol{\mu}$.

Observe que para o modelo (2.5.2), a estatístıca ... «ào de verossimilhança $\xi_{r v}$, é o valor ótimo do seguinte $\mathrm{PPQ}$ :

$$
\left\{\begin{array}{l}
\min _{\boldsymbol{\beta}}\left\{(\boldsymbol{y}-\boldsymbol{X} \boldsymbol{\beta})^{T} \boldsymbol{\Sigma}^{-1}(\boldsymbol{y}-\boldsymbol{X} \boldsymbol{\beta})-(\boldsymbol{y}-\boldsymbol{X} \overline{\boldsymbol{\beta}})^{T} \Sigma^{-1}(\boldsymbol{y}-\boldsymbol{X} \overline{\boldsymbol{\beta}})\right\} \\
\text { sujeito a } \boldsymbol{R} \boldsymbol{\beta} \geq \boldsymbol{r} .
\end{array}\right.
$$

Agora

$$
(\boldsymbol{y}-\boldsymbol{X} \boldsymbol{\beta})=\boldsymbol{y}-\boldsymbol{X} \hat{\boldsymbol{\beta}}+\boldsymbol{X} \hat{\boldsymbol{\beta}}-\boldsymbol{X} \boldsymbol{\beta}=(\boldsymbol{y}-\boldsymbol{X} \hat{\boldsymbol{\beta}})+\boldsymbol{X}(\hat{\boldsymbol{\beta}}-\boldsymbol{\beta}),
$$

e como

$$
\begin{aligned}
(\boldsymbol{y}-\boldsymbol{X} \hat{\boldsymbol{\beta}})^{T} & \boldsymbol{\Sigma}^{-1} \boldsymbol{X}(\hat{\boldsymbol{\beta}}-\boldsymbol{\beta})= \\
& =\boldsymbol{y}^{T} \boldsymbol{\Sigma}^{-1} \boldsymbol{X} \hat{\boldsymbol{\beta}}-y^{T} \Sigma^{-1} \boldsymbol{X} \boldsymbol{\beta}-\hat{\boldsymbol{\beta}}^{T} \boldsymbol{X}^{T} \Sigma^{-1} \boldsymbol{X} \hat{\boldsymbol{\beta}}+\hat{\boldsymbol{\beta}}^{T} \boldsymbol{X}^{T} \boldsymbol{\Sigma}^{-1} \boldsymbol{X} \boldsymbol{\beta} \\
& =\boldsymbol{y}^{T} \boldsymbol{\Sigma}^{-1} \boldsymbol{X} \hat{\boldsymbol{\beta}}-\boldsymbol{y}^{T} \boldsymbol{\Sigma}^{-1} \boldsymbol{X} \boldsymbol{\beta}-\boldsymbol{y}^{T} \boldsymbol{\Sigma}^{-1} \boldsymbol{X} \hat{\boldsymbol{\beta}}+\boldsymbol{y}^{T} \boldsymbol{\Sigma}^{-1} \boldsymbol{X} \boldsymbol{\beta}=\mathbf{0},
\end{aligned}
$$

então

$$
\begin{aligned}
(\boldsymbol{y}-\boldsymbol{X} \boldsymbol{\beta})^{T} & \boldsymbol{\Sigma}^{-1}(\boldsymbol{y}-\boldsymbol{X} \boldsymbol{\beta})= \\
& =\{(\boldsymbol{y}-\boldsymbol{X} \hat{\boldsymbol{\beta}})+\boldsymbol{X}(\hat{\boldsymbol{\beta}}-\boldsymbol{\beta})\}^{T} \boldsymbol{\Sigma}^{-1}\{(\boldsymbol{y}-\boldsymbol{X} \hat{\boldsymbol{\beta}})+\boldsymbol{X}(\hat{\boldsymbol{\beta}}-\boldsymbol{\beta})\} \\
& =(\boldsymbol{y}-\boldsymbol{X} \hat{\boldsymbol{\beta}})^{T} \boldsymbol{\Sigma}^{-1}(\boldsymbol{y}-\boldsymbol{X} \hat{\boldsymbol{\beta}})+(\hat{\boldsymbol{\beta}}-\boldsymbol{\beta})^{T} \boldsymbol{X}^{T} \boldsymbol{\Sigma}^{-1} \boldsymbol{X}(\hat{\boldsymbol{\beta}}-\boldsymbol{\beta}) .
\end{aligned}
$$

Assim o PPQ (2.5.4) torna-se

$$
\left\{\begin{array}{l}
\min _{\boldsymbol{\beta}}\left\{(\hat{\boldsymbol{\beta}}-\boldsymbol{\beta})^{T} \boldsymbol{X}^{T} \Sigma^{-1} \boldsymbol{X}(\hat{\boldsymbol{\beta}}-\boldsymbol{\beta})+(\boldsymbol{y}-\boldsymbol{X} \hat{\boldsymbol{\beta}})^{T} \Sigma^{-1}(\boldsymbol{y}-\boldsymbol{X} \hat{\boldsymbol{\beta}})-\right. \\
\left.-(\boldsymbol{y}-\boldsymbol{X} \overline{\boldsymbol{\beta}})^{T} \boldsymbol{\Sigma}^{-1}(\boldsymbol{y}-\boldsymbol{X} \overline{\boldsymbol{\beta}})\right\} \quad \text { sujeito a } \boldsymbol{R} \boldsymbol{\beta} \geq \boldsymbol{r}
\end{array}\right.
$$


Mas,

$$
\begin{aligned}
& \quad(\boldsymbol{y}-\boldsymbol{X} \hat{\boldsymbol{\beta}})^{T} \Sigma^{-1}(\boldsymbol{y}-\boldsymbol{X} \hat{\boldsymbol{\beta}})-(\boldsymbol{y}-\boldsymbol{X} \overline{\boldsymbol{\beta}})^{T} \Sigma^{-1}(\boldsymbol{y}-\boldsymbol{X} \overline{\boldsymbol{\beta}})= \\
& =+\hat{\boldsymbol{\beta}}^{T} \boldsymbol{X}^{T} \Sigma^{-1} \boldsymbol{X} \hat{\boldsymbol{\beta}}-2 \boldsymbol{y}^{T} \Sigma^{-1} \boldsymbol{X} \hat{\boldsymbol{\beta}}-\overline{\boldsymbol{\beta}}^{T} \boldsymbol{X}^{T} \Sigma^{-1} \boldsymbol{X} \overline{\boldsymbol{\beta}}+2 y^{T} \Sigma^{-1} \boldsymbol{X} \overline{\boldsymbol{\beta}} \\
& =-\hat{\boldsymbol{\beta}}^{T} \boldsymbol{X}^{T} \Sigma^{-1} \boldsymbol{X} \hat{\boldsymbol{\beta}}-\overline{\boldsymbol{\beta}}^{T} \boldsymbol{X}^{T} \Sigma^{-1} \boldsymbol{X} \overline{\boldsymbol{\beta}}+2 \hat{\boldsymbol{\beta}}^{T} \boldsymbol{X}^{T} \Sigma^{-1} \boldsymbol{X} \hat{\boldsymbol{\beta}}-2 \boldsymbol{y}^{T} \Sigma^{-1} \boldsymbol{X} \hat{\boldsymbol{\beta}}+2 \boldsymbol{y}^{T} \Sigma^{-1} \boldsymbol{X} \overline{\boldsymbol{\beta}} \\
& =-\hat{\boldsymbol{\beta}}^{T} \boldsymbol{X}^{T} \Sigma^{-1} \boldsymbol{X} \hat{\boldsymbol{\beta}}-\overline{\boldsymbol{\beta}}^{T} \boldsymbol{X}^{T} \Sigma^{-1} \boldsymbol{X} \overline{\boldsymbol{\beta}}+2 \boldsymbol{y}^{T} \boldsymbol{\Sigma}^{-1} \boldsymbol{X} \hat{\boldsymbol{\beta}}-2 \boldsymbol{y}^{T} \Sigma^{-1} \boldsymbol{X} \hat{\boldsymbol{\beta}}+2 \hat{\boldsymbol{\beta}}^{T} \boldsymbol{X}^{T} \boldsymbol{\Sigma}^{-1} \boldsymbol{X} \overline{\boldsymbol{\beta}} \\
& =-(\hat{\boldsymbol{\beta}}-\overline{\boldsymbol{\beta}})^{T} \boldsymbol{X}^{T} \boldsymbol{\Sigma}^{-1} \boldsymbol{X}(\hat{\boldsymbol{\beta}}-\overline{\boldsymbol{\beta}}) .
\end{aligned}
$$

Com isso, podemos ver que o PPQ (2.5.5) é equivalente a

$$
\left\{\begin{array}{l}
\min _{\boldsymbol{\beta}}\left\{(\hat{\boldsymbol{\beta}}-\boldsymbol{\beta})^{T} \boldsymbol{X}^{T} \Sigma^{-1} \boldsymbol{X}(\hat{\boldsymbol{\beta}}-\boldsymbol{\beta})-(\hat{\boldsymbol{\beta}}-\overline{\boldsymbol{\beta}})^{T} \boldsymbol{X}^{T} \Sigma^{-1} \boldsymbol{X}(\hat{\boldsymbol{\beta}}-\overline{\boldsymbol{\beta}})\right\} \\
\text { sujeito a } \boldsymbol{R} \boldsymbol{\beta} \geq r
\end{array}\right.
$$

Note que o valor ótimo do PPQ (2.5.6) é a estatística da razão de verossimilhança para o modelo $\hat{\boldsymbol{\beta}}=\boldsymbol{\beta}+\mathrm{u}$ sujeito à restrição $\boldsymbol{R} \boldsymbol{\beta} \geq \boldsymbol{r}$, com $\mathrm{u} \sim \mathrm{N}_{p}\left\{\mathbf{0},\left(\boldsymbol{X}^{T} \boldsymbol{\Sigma}^{-1} \boldsymbol{X}\right)^{-1}\right\}$. Pré-multiplicando esse modelo por $\boldsymbol{R}$ e subtraindo $r$ em cada membro da igualdade obtém-se o modelo $\boldsymbol{R} \hat{\boldsymbol{\beta}}-\boldsymbol{r}=\boldsymbol{R} \boldsymbol{\beta}-\boldsymbol{r}+\boldsymbol{R u}$ sujeito à restrição $\boldsymbol{R} \boldsymbol{\beta}-\boldsymbol{r} \geq \mathbf{0}$, com $\boldsymbol{R u} \sim \mathrm{N}_{k}(\mathbf{0}, \mathrm{V})$, o qual torna-se o modelo (2.5.3) fazendo-se $\boldsymbol{\mu}=\boldsymbol{R} \boldsymbol{\beta}-\boldsymbol{r}$ e $\boldsymbol{R u}=\boldsymbol{v}$. O PPQ desse último modelo é dado por

$$
\min _{\mu}\left\{(\hat{\mu}-\mu)^{T} \mathrm{~V}^{-1}(\hat{\mu}-\mu) \text { sujeito a } \boldsymbol{\mu} \geq 0\right\}
$$

Como $\boldsymbol{R}$ é de posto completo e pela invariância da estimativa de máxima verossimilhança sob esse tipo de transformação o PPQ acima levará à mesma solução do PPQ (2.5.6) (veja, por exemplo, Wolak, 1988). Logo, a estatística da razão de verossimilhança será a mesma para testar as hipóteses (2.4.1) sob os modelos (2.5.2) e (2.5.3).

Dessa forma, para o nosso propósito, basta determinar a distribuição nula da estatística da razão de verossimilhança para testar as hipóteses $H_{0}: \boldsymbol{\mu}=\mathbf{0}$ versus $H_{1}: \boldsymbol{\mu} \geq \mathbf{0}$ 
com base em uma amostra de uma variável aleatória $z \sim N_{k}(\boldsymbol{\mu}, \mathrm{V})$, com $\mathrm{V}$ conhecida e positiva definida.

Para isso, seja V uma matriz $(k \times k)$ positiva definida e $\mathbf{u}$ um vetor $(k \times 1)$ de constantes. A função

$$
f(\boldsymbol{\mu})=2 \mathbf{u}^{T} \boldsymbol{\mu}+\boldsymbol{\mu}^{T} \mathbf{V} \boldsymbol{\mu}
$$

é uma forma quadrática convexa em $\boldsymbol{\mu}$ e o problema é minimizar $f(\boldsymbol{\mu})$ sujeito a $\boldsymbol{\mu} \geq 0$. Uma condição necessária e suficiente para que um vetor $\tilde{\mu}$ seja ótimo é (veja, por exemplo. Nüesch, 1966)

$$
\left(\mathbf{u}^{T}+\tilde{\mu}^{T} \mathrm{~V}\right) \tilde{\mu} \leq\left(\mathbf{u}^{T}+\tilde{\mu}^{T} \mathrm{~V}\right) \boldsymbol{\mu}, \quad \forall \boldsymbol{\mu} \geq 0
$$

Essa condição será usada para provar o seguinte lema:

Lema 2.1. O vetor $\tilde{\boldsymbol{\mu}}$ é ótimo se, e somente se,

(a) $u_{j}+\tilde{\mu}^{T} \mathrm{~V}_{j} \geq 0$;

(b) $u_{j}+\tilde{\mu}^{T} \mathbf{V}_{j}>0 \Longrightarrow \tilde{\mu}_{j}=0, \forall j$, onde $u_{j}$ é o j-ésimo componente de $\mathbf{u} e \mathbf{V}_{j}$ é a j-ésima coluna de $\mathbf{V}$.

Prova. Suponha que para algum $j,\left(u_{j}+\tilde{\mu}^{T} \mathbf{V}_{j}\right)<0$. Seja $\mu_{j}=a$ e $\mu_{i}=0, \forall i \neq j$. Então $\left(u_{j}+\tilde{\boldsymbol{\mu}}^{T} \mathbf{V}\right) \boldsymbol{\mu}=\left(u_{j}+\tilde{\boldsymbol{\mu}}^{T} \mathbf{V}_{j}\right) a$. Fazendo $a \rightarrow+\infty$, isto implica em $\left(u_{j}+\tilde{\boldsymbol{\mu}}^{T} \mathbf{V}_{j}\right) \rightarrow$ $-\infty$, o que torna impossível a desigualdade (2.5.7), isso prova o item (a) do Lema 2.1.

Suponha agora que $\left(u_{j}+\tilde{\mu}^{T} \mathbf{V}_{j}\right)>0$ e assuma que $\tilde{\mu}_{j}>0$. Seja $\mu_{i}=0, \forall i \neq j$ e $\mu_{j}=\tilde{\mu}_{j} / 2$. Portanto,

$$
\left(\mathbf{u}^{T}+\tilde{\boldsymbol{\mu}}^{T} \mathbf{V}\right) \tilde{\boldsymbol{\mu}} \geq\left(u_{j}+\tilde{\boldsymbol{\mu}}^{T} \mathbf{V}_{j}\right) \tilde{\mu}_{j}>\left(u_{j}+\tilde{\mu}^{T} \mathbf{V}_{j}\right) \mu_{j}=\left(\mathbf{u}^{T}+\tilde{\mu}^{T} \mathbf{V}\right) \boldsymbol{\mu}
$$

o que contradiz a desigualdade (2.5.7). Assim o item (b) do lema fica provado. 
Para provar a volta, note que os itens (a) e (b) do Lema 2.1 implicam em

$$
\left(\mathbf{u}^{T}+\tilde{\mu}^{T} \mathrm{~V}\right) \tilde{\mu}=0
$$

Como

$$
\left(\mathbf{u}^{T}+\tilde{\mu}^{T} \mathbf{V}\right) \tilde{\boldsymbol{\mu}}=\sum_{j=1}^{k}\left(u_{j}+\tilde{\mu}^{T} \mathrm{~V}_{j}\right) \tilde{\mu}_{j}
$$

e para cada $j$ um dos fatores é zero, isso implica em

$$
0 \leq\left(\mathbf{u}^{T}+\tilde{\mu}^{T} \mathbf{V}\right) \mu, \quad \forall \mu \geq 0
$$

Da igualdade (2.5.8) temos que

$$
\left(\mathbf{u}^{T}+\tilde{\mu}^{T} \mathrm{~V}\right) \tilde{\mu} \leq\left(\mathbf{u}^{T}+\tilde{\mu}^{T} \mathrm{~V}\right) \boldsymbol{\mu}, \quad \forall \boldsymbol{\mu} \geq 0,
$$

logo $\tilde{\boldsymbol{\mu}}$ é ótimo pela desigualdade (2.5.7).

Usando a igualdade (2.5.8), o valor mínimo de $f$ é dado por

$$
\begin{aligned}
f(\tilde{\boldsymbol{\mu}}) & =2 \mathbf{u}^{T} \tilde{\boldsymbol{\mu}}+\tilde{\boldsymbol{\mu}}^{T} \mathrm{~V} \tilde{\boldsymbol{\mu}} \\
& =2 \mathbf{u}^{T} \tilde{\boldsymbol{\mu}}+2 \tilde{\boldsymbol{\mu}}^{T} \mathrm{~V} \tilde{\boldsymbol{\mu}}-\tilde{\boldsymbol{\mu}}^{T} \mathrm{~V} \tilde{\boldsymbol{\mu}} \\
& =2\left(\mathbf{u}^{T}+\tilde{\boldsymbol{\mu}}^{T} \mathrm{~V}\right) \tilde{\boldsymbol{\mu}}-\tilde{\boldsymbol{\mu}}^{T} \mathrm{~V} \tilde{\boldsymbol{\mu}} \\
& =-\tilde{\boldsymbol{\mu}}^{T} \mathrm{~V} \tilde{\boldsymbol{\mu}} .
\end{aligned}
$$

Teorema 2.1. O teste da razão de verossimilhança para testar $H_{0}: \boldsymbol{\mu}=0$ contra $H_{1}: \boldsymbol{\mu} \geq \mathbf{0}$, rejeita $H_{0}$ quando $\tilde{\boldsymbol{\mu}}^{T} \mathrm{~V}^{-1} \tilde{\boldsymbol{\mu}} \geq c$, onde $c>0$ e $\tilde{\boldsymbol{\mu}}$ maximiza a função de verossimilhança sujeito à restrição $\mu \geq 0$.

Prova. Para uma amostra de uma variável aleatória $z \sim \mathrm{N}_{k}(\boldsymbol{\mu}, \mathrm{V}), \mathrm{V}$ conhecida e positiva definida, a função de verossimilhança é dada por

$$
L(\boldsymbol{\mu})=(2 \pi)^{-k / 2}|\mathrm{~V}|^{-1 / 2} \exp \left\{-\frac{1}{2}(z-\mu)^{T} \mathrm{~V}^{-1}(z-\mu)\right\} .
$$


Sob $H_{0}$,

$$
\max _{\boldsymbol{\mu}=\mathbf{0}} L(\boldsymbol{\mu})=L(\mathbf{0})=(2 \pi)^{-k / 2}|\mathbf{V}|^{-1 / 2} \exp \left\{-\frac{1}{2} \boldsymbol{z}^{T} \mathbf{V}^{-1} \boldsymbol{z}\right\}
$$

Sob $H_{1}$,

$$
\begin{gathered}
\max _{\boldsymbol{\mu} \geq 0} L(\boldsymbol{\mu})= \\
=\max _{\boldsymbol{\mu} \geq \mathbf{0}}\left\{(2 \pi)^{-k / 2}|\mathbf{V}|^{-1 / 2} \exp \left[-\frac{1}{2} \boldsymbol{z}^{T} \mathbf{V}^{-1} \boldsymbol{z}+\left(-2 \boldsymbol{z}^{T} \mathbf{V}^{-1} \boldsymbol{\mu}+\boldsymbol{\mu}^{T} \mathrm{~V}^{-1} \boldsymbol{\mu}\right)\right]\right\},
\end{gathered}
$$

que é equivalente a

$$
\min _{\boldsymbol{\mu} \geq 0}\left\{-2 \boldsymbol{z}^{T} \mathbf{V}^{-1} \boldsymbol{\mu}+\boldsymbol{\mu}^{T} \mathbf{V}^{-1} \boldsymbol{\mu}\right\}=-\tilde{\boldsymbol{\mu}}^{T} \mathbf{V}^{-1} \tilde{\boldsymbol{\mu}}
$$

por (2.5.9). Portanto,

$$
\max _{\boldsymbol{\mu} \geq 0} L(\boldsymbol{\mu})=L(\tilde{\boldsymbol{\mu}})=(2 \pi)^{-k / 2}|\mathbf{V}|^{-1 / 2} \exp \left\{-\frac{1}{2} \boldsymbol{z}^{T} \mathbf{V}^{-1} \boldsymbol{z}-\tilde{\boldsymbol{\mu}}^{T} \mathbf{V}^{-1} \tilde{\boldsymbol{\mu}}\right\}
$$

Assim, temos que

$$
-2 \ln \left\{\frac{L(\mathbf{0})}{L(\tilde{\boldsymbol{\mu}})}\right\}=\tilde{\boldsymbol{\mu}}^{T} \mathbf{V}^{-1} \tilde{\boldsymbol{\mu}}
$$

Cada componente do vetor $\tilde{\boldsymbol{\mu}}$ é positiva ou nula, isso leva à partição do espaço amostral em $2^{k}$ regiões distintas. Essas $\left(\begin{array}{l}k \\ \ell\end{array}\right)$ regiões serão denotadas por $\mathcal{X}_{\ell}$ e contêm os $\tilde{\boldsymbol{\mu}}$ 's com exatamente $\ell$ componentes positivas. Podemos rearranjar as variáveis de modo que as $\ell$ componentes positivas de $\tilde{\boldsymbol{\mu}}$ sejam sempre as $\ell$ últimas. Vamos assumir então que

$$
\mathcal{X}_{\ell}=\left\{z ; \tilde{\mu}_{1}=0 \text { e } \tilde{\mu}_{2}>0\right\}
$$

onde $\tilde{\mu}=\left(\tilde{\mu}_{1}^{T}, \tilde{\mu}_{2}^{T}\right)^{T}$.

Teorema 2.2. Sob $H_{0}: \boldsymbol{\mu}=0$, a estatística do teste, $\tilde{\boldsymbol{\mu}}^{T} \mathbf{V}^{-1} \tilde{\boldsymbol{\mu}}$, tem distribuição dada por

$$
\begin{aligned}
P\left(\tilde{\boldsymbol{\mu}}^{T} \mathrm{~V}^{-1} \tilde{\boldsymbol{\mu}} \geq c\right) & =\sum_{\ell=1}^{k} w(k, \ell ; \mathrm{V}) P\left(\chi_{\ell}^{2} \geq c\right), \forall c>0 e \\
P\left(\tilde{\boldsymbol{\mu}}^{T} \mathrm{~V}^{-1} \tilde{\boldsymbol{\mu}}=0\right) & =w(k, 0 ; \mathbf{V}),
\end{aligned}
$$

onde cada peso $w(k, \ell ; \mathrm{V})$ é a probabilidade de $\tilde{\boldsymbol{\mu}}$ conter exatamente $\ell$ componentes positivas. 
Prova. Sejam $R_{c}$ a região crítica do teste e $\cup \mathcal{X}_{\ell}$ a união de todos os $\tilde{\mu}$ 's os quais contêm exatamente $\ell$ componentes positivas. Como os $\mathcal{X}_{\ell}$ formam uma partição do espaço amostral, então

$$
R_{c}=\bigcup_{\ell=0}^{k}\left\{R_{c} \cap\left(\cup \mathcal{X}_{\ell}\right)\right\}
$$

e portanto temos que

$$
\begin{aligned}
P\left(R_{c}\right) & =\sum_{\ell=0}^{k} P\left\{\cup\left(R_{c} \cap \mathcal{X}_{\ell}\right)\right\} \\
& =\sum_{\ell=1}^{k} \sum_{\mathcal{X}} P\left(R_{c} \cap \mathcal{X}_{\ell}\right) \\
& =\sum_{\ell=1}^{k} \sum_{\mathcal{X}_{\ell}} P\left(\mathcal{X}_{\ell}\right) P\left(R_{c} \mid \mathcal{X}_{\ell}\right) .
\end{aligned}
$$

Note que $R_{c} \cap \mathcal{X}_{0}=\emptyset$. A soma $\sum_{\mathcal{X}} P\left(\mathcal{X}_{\ell}\right)$ é a probabilidade da união de todos os $\tilde{\mu}$ 's com exatamente $\ell$ componentes positivas, isto é, $\sum_{\mathcal{X}} P\left(\mathcal{X}_{\ell}\right)=w(k, \ell ; \mathrm{V})$.

Agora, sob $H_{0}, \tilde{\mu}_{2}$ tem distribuição normal multivariada com média zero e matriz de covariância $\mathrm{V}_{22.1}=\mathrm{V}_{22}-\mathrm{V}_{21} \mathrm{~V}_{11}^{-1} \mathrm{~V}_{12}$. Isso implica que $\tilde{\mu}_{2}^{T} \mathrm{~V}_{22.1}^{-1} \tilde{\mu}_{2}$ tem distribuição $\chi_{\ell}^{2}$. Além disso, os eventos $\left\{\tilde{\mu}_{2}>0\right\}$ e $\left\{\tilde{\mu}_{2}^{T} \mathbf{V}_{22.1}^{-1} \tilde{\mu}_{2} \geq c\right\}$ são independentes, portanto

$$
\begin{aligned}
P\left(R_{c} \mid \mathcal{X}_{\ell}\right) & =P\left(\tilde{\boldsymbol{\mu}}^{T} \mathbf{V}^{-1} \tilde{\boldsymbol{\mu}} \geq c \mid\left\{\tilde{\mu}_{1}=0\right\} \cap\left\{\tilde{\mu}_{2}>0\right\}\right) \\
& =P\left(\tilde{\boldsymbol{\mu}}_{2}^{T} \mathbf{V}_{22.1}^{-1} \tilde{\mu}_{2} \geq c\right) \\
& =P\left(\chi_{\ell}^{2} \geq c\right) .
\end{aligned}
$$

Assim,

$$
P\left(R_{c}\right)=P\left(\tilde{\boldsymbol{\mu}}^{T} \mathbf{V}^{-1} \tilde{\boldsymbol{\mu}} \geq c\right)=\sum_{\ell=1}^{k} w(k, \ell ; \mathbf{V}) P\left(\chi_{\ell}^{2} \geq c\right)
$$

Devemos notar que $\chi_{\ell}^{2}$ para $\ell=0$ é simplesmente massa total na origem, de modo que, $P\left(\chi_{0}^{2} \geq c\right)=0$ para todo $c>0$. Portanto, para a distribuição nula $\forall c \geq 0$, a soma deve iniciar em $\ell=0$. A soma dos pesos $w(k, \ell ; \mathrm{V})$ para $\ell$ variando de 0 até $k$ é igual a 1. Os detalhes sobre o cálculo dos pesos são dados no Apêndice A. 


\section{Caso II}

Para o caso de testar as hipóteses (2.4.2), a determinação da distribuição nula da estatística do teste é mais complicada devido ao fato da hipótese nula não especificar um único valor para $\boldsymbol{\beta}$, é necessário apenas que $\boldsymbol{\beta}$ satisfaça um sistema de equações lineares. Similarmente ao que foi feito para o caso I, deveremos considerar agora o seguinte problema de teste de hipóteses:

$$
H_{1}: \boldsymbol{\mu} \geq \mathbf{0} \text { versus } H_{2}-H_{1},
$$

onde $H_{2}: \boldsymbol{\mu} \in \mathbb{R}^{k}$ com $\hat{\boldsymbol{\mu}}=\boldsymbol{\mu}+\boldsymbol{v}, \boldsymbol{v} \sim \mathrm{N}_{k}(\mathbf{0}, \mathrm{V})$ e $\mathrm{V}$ conhecida e positiva definida.

Na estrutura de Neyman-Pearson para este caso, o espaço paramétrico é $\Theta=\mathbb{R}^{k}$ e sejam $\Theta_{1}$ e $\Theta-\Theta_{1}$ os espaços paramétricos sob $H_{1}$ e $H_{2}-H_{1}$, respectivamente, sendo $\Theta-\Theta_{1}$ o complemento de $\Theta_{1}$ em relação a $\Theta$. Se a estatísitica do teste $\xi$ e a região crítica $S$ satisfazem

$$
\sup _{\boldsymbol{\mu} \in \Theta_{1}} P(\xi \in S \mid \boldsymbol{\mu}, \mathbf{V})=\alpha,
$$

então temos que $S$ é a região crítica para um teste de tamanho $\alpha$ para a hipótese $H_{1}$ (veja Lehmann, 1986).

Seguindo essa estrutura, vamos determinar uma região crítica de tamanho $\alpha$ para testar $H_{1}$ contra $H_{2}-H_{1}$. Note que a estatística da razão de verossimilhança para este problema é o valor ótimo da função objetiva do seguinte PPQ:

$$
\min _{\boldsymbol{\mu}}\left\{(\hat{\boldsymbol{\mu}}-\boldsymbol{\mu})^{T} \mathbf{V}^{-1}(\hat{\boldsymbol{\mu}}-\boldsymbol{\mu})\right\} \text { sujeito a } \boldsymbol{\mu} \geq \mathbf{0} .
$$

Sendo $\tilde{\boldsymbol{\mu}}$ o valor ótimo de $\boldsymbol{\mu}$ satisfazendo o PPQ (2.5.12), a estatística da razão de verossimilhança fica dada por

$$
\xi_{r v}=(\hat{\mu}-\tilde{\mu})^{T} V^{-1}(\hat{\mu}-\tilde{\mu}) .
$$


Lema 2.2. Para todo $\mu \geq 0$ e $c>0$,

$$
P\left(\xi_{r v} \geq c \mid \boldsymbol{\mu}, \mathrm{V}\right) \leq P\left(\xi_{r v} \geq c \mid \boldsymbol{\mu}=0, \mathrm{~V}\right)
$$

Prova. Esse lema é um caso particular do Lema 8.2 de Perlman (1969) com $\mu_{1}=0$, $\mu_{2}=\mu$ e $\mathcal{C}=\{\mu: \mu \geq 0\}$.

Do Lema 2.2 é imediato que $\sup _{\boldsymbol{\mu} \in \Theta_{1}} P\left(\xi_{r v} \geq c \mid \boldsymbol{\mu}, \mathrm{V}\right)=P\left(\xi_{r v} \geq c \mid \boldsymbol{\mu}=0, \mathrm{~V}\right)$, e portanto fica determinado o único valor menos favorável para encontrar a distribuição nula para um teste de tamanho $\alpha$ da hipótese nula composta $H_{1}$.

Usando (2.5.9) temos que $(\hat{\boldsymbol{\mu}}-\tilde{\boldsymbol{\mu}})^{T} \mathrm{~V}^{-1}(\hat{\boldsymbol{\mu}}-\tilde{\boldsymbol{\mu}})=\hat{\boldsymbol{\mu}}^{T} \mathrm{~V}^{-1} \hat{\boldsymbol{\mu}}-\tilde{\boldsymbol{\mu}}^{T} \mathrm{~V}^{-1} \tilde{\boldsymbol{\mu}}$ e pela independência das duas últimas formas quadráticas (veja, por exemplo, Wolak, 1989) segue do Teorema 2.2 que

$$
P\left(\xi_{r v} \geq c \mid \boldsymbol{\mu}=0, \mathrm{~V}\right)=\sum_{\ell=1}^{k} w(k, k-\ell ; \mathrm{V}) P\left(\chi_{\ell}^{2} \geq c\right),
$$

e isso prova o seguinte:

Teorema 2.3. Sob a hipótese nula $H_{1}: \boldsymbol{\mu} \geq \mathbf{0}$, a distribuição da estatística da razão de verossimilhança satisfaz, para todo $c>0$,

$$
\begin{aligned}
\sup _{\boldsymbol{\mu} \in \Theta_{\mathbf{1}}} P\left(\xi_{r v} \geq c \mid \boldsymbol{\mu}, \mathrm{V}\right) & =P\left(\xi_{r v} \geq c \mid \boldsymbol{\mu}=\mathbf{0}, \mathrm{V}\right) \\
& =\sum_{\ell=1}^{k} w(k, k-\ell ; \mathrm{V}) P\left(\chi_{\ell}^{2} \geq c\right) \mathrm{e} \\
\sup _{\boldsymbol{\mu} \in \Theta_{1}} P\left(\xi_{r v}=0 \mid \boldsymbol{\mu}, \mathrm{V}\right) & =P\left(\xi_{r v}=0 \mid \boldsymbol{\mu}=0, \mathrm{~V}\right) \\
& =w(k, k ; \mathrm{V}) .
\end{aligned}
$$

Note que os pesos $w(k, \ell ; \mathrm{V})$ 's para os casos I e II, podem ser expressos na forma original substituindo-se a matriz $\mathrm{V}$ por $\boldsymbol{R}\left(\boldsymbol{X}^{T} \boldsymbol{\Sigma}^{-1} \boldsymbol{X}\right)^{-1} \boldsymbol{R}^{T}$. 


\section{$2.6 \quad \Sigma$ desconhecida}

Tipicamente, a matriz de covariância $\Sigma$ é desconhecida, sendo seus elementos funções contínuas de um número finito de parâmetros, os quais não estão relacionados com $\boldsymbol{\beta}$. Vamos assumir então que $\Sigma=\sigma^{2} \Omega$, com $\sigma^{2}$ e $\Omega$ desconhecidos e $\Omega$ positiva definida. Além disso, vamos supor a existência de estimadores de máxima verossimilhança para $\sigma^{2}$ e $\Omega$ sob as hipóteses $H_{0}, H_{1}$ e $H_{2}$ definidas em (2.4.1) e (2.4.2), os quais serão denotados, respectivamente, por $\left(\bar{\sigma}^{2}, \bar{\Omega}\right),\left(\tilde{\sigma}^{2}, \tilde{\Omega}\right)$ e $\left(\hat{\sigma}^{2}, \hat{\Omega}\right)$.

\subsubsection{Definição das estatísticas}

\section{Caso I}

Para testar as hipóteses

$$
H_{0}: \boldsymbol{R} \boldsymbol{\beta}=\boldsymbol{r} \text { versus } H_{1}: \boldsymbol{R} \boldsymbol{\beta} \geq \boldsymbol{r}
$$

com pelo menos uma desigualdade estrita em $H_{1}$, no modelo $\boldsymbol{y}=\boldsymbol{X} \boldsymbol{\beta}+\boldsymbol{\varepsilon}$, com $\boldsymbol{\varepsilon} \sim$ $\mathrm{N}_{n}\left(0, \sigma^{2} \Omega\right), \sigma^{2}$ e $\Omega$ desconhecidos com $\Omega$ positiva definida, a função de verossimilhança é dada por

$$
L\left(\boldsymbol{\beta}, \sigma^{2}, \boldsymbol{\Omega}\right)=\left(2 \pi \sigma^{2}\right)^{-n / 2}|\boldsymbol{\Omega}|^{-1 / 2} \exp \left\{\frac{-1}{2 \sigma^{2}}(\boldsymbol{y}-\boldsymbol{X} \boldsymbol{\beta})^{T} \boldsymbol{\Omega}^{-1}(\boldsymbol{y}-\boldsymbol{X} \boldsymbol{\beta})\right\} .
$$

Nesse caso os estimadores de máxima verossimilhança de $\sigma^{2}$ sob $H_{0}$ e $H_{1}$ são dados, respectivamente, por

$$
\begin{aligned}
\bar{\sigma}^{2} & =\frac{1}{n}(\boldsymbol{y}-\boldsymbol{X} \overline{\boldsymbol{\beta}})^{T} \overline{\boldsymbol{\Omega}}^{-1}(\boldsymbol{y}-\boldsymbol{X} \overline{\boldsymbol{\beta}}) \mathrm{e} \\
\tilde{\sigma}^{2} & =\frac{1}{n}(\boldsymbol{y}-\boldsymbol{X} \tilde{\boldsymbol{\beta}})^{T} \tilde{\boldsymbol{\Omega}}^{-1}(\boldsymbol{y}-\boldsymbol{X} \tilde{\boldsymbol{\beta}}) .
\end{aligned}
$$


Assim, o máximo de $L\left(\boldsymbol{\beta}, \sigma^{2}, \Omega\right)$ sob $H_{0}$ e $H_{1}$ ficam dados, respectivamente, por

$$
\begin{aligned}
& L\left(\overline{\boldsymbol{\beta}}, \bar{\sigma}^{2}, \overline{\boldsymbol{\Omega}}\right)=(2 \pi)^{-n / 2}\left(\bar{\sigma}^{2}\right)^{-n / 2}|\bar{\Omega}|^{-1 / 2} \exp \{-n / 2\} \mathrm{e} \\
& L\left(\tilde{\boldsymbol{\beta}}, \tilde{\sigma}^{2}, \tilde{\boldsymbol{\Omega}}\right)=(2 \pi)^{-n / 2}\left(\tilde{\sigma}^{2}\right)^{-n / 2}|\tilde{\boldsymbol{\Omega}}|^{-1 / 2} \exp \{-n / 2\} .
\end{aligned}
$$

Portanto, a estatística da razão de verossimilhança fica dada por

$$
\begin{aligned}
\xi_{r v} & =-2 \ln \left\{L\left(\overline{\boldsymbol{\beta}}, \bar{\sigma}^{2}, \bar{\Omega}\right) / L\left(\tilde{\boldsymbol{\beta}}, \tilde{\sigma}^{2}, \tilde{\Omega}\right)\right\} \\
& =-2 \ln \left\{\left(\bar{\sigma}^{2}\right)^{-n / 2}|\bar{\Omega}|^{-1 / 2} /\left(\tilde{\sigma}^{2}\right)^{-n / 2}|\tilde{\Omega}|^{-1 / 2}\right\} \\
& =-2 \ln \left\{\bar{\sigma}^{2}|\bar{\Omega}|^{1 / n} / \tilde{\sigma}^{2}|\tilde{\Omega}|^{1 / n}\right\}^{-n / 2} \\
& =n \ln \{V(0,0) / V(1,1)\},
\end{aligned}
$$

onde $V(0,0)=\bar{\sigma}^{2}|\bar{\Omega}|^{1 / n}$ e $V(1,1)=\tilde{\sigma}^{2}|\tilde{\Omega}|^{1 / n}$.

A estatística de Wald é baseada no estimador restrito por desigualdade e é dada por

$$
\xi_{w}=\frac{1}{\tilde{\sigma}^{2}}(\boldsymbol{R} \tilde{\boldsymbol{\beta}}-\boldsymbol{r})^{T}\left\{\boldsymbol{R}\left(\boldsymbol{X}^{T} \tilde{\boldsymbol{\Omega}}^{-1} \boldsymbol{X}\right) \boldsymbol{R}^{T}\right\}^{-1}(\boldsymbol{R} \tilde{\boldsymbol{\beta}}-\boldsymbol{r}) .
$$

Podemos ver que $\xi_{w}$ é o valor ótimo da função objetiva do PPQ (2.4.4) com $\Sigma$ trocado por $\tilde{\Sigma}=\tilde{\sigma}^{2} \tilde{\Omega}$, isto é

$$
\left\{\begin{array}{l}
\max _{\boldsymbol{\beta}}\left\{-\frac{1}{\bar{\sigma}^{2}}(\boldsymbol{y}-\boldsymbol{X} \boldsymbol{\beta})^{T} \tilde{\boldsymbol{\Omega}}^{-1}(\boldsymbol{y}-\boldsymbol{X} \boldsymbol{\beta})+\frac{1}{\tilde{\sigma}^{2}}\left(\boldsymbol{y}-\boldsymbol{X} \overline{\boldsymbol{\beta}}_{0}\right)^{T} \tilde{\boldsymbol{\Omega}}^{-1}\left(\boldsymbol{y}-\boldsymbol{X} \overline{\boldsymbol{\beta}}_{0}\right)\right\} \\
\text { sujeito a } \boldsymbol{R} \boldsymbol{\beta} \geq \boldsymbol{r},
\end{array}\right.
$$

e portanto, $\xi_{w}$ pode ser escrito como

$$
\begin{aligned}
\xi_{w} & =\frac{1}{\tilde{\sigma}^{2}}\left\{-(\boldsymbol{y}-\boldsymbol{X} \tilde{\boldsymbol{\beta}})^{T} \tilde{\boldsymbol{\Omega}}^{-1}(\boldsymbol{y}-\boldsymbol{X} \tilde{\boldsymbol{\beta}})+\left(\boldsymbol{y}-\boldsymbol{X} \overline{\boldsymbol{\beta}}_{0}\right)^{T} \tilde{\boldsymbol{\Omega}}^{-1}\left(\boldsymbol{y}-\boldsymbol{X} \overline{\boldsymbol{\beta}}_{0}\right)\right\} \\
& =-n+\frac{1}{\bar{\sigma}^{2}}\left(\boldsymbol{y}-\boldsymbol{X} \overline{\boldsymbol{\beta}}_{0}\right)^{T} \tilde{\boldsymbol{\Omega}}^{-1}\left(\boldsymbol{y}-\boldsymbol{X} \overline{\boldsymbol{\beta}}_{0}\right)
\end{aligned}
$$

onde $\overline{\boldsymbol{\beta}}_{0}$ é o estimador de máxima verossimilhança de $\boldsymbol{\beta}$ sujeito à restrição $\boldsymbol{R} \boldsymbol{\beta}=\boldsymbol{r}$ e usando a matriz de covariância $\tilde{\Sigma}=\tilde{\sigma}^{2} \tilde{\Omega}$.

Definindo

$$
V(0,1)=\frac{1}{n}\left(\boldsymbol{y}-\boldsymbol{X} \overline{\boldsymbol{\beta}}_{0}\right)^{T} \tilde{\Omega}^{-1}\left(\boldsymbol{y}-\boldsymbol{X} \overline{\boldsymbol{\beta}}_{0}\right)|\tilde{\boldsymbol{\Omega}}|^{1 / n}
$$


temos que

$$
\xi_{w}=-n+n \frac{V(0,1)}{V(1,1)}
$$

A estatística de Kuhn-Tucker definida em (2.4.11) é dada por

$$
\xi_{k t}=\frac{1}{4 \bar{\sigma}^{2}}(\tilde{\boldsymbol{\lambda}}-\bar{\lambda})^{T} \boldsymbol{R}\left(\boldsymbol{X}^{T} \bar{\Omega}^{-1} \boldsymbol{X}\right)^{-1} \boldsymbol{R}^{T}(\tilde{\boldsymbol{\lambda}}-\bar{\lambda}) .
$$

Devemos notar que $\xi_{k t}$ é também o valor ótimo do PPQ (2.4.4) com $\Sigma$ trocada por $\bar{\Sigma}=\bar{\sigma}^{2} \bar{\Omega}$, e portanto a estatística de Kuhn-Tucker pode ser escrita como

$$
\xi_{k t}=n-\frac{1}{\bar{\sigma}^{2}}\left(\boldsymbol{y}-\boldsymbol{X} \tilde{\boldsymbol{\beta}}_{1}\right)^{T} \overline{\boldsymbol{\Omega}}^{-1}\left(\boldsymbol{y}-\boldsymbol{X} \tilde{\boldsymbol{\beta}}_{1}\right),
$$

onde $\tilde{\boldsymbol{\beta}}_{1}$ é o estimador de máxima verossimilhança de $\boldsymbol{\beta}$ sujeito à restrição $\boldsymbol{R} \boldsymbol{\beta} \geq \boldsymbol{r}$ e usando a matriz de covariância $\bar{\Sigma}=\bar{\sigma}^{2} \bar{\Omega}$.

Definindo

$$
V(1,0)=\frac{1}{n}\left(\boldsymbol{y}-\boldsymbol{X} \tilde{\boldsymbol{\beta}}_{1}\right)^{T} \overline{\boldsymbol{\Omega}}^{-1}\left(\boldsymbol{y}-\boldsymbol{X} \tilde{\boldsymbol{\beta}}_{1}\right)|\overline{\boldsymbol{\Omega}}|^{-1 / n}
$$

temos que

$$
\xi_{k t}=n\left\{1-\frac{V(1,0)}{V(0,0)}\right\} .
$$

Para estabelecer a relação entre as estatísticas do teste das hipóteses (2.4.1) para o caso de $\sigma^{2}$ e $\Omega$ desconhecidos, que são similares às desigualdades obtidas por Berndt e Savin (1977), veja também Savin (1976) e Breusch (1979), vamos usar a seguinte desigualdade:

$$
\frac{x}{1+x} \leq \ln (1+x) \leq x \text { se } x \geq 0 .
$$

Além disso, $V(0,0)$ é obtido através da maximização sob $H_{0}$, do logaritmo da função de verossimilhança e $V(0,1)$ é obtido maximizando-se sob $H_{0}$, a mesma função com $\Omega$ trocado por $\tilde{\Omega}$ e como consequência temos que $V(0,0) \leq V(0,1)$ e $V(1,1) \leq V(1,0)$ (veja, por exemplo, Gouriéroux et al., 1982). 
Agora

$$
\begin{aligned}
\xi_{r v} & =n \ln \left\{\frac{V^{\cdot}(0.0)}{\Gamma^{\cdot}(1,1)}\right\}=n \ln \left\{1+\frac{I^{\cdot}(0,0)-I^{\cdot}(1,1)}{V^{\prime}(1,1)}\right\} \\
& \geq n\left\{1-\frac{V(1,1)}{V(0,0)}\right\} \geq n\left\{1-\frac{V(1,0)}{V(0,0)}\right\}=\xi_{k t} .
\end{aligned}
$$

Por outro lado,

$$
\begin{aligned}
& \xi_{r v}=n \ln \left\{\frac{V(0,0)}{V(1,1)}\right\}=n \ln \left\{1+\frac{V(0,0)-\Gamma(1,1)}{V(1,1)}\right\} \\
& \leq n\left\{\frac{V(0,0)-V(1,1)}{V(1,1)}\right\} \leq n\left\{\frac{V(0,1)}{V(1,1)}-1\right\} \\
& =-n+n \frac{V(0,1)}{V(1,1)}=\xi_{w} \text {. }
\end{aligned}
$$

Portanto, no caso em que $\sigma^{2}$ e $\Omega$ são desconhecidos e queremos testar as hipóteses (2.4.1), as três estatísticas do teste satisfazem a relação

$$
\xi_{k t} \leq \xi_{r v} \leq \xi_{w}
$$

\section{Caso II}

Para o caso de testar

$$
H_{1}: \boldsymbol{R} \boldsymbol{\beta} \geq \boldsymbol{r} \text { versus } H_{2}-H_{1}
$$

onde $H_{2}: \boldsymbol{\beta} \in \mathbb{R}^{k}$, a estatística da razão de verossimilhança é dada por

$$
\xi_{r v}=-2 \ln \left\{\frac{\max _{\mathbf{R} \boldsymbol{\beta} \geq \mathbf{r}, \sigma^{2}, \boldsymbol{\Omega}} L\left(\boldsymbol{\beta}, \sigma^{2}, \boldsymbol{\Omega}\right)}{\max _{\boldsymbol{\beta}, \sigma^{2}, \boldsymbol{\Omega}} L\left(\boldsymbol{\beta}, \sigma^{2}, \boldsymbol{\Omega}\right)}\right\} .
$$

A estatística de Wald definida em (2.4.18) com $\Sigma$ trocada por $\hat{\Sigma}=\hat{\sigma}^{2} \hat{\Omega}$ fica dada por

$$
\xi_{w}=\frac{1}{\hat{\sigma}^{2}}(\boldsymbol{R} \tilde{\boldsymbol{\beta}}-\boldsymbol{R} \hat{\boldsymbol{\beta}})^{T}\left\{\boldsymbol{R}\left(\boldsymbol{X}^{T} \hat{\Omega}^{-1} \boldsymbol{X}\right)^{-1} \boldsymbol{R}^{T}\right\}^{-1}(\boldsymbol{R} \tilde{\boldsymbol{\beta}}-\boldsymbol{R} \hat{\boldsymbol{\beta}}) .
$$

De (2.3.4) com $\Sigma$ trocada por $\hat{\Sigma}=\hat{\sigma}^{2} \hat{\Omega}$ temos que

$$
\tilde{\boldsymbol{\beta}}-\hat{\boldsymbol{\beta}}=\frac{\hat{\sigma}^{2}}{2}\left(\boldsymbol{X}^{T} \hat{\boldsymbol{\Omega}}^{-1} \boldsymbol{X}\right)^{-1} \boldsymbol{R}^{T} \tilde{\lambda}, \text { ou seja, }
$$




$$
\begin{gathered}
\boldsymbol{R} \tilde{\boldsymbol{\beta}}-\boldsymbol{R} \hat{\boldsymbol{\beta}}=\frac{\hat{\sigma}^{2}}{2} \boldsymbol{R}\left(\boldsymbol{X}^{T} \hat{\boldsymbol{\Omega}}^{-1} \boldsymbol{X}\right)^{-1} \boldsymbol{R}^{T} \tilde{\boldsymbol{\lambda}}, \text { de modo que, } \\
\tilde{\boldsymbol{\lambda}}=\frac{2}{\hat{\sigma}^{2}}\left\{\boldsymbol{R}\left(\boldsymbol{X}^{T} \hat{\boldsymbol{\Omega}}^{-1} \boldsymbol{X}\right)^{-1} \boldsymbol{R}^{T}\right\}^{-1}(\boldsymbol{R} \tilde{\boldsymbol{\beta}}-\boldsymbol{R} \hat{\boldsymbol{\beta}})
\end{gathered}
$$

o que implica em

$$
\begin{gathered}
\tilde{\boldsymbol{\beta}}=\hat{\boldsymbol{\beta}}+\left(\boldsymbol{X}^{T} \hat{\Omega}^{-1} \boldsymbol{X}\right)^{-1} \boldsymbol{R}\left\{\boldsymbol{R}\left(\boldsymbol{X}^{T} \hat{\Omega}^{-1} \boldsymbol{X}\right)^{-1} \boldsymbol{R}^{T}\right\}^{-1}(\boldsymbol{R} \tilde{\boldsymbol{\beta}}-\boldsymbol{R} \hat{\boldsymbol{\beta}}), \text { ou ainda } \\
(\boldsymbol{y}-\boldsymbol{X} \tilde{\boldsymbol{\beta}})=(\boldsymbol{y}-\boldsymbol{X} \hat{\boldsymbol{\beta}})-\boldsymbol{X}\left(\boldsymbol{X}^{T} \hat{\boldsymbol{\Omega}}^{-1} \boldsymbol{X}\right)^{-1} \boldsymbol{R}^{T}\left\{\boldsymbol{R}\left(\boldsymbol{X}^{T} \hat{\Omega}^{-1} \boldsymbol{X}\right)^{-1} \boldsymbol{R}^{T}\right\}^{-1}(\boldsymbol{R} \tilde{\boldsymbol{\beta}}-\boldsymbol{R} \hat{\boldsymbol{\beta}}) .
\end{gathered}
$$

Como

$$
\frac{1}{\hat{\sigma}^{2}} \boldsymbol{X}^{T} \hat{\Omega}^{-1}(\boldsymbol{y}-\boldsymbol{X} \hat{\boldsymbol{\beta}})=\frac{1}{\hat{\sigma}^{2}} \boldsymbol{X}^{T} \hat{\boldsymbol{\Omega}}^{-1} \boldsymbol{y}-\frac{1}{\hat{\sigma}^{2}} \boldsymbol{X}^{T} \hat{\Omega}^{-1} \boldsymbol{X} \hat{\boldsymbol{\beta}}=0
$$

então

$$
\begin{gathered}
\frac{1}{\hat{\sigma}^{2}}(\boldsymbol{y}-\boldsymbol{X} \tilde{\boldsymbol{\beta}})^{T} \hat{\boldsymbol{\Omega}}^{-1}(\boldsymbol{y}-\boldsymbol{X} \tilde{\boldsymbol{\beta}})= \\
=\frac{1}{\hat{\sigma}^{2}}(\boldsymbol{y}-\boldsymbol{X} \hat{\boldsymbol{\beta}})^{T} \hat{\boldsymbol{\Omega}}^{-1}(\boldsymbol{y}-\boldsymbol{X} \hat{\boldsymbol{\beta}})+\frac{1}{\hat{\sigma}^{2}}(\boldsymbol{R} \tilde{\boldsymbol{\beta}}-\boldsymbol{R} \hat{\boldsymbol{\beta}})^{T}\left\{\boldsymbol{R}\left(\boldsymbol{X}^{T} \hat{\boldsymbol{\Omega}}^{-1} \boldsymbol{X}\right)^{-1} \boldsymbol{R}^{T}\right\}^{-1}(\boldsymbol{R} \tilde{\boldsymbol{\beta}}-\boldsymbol{R} \hat{\boldsymbol{\beta}}),
\end{gathered}
$$

ou seja,

$$
\begin{aligned}
\xi_{w} & =\frac{1}{\hat{\sigma}^{2}}(\boldsymbol{y}-\boldsymbol{X} \tilde{\boldsymbol{\beta}})^{T} \hat{\boldsymbol{\Omega}}^{-1}(\boldsymbol{y}-\boldsymbol{X} \tilde{\boldsymbol{\beta}})-\frac{1}{\hat{\sigma}^{2}}(\boldsymbol{y}-\boldsymbol{X} \hat{\boldsymbol{\beta}})^{T} \hat{\boldsymbol{\Omega}}^{-1}(\boldsymbol{y}-\boldsymbol{X} \hat{\boldsymbol{\beta}}) \\
& =-2 \ln \left\{L\left(\tilde{\boldsymbol{\beta}}, \hat{\sigma}^{2}, \hat{\boldsymbol{\Omega}}\right) / L\left(\hat{\boldsymbol{\beta}}, \hat{\sigma}^{2}, \hat{\boldsymbol{\Omega}}\right)\right\} \\
& =-2 \ln \left\{\max _{\mathbf{R} \boldsymbol{\beta} \geq \mathbf{r}} L\left(\boldsymbol{\beta}, \hat{\sigma}^{2}, \hat{\boldsymbol{\Omega}}\right) / \max _{\boldsymbol{\beta}} L\left(\boldsymbol{\beta}, \hat{\sigma}^{2}, \hat{\boldsymbol{\Omega}}\right)\right\} .
\end{aligned}
$$

A estatística de Kuhn-Tuker definida em (2.4.17) com $\Sigma$ trocada por $\tilde{\Sigma}=\tilde{\sigma}^{2} \tilde{\Omega}$ fica dada por

$$
\xi_{k t}=\frac{\tilde{\sigma}^{2}}{4} \tilde{\lambda}^{T} \boldsymbol{R}\left(\boldsymbol{X}^{T} \tilde{\Omega}^{-1} \boldsymbol{X}\right)^{-1} \boldsymbol{R}^{T} \tilde{\lambda}
$$

De (2.3.4) com $\Sigma$ trocada por $\tilde{\Sigma}=\tilde{\sigma}^{2} \tilde{\Omega}$ temos que

$$
\tilde{\boldsymbol{\beta}}=\hat{\boldsymbol{\beta}}+\frac{\tilde{\sigma}^{2}}{2}\left(\boldsymbol{X}^{T} \tilde{\boldsymbol{\Omega}}^{-1} \boldsymbol{X}\right)^{-1} \boldsymbol{R}^{T} \tilde{\lambda}
$$

e portanto

$$
(\boldsymbol{y}-\boldsymbol{X} \tilde{\boldsymbol{\beta}})=(\boldsymbol{y}-\boldsymbol{X} \hat{\boldsymbol{\beta}})-\frac{\tilde{\sigma}^{2}}{2} \boldsymbol{X}\left(\boldsymbol{X}^{T} \tilde{\Omega}^{-1} \boldsymbol{X}\right)^{-1} \boldsymbol{R}^{T} \tilde{\lambda}
$$


Como

$$
\frac{1}{\tilde{\sigma}^{2}} \boldsymbol{X}^{T} \tilde{\Omega}^{-1}(\boldsymbol{y}-\boldsymbol{X} \hat{\boldsymbol{\beta}})=\frac{1}{\tilde{\sigma}^{2}} \boldsymbol{X}^{T} \tilde{\boldsymbol{\Omega}}^{-1} \boldsymbol{y}-\frac{1}{\tilde{\sigma}^{2}} \boldsymbol{X}^{T} \tilde{\Omega}^{-1} \boldsymbol{X} \hat{\boldsymbol{\beta}}=0
$$

temos que

$$
\begin{gathered}
\frac{1}{\tilde{\sigma}^{2}}(\boldsymbol{y}-\boldsymbol{X} \tilde{\boldsymbol{\beta}})^{T} \tilde{\boldsymbol{\Omega}}^{-1}(\boldsymbol{y}-\boldsymbol{X} \tilde{\boldsymbol{\beta}})= \\
=\frac{1}{\tilde{\sigma}^{2}}(\boldsymbol{y}-\boldsymbol{X} \hat{\boldsymbol{\beta}})^{T} \tilde{\boldsymbol{\Omega}}^{-1}(\boldsymbol{y}-\boldsymbol{X} \hat{\boldsymbol{\beta}})+\frac{\tilde{\sigma}^{2}}{4} \tilde{\boldsymbol{\lambda}}^{T} \boldsymbol{R}\left(\boldsymbol{X}^{T} \tilde{\boldsymbol{\Omega}}^{-1} \boldsymbol{X}\right)^{-1} \boldsymbol{R}^{T} \tilde{\boldsymbol{\lambda}}
\end{gathered}
$$

ou seja,

$$
\begin{aligned}
\xi_{k t} & =\frac{1}{\tilde{\sigma}^{2}}(\boldsymbol{y}-\boldsymbol{X} \tilde{\boldsymbol{\beta}})^{T} \tilde{\boldsymbol{\Omega}}^{-1}(\boldsymbol{y}-\boldsymbol{X} \tilde{\boldsymbol{\beta}})-\frac{1}{\tilde{\sigma}^{2}}(\boldsymbol{y}-\boldsymbol{X} \hat{\boldsymbol{\beta}})^{T} \tilde{\Omega}^{-1}(\boldsymbol{y}-\boldsymbol{X} \hat{\boldsymbol{\beta}}) \\
& =-2 \ln \left\{L\left(\tilde{\boldsymbol{\beta}}, \tilde{\sigma}^{2}, \tilde{\boldsymbol{\Omega}}\right) / L\left(\hat{\boldsymbol{\beta}}, \tilde{\sigma}^{2}, \tilde{\boldsymbol{\Omega}}\right)\right\} \\
& =-2 \ln \left\{\max _{\mathbf{R} \boldsymbol{\beta} \geq \mathbf{r}} L\left(\boldsymbol{\beta}, \tilde{\sigma}^{2}, \tilde{\boldsymbol{\Omega}}\right) / \max _{\boldsymbol{\beta}} L\left(\boldsymbol{\beta}, \tilde{\sigma}^{2}, \tilde{\boldsymbol{\Omega}}\right)\right\} .
\end{aligned}
$$

Das desigualdades de Breusch (1979), modificadas para este problema, temos que

$$
\begin{aligned}
\max _{\boldsymbol{\beta}} L\left(\boldsymbol{\beta} \mid \tilde{\sigma}^{2}, \tilde{\boldsymbol{\Omega}}\right) & \leq \max _{\boldsymbol{\beta}, \sigma^{2}, \boldsymbol{\Omega}} L\left(\boldsymbol{\beta}, \sigma^{2}, \boldsymbol{\Omega}\right)=\max _{\boldsymbol{\beta}} L\left(\boldsymbol{\beta} \mid \hat{\sigma}^{2}, \hat{\boldsymbol{\Omega}}\right) \mathrm{e} \\
\max _{\mathbf{R} \boldsymbol{\beta} \geq \mathbf{r}} L\left(\boldsymbol{\beta} \mid \hat{\sigma}^{2}, \hat{\boldsymbol{\Omega}}\right) & \leq \max _{\mathbf{R} \boldsymbol{\beta} \geq \mathbf{r}} L\left(\boldsymbol{\beta}, \sigma^{2}, \boldsymbol{\Omega}\right)=\max _{\mathbf{R} \boldsymbol{\beta} \geq \mathbf{r}} L\left(\boldsymbol{\beta} \mid \tilde{\sigma}^{2}, \tilde{\boldsymbol{\Omega}}\right) .
\end{aligned}
$$

Com isso, mostra-se que no caso de testar as hipóteses (2.4.2), com $\sigma^{2}$ e $\Omega$ desconhecidos, as três estatísticas do teste satisfazem a relação

$$
\xi_{k t} \leq \xi_{r v} \leq \xi_{w}
$$

Observação: A estatística da distância definida em (2.4.20), satisfaz

$$
\xi_{d}= \begin{cases}\xi_{w} & \text { se } \Sigma \text { for trocada por } \hat{\Sigma}=\hat{\sigma}^{2} \hat{\Omega} \\ \xi_{k t} & \text { se } \Sigma \text { for trocada por } \tilde{\Sigma}=\tilde{\sigma}^{2} \tilde{\Omega} .\end{cases}
$$

\subsubsection{Distribuição nula assintótica das estatísticas}

Vamos considerar agora a distribuição nula assintótica das estatísticas $\xi_{k t}, \xi_{r v}$ e $\xi_{w}$, assumindo que $\boldsymbol{\Sigma}=\sigma^{2} \Omega$, com $\sigma^{2}$ e $\Omega$ desconhecidas. 


\section{Caso I}

Para determinar a distribuição nula da estatística da razão de verossimilhança, vamos considerar inicialmente o caso de testar

$$
H_{0}: \boldsymbol{\mu}=\mathbf{0} \text { versus } H_{1}: \boldsymbol{\mu} \geq \mathbf{0}
$$

com pelo menos uma desigualdade estrita em $H_{1}$, para o modelo $\hat{\boldsymbol{\mu}}=\boldsymbol{\mu}+\boldsymbol{v}$, onde $\boldsymbol{\mu}=\boldsymbol{R} \boldsymbol{\beta}-\boldsymbol{r}, \operatorname{com} \boldsymbol{v} \sim \mathrm{N}_{k}\left(\mathbf{0}, \sigma^{2} \mathrm{~V}\right), \sigma^{2}$ desconhecido e $\mathrm{V}$ conhecida, positiva definida e dada por $\mathrm{V}=\boldsymbol{R}\left(\boldsymbol{X}^{T} \Omega^{-1} \boldsymbol{X}\right)^{-1} \boldsymbol{R}^{T}$. Considere o estimador não tendencioso de $\sigma^{2}$

$$
\hat{\sigma}^{2}=\frac{1}{n-p}(\boldsymbol{y}-\boldsymbol{X} \hat{\boldsymbol{\beta}})^{T} \hat{\Omega}^{-1}(\boldsymbol{y}-\boldsymbol{X} \hat{\boldsymbol{\beta}}),
$$

cuja distribuição não depende de $\hat{\boldsymbol{\beta}}$ e é conhecido que $(n-p) \hat{\sigma}^{2} / \sigma \sim \chi_{n-p}^{2}$. Nesse caso a estatística da razão de verossimilhança pode ser escrita como

$$
\xi_{r v}=\frac{1}{\hat{\sigma}^{2}} \tilde{\boldsymbol{\mu}}^{T} \mathbf{V}^{-1} \tilde{\boldsymbol{\mu}}
$$

Do Teorema $2.2, \tilde{\mu}^{T} \mathrm{~V}^{-1} \tilde{\mu}$ é uma mistura de variáveis aleatórias qui-quadrados centrais, as quais são independentes de $\hat{\sigma}^{2}$ e note que

$$
\frac{\hat{\sigma}^{2}}{\sigma^{2}}=\frac{(n-p) \hat{\sigma}^{2} / \sigma^{2}}{n-p} \sim \frac{\chi_{n-p}^{2}}{n-p} .
$$

Por definição, o quociente entre duas variáveis aleatórias qui-quadrados centrais independentes divididas pelos seus respectivos graus de liberdade, tem distribuição $F$ de Snedecor com graus de liberdade do numerador e do denominador iguais aos das respectivas qui-quadrados. Com isso, podemos propor como um corolário do Teorema 2.2 o seguinte corolário:

Corolário 2.1. Considere o caso de $\sigma^{2}$ desconhecido mas, existe um estimador não tendencioso $\hat{\sigma}^{2}$, de $\sigma^{2}$, cuja distribuição é independente de $\hat{\boldsymbol{\mu}}$ tal que $(n-p) \hat{\sigma}^{2} / \sigma \sim \chi_{n-p}^{2}$. 
Sob $H_{0}: \mu=0$, a distribuição de $\xi_{r v}$ para todo $c>0$ é dada por

$$
\begin{aligned}
& P\left(\xi_{r v} \geq c\right)=\sum_{\ell=1}^{k} w(k, \ell ; \mathrm{V}) P\left(F_{\ell, n-p} \geq \frac{c}{\ell}\right) \mathrm{e} \\
& P\left(\xi_{r v}=0\right)=w(k, 0 ; \mathrm{V}) .
\end{aligned}
$$

Suponha agora que $\Sigma=\sigma^{2} \Omega$ com $\sigma^{2}$ desconhecida e $\Omega$ desconhecida e positiva definida. Nesse caso, para testar as hipóteses (2.4.1), Gouriéroux et al. (1982) mostram que sendo $\left(\bar{\sigma}^{2}, \bar{\Omega}\right)$ e $\left(\tilde{\sigma}^{2}, \tilde{\Omega}\right)$ estimadores consistentes de $\left(\sigma^{2}, \Omega\right)$ sob $H_{0}$ e $H_{1}$, respectivamente, as estatísticas $\xi_{r v}, \xi_{w}$ e $\xi_{k t}$ são assintoticamente equivalentes e têm a mesma distribuição nula assintótica da estatística $\xi_{r v}$ quando $\Sigma$ é conhecida e para todo $c>0$ é dada por

$$
\begin{aligned}
& \lim _{n \rightarrow \infty} P\left(\xi_{r v} \geq c\right)=\sum_{\ell=1}^{k} w(k, \ell ; \mathrm{V}) P\left(\chi_{\ell}^{2} \geq c\right) \mathrm{e} \\
& \lim _{n \rightarrow \infty} P\left(\xi_{r v}=0\right)=w(k, 0 ; \mathrm{V}) .
\end{aligned}
$$

\section{Caso II}

Para o caso de testar as hipóteses

$$
H_{1}: \boldsymbol{\mu} \geq 0 \text { versus } H_{2}-H_{1}
$$

onde $H_{2}: \boldsymbol{\mu} \in \mathbb{R}^{k}$, para o modelo $\hat{\boldsymbol{\mu}}=\boldsymbol{\mu}+\boldsymbol{v}$, onde $\boldsymbol{\mu}=\boldsymbol{R} \boldsymbol{\beta}-\boldsymbol{r}$, com $\boldsymbol{v} \sim \mathrm{N}_{k}\left(\mathbf{0}, \sigma^{2} \mathrm{~V}\right)$, $\sigma^{2}$ desconhecido e $\mathrm{V}$ conhecida, positiva definida e dada por $\mathrm{V}=\boldsymbol{R}\left(\boldsymbol{X}^{T} \boldsymbol{\Omega}^{-1} \boldsymbol{X}\right)^{-1} \boldsymbol{R}^{T}$, a estatística da razão de verossimilhança é dada por

$$
\xi_{r v}=\frac{1}{\hat{\sigma}^{2}}(\hat{\boldsymbol{\mu}}-\tilde{\boldsymbol{\mu}})^{T} \mathbf{V}^{-1}(\hat{\boldsymbol{\mu}}-\tilde{\boldsymbol{\mu}}) .
$$

Por argumentos similares àqueles usados no Corolário 2.1, podemos propor como um corolário do Teorema 2.3 o seguinte corolário:

Corolário 2.2. Considere o caso de $\sigma^{2}$ desconhecido mas, existe um estimador não tendencioso $\hat{\sigma}^{2}$, de $\sigma^{2}$, cuja distribuição é independente de $\hat{\mu}$ tal que $(n-p) \hat{\sigma}^{2} / \sigma \sim \chi_{n-p}^{2}$. 
Sob $H_{1}: \mu \geq 0$, a distribuição de $\xi_{\text {rv }}$ para todo $c>0$ é dada por

$$
\begin{aligned}
\sup _{\boldsymbol{\mu} \geq 0} P\left(\xi_{r v} \geq c\right) & =P\left(\xi_{r v} \geq c \mid \boldsymbol{\mu}=0\right) \\
= & \sum_{\ell=1}^{k} w(k, k-\ell ; \mathrm{V}) P\left(F_{\ell, n-p} \geq \frac{c}{k-\ell}\right) \mathrm{e} \\
\sup _{\boldsymbol{\mu} \geq \mathbf{0}} P\left(\xi_{r v}=0\right) & =P\left(\xi_{r v}=0 \mid \boldsymbol{\mu}=0\right) \\
& =w(k, k ; \mathrm{V}) .
\end{aligned}
$$

Suponha agora que $\Sigma=\sigma^{2} \Omega$ com $\sigma^{2}$ desconhecida e $\Omega$ desconhecida e positiva definida. Nesse caso, para testar as hipóteses (2.4.2), Wolak (1989) mostra que sendo $\left(\tilde{\sigma}^{2}, \tilde{\Omega}\right)$ e $\left(\hat{\sigma}^{2}, \hat{\Omega}\right)$ estimadores consistentes de $\left(\sigma^{2}, \Omega\right)$ sob $H_{1}$ e $H_{2}$, respectivamente, as estatísticas $\xi_{r v}, \xi_{w}$ e $\xi_{k t}$ são assintoticamente equivalentes e têm a mesma distribuição nula assintótica da estatística $\xi_{r v}$ quando $\Sigma$ é conhecida. A distribuição nula menos farorável é alcançada em $\boldsymbol{R} \boldsymbol{\beta}=\boldsymbol{r}$ e para todo $c>0$ é dada por

$$
\begin{aligned}
\lim _{n \rightarrow \infty} P\left(\xi_{r v} \geq c\right) & =\sum_{\ell=1}^{k} w(k, k-\ell ; \mathrm{V}) P\left(\chi_{\ell}^{2} \geq c\right) \mathrm{e} \\
\lim _{n \rightarrow \infty} P\left(\xi_{r v}=0\right) & =w(k, k ; \mathrm{V}) .
\end{aligned}
$$

\subsection{Aplicações}

\subsubsection{Pré-teste pós-teste}

A Tabela 2.7.1, obtida de Moore e McCabe (1993), apresenta os escores obtidos por 20 professores de $2^{\underline{O}}$ grau antes e depois de um curso intensivo de Espanhol. É bastante razoável esperar que o curso intensivo aumente ou pelo menos não diminua a habilidade dos professores em Espanhol. Portanto, sendo $\left(x_{i}, y_{i}\right)$ o par de valores do pré-teste e pós-teste para o $i$-ésimo professor, $i=1,2, \ldots, 20$, vamos assumir o modelo dado em 
(2.3.7), isto é, $y_{i}=\beta x_{i}+\epsilon_{i}$ sujeito a $\beta \geq 1$ com $\epsilon_{i} \sim \mathrm{N}\left(0, \sigma^{2}\right)$ independentes e verificar se o curso intensivo aumenta a habilidade dos professores em Espanhol através das hipóteses

$$
H_{0}: \beta=1 \text { versus } H_{1}: \beta>1
$$

Tabela 2.7.1: Escores de um pré-teste pós-teste realizado em Espanhol para 20 professores.

\begin{tabular}{ccc|ccc}
\hline Professor & Pré-teste & Pós-teste & Professor & Pré-teste & Pós-teste \\
\hline \hline 1 & 30 & 29 & 11 & 30 & 32 \\
2 & 28 & 30 & 12 & 29 & 28 \\
3 & 31 & 32 & 13 & 31 & 34 \\
4 & 26 & 30 & 14 & 29 & 32 \\
5 & 20 & 16 & 15 & 34 & 32 \\
6 & 30 & 25 & 16 & 20 & 27 \\
7 & 34 & 31 & 17 & 26 & 28 \\
8 & 15 & 18 & 18 & 25 & 29 \\
9 & 28 & 33 & 19 & 31 & 32 \\
10 & 20 & 25 & 20 & 29 & 32 \\
\hline
\end{tabular}

A estimativa não restrita de $\beta$ fica dada por $\hat{\beta}=1,0433(0,0267)$. Uma vez que $\hat{\beta}>1$ a estimativa restrita (sob $H_{1}$ ) fica também dada por $\tilde{\beta}=1,0433$. A estatística da razão de verossimilhança $(2.6 .16)$, com $\tilde{\mu}=\tilde{\beta}-1$, fica nesse caso dada por

$$
\xi_{r v}=\frac{1}{\hat{\sigma}^{2}} \tilde{\mu}^{T} \mathrm{~V}^{-1} \tilde{\boldsymbol{\mu}}=\frac{1}{\hat{\sigma}^{2}}(\tilde{\beta}-1)^{2} \sum_{i=1}^{20} x_{i}^{2},
$$

onde $\hat{\sigma}^{2}$ é obtido de (2.6.15) e fica dado nesse caso por $\hat{\sigma}^{2}=\frac{1}{19} \sum_{i=1}^{20}\left(y_{i}-x_{i} \hat{\beta}\right)^{2}$. Segue 
então do Corolário 2.1 que sob $H_{0}$

$$
\begin{aligned}
P\left(\xi_{r v} \geq c \mid \boldsymbol{\mu}=0\right) & =\sum_{\ell=1}^{k} w(k \cdot \ell ; \mathbf{V}) P\left(F_{\ell, n-p} \geq \frac{c}{\ell}\right) \\
& =\frac{1}{2} P\left(F_{1.19} \geq c\right)
\end{aligned}
$$

uma vez que $k=1$ e $w(1,0 ; \mathrm{V})=w(1,1 ; \mathrm{V})=1 / 2$. Foi obtido o valor $\xi_{r v}=2,63$ cujo nível descritivo é dado por $P=0,0607$, que é significativo a $10 \%$. Se fosse aplicado o teste bicaudal, $H_{0}: \beta=1$ versus $H_{1}^{*}: \beta \neq 1$, a estatística da razão de verossimilhança que também nesse caso é dada por (2.7.1) e sob $H_{0}$ segue uma distribuição $\chi_{1}^{2}$ levaria ao nível descritivo $P=0,1213$, que não é significativo a $10 \%$.

\subsubsection{Assinaturas de TV a cabo}

No Apêndice D é apresentado um conjunto de dados sobre a demanda de TVs a cabo em 40 áreas metropolitanas (Ramanathan, 1993). Foram observadas, para cada região, o número de assinantes (em milhares) de TV a cabo (Nass), o número de domicílios (em milhares) na área (Domic), a renda per capita (em US\$) por domicílio com TV a cabo (Percap), a taxa de instalação (Taxa). o custo médio mensal de manutenção (Custo), o número de canais a cabo disponíveis na área (Ncabo) e o número de canais não pagos com sinal de boa qualidade disponíveis na área (Ntv). Ramanathan propõe, para explicar a variável resposta Nass, o modelo dado abaixo

$$
\begin{aligned}
\text { Nass }_{i}= & \beta_{1}+\beta_{2} \text { Domic }_{i}+\beta_{3} \text { Percap }_{i}+\beta_{4} \text { Taxa }_{i} \\
& +\beta_{5} \text { Custo }_{i}+\beta_{6} \text { Ncabo }_{i}+\beta_{7} \text { Ntv }_{i}+\varepsilon_{i}
\end{aligned}
$$

onde $\varepsilon_{i} \sim N\left(0, \sigma^{2}\right)$ são erros mutuamente independentes, $i=1, \ldots, 40$. Justifica-se a seguir a imposição de restrições nos parâmetros do modelo. Diferenciando, por exemplo, parcialmente a equação $(2.7 .2)$ com respeito a Custo, obtém-se $\beta_{5}=\partial \mathrm{Nass} / \partial \mathrm{Custo}$. 
Isso significa que, mantendo-se as demais variáveis explicativas fixas, se o custo médio mensal de manutenção é acrescido de US\$ 1, então o número de assinantes deverá variar, em média, por $\beta_{5}$. Espera-se, nesse caso, que o número de assinantes decresça à medida que o custo de manutenção aumente. Isso implica na imposição de $\beta_{5} \leq 0$. Aplicando-se esse mesmo raciocínio para as covariáveis restantes, chega-se às restrições adicionais $\beta_{2} \geq 0, \beta_{3} \geq 0, \beta_{4} \leq 0, \beta_{6} \geq 0$ e $\beta_{7} \leq 0$. A Tabela 2.7 .2 apresenta as estimativas não restritas dos parâmetros com seus respectivos desvios padrão, bem como os níveis descritivos correspondentes ao teste $t$ unilateral para testar $H_{0}: \beta_{i}=0$ versus $H_{1}: \beta_{i}>0, i=2, \ldots, 7$. Nota-se que para um nível de significância de $5 \%$, apenas os coeficientes de Domic e Ntv são significativamente maiores que zero.

Tabela 2.7.2: Estimativas não restritas com o respectivo desvio padrão dos parâmetros do modelo para explicar o número de assinantes de TV a cabo.

\begin{tabular}{lrrrr}
\hline Variável & $\hat{\beta}$ & $\operatorname{Dp}(\hat{\beta})$ & $t_{c}$ & $\mathrm{P}$ \\
\hline \hline Constante & $-28,9612$ & 26,6712 & $-1,0859$ & 0.1422 \\
Domic & 0,4384 & 0,0343 & 12,7636 & $<0,0001$ \\
Percap & 0,0055 & 0,0032 & 1,6622 & 0,0524 \\
Taxa & $-0,1596$ & 0,4809 & $-0,3318$ & 0,3709 \\
Custo & 0,6092 & 2,1786 & 0,2796 & 0,3907 \\
Ncabo & 1,1299 & 0,7151 & 1,5800 & 0,0612 \\
Ntv & $-5,6965$ & 1,6001 & $-3,5600$ & 0,0005 \\
\hline
\end{tabular}

Pode-se verificar também qual é o nível descritivo associado quando testamos conjuntamente se os quatro coeficientes eliminados, Percap, Taxa, Custo e Ncabo são iguais a zero ou se há pelo menos um maior que zero. As hipóteses, de acordo com as restrições 
impostas aos parâmetros ficam, nesse caso, dadas por $H_{0}: \boldsymbol{R} \boldsymbol{\beta}=\mathbf{0}$ versus $H_{1}: \boldsymbol{R} \boldsymbol{\beta} \geq \mathbf{0}$, com pelo menos uma desigualdade estrita em $H_{1}$, onde

$$
\boldsymbol{R}=\left(\begin{array}{rrrrrrr}
0 & 0 & 1 & 0 & 0 & 0 & 0 \\
0 & 0 & 0 & -1 & 0 & 0 & 0 \\
0 & 0 & 0 & 0 & -1 & 0 & 0 \\
0 & 0 & 0 & 0 & 0 & 1 & 0
\end{array}\right)
$$

e $\boldsymbol{\beta}=\left(\beta_{1}, \beta_{2}, \ldots, \beta_{7}\right)^{T}$. A estatística da razão de verossimilhança (2.6.16) fica, nesse caso, dada por

$$
\xi_{r v}=\frac{(\boldsymbol{R} \tilde{\boldsymbol{\beta}})^{T} \mathbf{V}^{-1}(\boldsymbol{R} \tilde{\boldsymbol{\beta}})}{\hat{\sigma}^{2}}=\frac{994,8886}{175,50}=5,6689,
$$

onde $\mathrm{V}=\boldsymbol{R}\left(\boldsymbol{X}^{T} \boldsymbol{X}\right)^{-1} \boldsymbol{R}^{T}$ e $\hat{\sigma}^{2}$ é dado por (2.6.15). Como há apenas uma violação entre as estimativas não restritas, a qual corresponde à variável Custo $\left(\hat{\beta}_{5}>0\right)$, as estimativas restritas dos coeficientes são obtidas recalculando-se as estimativas não restritas do modelo (2.7.2) sem o coeficiente de Custo $\left(\beta_{5}=0\right)$. Pelo algoritmo dado na Seção 2.3 tem-se que $\tilde{\boldsymbol{\beta}}=(-26,4512,0,4403,0,0055,-0,1285,0,0000,1,1699,-5.6512)^{T}$. Pelo Corolário 2.1 e as expressões para os pesos apresentadas no Apêndice A, o nível descritivo do teste é dado por

$$
\begin{aligned}
P & =\sum_{\ell=1}^{4} w(k, \ell ; \mathrm{V}) P\left(F_{\ell,(n-p)} \geq \frac{5,6689}{\ell}\right) \\
& =0,2390 \times P\left(F_{1,33} \geq 5,6689\right)+0,3736 \times P\left(F_{2,33} \geq 2,8345\right) \\
& +0,2610 \times P\left(F_{3,33} \geq 1,8896\right)+0,0661 \times P\left(F_{4,33} \geq 1,4172\right) \\
& =0,00554+0,02731+0,03929+0,01654 \\
& =0,08869 .
\end{aligned}
$$

Portanto, ao nível de $5 \%$ de significância, não rejeitamos $H_{0}$ e ficamos com o modelo apenas com as variáveis explicativas Domic e Ntv. Diagnósticos para avaliar a adequação desse modelo ou mesmo a eliminação dos demais coeficientes podem ser aplicados usando, por exemplo, os métodos propostos por Paula (1993). 


\subsubsection{Demanda de energia elétrica}

Como uma aplicação das estatísticas para testar as hipóteses (2.4.2), vamos considerar o problema de teste de hipóteses na análise de uma equação de demanda (Wolak, 1989). Considere a seguinte equação de demanda:

$$
\begin{aligned}
\ln \left(Q_{t}\right) & =\alpha+\beta_{1} \ln \left(P E_{t}\right)+\beta_{2} \ln \left(P G_{t}\right)+\beta_{3} \ln \left(Y_{t}\right) \\
& +\beta_{4} D 1_{t}+\beta_{5} D 2_{t}+\beta_{6} D 3_{t}+\varepsilon_{t},
\end{aligned}
$$

onde $\varepsilon_{t}=\rho \varepsilon_{t-1}+\eta_{t}$ e $\eta_{t} \sim \mathrm{N}\left(0, \sigma^{2}\right)$. A equação (2.7.3) pode ser escrita na forma matricial como $\boldsymbol{y}=\boldsymbol{X} \boldsymbol{\beta}+\boldsymbol{\varepsilon}$, com $\varepsilon \sim \mathrm{N}_{n}\left\{\mathbf{0}, \sigma^{2} \mathrm{~V}(\rho)\right\}$, onde $\mathrm{V}(\rho)$ é definido como em Theil $\left(1971\right.$, p. 252). As variáveis $Q_{t}, P E_{t}, P G_{t}$ e $Y_{t}$ representam, respectivamente, a demanda de eletricidade agregada per capita para o setor residencial, o preço médio da eletricidade para o setor residencial, o preço do gás natural para o setor residencial e a renda per capita. $D 1_{t}, D 2_{t}$ e $D 3_{t}$ são variáveis binárias e foram incluídas porque os dados estão em bases trimestrais. $t$ representa o trimestre e os dados foram coletados desde o primeiro trimestre de 1961 até o quarto trimestre de 1983, num total de 92 observações. O conjunto de dados usado nesse exemplo é apresentado no Apêndice D.

É razoável esperar que a demanda de eletricidade cresça quando diminui o preço da eletricidade e aumenta o preço do gás natural e a renda per capita, portanto, é de interesse testar inicialmente se o subvetor $\left(-\beta_{1}, \beta_{2}, \beta_{3}\right)^{T}$ é maior ou igual a zero. Vamos testar então as hipóteses $H_{1}: \boldsymbol{R} \boldsymbol{\beta} \geq \boldsymbol{r}$ versus $H_{2}-H_{1}$, onde $H_{2}: \boldsymbol{\beta} \in \mathbb{R}^{7}$, com

$$
\boldsymbol{R}=\left(\begin{array}{ccccccc}
0 & -1 & 0 & 0 & 0 & 0 & 0 \\
0 & 0 & 1 & 0 & 0 & 0 & 0 \\
0 & 0 & 0 & 1 & 0 & 0 & 0
\end{array}\right), \quad \boldsymbol{r}=\left(\begin{array}{l}
0 \\
0 \\
0
\end{array}\right) \text { e } \boldsymbol{\beta}=\left(\alpha, \beta_{1}, \beta_{2}, \beta_{3}, \beta_{4}, \beta_{5}, \beta_{6}\right)^{T}
$$

A Tabela 2.7.3 apresenta as estimativas não restritas e as estimativas restritas com os respectivos desvios padrão para os parâmetros do modelo (2.7.3). Como ocorreu 
somente uma violação entre as estimativas não restritas $\left(\hat{\beta}_{2}<0\right)$, as estimativas restritas foram obtidas calculando-se as estimativas não restritas no modelo (2.7.3) com $\beta_{5}=$ 0, de acordo com o procedimento descrito na Seção 2.3. Esse procedimento produz estimativas consistentes de $\sigma^{2}$ e $\rho$ as quais foram usadas para determinar uma estimativa consistente de $\sigma^{2} \mathrm{~V}(\rho)$, que é necessária para calcular as estatísticas dos testes.

Tabela 2.7.3: Estimativas dos parâmetros do modelo para explicar a demanda de eletricidade agregada per capita.

\begin{tabular}{lcccccccc}
\hline Coeficiente & $\alpha$ & $\beta_{1}$ & $\beta_{2}$ & $\beta_{3}$ & $\beta_{4}$ & $\beta_{5}$ & $\beta_{6}$ & $\rho$ \\
\hline \hline \multirow{8}{c}{ Estimativas não restritas } \\
Estimativa & $-0,918$ & $-0,115$ & $-0,179$ & 1,811 & 0,113 & $-0,048$ & 0,143 & 0,667 \\
Desvio padrão & $0,68 j$ & 0,127 & 0,079 & 0,090 & 0,012 & 0,012 & 0,018 & 0,082 \\
& \multicolumn{8}{c}{ Estimativas restritas } \\
Estimativa & 0,205 & $-0,289$ & 0,000 & 1,666 & 0,121 & $-0,049$ & 0,109 & 0,653 \\
Desvio padrão & 0,464 & 0,102 & 0,000 & 0,062 & 0,012 & 0,012 & 0,011 & 0,082 \\
\hline
\end{tabular}

As estatística $\xi_{r v}, \xi_{w}$ e $\xi_{k t}$ foram calculadas de (2.6.10), (2.6.11) e (2.6.13), respectivamente, e os valores obtidos foram $\xi_{w}=6,321, \xi_{r v}=5,581$ e $\xi_{k t}=4,448$, os quais estão de acordo com a relação apresentada na Seção 2.6.

Os pesos usados na distribuição nula das estatísticas dos testes foram estimados usando os coeficientes de correlação e correlação parcial obtidos da matriz estimada de covariância para as estimativas dos parâmetros do modelo não restrito, dada por $\hat{\mathrm{V}}=$ $\boldsymbol{R}\left(\boldsymbol{X}^{T} \hat{\boldsymbol{\Sigma}}^{-1} \boldsymbol{X}\right)^{-1} \boldsymbol{R}^{T}$, onde $\hat{\boldsymbol{\Sigma}}=\hat{\sigma}^{2} \mathbf{V}(\hat{\rho})$. 
Os níveis descritivos dos testes podem ser calculados de (2.6.19) sendo dados por

$$
P\left(\xi^{*} \geq c\right)=\sum_{\ell=1}^{3} w(3,3-\ell ; \hat{\mathbf{V}}) P\left(\chi_{\ell}^{2} \geq c\right),
$$

onde $\xi^{*}$ representa $\xi_{w}, \xi_{r v}$ ou $\xi_{k t}$ e $c$ é o correspondente valor obtido para cada uma das estatísticas dos testes. A Tabela 2.7.4 apresenta os pesos estimados, os níveis descritivos e o valor de $P\left(\chi_{\ell}^{2} \geq c\right)$ para cada uma das estatísticas e níveis descritivos das estatísticas dos testes.

Tabela 2.7.4: Pesos e níveis descritivos das estatísticas dos testes.

\begin{tabular}{ccccc}
\multicolumn{5}{c}{ Pesos estimados e probabilidades $P\left(\chi_{\ell}^{2} \geq c\right)$} \\
\hline \hline$w(3, \ell ; \hat{\mathrm{V}})$ & 0,153 & 0,432 & $\ell=2$ & $\ell=3$ \\
$P\left(\chi_{\ell}^{2} \geq c\right)$ & 0,000 & 0,012 & 0,347 & 0,068 \\
$P\left(\chi_{\ell}^{2} \geq c\right)$ & 0,000 & 0,018 & 0,061 & 0,097 \\
$P\left(\chi_{\ell}^{2} \geq c\right)$ & 0,000 & 0,035 & 0,108 & 0,134 \\
\hline
\end{tabular}

Níveis descritivos

\begin{tabular}{ccc}
\hline Estatística & $c$ & $P=P\left(\xi^{*} \geq c\right)$ \\
\hline \hline$\xi_{w}$ & 6,321 & 0,037 \\
$\xi_{r v}$ & 5,581 & 0,053 \\
$\xi_{k t}$ & 4,448 & 0,092 \\
\hline
\end{tabular}

Verifica-se que ao nível de $5 \%$ de significância, a hipótese nula não pode ser rejeitada pelas estatísticas $\xi_{k t}$ e $\xi_{r v}$. Como descrito por Wolak (1989), dado que não rejeita-se a hipótese nula do teste de $H_{1}: \boldsymbol{R} \boldsymbol{\beta} \geq \boldsymbol{r}$ versus $H_{2}-H_{1}$, onde $H_{2}: \boldsymbol{\beta} \in \mathbb{R}^{7}$, pode-se pensar em testar as hipóteses $H_{0}: \boldsymbol{R} \boldsymbol{\beta}=\boldsymbol{r}$ versus $\quad H_{1}: \boldsymbol{R} \boldsymbol{\beta} \geq \boldsymbol{r}$ com pelo menos uma 
desigualdade estrita. Se a hipótese nula desse teste for rejeitada em favor da alternativa restrita, esse resultado combinado com o resultado do primeiro teste é uma evidência de que a hipótese de restrição de desigualdade vale estritamente para pelo menos uma componente do subvetor de parâmetros sob investigação. 


\section{Capítulo 3}

\section{Equações de Estimação}

\section{Generalizadas}

\subsection{Introdução}

Dados longitudinais com variável resposta contínua, em geral são analisados através de modelos lineares com erros correlacionados. Quando a variável resposta é aproximadamente normal, existem várias técnicas estatísticas bem desenvolvidas (veja, por exemplo, Laird e Ware, 1982 e Ware, 1985). Para variável resposta binária, modelos de medidas repetidas em que a correlação entre as observações da mesma unidade amostral é constante para todo par de observações foram propostos por Ochi e Prentice (1984) usando a ligação probit, Koch et al. (1977) usando modelos log-lineares e Stiratelli et al. (1984) usando a ligação logit. Zeger et al. (1985) apresentam um modelo de cadeias de Markov de primeira ordem para dados longitudinais. 
Uma dificuldade na análise de dados longitudinais com resposta não normal é a falta de uma classe rica de modelos como a normal multivariada para a distribuição conjunta das observações. Liang e Zeger (1986) apresentam uma extensão dos modelos lineares generalizados para a análise de dados longitudinais, introduzindo uma nova classe de equações de estimação, a qual denominaram Equações de Estimação Generalizadas EEGs, que produzem estimadores consistentes para os parâmetros da regressão e para a variância desses estimadores sob suaves condições de regularidade. O principal fundamento das EEGs é usar um modelo linear generalizado para a distribuição marginal de cada observação, sem especificar a forma da distribuição conjunta das medidas repetidas, porém especificando a estrutura de correlação entre as observações. O método se reduz ao de máxima verossimilhança quando a variável resposta é normal multivariada.

\subsection{Modelos lineares generalizados}

Os modelos lineares generalizados desempenham hoje um papel muito importante na Estatística, provavelmente o mesmo papel da regressão normal linear na década de 60 . Essa classe de modelos proposta por Nelder e Wedderburn (1972), generaliza o modelo tradicional de regressão normal linear, oferecendo várias opções para a distribuição da variável resposta e dando maior flexibilidade para a ligação entre a média da variável resposta e a parte sistemática do modelo. A suposição de distribuição normal para a variável resposta é substituída pela suposição de que a variável resposta pertence à família exponencial de distribuições. A ligação entre a média e o preditor linear não é mais necessariamente a identidade, podendo assumir qualquer forma monótona não linear. O processo iterativo para a estimação dos parâmetros do modelo, pode ser visto como um método de mínimos quadrados reponderados. Enfim, toda a estrutura 
conhecida para a regressão normal linear, pode ser estendida para os modelos lineares generalizados. A grande vantagem disso, é a possibilidade do estudo conjunto das propriedades de diferentes modelos de regressão, entre os quais podemos destacar o modelo de regressão logística e os modelos log-lineares, embora cada modelo tenha propriedades intrínsecas que devem ser estudadas separadamente.

Muitas extensões dos modelos lineares generalizados surgiram nos últimos 25 anos. A principal delas, proposta por Wedderburn (1974), são os modelos de quase-verossimilhança. Os modelos de dispersão (Jørgensen, 1983) ampliam o leque de opções para a distribuição da variável resposta. Os modelos não lineares de familia exponencial (Cordeiro e Paula, 1989) admitem preditores não lineares nos parâmetros. Os modelos aditivos generalizados (Hastie e Tibshirani, 1990) permitem que o preditor seja também formado por funções semi-paramétricas. Temos ainda os modelos lineares generalizados mistos (Breslow e Clayton, 1993) que admitem a inclusão de efeitos aleatórios normais no preditor linear. Recentemente, Lee e Nelder (1996) estenderam o trabalho de Breslow e Clayton (1993) propondo modelos lineares generalizados hierárquicos em que o preditor linear pode ser formado por efeitos fixos e efeitos aleatórios não normais. Muitas outras aplicações da estrutura dos modelos lineares generalizados podem ser encontradas em diversos artigos e livros da literatura Estatística. A principal referência no assunto é o livro de McCullagh e Nelder (1989).

Nas Seções 3.2.1 a 3.2.5 apresentaremos uma revisão dos principais resultados relacionados com os modelos lineares generalizados. Para aqueles leitores familiarizados com esses conceitos a leitura dessas seções pode ser omitida indo-se diretamente para a Seção 3.2.6. 


\subsubsection{Definição}

Sejam $y_{1}, y_{2}, \ldots, y_{n}$ variáveis aleatórias independentes, cada uma com distribuição pertencente à família exponencial, com densidade (ou função de probabilidade) dada por

$$
f\left(y ; \theta_{i}, \phi\right)=\exp \left\{\phi\left[y \theta_{i}-b\left(\theta_{i}\right)\right]+c(y, \phi)\right\}
$$

onde $b(\cdot)$ e $c(\cdot)$ são funções conhecidas, $\mathrm{E}\left(y_{i}\right)=\mu_{i}=b^{\prime}\left(\theta_{i}\right), \operatorname{Var}\left(y_{i}\right)=\phi^{-1} V_{i}, V=$ $d \mu / d \theta$ é a função de variância e $\phi^{-1}>0$ é o parâmetro de dispersão. A função de variância desempenha um importante papel na família exponencial, uma vez que a mesma caracteriza a distribuição. Isto é, dada a função de variância, tem-se uma classe de distribuições correspondentes e vice-versa. Essa é uma propriedade importante que permite a comparação de distribuições através de testes para a função de variância. Por exemplo, a função de variância definida por $V(\mu)=\mu(1-\mu)$, caracteriza a classe de distribuições binomiais com probabilidade de sucesso $\mu$ ou $(1-\mu)$ (veja, por exemplo, Jørgensen, 1996). Os modelos lineares generalizados são definidos por (3.2.1) e pela componente sistemática

$$
g\left(\mu_{i}\right)=\eta_{i}
$$

onde $\eta_{i}=\boldsymbol{x}_{i}^{T} \boldsymbol{\beta}$ é o preditor linear, $\boldsymbol{\beta}=\left(\beta_{1}, \beta_{2}, \ldots, \beta_{p}\right)^{T}, p<n$, é um vetor de parâmetros desconhecidos a serem estimados dos dados, $\boldsymbol{x}_{i}=\left(x_{i 1}, x_{i 2}, \ldots, x_{i p}\right)^{T}$ representa os valores de $p$ variáveis explicativas e $g(\cdot)$ é uma função monótona e diferenciável, denominada função de ligação. Apresentaremos a seguir as distribuições mais conhecidas pertencentes à família exponencial. 


\subsubsection{Casos particulares}

\section{Normal}

Se a variável aleatória $y$ tem uma distribuição normal com média $\mu$ e variância $\sigma^{2}$, isto é, $y \sim \mathrm{N}\left(\mu, \sigma^{2}\right)$, então sua densidade pode ser escrita como

$$
f\left(y ; \mu, \sigma^{2}\right)=\exp \left\{\frac{1}{\sigma^{2}}\left[y \mu-\frac{\mu^{2}}{2}\right]-\frac{1}{2}\left[\frac{y^{2}}{\sigma^{2}}+\ln \left(2 \pi \sigma^{2}\right)\right]\right\},
$$

onde $-\infty<y, \mu<\infty$ e $\sigma^{2}>0$. Fazendo $\phi^{-1}=\sigma^{2}, \theta=\mu, b(\theta)=\frac{1}{2} \theta^{2}$, e $c(y, \phi)=$ $-\frac{1}{2}\left\{\frac{y^{2}}{\sigma^{2}}+\ln \left(2 \pi \sigma^{2}\right)\right\}$, tem-se a forma (3.2.1). A função de variância da normal é dada por $V(\mu)=1$.

\section{Poisson}

Se $y$ tem distribuição de Poisson com parâmetro $\mu$, isto é, $y \sim P(\mu)$, onde $\mu>0$ e $y \in\{0,1,2, \ldots\}$, então a função de probabilidade de $y$ pode ser ecrita na forma

$$
f(y ; \mu)=\exp \{[y \ln (\mu)-\mu]-\ln (y !)\}
$$

Com $\phi^{-1}=1, \theta=\ln (\mu), b(\theta)=e^{\theta}$ e $c(y, \phi)=-\ln (y !)$, tem-se (3.2.1). A função de variância da Poisson é dada por $V(\mu)=\mu$.

\section{Binomial}

Seja $y$ a proporção de sucessos em $n$ ensaios de Bernoulli com probabilidade de sucesso igual a $\mu$. Assumindo que ny tem distribuição binomial com parâmetros $n$ e $\mu$, isto é, $n y \sim B(n, \mu)$, onde $n$ é um inteiro maior ou igual a $1,0<\mu<1$ e $y \in(0,1)$, então a função de probabilidade de $y$ pode ser escrita na forma

$$
f(y ; \mu)=\exp \left\{n\left[y \ln \left(\frac{\mu}{1-\mu}\right)+\ln (1-\mu)\right]+\ln \left(\begin{array}{c}
n \\
n y
\end{array}\right)\right\} .
$$

A forma (3.2.1) é obtida fazendo-se $\phi^{-1}=\frac{1}{n}, \theta=\ln \left\{\frac{\mu}{1-\mu}\right\}, b(\theta)=\ln \left(1+e^{\theta}\right)$ e $c(y, \phi)=$ $\ln \left(\begin{array}{c}\phi \\ \phi y\end{array}\right)$. A função de variância da binomial é dada por $V(\mu)=\mu(1-\mu)$. 


\section{Gama}

Seja y uma variável aleatória com distribuição gama de média $\mu$ e coeficiente de variação $\phi^{-1 / 2}$, denotaremos $y \sim G(\mu, \phi)$. A densidade de $y$ pode ser escrita na forma

$$
f(y ; \mu, \phi)=\exp \left\{\phi\left[-\frac{y}{\mu}+\ln \left(\frac{1}{\mu}\right)\right]-\ln \Gamma(\phi)+\phi \ln (\phi y)-\ln (y)\right\}
$$

onde $y \geq 0, \phi>0$ e $\mu>0$. Portanto, fazendo $\theta=-\frac{1}{\mu}, b(\theta)=-\ln (-\theta)$ e $c(y, \phi)=$ $(\phi-1) \ln (y)+\phi \ln (\phi)-\ln \Gamma(\phi)$, tem-se (3.2.1). A função de variância da gama é dada por $V(\mu)=\mu^{2}$.

\section{Normal inversa}

Seja $y$ uma variável aleatória com distribuição normal inversa de média $\mu$ e parâmetro de forma $\sigma^{2}$, isto é, $y \sim \mathrm{NI}\left(\mu, \sigma^{2}\right)$, onde $y \in(0, \infty), \mu>0$ e $\sigma^{2}>0$. A densidade de $y$ pode ser escrita como

$$
f\left(y ; \mu, \sigma^{2}\right)=\exp \left\{\frac{1}{\sigma^{2}}\left[-\frac{y}{2 \mu^{2}}+\frac{1}{\mu}\right]-\frac{1}{2} \ln \left(2 \pi \sigma^{2} y^{3}\right)-\frac{1}{2 \sigma^{2} y}\right\} .
$$

$\operatorname{Com} \phi^{-1}=\sigma^{2}, \theta=\left(-2 \mu^{2}\right)^{-1 / 2}, b(\theta)=-(-2 \theta)^{1 / 2}$ e $c(y, \phi)=-\frac{1}{2} \ln \left(\frac{2 \pi y^{3}}{\phi}\right)-\frac{\phi}{2 y}$, tem-se (3.2.1). A função de variância da normal inversa é dada por $V(\mu)=\mu^{3}$.

\subsubsection{Estatísticas suficientes e ligações canônicas}

Para um modelo linear generalizado com respostas independentes, o logaritmo da função de verossimilhança pode ser expresso na forma

$$
L(\boldsymbol{\beta} ; \boldsymbol{y})=\sum_{i=1}^{n} \phi\left\{y_{i} \theta_{i}-b\left(\theta_{i}\right)\right\}+\sum_{i=1}^{n} c\left(y_{i}, \phi\right) .
$$

Um caso particular importante ocorre quando o parâmetro natural da família exponencial coincide com o preditor linear, isto é, $\theta_{i}=\eta_{i}=\sum_{j=1}^{p} x_{i j} \beta_{j}$. Nesse caso, a log 
verossimilhança fica dada por

$$
L(\boldsymbol{\beta} ; \boldsymbol{y})=\sum_{i=1}^{n} \phi\left\{y_{i} \sum_{j=1}^{n} x_{i j} \beta_{j}-b\left(\sum_{j=1}^{n} x_{i j} \beta_{j}\right)\right\}+\sum_{i=1}^{n} c\left(y_{i}, \phi\right) .
$$

Definindo a estatística $S_{j}=\phi \sum_{i=1}^{n} y_{i} x_{i j}$, a log verossimilhança torna-se

$$
L(\boldsymbol{\beta} ; \boldsymbol{y})=\sum_{j=1}^{p} s_{j} \beta_{j}-\phi \sum_{i=1}^{n} b\left(\sum_{j=1}^{n} x_{i j} \beta_{j}\right)+\sum_{i=1}^{n} c\left(y_{i}, \phi\right) .
$$

Portanto, pelo teorema da fatorização a estatística $S=\left(S_{1}, S_{2}, \ldots, S_{p}\right)^{T}$ é suficiente e minimal para o vetor $\boldsymbol{\beta}=\left(\beta_{1}, \beta_{2}, \ldots, \beta_{p}\right)^{T}$. As ligações correspondentes às estatísticas suficientes são chamadas de ligações canônicas e são muito importantes para a teoria dos modelos lineares generalizados. Uma das vantagens de se usar ligações canônicas é que as mesmas garantem a concavidade da log verossimilhança e como consequência, muitos resultados assintóticos são obtidos mais facilmente. Por exemplo, a concavidade de $L(\boldsymbol{\beta} ; \boldsymbol{y})$ em $\boldsymbol{\beta}$ garante a unicidade da estimativa de máxima verossimilhança de $\boldsymbol{\beta}$, quando a mesma existe (veja, por exemplo, Paula, 1997). As ligações canônicas para os modelos mais conhecidos são dadas na Tabela 3.2.1.

\subsubsection{Função desvio}

Sem perda de generalidade, suponha agora que a log verossimilhança seja definida por

$$
L(\boldsymbol{\mu} ; \boldsymbol{y})=\sum_{i=1}^{n} L\left(\mu_{i} ; y_{i}\right)
$$

onde $\mu_{i}=g^{-1}\left(\eta_{i}\right)$ e $\eta_{i}=\boldsymbol{x}_{i}^{T} \boldsymbol{\beta}$. Para o modelo saturado $(p=n)$, a função $L(\boldsymbol{\mu} ; \boldsymbol{y})$ é estimada por

$$
L(\boldsymbol{y} ; \boldsymbol{y})=\sum_{i=1}^{n} L\left(y_{i} ; y_{i}\right)
$$


Tabela 3.2.1: Ligação canônica para os modelos mais conhecidos e outras ligações.

\begin{tabular}{ll}
\hline Modelo & Ligação canônica \\
\hline \hline Normal & $g(\mu)=\mu$ \\
Poisson & $g(\mu)=\ln (\mu)$ \\
Binomial & $g(\mu)=\ln \left\{\frac{\mu}{1-\mu}\right\}$ \\
Gama & $g(\mu)=\frac{1}{\mu}$ \\
Normal Inversa & $g(\mu)=\frac{1}{\mu^{2}}$ \\
\hline Outras ligações & \\
\hline \hline Complementar log-log & $g(\mu)=\ln \{-\ln (1-\mu)\}$ \\
Probit ${ }^{1}$ & $g(\mu)=\Phi^{-1}(\mu)$ \\
\hline & T(·) é a função de distribuição acumulada da normal \\
\multicolumn{2}{l}{ padrão. }
\end{tabular}

A estimativa de máxima verossimilhança de $\mu_{i}$ nesse caso fica dada por $\tilde{\mu}_{i}=y_{i}$. Para o modelo com $p$ parâmetros $(p<n)$, denotaremos a estimativa de $L(\boldsymbol{\mu} ; \boldsymbol{y})$ por $L(\hat{\boldsymbol{\mu}} ; \boldsymbol{y})$. Nesse caso a estimativa de máxima verossimilhançade $\mu_{i}$ será dada por $\hat{\mu}_{i}=g^{-1}\left(\hat{\eta}_{i}\right)$, onde $\hat{\eta}_{i}=\boldsymbol{x}_{i}^{T} \hat{\boldsymbol{\beta}}$. A qualidade do ajuste de um modelo linear generalizado é avaliada através da função desvio

$$
D^{*}(\boldsymbol{y} ; \hat{\boldsymbol{\mu}})=\phi D(\boldsymbol{y} ; \hat{\boldsymbol{\mu}})=2\{L(\boldsymbol{y} ; \boldsymbol{y})-L(\hat{\boldsymbol{\mu}} ; \boldsymbol{y})\}
$$

que é uma distância entre a log verossimilhança do modelo saturado ( $\operatorname{com} n$ parâmetros, $p=n$ ) e o modelo sob investigação (com parâmetros, $p<n$ ) avaliados na estimativa de máxima verossimilhança $\hat{\boldsymbol{\beta}}$. Um pequeno valor para a função desvio indica que com um número menor de parâmetros obtém-se um ajuste tão bom quanto o ajuste com o modelo saturado. Denotando por $\hat{\theta}_{i}=\theta_{i}\left(\hat{\mu}_{i}\right)$ e $\hat{\theta}_{i}^{0}=\theta_{i}\left(\hat{\mu}_{i}^{0}\right)$ as estimativas de máxima 
verossimilhança de $\theta$ para os modelos com $p$ parâmetros $(p<n)$ e o modelo saturado $(p=n)$, respectivamente, a função desvio fica dada por

$$
D(\boldsymbol{y} ; \hat{\boldsymbol{\mu}})=2 \sum_{i=1}^{n}\left\{y_{i}\left(\hat{\theta}_{i}^{0}-\hat{\theta}_{i}\right)+\left[b\left(\hat{\theta}_{i}\right)-b\left(\hat{\theta}_{i}^{0}\right)\right]\right\} .
$$

A Tabela 3.2.2 apresenta a função desvio para os modelos mais conhecidos.

Tabela 3.2.2: Função desvio para os modelos mais conhecidos.

\begin{tabular}{|c|c|c|}
\hline Modelo & Função desvio & \\
\hline Normal & $D(\boldsymbol{y} ; \hat{\boldsymbol{\mu}})=\sum_{i=1}^{n}\left(y_{i}-\hat{\mu}_{i}\right)^{2}$ & \\
\hline \multirow[t]{2}{*}{ Poisson } & $D(\boldsymbol{y} ; \hat{\boldsymbol{\mu}})=2 \sum_{i=1}^{n}\left\{y_{i} \ln \left(\frac{y_{i}}{\hat{\mu}_{i}}\right)-\frac{y_{i}}{\hat{\mu}_{i}}\right\}$ & \\
\hline & $-n_{i} \ln \left(\hat{\mu}_{i}\right)$ & se $y_{i}=n_{i}$ \\
\hline Binomial & $D\left(y_{i} ; \hat{\mu}_{i}\right)^{1}=\left\{\begin{array}{l}-n_{i} \ln \left(1-\hat{\mu}_{i}\right) \\
y_{i} \ln \left(\frac{y_{i}}{n_{i} \hat{\mu}_{i}}\right)+\left(n_{i}-y_{i}\right) \ln \left(\frac{1-\frac{y_{i}}{n_{i}}}{1-\hat{\mu}_{i}}\right)\end{array}\right.$ & $\begin{array}{l}\text { se } y_{i}=0 \\
\text { se } 0<y_{i}<n_{i}\end{array}$ \\
\hline Gama & $D(\boldsymbol{y} ; \hat{\boldsymbol{\mu}})=2 \sum_{i=1}^{n}\left\{-\ln \left(\frac{y_{i}}{\hat{\mu}_{i}}\right)+\frac{y_{i}-\hat{\mu}_{i}}{\hat{\mu}_{i}}\right\}$ & \\
\hline Normal Inversa & $D(\boldsymbol{y} ; \hat{\boldsymbol{\mu}})=\sum_{i=1}^{n}\left\{\frac{\left(y_{i} \hat{\boldsymbol{\mu}}_{i}\right)^{2}}{y_{i} \hat{\mu}_{i}^{2}}\right\}$ & \\
\hline
\end{tabular}

Usualmente, compara-se o valor da função desvio com os percentis da distribuição qui-quadrado com $n-p$ graus de liberdade mas, em geral, $D(\boldsymbol{y} ; \hat{\boldsymbol{\mu}})$ não segue assintoticamente uma distribuição $\chi_{n-p}^{2}$. Para o caso binomial quando $n$ é fixo e $n_{i} \rightarrow \infty$ para cada $i$, sob a hipótese de que o modelo é verdadeiro, $D(\boldsymbol{y} ; \hat{\boldsymbol{\mu}}) \sim \chi_{n-p}^{2}$. Isso não vale quando $n \rightarrow \infty$ e $n_{i} \mu_{i}\left(1-\mu_{i}\right)$ permanece limitado. No caso do modelo de Poisson, 
quando $\mu_{i} \rightarrow \infty$ para todo $i$, tem-se que $D(\boldsymbol{y} ; \hat{\boldsymbol{\mu}}) \sim \chi_{n-p}^{2}$. No caso normal, com ligação identidade e $\sigma^{2}$ fixo, é conhecido que $D(\boldsymbol{y} ; \hat{\mu}) \sim \sigma^{2} \chi_{n-p}^{2}$. Como $\mathrm{E}\left(\chi_{k}^{2}\right)=k$, segue-se que um valor da função desvio próximo de $n-p$ pode ser uma indicação de que o modelo está bem ajustado. Em geral, para os casos em que $D^{*}(\boldsymbol{y} ; \hat{\boldsymbol{\mu}})$ depende do parâmetro de dispersão $\phi^{-1}$, tem-se que $D^{*}(\boldsymbol{y} ; \hat{\boldsymbol{\mu}}) \sim \chi_{n-p}^{2}$ quando $\phi \rightarrow \infty$, veja Jørgensen (1987). Ou seja, quando a dispersão é pequena, é razoável comparar o valor observado de $D(\boldsymbol{y} ; \hat{\boldsymbol{\mu}})$ com os percentis da distribuição $\chi_{n-\boldsymbol{p}}^{2}$. Em particular, para o caso normal linear, $\sum_{i=1}^{n}\left(y_{i}-\hat{\mu}_{i}\right)^{2} \sim \sigma^{2} \chi_{n-p}^{2}$ quando $\sigma^{2} \rightarrow 0$. Para o modelo gama, a função desvio é bem aproximada por uma distribuição $\chi_{n-p}^{2}$ quando o coeficiente de variação estiver próximo de zero.

\subsubsection{Estimação dos parâmetros}

A função escore e a matriz de informação de Fisher para o parâmetro $\boldsymbol{\beta}$ no modelo linear generalizado são dadas, respectivamente, por

$$
\begin{gathered}
\mathrm{U}(\boldsymbol{\beta})=\frac{\partial}{\partial \boldsymbol{\beta}} L(\boldsymbol{\beta} ; \boldsymbol{y})=\phi \boldsymbol{X}^{T} \boldsymbol{W}^{1 / 2} \boldsymbol{V}^{-1 / 2}(\boldsymbol{y}-\boldsymbol{\mu}) \mathrm{e} \\
\mathbf{K}(\boldsymbol{\beta})=\mathrm{E}\left\{-\frac{\partial^{2}}{\partial \boldsymbol{\beta} \partial \boldsymbol{\beta}^{T}} L(\boldsymbol{\beta} ; \boldsymbol{y})\right\}=\phi \boldsymbol{X}^{T} \boldsymbol{W} \boldsymbol{X},
\end{gathered}
$$

onde $\boldsymbol{X}$ é uma matriz $(n \times p)$ de posto completo cujas linhas serão denotadas por $\boldsymbol{x}_{i}^{T}, i=$ $1,2, \ldots, n, W=\operatorname{diag}\left\{w_{1}, w_{2}, \ldots, w_{n}\right\}$ é uma matriz de pesos com $w_{i}=\left(d \mu_{i} / d \eta_{i}\right)^{2} / V_{i}$, $\boldsymbol{V}=\operatorname{diag}\left\{V_{1}, V_{2}, \ldots, V_{n}\right\}, \boldsymbol{y}=\left(y_{1}, y_{2}, \ldots, y_{n}\right)^{T}$ e $\boldsymbol{\mu}=\left(\mu_{1}, \mu_{2}, \ldots, \mu_{n}\right)^{T}$. Em particular, para ligações canônicas, as expressões (3.2.3) e (3.2.4) tornam-se mais simples e ficam dadas por

$$
\begin{aligned}
\mathrm{U}(\boldsymbol{\beta}) & =\phi \boldsymbol{X}^{T}(\boldsymbol{y}-\boldsymbol{\mu}) \mathrm{e} \\
\mathbf{K}(\boldsymbol{\beta}) & =\phi \boldsymbol{X}^{T} \boldsymbol{V} \boldsymbol{X} .
\end{aligned}
$$


Para determinar a estimativa de máxima verossimilhança de $\boldsymbol{\beta}$, utiliza-se o processo iterativo de Newton-Raphson expandindo-se a função de escore $\mathrm{U}(\boldsymbol{\beta})$ em série de Taylor até a primeira ordem, em torno de um valor inicial $\beta^{(0)}$, isto é,

$$
\mathrm{U}(\boldsymbol{\beta}) \cong \mathrm{U}\left(\boldsymbol{\beta}^{(0)}\right)+\mathrm{U}^{\prime}\left(\boldsymbol{\beta}^{(0)}\right)\left(\boldsymbol{\beta}-\boldsymbol{\beta}^{(0)}\right)
$$

onde $\mathrm{U}^{\prime}(\boldsymbol{\beta})$ denota a primeira derivada de $\mathrm{U}(\boldsymbol{\beta})$ com respeito a $\boldsymbol{\beta}$. Repetindo-se esse procedimento chega-se ao processo iterativo

$$
\boldsymbol{\beta}^{(m+1)}=\boldsymbol{\beta}^{(m)}+\left\{-\mathbf{U}^{\prime}\left(\boldsymbol{\beta}^{(m)}\right)\right\}^{-1} \mathbf{U}\left(\boldsymbol{\beta}^{(m)}\right)
$$

onde $m=0.1, \ldots$ Como a matriz $-\mathbf{U}^{\prime}(\boldsymbol{\beta})$ pode não ser positiva definida, é mais coveniente aplicar o método de scoring de Fisher substituindo-se a matriz - $\mathbf{U}^{\prime}(\boldsymbol{\beta})$ pelo seu valor esperado $\mathbf{K}(\boldsymbol{\beta})$, isto leva ao processo iterativo

$$
\boldsymbol{\beta}^{(m+1)}=\boldsymbol{\beta}^{(m)}+\mathbf{K}^{-1}\left(\boldsymbol{\beta}^{(m)}\right) \mathbf{U}\left(\boldsymbol{\beta}^{(m)}\right)
$$

onde $m=0,1, \ldots$. Esse processo iterativo pode ser escrito como um processo iterativo de mínimos quadrados reponderados na seguinte forma:

$$
\boldsymbol{\beta}^{(m+1)}=\left(\boldsymbol{X}^{T} \boldsymbol{W}^{(m)} \boldsymbol{X}\right)^{-1} \boldsymbol{X}^{T} \boldsymbol{W}^{(m)} \boldsymbol{z}^{(m)},
$$

onde $m=0,1, \ldots$ e $\boldsymbol{z}=\boldsymbol{\eta}+\boldsymbol{W}^{-1 / 2} \boldsymbol{V}^{-1 / 2}(\boldsymbol{y}-\boldsymbol{\mu})$. Note que $\boldsymbol{z}$ desempenha o papel de uma variável dependente modificada enquanto $W$ é uma matriz de pesos que muda a cada passo do processo iterativo. A convergência de (3.2.9) ocorre em um número finito de passos independente do valor inicial (veja, por exemplo, Wedderburn, 1976). Usualmente o processo iterativo (3.2.9) é inicializado com $\boldsymbol{\eta}^{(0)}=g^{-1}(\boldsymbol{y})$. Sob condições gerais de regularidade (veja, por exemplo, Sen e Singer, 1993, Capítulo 7) mostra-se que $\hat{\boldsymbol{\beta}}$ é um estimador consistente, eficiente e que

$$
\sqrt{n}(\hat{\boldsymbol{\beta}}-\boldsymbol{\beta}) \stackrel{\mathcal{D}}{\longrightarrow} \mathrm{N}\left\{0, \phi^{-1} \boldsymbol{\Sigma}^{-1}(\boldsymbol{\beta})\right\}
$$


onde $\boldsymbol{\Sigma}(\boldsymbol{\beta})=\lim _{n \rightarrow \infty} \frac{1}{n}\left(\boldsymbol{X}^{T} \boldsymbol{W} \boldsymbol{X}\right)$, sendo $\boldsymbol{\Sigma}(\boldsymbol{\beta})$ uma matriz positiva definida. Uma outra referência importante sobre as propriedades assintóticas dos estimadores de máxima verossimilhança dos parâmetros de um modelo linear generalizado é Fahrmeir e Kaufmann (1985).

Por outro lado, fazendo-se

$$
\frac{\partial}{\partial \phi} L(\boldsymbol{\beta}, \phi ; \boldsymbol{y})=0
$$

chega-se à seguinte solução:

$$
\sum_{i=1}^{n} c^{\prime}\left(y_{i}, \hat{\phi}\right)=\frac{1}{2} D(\boldsymbol{y} ; \hat{\boldsymbol{\mu}})-\sum_{i=1}^{n}\left\{y_{i} \hat{\theta}_{i}^{0}-b\left(\hat{\theta}_{i}^{0}\right)\right\},
$$

onde $c^{\prime}\left(y_{i}, \phi\right)$ é a primeira derivada de $c\left(y_{i}, \phi\right)$ em relação a $\phi$ e $D(\boldsymbol{y} ; \hat{\boldsymbol{\mu}})$ é a função desvio do modelo sob investigação. Pode-se verificar facilmente que a estimativa de máxima verossimilhança de $\phi$ para os modelos normal e normal inversa são dadas por

$$
\hat{\phi}=\frac{n}{D(\boldsymbol{y} ; \hat{\boldsymbol{\mu}})} .
$$

Para o modelo gama, $\hat{\phi}$ sai da equação

$$
2 n\{\ln (\hat{\phi})-\psi(\hat{\phi})\}=D(\boldsymbol{y} ; \hat{\boldsymbol{\mu}})
$$

onde $\psi(\phi)=\Gamma^{\prime}(\phi) / \Gamma(\phi)$ é a função digama. Usando-se a aproximação dada em Cordeiro e McCullagh $(1991), \psi(\phi) \cong \ln (\phi)-(1 / 2 \phi)-\left(1 / 12 \phi^{2}\right)$, chega-se à solução

$$
\hat{\phi} \cong \frac{n+\left\{n^{2}+\frac{2 n}{3} D(\boldsymbol{y} ; \hat{\boldsymbol{\mu}})\right\}^{1 / 2}}{2 D(\boldsymbol{y} ; \hat{\boldsymbol{\mu}})}
$$

Essa estimativa não é consistente quando a suposição de distribuição gama não é verdadeira, nesse caso, um estimador consistente baseado na estatística de Pearson é dado por

$$
\tilde{\phi}^{-1}=\frac{1}{n-p} \sum_{i=1}^{n}\left(\frac{y_{i}-\hat{\mu}_{i}}{\hat{\mu}_{i}}\right)^{2}
$$


supondo que $\boldsymbol{\beta}$ tem sido estimado consistentemente. É fácil verificar que

$$
\operatorname{Var}(\hat{\phi})=\left\{-\sum_{i=1}^{n} c^{\prime \prime}\left(y_{i}, \phi\right)\right\}^{-1},
$$

onde $c^{\prime \prime}\left(y_{i}, \phi\right)$ é a segunda derivada de $c\left(y_{i}, \phi\right)$ em relação a $\phi$. Discussões sobre a estimação do parâmetro de dispersão $\phi^{-1}$, quando o mesmo é desconhecido, podem ser encontradas em Cordeiro e McCullagh (1991).

\subsubsection{Estimação com parâmetros restritos}

Em muitas situações práticas pode haver necessidade de se estimar os parâmetros de um modelo linear generalizado sob restrições de igualdade e/ou desigualdade lineares. Para determinar as estimativas de máxima verossimilhança de $\beta$ sujeito à restrição $\boldsymbol{R} \boldsymbol{\beta}=\boldsymbol{r}$, onde $\boldsymbol{R}$ e $\boldsymbol{r}$ são definidos como na Seção 2.3, é necessário usar algum processo iterativo, que pode ser mais complexo. Nyquist (1991) propõe um processo iterativo para estimar $\boldsymbol{\beta}$ sujeito à restrição $\boldsymbol{R} \boldsymbol{\beta}=\boldsymbol{r}$ usando a função penalizada

$$
F(\boldsymbol{\beta}, \lambda)=\sum_{i=1}^{n} \phi\left\{y_{i} \theta_{i}-b\left(\theta_{i}\right)\right\}+\sum_{i=1}^{n} c\left(y_{i}, \phi\right)-\frac{1}{2} \sum_{j=1}^{k} \lambda_{j}\left(r_{j}-\boldsymbol{R}_{j}^{T} \boldsymbol{\beta}\right)^{2},
$$

onde $\lambda=\left(\lambda_{1}, \lambda_{2}, \ldots, \lambda_{k}\right)^{T}$ é o vetor de multiplicadores de Lagrange, $r_{j}$ é a $j$-ésima componente do vetor $r, \boldsymbol{R}_{j}^{T}$ é a $j$-ésima linha da matriz $\boldsymbol{R}$ e $\phi$ é um parâmetro de perturbação tratado como conhecido. Determina-se o valor de $\boldsymbol{\beta}$ que maximiza $F(\boldsymbol{\beta}, \lambda)$ para valores $\lambda_{j}, j=1,2, \ldots, k$, fixados e positivos. Esse procedimento leva ao processo iterativo

$$
\begin{aligned}
\overline{\boldsymbol{\beta}}^{(m+1)} & =\hat{\boldsymbol{\beta}}^{(m+1)}+\left(\boldsymbol{X}^{T} \boldsymbol{W}^{(m)} \boldsymbol{X}\right)^{-1} \boldsymbol{R}^{T} \times \\
& \times\left\{\boldsymbol{R}\left(\boldsymbol{X}^{T} \boldsymbol{W}^{(m)} \boldsymbol{X}\right)^{-1} \boldsymbol{R}^{T}\right\}^{-1}\left\{\boldsymbol{r}-\boldsymbol{R} \hat{\boldsymbol{\beta}}^{(m+1)}\right\},
\end{aligned}
$$

$m=0,1, \ldots$, onde $\hat{\boldsymbol{\beta}}^{(m+1)}$ é (3.2.9) avaliado na estimativa restrita $\overline{\boldsymbol{\beta}}^{(m)}$. A matriz de covariância assintótica de $\overline{\boldsymbol{\beta}}$ é dada por

$$
\operatorname{Var}(\overline{\boldsymbol{\beta}})=\left(\boldsymbol{X}^{T} \boldsymbol{W} \boldsymbol{X}\right)^{-1}\left\{\mathrm{I}_{p}-\boldsymbol{R}^{T}\left\{\boldsymbol{R}\left(\boldsymbol{X}^{T} \boldsymbol{W} \boldsymbol{X}\right)^{-1} \boldsymbol{R}^{T}\right\}^{-1} \boldsymbol{R}\left(\boldsymbol{X}^{T} \boldsymbol{W} \boldsymbol{X}\right)^{-1}\right\}
$$


onde $\mathbf{I}_{p}$ é a matriz identidade de ordem $p$.

Por outro lado, para estimar $\boldsymbol{\beta}$ sujeito à restrição $\boldsymbol{R} \boldsymbol{\beta} \geq \boldsymbol{r}$, no caso dos modelos lineares generalizados com função log verossimilhança côncava, pode-se fazer uma variante do algoritmo descrito na Seção 2.3. Vale ressaltar que os modelos lineares generalizados com ligação canônica garantem a concavidade da log verossimilhança. Inicialmente verifica-se a ocorrência de alguma violação do tipo $\boldsymbol{R}_{i}^{T} \boldsymbol{\beta}<r_{i}, i=1,2, \ldots, k$. Suponha, por exemplo, que ocorreu apenas uma violação, digamos $\boldsymbol{R}_{1}^{T} \boldsymbol{\beta}<r_{1}$, então a estimativa restrita de máxima verossimilhança $\tilde{\boldsymbol{\beta}}$, sai do ajuste do modelo linear generalizado sujeito à restrição $\boldsymbol{R}_{1}^{T} \boldsymbol{\beta}=r_{1}$, onde $\boldsymbol{R}_{1}^{T}$ e $r_{1}$ representam, respectivamente, a primeira linha da matriz $\boldsymbol{R}$ e a primeira componente do vetor $\boldsymbol{r}$. Nesse caso, pode-se usar qualquer um dos processos iterativos dados em (3.2.9) e (3.2.11). No caso de duas ou mais violações, deve-se ajustar o modelo linear generalizado sob todas as combinações de restrições de igualdades lineares. A estimativa restrita $\tilde{\boldsymbol{\beta}}$, sai do ajuste que apresentar o menor valor para a função desvio $D(\boldsymbol{y} ; \hat{\boldsymbol{\mu}})$, dentre aqueles que não apresentaram violação. Portanto, se ocorrem $m$ violações, tem-se $2^{m}-1$ problemas de minimização de um modelo linear generalizado sujeito à restrições de igualdades lineares.

Outra maneira de determinar o estimador restrito de máxima verossimilhança de $\boldsymbol{\beta}$ sujeito à restrição $\boldsymbol{R} \boldsymbol{\beta} \geq \boldsymbol{r}$, é usar o algoritmo SQP (Sequential Quadratic Programming) proposto por Fahrmeir e Klinger (1994). Esse procedimento resolve o problema de otimização

$$
\max _{\boldsymbol{\beta}} L(\boldsymbol{\beta} ; \boldsymbol{y}) \quad \text { sujeito a } \quad \boldsymbol{R} \boldsymbol{\beta} \geq \boldsymbol{r}
$$

usando a função Lagrangeana

$$
\begin{aligned}
\mathcal{L}(\boldsymbol{\beta}, \boldsymbol{\lambda}) & =L(\boldsymbol{\beta} ; \boldsymbol{y})-\lambda^{T}(\boldsymbol{R} \boldsymbol{\beta}-\boldsymbol{r}) \\
& =L(\boldsymbol{\beta} ; \boldsymbol{y})-\sum_{i=1}^{k} \lambda_{i}\left(\boldsymbol{R}_{i}^{T} \boldsymbol{\beta}-r_{i}\right)
\end{aligned}
$$

onde $\boldsymbol{\lambda}=\left(\lambda_{1}, \lambda_{2}, \ldots, \lambda_{k}\right)^{T} \geq 0$ é o vetor de multiplicadores de Lagrange, $\boldsymbol{R}_{i}^{T}$ é a $i$-ésima 
linha da matriz $\boldsymbol{R}$ e $r_{i}$ é a $i$-ésima componente do vetor $r$. As condições para que $\tilde{\boldsymbol{\beta}}$ seja um máximo local são:

1. $\boldsymbol{R}_{i}^{T} \tilde{\boldsymbol{\beta}}=r_{i}$, para $i \in I \subseteq\{1,2, \ldots, k\}, \boldsymbol{R}_{i}^{T} \tilde{\boldsymbol{\beta}}>r_{i}$, para $i \notin I$, isto é, $\tilde{\boldsymbol{\beta}}$ é um ponto admissível;

2. Existe um $\tilde{\lambda}=\left(\tilde{\lambda}_{1}, \tilde{\lambda}_{2}, \ldots, \tilde{\lambda}_{k}\right)^{T} \geq 0 \operatorname{com} \partial \mathcal{L}(\boldsymbol{\beta}, \tilde{\lambda}) /\left.\partial \beta\right|_{\tilde{\boldsymbol{\beta}}}=0$, isto é, $\tilde{\boldsymbol{\beta}}$ é um ponto estacionário;

3. $\boldsymbol{s}^{T}\left\{\partial^{2} L(\boldsymbol{\beta} ; \boldsymbol{y}) /\left.\partial \boldsymbol{\beta} \partial \boldsymbol{\beta}^{T}\right|_{\tilde{\boldsymbol{\beta}}}\right\} s<0$, para todo $s \neq 0$ e $\boldsymbol{s} \in\left\{s: \boldsymbol{R}_{i}^{T} s=0, i \in I, \tilde{\lambda}_{i} \geq\right.$ 0 e $\left.\boldsymbol{R}_{i}^{T} s>0, i \in I . \tilde{\lambda}_{i}=0\right\}$.

O algoritmo SQP é um processo iterativo que gera uma sequência de estimadores $\boldsymbol{\beta}^{(m)}, m=0,1, \ldots$, os quais são soluções de problemas de programação quadrática, que converge para $\tilde{\boldsymbol{\beta}}$. Dado o valor $\boldsymbol{\beta}^{(m)}$ no passo $m, \boldsymbol{\beta}^{(m+1)}$ é o valor de $\boldsymbol{\beta}$ que maximiza

$$
\begin{aligned}
Q\left(\boldsymbol{\beta} ; \boldsymbol{\beta}^{(m)}\right) & =L\left(\boldsymbol{\beta}^{(m)} ; \boldsymbol{y}\right)+\mathbf{U}\left(\boldsymbol{\beta}^{(m)}\right)^{T}\left(\boldsymbol{\beta}-\boldsymbol{\beta}^{(m)}\right)- \\
& -\frac{1}{2}\left(\boldsymbol{\beta}-\boldsymbol{\beta}^{(m)}\right)^{T} \mathbf{K}\left(\boldsymbol{\beta}^{(m)}\right)\left(\boldsymbol{\beta}-\boldsymbol{\beta}^{(m)}\right) \text { sujeito a } \boldsymbol{R} \boldsymbol{\beta} \geq \boldsymbol{r},
\end{aligned}
$$

onde $\mathbf{U}\left(\boldsymbol{\beta}^{(m)}\right)$ e $\mathbf{K}\left(\boldsymbol{\beta}^{(m)}\right)$ são a função de escore e a matriz de informação de Fisher, respectivamente, avaliadas em $\boldsymbol{\beta}^{(m)}$. Como foi visto no Capítulo 2, existem vários métodos para resolver (3.2.13) (veja, por exemplo, Bazaraa et al., 1993). O algoritmo SQP para resolver o problema de otimização (3.2.12) resume-se nos seguintes passos:

1. Faça $m=0$ e $\boldsymbol{\beta}^{(0)}=\hat{\boldsymbol{\beta}}$. Se $\boldsymbol{R} \hat{\boldsymbol{\beta}} \geq \boldsymbol{r}$ parar e tomar $\tilde{\boldsymbol{\beta}}=\hat{\boldsymbol{\beta}}$. Caso contrário:

2. Calcule $\boldsymbol{\beta}^{(m+1)}$ resolvendo o PPQ (3.2.13) e;

3. Se $\left\|\boldsymbol{\beta}^{(m+1)}-\boldsymbol{\beta}^{(m)}\right\| \leq \epsilon$ parar e tomar $\tilde{\boldsymbol{\beta}}=\boldsymbol{\beta}^{(m+1)}$. Caso contrário, faça $m=m+1$ e volte ao passo 2 . 
A convergência da sequência $\boldsymbol{\beta}^{(m)}$, produzida pelo algoritmo SQP, para o estimador de máxima verossimilhança $\tilde{\boldsymbol{\beta}}$, pode ser garantida sob as seguintes condições (veja Fahrmeir e Klinger, 1994): a função objetiva é duas vezes contínua e diferenciável; o estimador de máxima verossimilhança não restrito $\hat{\boldsymbol{\beta}}$, existe e é único e; a log verossimilhança é côncava para algum subconjunto $D \subset \mathbb{R}^{p}$ contendo $\tilde{\boldsymbol{\beta}}$ e $D \cap\{\boldsymbol{\beta}: \boldsymbol{R} \boldsymbol{\beta} \geq \boldsymbol{r}\} \neq \emptyset$. Essas condições são satisfeitas para uma grande variedade de modelos lineares generalizados, em particular, todos os modelos lineares generalizados com ligação canônica.

Note que quando $\boldsymbol{\beta}^{(0)}=\hat{\boldsymbol{\beta}}$ no passo 1 do algoritmo $\mathrm{SQP}$, então $\mathrm{U}\left(\boldsymbol{\beta}^{(0)}\right)=0$, de modo que resolver (3.2.13) no passo 2, é equivalente a

$$
\min _{\boldsymbol{\beta}}\left\{(\hat{\boldsymbol{\beta}}-\boldsymbol{\beta})^{T} \mathrm{~K}(\hat{\boldsymbol{\beta}})(\hat{\boldsymbol{\beta}}-\boldsymbol{\beta})\right\} \text { sujeito a } \boldsymbol{R} \boldsymbol{\beta} \geq \boldsymbol{r} .
$$

Para o caso de restrições não negativas, Piegorsch (1990) propõe o cálculo do estimador de máxima verossimilhança restrito $\tilde{\boldsymbol{\beta}}$ dessa maneira, o que é equivalente a parar o algoritmo SQP na primeira iteração, portanto, mais iterações podem melhorar substancialmente o estimador de máxima verossimilhança restrito. Outro método para determinar o estimador de máxima verossimilhança restrito $\tilde{\boldsymbol{\beta}}$ é apresentado em McDonald e Diamond (1990).

\subsection{Hipóteses em desigualdades lineares}

Vamos considerar agora um modelo linear generalizado com o parâmetro $\phi$ conhecido e suponha que queremos testar as hipóteses

$$
H_{0}: \boldsymbol{R} \boldsymbol{\beta}=\boldsymbol{r} \text { versus } H_{1}: \boldsymbol{R} \boldsymbol{\beta} \geq \boldsymbol{r}
$$

com pelo menos uma desigualdade estrita em $H_{1}$, onde $\boldsymbol{R}$ e $\boldsymbol{r}$ são definidos como na Seção 2.3. As estatísticas usuais para testar as hipóteses (3.3.1) são a razão de verossi- 
milhança $\xi_{r v}$, a estatística de Wald $\xi_{w}$ e a estatística escore $\xi_{s r}$. A estatística escore é equivalente à estatística de Kuhn-Tucker definida no Capítulo 2. O objetivo desta seção é apresentar a distribuição nula assintótica dessas três estatísticas. A teoria desenvolvida no Capítulo 2 pode ser estendida para os modelos lineares generalizados. Isso pode ser mostrado de maneira intuitiva como segue. Como foi visto na Seção 3.2.5, para os modelos lineares generalizados sabe-se que

$$
\sqrt{n}(\hat{\boldsymbol{\beta}}-\boldsymbol{\beta}) \stackrel{\mathcal{D}}{\longrightarrow} \mathrm{N}_{p}\left\{0, \phi^{-1} \Sigma^{-1}(\boldsymbol{\beta})\right\}
$$

onde $\Sigma^{-1}(\boldsymbol{\beta})$ é uma matriz positiva definida . Segue-se então que

$$
\sqrt{n}(\boldsymbol{R} \hat{\boldsymbol{\beta}}-\boldsymbol{R} \boldsymbol{\beta}) \stackrel{\mathcal{D}}{\longrightarrow} \mathrm{N}_{k}\left\{0, \phi^{-1} \Omega(\boldsymbol{\beta})\right\}
$$

onde $\Omega(\boldsymbol{\beta})=R \boldsymbol{\Sigma}^{-1}(\boldsymbol{\beta}) \boldsymbol{R}^{T}$. Portanto, para $n$ grande, tem-se aproximadamente o seguinte modelo:

$$
\hat{\boldsymbol{\mu}}=\boldsymbol{\mu}+\boldsymbol{v}, \quad \boldsymbol{v} \sim \mathrm{N}_{k}(0, \Omega),
$$

onde $\boldsymbol{\mu}=\sqrt{n}(\boldsymbol{R} \boldsymbol{\beta}-\boldsymbol{r})$ e assumimos que $\Omega=\Omega(\boldsymbol{\beta})$ está sendo avaliada no verdadeiro valor do parâmetro $\boldsymbol{\beta}_{0}$. Vamos supor que o interesse sob o modelo (3.3.2) seja testar

$$
H_{0}: \boldsymbol{\mu}=\mathbf{0} \text { versus } H_{1}: \boldsymbol{\mu} \geq \mathbf{0}
$$

com pelo menos uma desigualdade estrita em $H_{1}$. A estatística de Wald para testar as hipóteses (3.3.3) fica (vide Seção 2.4) dada por

$$
\begin{aligned}
\xi_{w}^{*} & =\tilde{\boldsymbol{\mu}}^{T} \phi \boldsymbol{\Omega}^{-1} \tilde{\boldsymbol{\mu}} \\
& =\phi \tilde{\boldsymbol{\mu}}^{T}\left\{\boldsymbol{R} \boldsymbol{\Sigma}^{-1}(\boldsymbol{\beta}) \boldsymbol{R}^{T}\right\}^{-1} \tilde{\boldsymbol{\mu}} \\
& =n \phi(\boldsymbol{R} \tilde{\boldsymbol{\beta}}-\boldsymbol{r})^{T}\left\{\boldsymbol{R} \boldsymbol{\Sigma}^{-1}(\boldsymbol{\beta}) \boldsymbol{R}^{T}\right\}^{-1}(\boldsymbol{R} \tilde{\boldsymbol{\beta}}-\boldsymbol{r}) .
\end{aligned}
$$

Quando $\Sigma(\boldsymbol{\beta})$ é assumida conhecida, segue do Teorema 2.2 que a distribuição nula de $\xi_{w}^{*}$ para todo $c>0$ é aproximadamente para $n$ grande dada por

$$
\begin{aligned}
& P\left(\xi_{w}^{*} \geq c\right)=\sum_{\ell=1}^{k} w(k, \ell ; \Omega) P\left(\chi_{\ell}^{2} \geq c\right) \text { e } \\
& P\left(\xi_{w}^{*}=0\right)=w(k, 0 ; \Omega) .
\end{aligned}
$$


Por outro lado, a estatística de Wald para testar as hipóteses

$$
H_{0}: \boldsymbol{R} \boldsymbol{\beta}=r \text { versus } H_{1}: \boldsymbol{R} \boldsymbol{\beta} \geq r
$$

com pelo menos uma desigualdade estrita em $H_{1}$, no modelo linear generalizado sob investigação, é dada por

$$
\begin{aligned}
\xi_{w} & =(\boldsymbol{R} \tilde{\boldsymbol{\beta}}-\boldsymbol{r})^{T}\{\hat{\operatorname{Var}}(\boldsymbol{R} \hat{\boldsymbol{\beta}})\}^{-1}(\boldsymbol{R} \tilde{\boldsymbol{\beta}}-\boldsymbol{r}) \\
& =\phi(\boldsymbol{R} \tilde{\boldsymbol{\beta}}-\boldsymbol{r})^{T}\left\{\boldsymbol{R}\left(\boldsymbol{X}^{T} \hat{\boldsymbol{W}} \boldsymbol{X}\right)^{-1} \boldsymbol{R}^{T}\right\}^{-1}(\boldsymbol{R} \tilde{\boldsymbol{\beta}}-\boldsymbol{r})
\end{aligned}
$$

Como $\Sigma=\lim _{n \rightarrow \infty} \frac{1}{n}\left(\boldsymbol{X}^{T} \boldsymbol{W} \boldsymbol{X}\right)$ e $\hat{\boldsymbol{W}}$ é um estimador consistente de $\boldsymbol{W}$ segue portanto que $n\left(\boldsymbol{X}^{T} \hat{\boldsymbol{W}} \boldsymbol{X}\right)^{-1}$ é um estimador consistente de $\boldsymbol{\Sigma}^{-1}$. Logo. podemos escrever $\xi_{w}=$ $\xi_{w}^{*}+o_{p}(1)$ de onde, para $c>0$, segue que

$$
\begin{aligned}
\lim _{n \rightarrow \infty} P\left(\xi_{w} \geq c\right) & =\sum_{\ell=1}^{k} w(k, \ell ; \Omega) P\left(\chi_{\ell}^{2} \geq c\right) \mathrm{e} \\
\lim _{n \rightarrow \infty} P\left(\xi_{w}=0\right) & =w(k, 0 ; \Omega) .
\end{aligned}
$$

Os pesos $w(k, \ell ; \Omega)$ 's são os mesmos do Teorema 2.2 , que dependem de $\Omega$. Na prática, entretanto, os pesos dependem agora de $\boldsymbol{\beta}$, mesmo sob $H_{0}$, fazendo com que o lado direito de (3.3.8) assuma um valor para cada $\boldsymbol{\beta}$, para $c$ fixado. Em outras palavras, a distribuição nula assintótica de $\xi_{w}$ não é mais única, como ocorre para o caso em que $\boldsymbol{\Omega}$ não depende de $\boldsymbol{\beta}$. Uma solução possível, é procurar a distribuição nula menos favorável, o que pode ter um elevado custo computacional uma vez que precisamos varrer todos os possíveis valores tais que $\boldsymbol{R} \boldsymbol{\beta}=\boldsymbol{r}$. A solução mais plausível é substituir $\boldsymbol{\beta}$ por uma estimativa consistente, por exemplo $\hat{\boldsymbol{\beta}}$. Fahrmeir e Klinger (1994) verificaram, através de simulações, que as diferenças entre a probabilidade de rejeição da distribuição nula menos favorável e aquela avaliada em $\hat{\boldsymbol{\beta}}$ são desprezíveis. Paula e Sen (1995) e Paula (1999) verificaram, para alguns modelos lineares generalizados, que estruturas particulares para a matriz $\boldsymbol{X}$ eliminam a dependência de $\Omega$ em $\boldsymbol{\beta}$ sob a hipótese nula, o que garante a unicidade da distribuição nula assintótica. Assim, para grandes amostras 
e $c>0$ a distribuição nula de $\xi_{w}$ fica aproximadamente dada por

$$
\begin{aligned}
& P\left(\xi_{w} \geq c\right)=\sum_{\ell=1}^{k} w(k, \ell ; \hat{\Omega}) P\left(\chi_{\ell}^{2} \geq c\right) \mathrm{e} \\
& P\left(\xi_{w}=0\right)=w(k, 0 ; \hat{\Omega})
\end{aligned}
$$

onde $\hat{\Omega}=n \boldsymbol{R}\left(\boldsymbol{X}^{T} \hat{W} \boldsymbol{X}\right)^{-1} \boldsymbol{R}^{T}$. O cálculo dos pesos $w(k, \ell ; \hat{\Omega})$ 's está descrito no Apendice $\mathrm{A}$.

A estatística da razão de verossimilhança para testar as hipótese (3.3.6) fica agora dada por

$$
\xi_{r v}=\phi\{D(\boldsymbol{y} ; \overline{\boldsymbol{\mu}})-D(\boldsymbol{y} ; \tilde{\boldsymbol{\mu}})\}
$$

onde $D(\boldsymbol{y} ; \overline{\boldsymbol{\mu}})$ e $D(\boldsymbol{y} ; \tilde{\boldsymbol{\mu}})$ denotam a função desvio sob as hipóteses $H_{0}$ e $H_{1}$, respectivamente. Já a estatística escore toma a forma

$$
\begin{aligned}
\xi_{s r} & =\{\mathbf{U}(\overline{\boldsymbol{\beta}})-\mathbf{U}(\tilde{\boldsymbol{\beta}})\}^{T}\{\hat{\operatorname{V}} \operatorname{ar}(\hat{\boldsymbol{\beta}})\}\{\mathbf{U}(\overline{\boldsymbol{\beta}})-\mathbf{U}(\tilde{\boldsymbol{\beta}})\} \\
& =\phi^{-1}\{\mathbf{U}(\overline{\boldsymbol{\beta}})-\mathbf{U}(\tilde{\boldsymbol{\beta}})\}^{T}\left(\boldsymbol{X}^{T} \hat{\boldsymbol{W}} \boldsymbol{X}\right)^{-1}\{\mathbf{U}(\overline{\boldsymbol{\beta}})-\mathbf{U}(\tilde{\boldsymbol{\beta}})\}
\end{aligned}
$$

A equivalência assintótica entre as estatísticas $\xi_{r v}, \xi_{s r}$ e $\xi_{w}$ é mostrada, por exemplo, em Gouriéroux e Monfort (1995, Capítulo 21), sob as seguintes condições de regularidade:

(a) $L(\boldsymbol{\beta} ; \boldsymbol{y})$ está bem definida numa vizinhança do verdadeiro valor do parâmetro $\boldsymbol{\beta}_{0}$;

(b) os estimadores $\overline{\boldsymbol{\beta}}, \tilde{\boldsymbol{\beta}}$ e $\hat{\boldsymbol{\beta}}$ são consistentes;

(c) $\frac{1}{\sqrt{n}} \mathbf{U}\left(\boldsymbol{\beta}_{0}\right) \stackrel{\mathcal{D}}{\longrightarrow} \mathrm{N}_{p}\left\{0, \Sigma\left(\boldsymbol{\beta}_{0}\right)\right\}$;

(d) $-\frac{1}{n} \partial^{2} L(\boldsymbol{\beta} ; \boldsymbol{y}) /\left.\partial \boldsymbol{\beta} \partial \boldsymbol{\beta}^{T}\right|_{\boldsymbol{\beta}_{0}} \stackrel{q . c .}{\longrightarrow} \boldsymbol{\Sigma}\left(\boldsymbol{\beta}_{0}\right)$;

(e) $\sqrt{n}\left(\hat{\boldsymbol{\beta}}-\boldsymbol{\beta}_{0}\right) \stackrel{\mathcal{D}}{\longrightarrow} \mathrm{N}_{p}\left\{\mathbf{0}, \boldsymbol{\Sigma}^{-1}\left(\boldsymbol{\beta}_{0}\right)\right\}$;

(f) $\boldsymbol{R} \boldsymbol{\beta}_{0} \geq \boldsymbol{r}$;

(g) $\mathrm{U}(\tilde{\boldsymbol{\beta}})+\boldsymbol{R}^{T} \tilde{\boldsymbol{\lambda}}=0$;

(h) $\boldsymbol{R} \tilde{\boldsymbol{\beta}} \geq \boldsymbol{r}$ e $\quad \tilde{\lambda}_{j} \geq 0 \quad(j=1,2, \ldots, k) \mathrm{e}$

(i) $\tilde{\lambda}_{j}\left\{\boldsymbol{R}_{j}^{T} \tilde{\boldsymbol{\beta}}-r_{j}\right\}=0 \quad(j=1,2, \ldots, k)$. 
A condição (a) é implicitamente assumida nas demonstrações de Gouriéroux e Monfort (1995) a fim de permitir a expansão de $L(\boldsymbol{\beta} ; \boldsymbol{y})$ em torno de $\boldsymbol{\beta}_{0}$. Como a consistência de um estimador independe das restrições impostas na maximização da função log verossimilhança, a consistência de $\hat{\boldsymbol{\beta}}$ garante a consistência de $\overline{\boldsymbol{\beta}}$ e $\tilde{\boldsymbol{\beta}}$ e consequentemente a condição (b) é garantida. As condições (c) - (e) são usuais no caso irrestrito (veja, por exemplo, Sen e Singer, 1993, Capítulo 7). A condição (f) é requerida para o verdadeiro valor do parâmetro e as condições (g) - (i) são conhecidas como condições de KuhnTucker de primeira ordem. A concavidade de $L(\boldsymbol{\beta} ; \boldsymbol{y})$ em $\boldsymbol{\beta}$ garante essas três condições. Como vimos na Seção 3.2 os modelos lineares generalizados com ligação canônica têm $L(\boldsymbol{\beta} ; \boldsymbol{y})$ côncava em $\boldsymbol{\beta}$. Wedderburn (1976) discute a concavidade da função log verossimilhança em modelos lineares generalizados com ligação não canônica e mostra que em geral $L(\boldsymbol{\beta} ; \boldsymbol{y})$ é côncava em $\boldsymbol{\beta}$. Assim, podemos dizer, que as condições de regularidade (a) - (i) seguem de uma forma geral na classe dos modelos lineares generalizados o que garante que as três estatísticas $\xi_{w}, \xi_{r v}$ e $\xi_{s r}$ têm a mesma distribuição nula assintótica dada por (3.3.9). Gouriéroux e Monford mostram também que quando o verdadeiro valor do parâmetro satisfaz a condição $\boldsymbol{R} \boldsymbol{\beta}>\boldsymbol{r}$, a distribuição assintótica de $\tilde{\boldsymbol{\beta}}$ é a mesma de $\hat{\boldsymbol{\beta}}$. No entanto, quando o verdadeiro valor do parâmetro está na fronteira, $\boldsymbol{R} \boldsymbol{\beta}=\boldsymbol{r}$, a determinação da distribuição de $\tilde{\boldsymbol{\beta}}$ é bem mais complexa. Em Feder (1968) há uma discussão sobre as propriedades da estatística da razão de verossimilhança quando o verdadeiro valor do parâmetro está próximo da fronteira.

\subsection{Lema de Wolak}

A outra situação de interesse é testar as hipóteses

$$
H_{1}: \boldsymbol{R} \boldsymbol{\beta} \geq \boldsymbol{r} \text { versus } H_{2}-H_{1},
$$


onde $H_{2}: \boldsymbol{\beta} \in \mathbb{R}^{p}$. Nesse caso, pelo fato da matriz $\boldsymbol{\Omega}(\boldsymbol{\beta})=\boldsymbol{R} \boldsymbol{\Sigma}^{-1}(\boldsymbol{\beta}) \boldsymbol{R}^{T}$ depender de $\boldsymbol{\beta}$, mesmo sob $H_{0}$, a distribuição nula menos favorável pode não ser alcançada quando todas as desigualdades em $H_{0}$ são safisfeitas na forma de igualdades, isto é, sob $\boldsymbol{R} \boldsymbol{\beta}=\boldsymbol{r}$. Esse problema foi estudado, em particular, por Wolak (1991) que propôs um lema bastante geral que será apresentado a seguir. Para a validade dos resultados, a principal condição de regularidade exigida é que $\sqrt{n}(\hat{\boldsymbol{\beta}}-\boldsymbol{\beta}) \stackrel{\mathcal{D}}{\longrightarrow} \mathrm{N}_{p}\left\{0, \phi^{-1} \boldsymbol{\Sigma}^{-1}(\boldsymbol{\beta})\right\}$ e que $\boldsymbol{\Sigma}(\boldsymbol{\beta})$ seja positiva definida, o que é verificado para os modelos lineares generalizados.

A estatística da razão de verossimilhança para testar as hipótese (3.4.1) fica agora dada por

$$
\xi_{r v}=\phi\{D(\boldsymbol{y} ; \tilde{\boldsymbol{\mu}})-D(\boldsymbol{y} ; \hat{\boldsymbol{\mu}})\}
$$

Antes de enunciar o lema de Wolak, é necessário as seguintes definições: sejam $C=$ $\left\{\boldsymbol{\beta} \in \mathbb{R}^{p}: \boldsymbol{R} \boldsymbol{\beta} \geq \boldsymbol{r}\right\}, C^{i}=\left\{\boldsymbol{\beta} \in \mathbb{R}^{p}: \boldsymbol{R} \boldsymbol{\beta}>\boldsymbol{r}\right\}$ e $C^{b}=C-C^{i}$. O conjunto $C^{b}$ é particionado nos subconjuntos $A=\left\{\boldsymbol{\beta} \in \mathbb{R}^{p}: \boldsymbol{\beta} \in C^{b}\right.$ e $\boldsymbol{R}_{i}^{T} \boldsymbol{\beta}=r_{i}$ para somente um $i \in I\}$, onde $I=\{1,2, \ldots, k\}$ e $B=C^{b}-A$. Para todo $\boldsymbol{\beta} \in C^{b}$, seja $\boldsymbol{R}_{b}$ uma submatriz de $\boldsymbol{R}$ consistindo de $m \leq k$ linhas de $\boldsymbol{R}$.

Lema 3.1 (Lema de Wolak). Para o problema de testar as hipóteses (3.4.1) temos que

1. Para todo $\boldsymbol{\beta} \in C^{i}, \lim _{n \rightarrow \infty} P\left(\xi_{r v}=0 \mid \boldsymbol{\beta}\right)=1$;

2. Para todo $c>0$ e $\boldsymbol{\beta} \in C^{b}$ tal que $\boldsymbol{R}_{b}$ é de posto completo,

$$
\lim _{n \rightarrow \infty} P\left(\xi_{r v} \geq c \mid \boldsymbol{\beta}\right)=\sum_{\ell=1}^{m} w\left(m, m-\ell ; \Omega_{b}\right) P\left(\chi_{\ell}^{2} \geq c\right)
$$

onde $\boldsymbol{\Omega}_{b}=\boldsymbol{R}_{b} \Sigma^{-1}(\boldsymbol{\beta}) \boldsymbol{R}_{b}^{T}$; 
3. Para todo $\boldsymbol{\beta} \in B, \boldsymbol{\beta}^{*} \in A$ e c $>0$ fixo,

$$
\lim _{n \rightarrow \infty} P\left(\xi_{r v} \geq c \mid \boldsymbol{\beta}\right) \geq \lim _{n \rightarrow \infty} P\left(\xi_{r v} \geq c \mid \boldsymbol{\beta}^{*}\right)
$$

A parte 1 desse lema afirma que todo $\boldsymbol{\beta} \in C^{i}$, o interior do conjunto de restrições, pode ser retirado do processo de busca do valor menos favorável, uma vez que $\xi_{r v}$ converge para zero em probabilidade para esses valores de $\boldsymbol{\beta}$. A parte 3 elimina certos elementos $\boldsymbol{\beta} \in C^{b}$ desse processo de busca, enquanto a parte 2 caracteriza a distribuição nula assintótica de $\xi_{r v}$ para todo $\boldsymbol{\beta} \in C^{b}$. Esse lema requer que o valor menos favorável seja um elemento do conjunto $B$. Portanto, uma vez determinada a matriz $\boldsymbol{R}_{b}$, deve-se procurar qual conjunto de valores para $\boldsymbol{\beta} \in C^{b}$ fornece o maior valor para (3.4.3). A determinação de $m$, porém, não é trivial. Sabe-se apenas que $2 \leq m \leq k$, o que exige o desenvolvimento de um algoritmo de busca da situação menos favorável para cada $m \geq 2$ e daí a obtenção do valor de $m$ que maximiza (3.4.3). Fahrmeir e Klinger (1994) verificaram, através de simulações, que a situação menos favorável é em geral alcançada quando $m=k$. Todavia, isso não elimina a necessidade de uma busca para valores de $m<k$. Wolak (1991) mostra que essa distribuição é sempre alcançada para $m=k$, se o parâmetro verdadeiro pertencer à fronteira do espaço paramétrico sob $H_{0}$. Um outro resultado relevante, verificado por Fahrmeir e Klinger (1994), é que a diferença entre a probabilidade assintótica de rejeição para a estatística $\xi_{r v}$, para um $c$ fixo, e a probabilidade empírica de rejeição, obtida através de simulações, é em geral desprezível para $n \geq 50$.

A fim de ilustrar uma aplicação do lema de Wolak, considere um modelo linear generalizado com parte sistemática dada por $\eta=\beta_{1}+\beta_{2} x_{2}+\beta_{3} x_{3}$ e que o interesse é testar $H_{1}: \boldsymbol{\beta} \geq(1,1,1)^{T}$ versus $H_{2}-H_{1}$, onde $H_{2}: \boldsymbol{\beta} \in \mathbb{R}^{3}$. De acordo com os conjuntos definidos para o enunciado do Lema 3.1, o conjunto onde está a situação 
menos favorável é dado por $B=\left\{(1,1,1)^{T}\right\} \cup B_{1} \cup B_{2} \cup B_{3}$, onde

$$
\begin{aligned}
& B_{1}=\left\{\beta_{1}>1, \beta_{2}=1, \beta_{3}=1\right\}, \\
& B_{2}=\left\{\beta_{1}=1, \beta_{2}>1, \beta_{3}=1\right\} \mathrm{e} \\
& B_{3}=\left\{\beta_{1}=1, \beta_{2}=1, \beta_{3}>1\right\} .
\end{aligned}
$$

Isso quer dizer que a distribuição nula menos favorável deve ocorrer para $m=2$ ou $m=3$. No caso de $m=2$, deve-se varrer os três subconjuntos $B_{1}, B_{2}$ e $B_{3}$ para verificar em qual deles está o conjunto de valores menos favorável. Uma solução mais simples, uma vez definido o valor de $m$, é avaliar a quantidade $\Omega_{b}(\boldsymbol{\beta})$ em $\hat{\boldsymbol{\beta}}$. Paula e Sen (1995) e Paula (1999) mostram para certos modelos lineares generalizados, que a matriz $\Omega=R \Sigma^{-1}(\boldsymbol{\beta}) R^{T}$ não depende dos parâmetros sob $\boldsymbol{R} \boldsymbol{\beta}=\boldsymbol{r}$ e para algumas estruturas de $\boldsymbol{R}$. Isso garante que a situação menos favorável seja sempre alcançada quando $\boldsymbol{R} \boldsymbol{\beta}=\boldsymbol{r}$ e que a distribuição nula assintótica das estatísticas do teste seja única.

As estatísticas de Wald e escore, que são assintoticamente equivalentes à estatística da razão de verossimilhança, ficam aqui expressas nas formas abaixo

$$
\begin{aligned}
\xi_{w}= & (\boldsymbol{R} \hat{\boldsymbol{\beta}}-\boldsymbol{R} \tilde{\boldsymbol{\beta}})^{T}\{\hat{\operatorname{Var}}(\boldsymbol{R} \hat{\boldsymbol{\beta}})\}^{-1}(\boldsymbol{R} \hat{\boldsymbol{\beta}}-\boldsymbol{R} \tilde{\boldsymbol{\beta}}) \\
= & \phi(\boldsymbol{R} \hat{\boldsymbol{\beta}}-\boldsymbol{R} \tilde{\boldsymbol{\beta}})^{T}\left\{\boldsymbol{R}\left(\boldsymbol{X}^{T} \hat{\boldsymbol{W}} \boldsymbol{X}\right)^{-1} \boldsymbol{R}^{T}\right\}^{-1}(\boldsymbol{R} \hat{\boldsymbol{\beta}}-\boldsymbol{R} \tilde{\boldsymbol{\beta}}) \mathrm{e} \\
\xi_{s r}= & \{\mathrm{U}(\hat{\boldsymbol{\beta}})-\mathrm{U}(\tilde{\boldsymbol{\beta}})\}^{T} \tilde{\operatorname{V}} \operatorname{ar}(\hat{\boldsymbol{\beta}})\{\mathrm{U}(\hat{\boldsymbol{\beta}})-\mathrm{U}(\tilde{\boldsymbol{\beta}})\} \\
= & \phi^{-1} \mathrm{U}(\tilde{\boldsymbol{\beta}})^{T}\left(\boldsymbol{X}^{T} \tilde{\boldsymbol{W}} \boldsymbol{X}\right)^{-1} \mathbf{U}(\tilde{\boldsymbol{\beta}}),
\end{aligned}
$$

respectivamente, onde $\tilde{\boldsymbol{W}}$ é avaliado em $\tilde{\boldsymbol{\beta}}$. Como ilustração, suponha que a distribuição nula menos favorável seja alcançada quando todas as desigualdades lineares são satisfeitas na forma de igualdades lineares. Então, para grandes amostras e $c>0$, a distribuição nula menos favorável de $\xi_{w}$ fica aproximadamente dada por

$$
\begin{aligned}
& P\left(\xi_{w} \geq c\right)=\sum_{\ell=1}^{k} w(k, k-\ell ; \hat{\Omega}) P\left(\chi_{\ell}^{2} \geq c\right) \mathrm{e} \\
& P\left(\xi_{w}=0\right)=w(k, k ; \hat{\Omega}),
\end{aligned}
$$


onde $\hat{\Omega}=n \boldsymbol{R}\left(\boldsymbol{X}^{T} \hat{\boldsymbol{W}} \boldsymbol{X}\right)^{-1} \boldsymbol{R}^{T}$. Todos os resultados dessa seção podem ser estendidos para o caso em que o parâmetro $\phi$ é desconhecido.

\subsection{Equações de estimação generalizadas}

Em muitas situações práticas envolvendo dados longitudinais, o interesse principal está na forma em que a variável resposta depende das covariáveis. Nesse caso, a correlação entre as observações feitas sobre a mesma unidade experimental pode ser vista como uma perturbação. Com apenas uma observação por unidade experimental, os modelos lineares generalizados descritos no início deste capítulo podem ser usados para descrever essa relação entre a variável resposta e as covariáveis. Com duas ou mais observações repetidas sobre a mesma unidade experimental, a correlação entre essas observações devem ser consideradas na análise. Liang e Zeger (1986) apresentam uma extensão dos modelos lineares generalizados para a análise de dados longitudinais.

Sejam $\boldsymbol{y}_{i}=\left(y_{i 1}, y_{i 2}, \ldots, y_{i t_{i}}\right)^{T}$ um vetor $\left(t_{i} \times 1\right)$ de respostas e $\boldsymbol{X}_{i}=\left(\boldsymbol{x}_{i 1}, \boldsymbol{x}_{i 2}, \ldots, \boldsymbol{x}_{i t_{i}}\right)^{T}$ a matriz $\left(t_{i} \times p\right)$ de valores das covariáveis para a $i$-ésima unidade experimental, $i=1,2, \ldots, n$. Vamos assumir que a densidade marginal de $y_{i j}$ pertence à família exponencial, isto é, sua densidade (ou função de probabilidade) é dada por

$$
f\left(y_{i j} ; \theta_{i j}, \phi\right)=\exp \left\{\phi\left[y_{i j} \theta_{i j}-b\left(\theta_{i j}\right)\right]+c\left(y_{i j}, \phi\right)\right\}
$$

onde $\mathrm{E}\left(y_{i j}\right)=\mu_{i j}=b^{\prime}\left(\theta_{i j}\right), \operatorname{Var}\left(y_{i j}\right)=\phi^{-1} b^{\prime \prime}\left(\theta_{i j}\right)$ e $g\left(\mu_{i j}\right)=\eta_{i j}=x_{i j}^{T} \boldsymbol{\beta}$.

Sem perda de generalidade e para simplificar a notação podemos fazer $t_{i}=t$. Suponha, por enquanto, que as observações feitas sobre a mesma unidade experimental são 
independentes, isto é, a densidade de $\boldsymbol{y}_{i}$ é dada por

$$
h\left(\boldsymbol{y}_{i} ; \boldsymbol{\theta}_{i}, \phi\right)=\prod_{j=1}^{t} f\left(y_{i j} ; \theta_{i j}, o\right)
$$

onde $\boldsymbol{\theta}_{i}=\left(\theta_{i 1}, \theta_{i 2}, \ldots \theta_{i t}\right)$. Se $\boldsymbol{y}$ denota o vetor de respostas $\left(\boldsymbol{y}_{1}, \boldsymbol{y}_{2}, \ldots, \boldsymbol{y}_{n}\right)^{T}$, temos que a log verossimilhança fica dada por

$$
L(\boldsymbol{\beta} ; \boldsymbol{y})=\sum_{i=1}^{n} \sum_{j=1}^{t}\left\{\phi\left[y_{i j} \theta_{i j}-b\left(\theta_{i j}\right)\right]+c\left(y_{i j}, \phi\right)\right\} .
$$

A função escore fica então dada por

$$
\mathrm{U}(\boldsymbol{\beta})=\frac{\partial}{\partial \boldsymbol{\beta}} L(\boldsymbol{\beta} ; \boldsymbol{y})=\phi \sum_{i=1}^{n} \sum_{j=1}^{t}\left\{y_{i j}-b^{\prime}\left(\theta_{i j}\right)\right\} \frac{\partial \theta_{i j}}{\partial \eta_{i j}} x_{i j} .
$$

Definindo a matriz $(t \times t) \boldsymbol{\Delta}_{i}=\operatorname{diag}\left\{\partial \theta_{i j} / \partial \eta_{i j}\right\}, j=1,2, \ldots, t$, podemos escrever $\mathbf{U}(\boldsymbol{\beta})$ como

$$
\mathrm{U}(\boldsymbol{\beta})=\phi \sum_{i=1}^{n} \boldsymbol{X}_{i}^{T} \Delta_{i}\left(\boldsymbol{y}_{i}-\mu_{i}\right) .
$$

O estimador de máxima verossimilhança de $\boldsymbol{\beta}$ que nesse caso será denotado por $\hat{\boldsymbol{\beta}}_{I}$, é a solução da equação $\mathbf{U}\left(\hat{\boldsymbol{\beta}}_{I}\right)=0$. Definindo para cada $i$ a matriz $(t \times t) \mathbf{A}_{i}=$ $\operatorname{diag}\left\{b^{\prime \prime}\left(\theta_{i j}\right)\right\}, j=1,2, \ldots, t$ e sob condições de regularidade, temos o seguinte teorema:

Teorema 3.1. O estimador $\hat{\boldsymbol{\beta}}_{I}$ de $\boldsymbol{\beta}$, é consistente $e \sqrt{n}\left(\hat{\boldsymbol{\beta}}_{I}-\boldsymbol{\beta}\right) \stackrel{\mathcal{D}}{\longrightarrow} \mathrm{N}\left(\mathbf{0}, \boldsymbol{\Sigma}_{I}^{-1}\right)$, quando $n \rightarrow \infty$, onde

$$
\begin{aligned}
\Sigma_{I}^{-1} & =\lim _{n \rightarrow \infty} n\left(\sum_{i=1}^{n} \boldsymbol{X}_{i}^{T} \Delta_{i} \mathbf{A}_{i} \Delta_{i} \boldsymbol{X}_{i}\right)^{-1} \times\left(\sum_{i=1}^{n} \boldsymbol{X}_{i}^{T} \Delta_{i} \operatorname{Cov}\left(\boldsymbol{y}_{i}\right) \Delta_{i} \boldsymbol{X}_{i}\right) \times \\
& \times\left(\sum_{i=1}^{n} \boldsymbol{X}_{i}^{T} \Delta_{i} \mathbf{A}_{i} \Delta_{i} \boldsymbol{X}_{i}\right)^{-1}, \text { ou seja }, \\
\boldsymbol{\Sigma}_{I}^{-1} & =\lim _{n \rightarrow \infty} n\left\{\mathbf{V}_{1}(\boldsymbol{\beta})\right\}^{-1} \mathbf{V}_{2}(\boldsymbol{\beta})\left\{\mathbf{V}_{1}(\boldsymbol{\beta})\right\}^{-1} .
\end{aligned}
$$

Prova. Observe que

$$
\mathrm{E}\{\mathrm{U}(\boldsymbol{\beta})\}=\phi \sum_{i=1}^{n} \boldsymbol{X}_{i}^{T} \Delta_{i} \mathrm{E}\left(\boldsymbol{y}_{i}-\boldsymbol{\mu}_{i}\right)=0
$$


e pelo Teorema Central do Limite temos que

$$
\frac{1}{\sqrt{n}} \mathrm{U}(\boldsymbol{\beta}) \stackrel{\mathcal{D}}{\longrightarrow} \mathrm{N}_{p}\{\mathbf{0}, \mathbf{V}(\boldsymbol{\beta})\}
$$

onde

$$
\begin{aligned}
\mathbf{V}(\boldsymbol{\beta}) & =\lim _{n \rightarrow \infty} \frac{\phi^{2}}{n} \mathrm{E}\left\{\mathrm{U}(\boldsymbol{\beta}) \mathrm{U}(\boldsymbol{\beta})^{T}\right\} \\
& =\lim _{n \rightarrow \infty} \frac{\phi^{2}}{n} \sum_{i=1}^{n} \boldsymbol{X}_{i}^{T} \Delta_{i} \mathrm{E}\left\{\left(\boldsymbol{y}_{i}-\boldsymbol{\mu}_{i}\right)\left(\boldsymbol{y}_{i}-\boldsymbol{\mu}_{i}\right)^{T}\right\} \Delta_{i} \boldsymbol{X}_{i} \\
& =\lim _{n \rightarrow \infty} \frac{\phi^{2}}{n} \sum_{i=1}^{n} \boldsymbol{X}_{i}^{T} \Delta_{i} \operatorname{Cov}\left(\boldsymbol{y}_{i}\right) \Delta_{i} \boldsymbol{X}_{i} .
\end{aligned}
$$

Por outro lado,

$$
\mathrm{E}\left\{\frac{\partial}{\partial \boldsymbol{\beta}} \mathrm{U}(\boldsymbol{\beta})\right\}=\sum_{i=1}^{n} \boldsymbol{X}_{i}^{T} \boldsymbol{\Delta}_{i} \boldsymbol{A}_{i} \Delta_{i} \boldsymbol{X}_{i},
$$

e pela Lei Fraca dos Grandes Números de Khintchine,

$$
\frac{1}{n} \frac{\partial}{\partial \boldsymbol{\beta}} \mathrm{U}(\boldsymbol{\beta})+\frac{\phi}{n} \sum_{i=1}^{n} \boldsymbol{X}_{i}^{T} \Delta_{i} \boldsymbol{A}_{i} \Delta_{i} \boldsymbol{X}_{i} \stackrel{P}{\longrightarrow} 0
$$

Expandindo em série de Taylor a função $\mathbf{U}\left(\hat{\boldsymbol{\beta}}_{I}\right)$ em torno de $\boldsymbol{\beta}$ temos que

$$
\mathbf{U}\left(\hat{\boldsymbol{\beta}}_{I}\right)=\mathbf{U}(\boldsymbol{\beta})+\frac{\partial}{\partial \boldsymbol{\beta}} \mathbf{U}(\boldsymbol{\beta})\left(\hat{\boldsymbol{\beta}}_{I}-\boldsymbol{\beta}\right)+O_{p}(1)
$$

ou seja,

$$
\hat{\boldsymbol{\beta}}_{I}-\boldsymbol{\beta}=\left\{-\frac{\partial}{\partial \boldsymbol{\beta}} \mathrm{U}(\boldsymbol{\beta})\right\}^{-1} \mathrm{U}(\boldsymbol{\beta})+o_{p}\left(n^{-1 / 2}\right),
$$

e pela Lei Fraca dos Grandes Números de Khintchine temos que $\hat{\boldsymbol{\beta}}_{I}-\boldsymbol{\beta} \stackrel{P}{\rightarrow} \mathbf{0}$, ou seja $\hat{\boldsymbol{\beta}}_{I}$ é consistente.

Agora, dividindo (3.5.2) por $\sqrt{n}$ temos que

$$
\mathbf{0}=\frac{\mathbf{U}(\boldsymbol{\beta})}{\sqrt{n}}+\frac{1}{n} \frac{\partial}{\partial \boldsymbol{\beta}} \mathbf{U}(\boldsymbol{\beta}) \sqrt{n}\left(\hat{\boldsymbol{\beta}}_{I}-\boldsymbol{\beta}\right)+O_{p}\left(n^{-1 / 2}\right),
$$

ou ainda

$$
\sqrt{n}\left(\hat{\boldsymbol{\beta}}_{I}-\boldsymbol{\beta}\right)=\left\{-\frac{1}{n} \frac{\partial}{\partial \boldsymbol{\beta}} \mathbf{U}(\boldsymbol{\beta})\right\}^{-1} \frac{\mathbf{U}(\boldsymbol{\beta})}{\sqrt{n}}+o_{p}\left(n^{-1 / 2}\right),
$$

portanto, pelo Teorema de Slutsky, segue o resultado do teorema. 
A variância de $\hat{\boldsymbol{\beta}}_{I}$ nesse teorema pode ser consistentemente estimada por

$$
\hat{\boldsymbol{V}}_{\boldsymbol{I}}=\left\{\mathrm{V}_{1}\left(\hat{\boldsymbol{\beta}}_{I}\right)\right\}^{-1}\left[\sum_{i=1}^{n} \boldsymbol{X}_{i} \boldsymbol{\Delta}_{i}\left(\boldsymbol{y}_{i}-\boldsymbol{\mu}_{i}\right)\left(\boldsymbol{y}_{i}-\boldsymbol{\mu}_{i}\right)^{T} \Delta_{i} \boldsymbol{X}_{i}\right]_{\hat{\boldsymbol{\beta}}_{I}}\left\{\mathrm{~V}_{1}\left(\hat{\boldsymbol{\beta}}_{I}\right)\right\}^{-1}
$$

Note que nesse caso não é necessário estimar $\phi$ para se obter a estimativa $\hat{V}_{I}$.

O estimador $\hat{\boldsymbol{\beta}}_{I}$ obtido sob a hipótese de independência, perde eficiência quando existe correlação entre as observações feitas sobre a mesma unidade experimental. Com a finalidade de aumentar a eficiência, Liang e Zeger (1986) apresentam as Equações de Estimação Generalizadas que levam em consideração a correlação entre as observações de uma mesma unidade experimental. O estimador de $\boldsymbol{\beta}$ obtido das EEGs será denotado $\operatorname{por} \hat{\boldsymbol{\beta}}_{G}$.

Sejam $\Gamma(\alpha)$ uma matriz simétrica $(t \times t)$ satisfazendo as condições para ser uma matriz de correlação e $\boldsymbol{\alpha}$ um vetor $(q \times 1)$ que caracteriza completamente $\Gamma(\boldsymbol{\alpha})$. A matriz $\Gamma(\boldsymbol{\alpha})$ é chamada Matriz de Correlação de Trabalho. Definindo

$$
\Omega_{i}=\phi^{-1} \mathbf{A}_{i}^{1 / 2} \Gamma(\alpha) \mathbf{A}_{i}^{1 / 2}
$$

e sendo

$$
\boldsymbol{D}_{i}=\frac{\partial}{\partial \boldsymbol{\beta}}\left\{b^{\prime}\left(\theta_{i j}\right)\right\}=\frac{\partial b^{\prime}\left(\theta_{i j}\right)}{\partial \theta_{i j}} \frac{\partial \theta_{i j}}{\partial \eta_{i j}} \frac{\partial \eta_{i j}}{\partial \boldsymbol{\beta}}=\mathbf{A}_{i} \Delta_{i} \boldsymbol{X}_{i},
$$

as Equações de Estimação Generalizadas são definidas por $\mathbf{U}\left(\hat{\boldsymbol{\beta}}_{G} \mid \hat{\alpha}, \hat{\phi}\right)=0$, onde

$$
\mathrm{U}(\boldsymbol{\beta} \mid \boldsymbol{\alpha}, \phi)=\sum_{i=1}^{n} \boldsymbol{D}_{i}^{T} \boldsymbol{\Omega}_{i}^{-1}\left(\boldsymbol{y}_{i}-\boldsymbol{\mu}_{i}\right)
$$

e $\hat{\alpha}$ e $\hat{\phi}$ são estimadores consistentes de $\alpha$ e $\phi$, respectivamente. Observe que sendo $\mathbf{A}_{i}=\operatorname{diag}\left\{b^{\prime \prime}\left(\theta_{i j}\right)\right\}=\operatorname{diag}\left\{\phi \operatorname{Var}\left(y_{i j}\right)\right\}$, então $\boldsymbol{\Omega}_{i}$ seria a verdadeira covariância de $\boldsymbol{y}_{i}$ se $\boldsymbol{\Gamma}(\boldsymbol{\alpha})$ fosse a verdadeira matriz de correlação. A equação (3.5.4) reduz-se ao caso de independência se a matriz $\Gamma(\alpha)$ é a identidade. Note também que para cada $i, \mathbf{U}_{i}(\boldsymbol{\beta} \mid \boldsymbol{\alpha}, \phi)=\boldsymbol{D}_{i}^{T} \boldsymbol{\Omega}_{i}^{-1}\left(\boldsymbol{y}_{i}-\boldsymbol{\mu}_{i}\right)$ é semelhante à função de quase-verossimilhança 
(Wedderburn, 1974). Nesse caso. entretanto, $\boldsymbol{\Omega}_{i}$ depende tanto de $\boldsymbol{\beta}$ como de $\boldsymbol{\alpha}$. A equação (3.5.4) pode ser escrita em função de $\hat{\boldsymbol{\beta}}_{G}$ somente, substituindo-se $\boldsymbol{\alpha}$ por um estimador $n^{1 / 2}$-consistente $\hat{\boldsymbol{\alpha}}(\boldsymbol{\beta}, \phi)$, quando $\boldsymbol{\beta}$ e $\phi$ são conhecidos, isto é, tal que $n^{1 / 2}\{\hat{\boldsymbol{\alpha}}(\boldsymbol{\beta}, \phi)-\boldsymbol{\alpha}\}=O_{p}(1)$ e $\phi$ por um estimador $n^{1 / 2}$-consistente $\hat{\phi}(\boldsymbol{\beta})$, quando $\boldsymbol{\beta}$ é conhecido. Com isso, obtém-se

$$
\mathrm{U}\left(\hat{\boldsymbol{\beta}}_{G} \mid \hat{\boldsymbol{\alpha}}, \hat{\phi}\right)=\sum_{i=1}^{n} \mathbf{U}_{i}\left\{\hat{\boldsymbol{\beta}}_{G}, \hat{\boldsymbol{\alpha}}\left[\hat{\boldsymbol{\beta}}_{G}, \hat{\phi}\left(\hat{\boldsymbol{\beta}}_{G}\right)\right]\right\}=0 .
$$

O estimador $\hat{\boldsymbol{\beta}}_{G}$ definido como sendo a solução da equação (3.5.5), tem propriedades definidas pelo seguinte teorema:

Teorema 3.2. Sob condições de regularidade e dado que

1. $\hat{\boldsymbol{\alpha}}$ é $n^{1 / 2}$-consistente dados $\boldsymbol{\beta}$ e $\phi$;

2. $\hat{\phi} e ́ n^{1 / 2}$-consistente dado $\boldsymbol{\beta}$ e;

3. $\left|\frac{\partial}{\partial \phi} \hat{\boldsymbol{\alpha}}(\boldsymbol{\beta}, \phi)\right| \leq H(\boldsymbol{\beta} ; \boldsymbol{y})=O_{p}(1)$,

então $n^{1 / 2}\left(\hat{\boldsymbol{\beta}}_{G}-\boldsymbol{\beta}\right)$ é assintoticamente normal multivariada com média zero e matriz de covariância $\Sigma^{-1}$ dada por

$$
\boldsymbol{\Sigma}^{-1}=\lim _{n \rightarrow \infty} n\left(\sum_{i=1}^{n} \boldsymbol{D}_{i}^{T} \boldsymbol{\Omega}_{i}^{-1} \boldsymbol{D}_{i}\right)^{-1}\left\{\sum_{i=1}^{n} \boldsymbol{D}_{i}^{T} \boldsymbol{\Omega}_{i}^{-1} \operatorname{Cov}\left(\boldsymbol{y}_{i}\right) \boldsymbol{\Omega}_{i}^{-1} \boldsymbol{D}_{i}\right\}\left(\sum_{i=1}^{n} \boldsymbol{D}_{i}^{T} \boldsymbol{\Omega}_{i}^{-1} \boldsymbol{D}_{i}\right)^{-1} .
$$

Prova. Esse teorema é um caso particular do Teorema 6 de Artes (1998). Expandindose em série de Taylor a função $U\left(\hat{\boldsymbol{\beta}}_{G} \mid \hat{\boldsymbol{\alpha}}, \hat{\phi}\right)$ em torno de $\boldsymbol{\beta}$ temos que

$$
U\left(\hat{\boldsymbol{\beta}}_{G} \mid \hat{\boldsymbol{\alpha}}, \hat{\phi}\right)=U(\boldsymbol{\beta} \mid \hat{\boldsymbol{\alpha}}, \hat{\phi})+\frac{\partial}{\partial \boldsymbol{\beta}} U(\boldsymbol{\beta} \mid \hat{\boldsymbol{\alpha}}, \hat{\phi})\left(\hat{\boldsymbol{\beta}}_{G}-\boldsymbol{\beta}\right)+O_{p}(1),
$$

dividindo essa expressão por $\sqrt{n}$ vem que

$$
\sqrt{n}\left(\hat{\boldsymbol{\beta}}_{G}-\boldsymbol{\beta}\right)=\left\{-\frac{1}{n} \frac{\partial}{\partial \boldsymbol{\beta}} \mathbf{U}(\boldsymbol{\beta} \mid \hat{\boldsymbol{\alpha}}, \hat{\phi})\right\}^{-1}\left\{\frac{1}{\sqrt{n}} \mathbf{U}(\boldsymbol{\beta} \mid \hat{\boldsymbol{\alpha}}, \hat{\phi})\right\}+o_{p}(1) .
$$


Pelos Lemas 5 e 7 respectivamente (dados em Artes, 1998, Capítulo 3) temos que

$$
\frac{1}{\sqrt{n}} \mathrm{U}(\boldsymbol{\beta} \mid \hat{\boldsymbol{\alpha}}, \hat{\phi}) \stackrel{\mathcal{D}}{\longrightarrow} \mathrm{N}_{p}(\mathbf{0}, \mathrm{G})
$$

onde $\mathbf{G}=\lim _{n \rightarrow \infty} \frac{1}{n} \sum_{i=1}^{n} \boldsymbol{D}_{i}^{T} \boldsymbol{\Omega}_{i}^{-1} \operatorname{Cov}\left(\boldsymbol{y}_{i}\right) \boldsymbol{\Omega}_{i}^{-1} \boldsymbol{D}_{i}$ e

$$
\frac{1}{n} \frac{\partial}{\partial \boldsymbol{\beta}} \mathrm{U}(\boldsymbol{\beta} \mid \hat{\boldsymbol{\alpha}}, \hat{\phi})+\frac{1}{n} \sum_{i=1}^{n} \boldsymbol{D}_{i}^{T} \boldsymbol{\Omega}_{i}^{-1} \boldsymbol{D}_{i} \stackrel{P}{\longrightarrow} 0,
$$

e pelo Teorema de Slutsky segue-se o resultado.

Se a dependência para cada unidade experimental foi especificada corretamente, então uma estimativa consistente para $\operatorname{Var}\left(\hat{\boldsymbol{\beta}}_{G}\right)$ é dada por $H_{1}^{-1}\left(\hat{\boldsymbol{\beta}}_{G}\right)$, onde

$$
H_{1}\left(\hat{\boldsymbol{\beta}}_{G}\right)=\sum_{i=1}^{n} \hat{\boldsymbol{D}}_{i} \hat{\boldsymbol{\Omega}}_{i}^{-1} \hat{\boldsymbol{D}}_{i}
$$

com $\hat{\boldsymbol{D}}_{i}$ sendo avaliado em $\hat{\boldsymbol{\beta}}_{G}$ enquanto $\hat{\boldsymbol{\Omega}}_{i}$ é avaliado em $\left(\hat{\phi}, \hat{\boldsymbol{\alpha}}, \hat{\boldsymbol{\beta}}_{G}\right)$. Entretanto, se a matriz de correlação de trabalho $\Gamma(\boldsymbol{\alpha})$ é definida incorretamente, a estimativa $H_{1}^{-1}\left(\hat{\boldsymbol{\beta}}_{G}\right)$ pode ser inconsistente. Uma estimativa robusta para $\operatorname{Var}\left(\hat{\boldsymbol{\beta}}_{G}\right)$, proposta por Liang e Zeger (1986), é dada por $\hat{\boldsymbol{V}}_{G}=H_{1}^{-1}\left(\hat{\boldsymbol{\beta}}_{G}\right) H_{2}\left(\hat{\boldsymbol{\beta}}_{G}\right) H_{1}^{-1}\left(\hat{\boldsymbol{\beta}}_{G}\right)$, onde

$$
H_{2}\left(\hat{\boldsymbol{\beta}}_{G}\right)=\sum_{i=1}^{n}\left\{\hat{\boldsymbol{D}}_{i}^{T} \hat{\Omega}_{i}^{-1}\left(\boldsymbol{y}_{i}-\hat{\boldsymbol{\mu}}_{i}\right)\left(\boldsymbol{y}_{i}-\hat{\boldsymbol{\mu}}_{i}\right)^{T} \hat{\Omega}_{i}^{-1} \hat{\boldsymbol{D}}_{i}\right\}
$$

A estimativa $\hat{V}_{G}$ é consistente mesmo quando a matriz de correlação de trabalho não corresponde a $\operatorname{Cov}\left(\boldsymbol{y}_{i}\right)$. A consistência de $\hat{\boldsymbol{\beta}}_{G}$ e $\hat{\boldsymbol{V}}_{G}$ depende somente da especificação correta da média $\mathrm{E}\left(\boldsymbol{y}_{i}\right)$ e não da escolha de $\boldsymbol{\Gamma}(\boldsymbol{\alpha})$. A estimativa $\hat{\boldsymbol{V}}_{G}$ não depende da escolha dos estimadores de $\alpha$ e $\phi$ dentre aqueles que são $n^{1 / 2}$-consistente. O processo iterativo para calcular $\hat{\boldsymbol{\beta}}_{G}$ combina o método scoring de Fisher para $\boldsymbol{\beta}$ com o método de momentos para $\alpha$ e $\phi$. Dadas as correntes estimativas $\hat{\alpha}$ e $\hat{\phi}$, o processo iterativo sugerido por Liang e Zeger (1986) é dado por

$$
\begin{aligned}
\hat{\boldsymbol{\beta}}^{(m+1)} & =\hat{\boldsymbol{\beta}}^{(m)}-\left\{\sum_{i=1}^{n} \boldsymbol{D}_{i}^{T}\left(\hat{\boldsymbol{\beta}}^{(m)}\right) \tilde{\boldsymbol{\Omega}}_{i}^{-1}\left(\hat{\boldsymbol{\beta}}^{(m)}\right) \boldsymbol{D}_{i}\left(\hat{\boldsymbol{\beta}}^{(m)}\right)\right\}^{-1} \times \\
& \times\left\{\sum_{i=1}^{n} \boldsymbol{D}_{i}^{T}\left(\hat{\boldsymbol{\beta}}^{(m)}\right) \tilde{\boldsymbol{\Omega}}_{i}^{-1}\left(\hat{\boldsymbol{\beta}}^{(m)}\right)\left[\boldsymbol{y}_{i}-\boldsymbol{\mu}_{i}\left(\hat{\boldsymbol{\beta}}^{(m)}\right)\right]\right\},
\end{aligned}
$$


onde $\tilde{\Omega}_{i}(\boldsymbol{\beta})=\tilde{\Omega}_{i}\{\boldsymbol{\beta}, \hat{\alpha}[\boldsymbol{\beta}, \hat{\phi}(\boldsymbol{\beta})]\}$. Em cada passo do processo iterativo, $\boldsymbol{\alpha}$ e $\phi$ podem ser estimados do corrente resíduo de Pearson

$$
\hat{r}_{i j}=\left\{y_{i j}-b^{\prime}\left(\hat{\theta}_{i j}\right)\right\} / \sqrt{b^{\prime \prime}\left(\hat{\theta}_{i j}\right)},
$$

onde $\hat{\theta}_{i j}$ depende da corrente estimativa de $\boldsymbol{\beta}$. A estimativa de $\phi$ é dada por

$$
\hat{\phi}=\sum_{i=1}^{n} \hat{r}_{i j}^{2} /(N-p),
$$

onde $N=\sum_{i=1}^{n} t_{i}$. A estimativa de $\boldsymbol{\alpha}$ depende da escolha de $\Gamma(\boldsymbol{\alpha})$, em geral $\boldsymbol{\alpha}$ é uma função simples de

$$
\hat{\Gamma}_{j \ell}=\sum_{i=1}^{n} \hat{r}_{i j} \hat{r}_{i \ell} /(N-p) .
$$

O procedimento das EEGs para estimar $\boldsymbol{\beta}$ permite que a estrutura de correlação entre as observações da mesma unidade experimental seja especificada de diferentes formas. Apresentamos a seguir algumas especificações usadas para $\Gamma(\boldsymbol{\alpha})$.

1. Quando a matriz de correlação $\Gamma(\boldsymbol{\alpha})$ é a identidade, obtém-se o caso de independência como foi visto no início desta seção. Entretando, para qualquer $\Gamma(\boldsymbol{\alpha})$ dada, $\hat{\boldsymbol{\beta}}_{G}$ e $\hat{\mathbf{V}}_{G}$ serão consistentes e a eficiência cresce quanto mais próxima da verdadeira matriz de correlação estiver a escolha de $\Gamma(\boldsymbol{\alpha})$.

2. A estrutura de correlação simétrica assume que $\operatorname{Corr}\left(y_{i j}, y_{i k}\right)=\alpha, \forall j \neq k$. Essa é a estrutura usada nos modelos de efeitos aleatórios (veja, por exemplo, Laird e Ware, 1982). Nesse caso, dado $\phi$, um estimador de $\alpha$ é dado por

$$
\hat{\alpha}=\phi \frac{\sum_{i=1}^{n} \sum_{j>k} \hat{r}_{i j} \hat{r}_{i k}}{\left\{\sum_{i=1}^{n} \frac{1}{2} t_{i}\left(t_{i}-1\right)-p\right\}} .
$$

3. A estrutura de correlação que especifica $\operatorname{Corr}\left(y_{i j}, y_{i(j+1)}\right)=\alpha_{j}, j=1,2, \ldots, t_{i}-1$, é equivalente ao modelo 1-dependente e um estimador natural de $\alpha_{j}$ dados $\beta$ e $\phi$ 
é dado por

$$
\hat{\alpha}_{j}=\phi \frac{1}{n-p} \sum_{i=1}^{n} \hat{r}_{i j} \hat{r}_{i(j+1)} .
$$

Como um caso especial, o modelo $\mathrm{AR}(1)$ especifica $\alpha_{j}=\alpha, j=1,2, \ldots, t_{i}-1 \mathrm{e}$ um estimador de $\alpha$ é dado por

$$
\hat{\alpha}=\frac{1}{t_{i}-1} \sum_{j=1}^{t_{i}-1} \hat{\alpha}_{j}
$$

4. Quando $\Gamma(\alpha)$ é totalmente não estruturada, a mesma pode ser estimada por

$$
\hat{\Gamma}=\frac{\phi}{n} \sum_{i=1}^{n} \mathbf{A}_{i}^{-1 / 2}\left(\boldsymbol{y}_{i}-\boldsymbol{\mu}_{i}\right)\left(\boldsymbol{y}_{i}-\boldsymbol{\mu}_{i}\right)^{T} \mathbf{A}_{i}^{-1 / 2}
$$

Em nenhum dos casos acima é necessário estimar $\phi$ para obter $\hat{\boldsymbol{\beta}}_{G}$ e $\operatorname{Var}\left(\hat{\boldsymbol{\beta}}_{G}\right)$.

\subsection{1 g Grupos}

Vamos supor, sem perda de generalidade, que temos agora $g$ grupos tal que cada um tem $n_{\ell}$ unidades experimentais as quais são observadas nas mesmas $t$ ocasiões. As respostas observadas para a $i$-ésima unidade experimental do $\ell$-ésimo grupo forma um vetor $(t \times 1)$ dado por $\boldsymbol{y}_{i \ell}=\left(y_{i \ell 1}, y_{i \ell 2}, \ldots, y_{i \ell t}\right)^{T}, i=1,2, \ldots, n_{\ell}$ e $\ell=1,2, \ldots, g$. Ademais, assumiremos que $\mathrm{E}\left(y_{i \ell j}\right)=\mu_{\ell}$ e que $\operatorname{Var}\left(y_{i \ell j}\right)=\phi^{-1} V_{\ell}$. Como temos $g$ grupos independentes o interesse principal está em estimar as médias $\mu_{1}, \mu_{2}, \ldots, \mu_{g}$. Seguindo a notação da seção anterior podemos definir uma EEG para cada média $\mu_{\ell}$, dada por $U\left(\hat{\mu}_{\ell} \mid \hat{\alpha}, \hat{\phi}\right)=0$, onde

$$
U\left(\mu_{\ell} \mid \boldsymbol{\alpha}, \phi\right)=\sum_{i=1}^{n_{\ell}} \mathbf{1}_{t}^{T} \boldsymbol{\Gamma}_{i \ell}^{-1}(\alpha)\left(y_{i \ell}-\mu_{\ell} \mathbf{1}_{t}\right)
$$

$\Gamma_{i \ell}(\alpha)$ é a matriz de correlação de trabalho correspondente à $i$-ésima unidade experimental do $\ell$-ésimo grupo e $1_{t}$ é um vetor $(t \times 1)$ de uns. Resolvendo-se $U\left(\hat{\mu}_{\ell} \mid \hat{\boldsymbol{\alpha}}, \hat{\phi}\right)=0$ 
obtém-se a seguinte estimativa para $\mu_{\ell}$ :

$$
\left.\hat{\mu}_{\ell}=\left\{\sum_{i=1}^{n_{\ell}} \mathbf{1}_{t}^{T} \boldsymbol{\Gamma}_{i \ell}^{-1}(\hat{\boldsymbol{\alpha}}) \mathbf{1}_{t}\right)\right\}^{-1}\left\{\sum_{i=1}^{n_{\ell}} \mathbf{1}_{t}^{T} \boldsymbol{\Gamma}_{i \ell}^{-1}(\hat{\boldsymbol{\alpha}}) \boldsymbol{y}_{i \ell}\right\} .
$$

Conforme $n_{\ell} \rightarrow \infty, \forall \ell$, sob algumas condições suaves de regularidade segue que

$$
\sqrt{n}(\hat{\mu}-\mu) \stackrel{\mathcal{D}}{\longrightarrow} N_{g}\left(0, \Sigma_{\mu}^{-1}\right)
$$

onde $n=\sum_{\ell=1}^{g} n_{\ell}, \boldsymbol{\mu}=\left(\mu_{1}, \mu_{2}, \ldots, \mu_{g}\right)^{T}$ e $\Sigma_{\mu}^{-1}=\operatorname{diag}\left\{\sigma_{1}^{2}, \sigma_{2}^{2}, \ldots, \sigma_{g}^{2}\right\}$, com

$$
\sigma_{\ell}^{2}=\lim _{n \rightarrow \infty} n\left\{\sum_{i=1}^{n_{\ell}} \mathbf{1}_{t}^{T} \boldsymbol{\Gamma}_{i \ell}^{-1}(\boldsymbol{\alpha}) \mathbf{1}_{t}\right\}^{-2}\left\{\sum_{i=1}^{n_{\ell}} \mathbf{1}_{t}^{T} \boldsymbol{\Gamma}_{i \ell}^{-1}(\boldsymbol{\alpha}) \operatorname{Cov}\left(\boldsymbol{y}_{i \ell}\right) \boldsymbol{\Gamma}_{i \ell}^{-1}(\boldsymbol{\alpha}) \mathbf{1}_{t}\right\}
$$

Uma estimativa robusta para $\Sigma_{\boldsymbol{\mu}}^{-1}$ é dada por $n \hat{\mathbf{V}}_{\boldsymbol{\mu}}$, onde $\hat{\mathrm{V}}_{\boldsymbol{\mu}}=\operatorname{diag}\left\{\hat{v}_{\mu_{1}}, \hat{v}_{\mu_{2}}, \ldots, \hat{v}_{\mu_{g}}\right\}$, $\hat{v}_{\mu_{\ell}}=H_{1 \ell}^{-2}\left(\hat{\mu}_{\ell}\right) H_{2 \ell}\left(\hat{\mu}_{\ell}\right), \mathrm{com}$

$$
H_{1 \ell}\left(\hat{\mu}_{\ell}\right)=\sum_{i=1}^{n_{\ell}} \mathbf{1}_{t}^{T} \boldsymbol{\Gamma}_{i \ell}^{-1}(\hat{\boldsymbol{\alpha}}) \mathbf{1}_{t} \text { e } H_{2 \ell}\left(\hat{\mu}_{\ell}\right)=\sum_{i=1}^{n_{\ell}}\left\{\mathbf{1}_{t}^{T} \boldsymbol{\Gamma}_{i \ell}^{-1}(\hat{\boldsymbol{\alpha}})\left(\boldsymbol{y}_{i \ell}-\hat{\mu}_{\ell} \mathbf{1}_{t}\right)\right\}^{2}
$$

\subsection{Estatística tipo Wald}

Como na seção anterior, suponha, sem perda de generalidade, que cada uma de $n$ unidades experimentais é observada nas mesmas $t$ oportunidades. O vetor $(t \times 1)$ de respostas da $i$-ésima unidade experimental, $\boldsymbol{y}_{i}=\left(y_{i 1}, y_{i 2}, \ldots, y_{i t}\right)^{T}$, pertence à família exponencial de distribuições tal que $\mathrm{E}\left(y_{i j}\right)=\mu_{i j}$ com preditor linear $\eta_{i j}=\boldsymbol{x}_{i j}^{T} \boldsymbol{\beta}$. O interesse é testar hipóteses sobre o vetor de parâmetros $\boldsymbol{\beta}=\left(\beta_{1}, \beta_{2}, \ldots, \beta_{p}\right)^{T}$.Especificamente, estamos interessados em testar

$$
\begin{array}{ll}
\text { (Caso I) } & H_{0}: \boldsymbol{R} \boldsymbol{\beta}=\boldsymbol{r} \text { versus } H_{1}: \boldsymbol{R} \boldsymbol{\beta} \geq \boldsymbol{r}, \\
(\text { Caso II) } & H_{1}: \boldsymbol{R} \boldsymbol{\beta} \geq \boldsymbol{r} \text { versus } H_{2}-H_{1}
\end{array}
$$


onde $H_{2}: \boldsymbol{\beta} \in \mathbb{R}^{p}$, com pelo menos uma desigualdade estrita em $H_{1}$ no caso I de modo que $H_{0} \subset H_{1} \subset H_{2}, R$ e $\boldsymbol{r}$ são definidos como na Seção 2.3. Uma estatística tipo Wald para testar essas hipóteses, foi proposta por Kodde e Palm (1986) a qual mede a diferença entre as distâncias do estimador não restrito $\hat{\boldsymbol{\beta}}$ e os estimadores restritos pelas hipóteses, na métrica da matriz de covariância de $\hat{\beta}$. Para determinar a distribuição nula assintótica da estatística de Kodde e Palm, que será aqui denotada por $\xi_{k p}$, o estimador não restrito $\hat{\boldsymbol{\beta}}$ deve ser assintoticamente consistente e

$$
\sqrt{n}(\hat{\boldsymbol{\beta}}-\boldsymbol{\beta}) \stackrel{\mathcal{D}}{\longrightarrow} \mathbb{N}_{p}\left(0, \Sigma^{-1}\right)
$$

onde $\Sigma^{-1}$ pode ser consistentemente estimada. Portanto, como foi mostrado na seção anterior, para o caso de dados correlacionados, o estimador não restrito $\hat{\boldsymbol{\beta}}_{G}$ obtido das EEGs, satisfaz as condições para o uso da estatística $\xi_{k p}$ para testar as hipóteses (3.6.1) e (3.6.2).

Sejam, então, $\hat{\boldsymbol{\beta}}_{G}$ o estimador de $\boldsymbol{\beta}$ obtido das EEGs e $\boldsymbol{\Sigma}^{-1}$ a sua matriz de covariância assintótica. Considere a transformação $\gamma=\boldsymbol{R} \boldsymbol{\beta}-\boldsymbol{r}$ e $\hat{\boldsymbol{\gamma}}_{G}=\boldsymbol{R} \hat{\boldsymbol{\beta}}_{G}-\boldsymbol{r}$. Então $\hat{\boldsymbol{\gamma}}_{G}$ é assintoticamente consistente e

$$
\sqrt{n}\left(\hat{\gamma}_{G}-\gamma\right) \stackrel{\mathcal{D}}{\longrightarrow} \mathrm{N}_{k}(\mathbf{0}, \Omega)
$$

conforme $n \rightarrow \infty$, onde $\Omega=R \Sigma^{-1} R^{T}$. Sejam $\bar{\gamma}$ e $\tilde{\gamma}$ os estimadores de $\gamma$ sob as hipóteses $H_{0}$ e $H_{1}$, respectivamente.

Definindo

$$
D_{0}=\left\|\hat{\gamma}_{G}-\bar{\gamma}\right\|=\min _{\gamma}\left\{n\left(\hat{\gamma}_{G}-\gamma\right)^{T} \Omega^{-1}\left(\hat{\gamma}_{G}-\gamma\right) \text { sujeito a } \gamma=0\right\}
$$

$\mathrm{e}$

$$
D_{1}=\left\|\hat{\gamma}_{G}-\tilde{\gamma}\right\|=\min _{\gamma}\left\{n\left(\hat{\gamma}_{G}-\gamma\right)^{T} \Omega^{-1}\left(\hat{\gamma}_{G}-\gamma\right) \text { sujeito a } \gamma \geq 0\right\}
$$


a estatística de Kodde e Palm é dada por

$$
\begin{aligned}
\xi_{k p} & =D_{0}-D_{1} \\
& =\left\|\hat{\gamma}_{G}-\bar{\gamma}\right\|-\left\|\hat{\gamma}_{G}-\tilde{\gamma}\right\| \\
& =\|\tilde{\gamma}\|-\|\bar{\gamma}\| .
\end{aligned}
$$

Como $H_{0} \subset H_{1} \subset H_{2}$, então $\xi_{k p}$ será sempre não negativa. A hipótese nula é rejeitada se $\xi_{k p}$ excede um valor crítico. Para o caso de testar as hipóteses (3.6.1) a estatística de Kodde Palm fica dada por

$$
\xi_{k p}=\|\tilde{\gamma}\|=n \tilde{\gamma}^{T} \Omega^{-1} \tilde{\gamma}
$$

Quando $\Omega$ é conhecida, pelo Teorema 2.2 a distribuição nula assintótica de $\xi_{k p}$, para todo $c>0$, é tal que

$$
\begin{aligned}
\lim _{n \rightarrow \infty} P\left(\xi_{k p} \geq c \mid \Omega\right) & =\sum_{\ell=1}^{k} w(k, \ell ; \Omega) P\left(\chi_{\ell}^{2} \geq c\right) \mathrm{e} \\
\lim _{n \rightarrow \infty} P\left(\xi_{k p}=0 \mid \Omega\right) & =w(k, 0 ; \Omega) .
\end{aligned}
$$

Quando $\Omega=\sigma^{2} \Sigma$ com $\sigma^{2}$ desconhecida e $\boldsymbol{\Sigma}$ conhecida, pelo Corolário 2.1 a distribuição nula assintótica de $\xi_{k p}$, para todo $c>0$, é tal que

$$
\begin{aligned}
& \lim _{n \rightarrow \infty} P\left(\xi_{k p} \geq c \mid \Omega\right)=\sum_{\ell=1}^{k} w(k, \ell ; \Omega) P\left(F_{\ell, n-p} \geq \frac{c}{\ell}\right) \mathrm{e} \\
& \lim _{n \rightarrow \infty} P\left(\xi_{k p}=0 \mid \Omega\right)=w(k, 0 ; \Omega) .
\end{aligned}
$$

Para o caso de testar as hipóteses (3.6.2), a distância $D_{1}$ é nula e nesse caso a estatística de Kodde e Palm fica dada por

$$
\xi_{k p}=\left\|\hat{\gamma}_{G}-\tilde{\gamma}\right\|=n\left(\hat{\gamma}_{G}-\tilde{\gamma}\right)^{T} \Omega^{-1}\left(\hat{\gamma}_{G}-\tilde{\gamma}\right)
$$

Quando $\Omega$ é conhecida, pelo Teorema 2.3 a distribuição nula assintótica menos favorável de $\xi_{k p}$ satisfaz, para todo $c>0$

$$
\begin{aligned}
\lim _{n \rightarrow \infty} P\left(\xi_{k p} \geq c \mid \Omega\right) & =\sum_{\ell=1}^{k} w(k, k-\ell ; \Omega) P\left(\chi_{\ell}^{2} \geq c\right) \mathrm{e} \\
\lim _{n \rightarrow \infty} P\left(\xi_{k p}=0 \mid \Omega\right) & =w(k, k ; \Omega) .
\end{aligned}
$$


Se $\Omega=\sigma^{2} \Sigma$ com $\sigma^{2}$ desconhecida e $\Sigma$ conhecida, pelo Corolário 2.2 a distribuição nula assintótica menos favorável da estatística $\xi_{k p}$ satisfaz, para todo $c>0$

$$
\begin{aligned}
& \lim _{n \rightarrow \infty} P\left(\xi_{k p} \geq c \mid \Omega\right)=\sum_{\ell=1}^{k} w(k, k-\ell ; \Omega) P\left(F_{\ell, n-p} \geq \frac{c}{\ell}\right) \mathrm{e} \\
& \lim _{n \rightarrow \infty} P\left(\xi_{k p}=0 \mid \Omega\right)=w(k, k ; \Omega) .
\end{aligned}
$$

Vamos discutir a seguir como fica a distribuição nula assintótica da estatística $\xi_{k p}$ quando $\Omega$ é desconhecida para os casos I e II.

\section{Caso I}

Uma estimativa consistente para $\Sigma^{-1}$ é dada por $n \hat{\mathbf{V}}_{G}$. Logo, a estatística de Kodde e Palm toma a forma

$$
\begin{aligned}
\xi_{k p} & =n \tilde{\boldsymbol{\gamma}}^{T} \hat{\boldsymbol{\Omega}}^{-1} \tilde{\boldsymbol{\gamma}} \\
& =\tilde{\boldsymbol{\gamma}}^{T}\left(\boldsymbol{R} \hat{\mathbf{V}}_{G} \boldsymbol{R}^{T}\right)^{-1} \tilde{\boldsymbol{\gamma}} \\
& =(\boldsymbol{R} \tilde{\boldsymbol{\beta}}-\boldsymbol{r})^{T}\left(\boldsymbol{R} \hat{\mathbf{V}}_{G} \boldsymbol{R}^{T}\right)^{-1}(\boldsymbol{R} \tilde{\boldsymbol{\beta}}-\boldsymbol{r})
\end{aligned}
$$

onde $\tilde{\boldsymbol{\beta}}$ é obtido minimizando-se a distância

$$
D=\min _{\boldsymbol{\beta}}\left\{(\boldsymbol{R} \hat{\boldsymbol{\beta}}-\boldsymbol{R} \boldsymbol{\beta})^{T}\left(\boldsymbol{R} \hat{\mathbf{V}}_{G} \boldsymbol{R}^{T}\right)^{-1}(\boldsymbol{R} \hat{\boldsymbol{\beta}}-\boldsymbol{R} \boldsymbol{\beta}) \text { sujeito a } \boldsymbol{R} \boldsymbol{\beta} \geq \boldsymbol{r}\right\}
$$

a qual pode ser resolvida através dos algoritmos de programação quadrática (veja, por exemplo, Bazaraa et al., 1993, Capítulo 11 ). Portanto para grandes amostras e $c>0$, a distribuição nula assintótica de $\xi_{k p}$ fica aproximadamente dada por

$$
\begin{aligned}
& P\left(\xi_{k p} \geq c\right)=\sum_{\ell=1}^{k} w(k, \ell ; \hat{\Omega}) P\left(\chi_{\ell}^{2} \geq c\right) \mathrm{e} \\
& P\left(\xi_{k p}=0\right)=w(k, 0 ; \hat{\Omega})
\end{aligned}
$$

onde $\hat{\Omega}=n\left(\boldsymbol{R} \hat{\mathbf{V}}_{G} \boldsymbol{R}^{T}\right)^{-1}$. Se o interesse fosse testar $H_{0}: \boldsymbol{R} \boldsymbol{\beta}=\boldsymbol{r}$ versus $H_{1}^{*}: \boldsymbol{R} \boldsymbol{\beta} \neq \boldsymbol{r}$, a estatística de Wald tomaria a forma

$$
\xi_{w}^{*}=(\boldsymbol{R} \hat{\boldsymbol{\beta}}-\boldsymbol{r})^{T}\left(\boldsymbol{R} \hat{\mathbf{V}}_{G} \boldsymbol{R}^{T}\right)^{-1}(\boldsymbol{R} \hat{\boldsymbol{\beta}}-\boldsymbol{r}),
$$


cuja distribuição nula assintótica é uma qui-quadrado central com $k$ graus de liberdade. É usual comparar as distribuições empíricas de $\xi_{k p}$ e $\xi_{w}^{*}$ no sentido de verificar o ganho de poder com a imposição de restrições na hipótese alternativa. Faremos isso no próximo Capítulo.

\section{Caso II}

O outro caso de interesse seria testar $H_{1}: \boldsymbol{R} \boldsymbol{\beta} \geq \boldsymbol{r}$ versus $H_{2}-H_{1}$, onde $H_{2}: \boldsymbol{\beta} \in \mathbb{R}^{p}$. A estatística de Kodde e Palm toma a seguinte forma nesse caso:

$$
\xi_{k p}=(\boldsymbol{R} \hat{\boldsymbol{\beta}}-\boldsymbol{R} \tilde{\boldsymbol{\beta}})^{T}\left(\boldsymbol{R} \hat{\mathbf{V}}_{G} \boldsymbol{R}^{T}\right)^{-1}(\boldsymbol{R} \hat{\boldsymbol{\beta}}-\boldsymbol{R} \tilde{\boldsymbol{\beta}}) .
$$

Podemos aplicar aqui o lema de Wolak (Lema 3.1) uma vez que a principal condição de regularidade, de que $\sqrt{n}\left(\hat{\boldsymbol{\beta}}_{G}-\boldsymbol{\beta}\right) \stackrel{\mathcal{D}}{\longrightarrow} \mathrm{N}_{p}\left(0, \Sigma^{-1}\right)$, é satisfeita. Portanto, para todo $c>0$ e $\boldsymbol{\beta} \in C^{b}$ temos que

$$
\lim _{n \rightarrow \infty} P\left(\xi_{k p} \geq c \mid \boldsymbol{\beta}\right)=\sum_{\ell=1}^{m} w\left(m, m-\ell ; \boldsymbol{\Omega}_{b}\right) P\left(\chi_{\ell}^{2} \geq c\right)
$$

onde $\boldsymbol{\Omega}_{b}=\boldsymbol{R}_{b} \boldsymbol{\Sigma}^{-1} \boldsymbol{R}_{b}^{T}$ e $\boldsymbol{R}_{b}$ é uma submatriz de dimensão $(m \times k)$ de $\boldsymbol{R}$ consistindo de $m \leq k$ linhas de $\boldsymbol{R}$. A dificuldade, como foi mencionado na Seção 3.4, é encontrar a situação nula menos favorável, isto é, para quais valores de $(m, \boldsymbol{\beta}), 2 \leq m \leq k$, tem-se o menor valor em (3.6.12). Quando a matriz de correlação associada com a matriz $\Omega$ não depende de $\boldsymbol{\beta}$ sob a hipótese nula temos que a situação nula menos favorável é alcançada quando $m=k$. Nesse caso, a distribuição nula menos favorável fica para grandes amostras e $c>0$ dada aproximadamente por

$$
\begin{aligned}
& P\left(\xi_{k p} \geq c\right)=\sum_{\ell=1}^{k} w(k, k-\ell ; \hat{\Omega}) P\left(\chi_{\ell}^{2} \geq c\right) \mathrm{e} \\
& P\left(\xi_{k p}=0\right)=w(k, k ; \hat{\Omega})
\end{aligned}
$$


onde $\hat{\Omega}=n\left(\boldsymbol{R} \hat{\mathrm{V}}_{G} \boldsymbol{R}^{T}\right)^{-1}$. Lembrando que os pesos $w(k, \ell ; \Omega)$ 's são definidos no Apêndice A e dependem apenas da estrutura de correlação (coeficientes de correlação linear associados com a matriz $\Omega$ ).

\subsection{1 g Grupos}

\section{Caso I}

Suponha agora que o interesse seja testar

$$
H_{0}: \mu_{1}=\mu_{2}=\cdots=\mu_{g} \text { versus } H_{1}: \boldsymbol{R} \boldsymbol{\mu} \geq 0
$$

com pelo menos uma desigualdade estrita em $H_{1}, R$ é uma matriz $(k \times g)$ de posto completo. A estatística de Kodde e Palm para testar as hipóteses (3.6.14) fica dada por

$$
\xi_{k p}=(\boldsymbol{R} \tilde{\boldsymbol{\mu}})^{T}\left(\boldsymbol{R} \hat{\mathbf{V}} \boldsymbol{\mu} \boldsymbol{R}^{T}\right)^{-1}(\boldsymbol{R} \tilde{\boldsymbol{\mu}})
$$

cuja distribuição nula assintótica supondo $n_{\ell} \rightarrow \infty, \forall \ell$, é dada por (3.6.13) com $\Omega=$ $\boldsymbol{R} \boldsymbol{\Sigma}_{\boldsymbol{\mu}}^{-1} \boldsymbol{R}^{T}$. Os coeficientes de correlação associados com a matriz $\Omega$ podem ser expressos na forma

$$
\varrho_{i j}=\frac{\sum_{\ell=1}^{g} r_{i \ell} r_{j \ell} \sigma_{\ell}^{2}}{\sqrt{\left(\sum_{\ell=1}^{g} r_{i \ell}^{2} \sigma_{\ell}^{2}\right)\left(\sum_{\ell=1}^{g} r_{j \ell}^{2} \sigma_{\ell}^{2}\right)}}
$$

$i, j=1,2, \ldots, k$, onde $r_{i j}$ denota o $(i, j)$-ésimo elemento de $\boldsymbol{R}$ e $\sigma_{\ell}^{2}$ está sendo avaliado sob $H_{0}$. Note que $\operatorname{Cov}\left(\boldsymbol{y}_{i \ell}\right)=\mathrm{V}_{i \ell}^{1 / 2} \operatorname{Corr}\left(\boldsymbol{y}_{i \ell}\right) \mathrm{V}_{i \ell}^{1 / 2}$, onde $\mathrm{V}_{i \ell}$ é uma matriz diagonal com elementos $V_{i \ell 1}, V_{i \ell 2}, \ldots, V_{i \ell t}$, com $V_{i \ell j}$ sendo a função de variância de $y_{i \ell j}$. Sob $H_{0}$ podemos expressar $\mathrm{V}_{i \ell}=V \mathrm{I}_{t}$, onde $V=V(\mu)$ com $\mu$ sendo o parâmetro comum assumido sob a hipótese nula. Se nós fizermos a suposição de que $\operatorname{Corr}\left(\boldsymbol{y}_{i \ell}\right)$ não depende 
de $\mu$, por exemplo assumindo que $\operatorname{Corr}\left(\boldsymbol{y}_{i \ell}\right)=\delta_{i \ell}(\boldsymbol{\tau})$, onde $\boldsymbol{\tau}$ denota o verdadeiro valor da correlação, então $\sigma_{\ell}^{2}$ dado em (3.5.8) e consequentemente $\varrho_{i j}$ dado em (3.6.16) não dependem de $\mu$. Assim, a distribuição nula assintótica menos favorável de $\xi_{k p}$, dada por (3.6.13), depende somente dos parâmetros de perturbação $(\varrho, \tau)$. Vamos considerar alguns casos particulares.

Inicialmente, vamos supor o caso de ordem simples na hipótese alternativa, em que $H_{1}: \mu_{1} \leq \mu_{2} \leq \cdots \leq \mu_{g}$ com pelo menos uma desigualdade estrita. Aqui, $\boldsymbol{R}$ reduz-se a uma matriz $(g-1) \times g$ cuja $j$-ésima linha tem os valores -1 e 1 nas posições $j$ e $j+1$, respectivamente, e zeros nas demais posições. Os coeficientes de correlação associados com a matriz $\Omega$ ficam dados por

$$
\varrho_{\ell(\ell+1)}=\varrho_{(\ell+1) \ell}=-\left\{\frac{\sigma_{\ell}^{2} \sigma_{\ell+2}^{2}}{\left(\sigma_{\ell+1}^{2}+\sigma_{\ell}^{2}\right)\left(\sigma_{\ell+1}^{2}+\sigma_{\ell+2}^{2}\right)}\right\}^{1 / 2},
$$

$\ell=1,2, \ldots, g-2$ e $\varrho_{\ell \ell}=0$ para $\left|\ell-\ell^{\prime}\right|>1$. A quantidade $\sigma_{\ell}^{2}$ pode ser consistentemente estimada por $n \hat{v}_{\mu_{\ell}}, \ell=1,2, \ldots, g$. A estatística de Kodde e Palm pode ser expressa na forma

$$
\xi_{k p}=\sum_{\ell=1}^{g}\left(\frac{\tilde{\mu}_{\ell}-\hat{\mu}^{0}}{\hat{v}_{\mu_{\ell}}}\right)^{2}
$$

onde $\hat{\mu}^{0}$ é a estimativa comum de $\boldsymbol{\mu}$ sob $H_{0}$. Park et al. (1998) propuseram, recentemente, para o caso de ordem simples em $H_{1}$ a estatística

$$
T_{01}=\sum_{\ell=1}^{g}\left(\frac{\tilde{\mu}_{\ell}-\hat{\mu}^{0}}{\hat{v}_{\mu_{\ell}}^{0}}\right)^{2}
$$

onde $\hat{v}_{\mu_{\ell}}^{0}$ é avaliado em $\hat{\mu}^{0}$ enquanto $\hat{v}_{\mu_{\ell}}$ é avaliado em $\hat{\mu}$. Como $\hat{\boldsymbol{\mu}}^{0}$ e $\hat{\boldsymbol{\mu}}$ são estimativas consistentes de $\boldsymbol{\mu}$, então $\xi_{k p}$ e $T_{01}$ são assintoticamente equivalentes. Quando a estrutura de correlação é dada por (3.6.17) vários estudos têm sido desenvolvidos para o cálculo dos pesos. Em Robertson et al. (1988) são apresentados, por exemplo, os níveis críticos para diversos valores de $\varrho$ e $k$. São também apresentadas aproximações para o caso em que as quantidades $\sigma_{1}^{2}, \sigma_{2}^{2}, \ldots, \sigma_{g}^{2}$ não são muito diferentes. 
Outro caso de interesse prático é o de ordem simples na forma de árvore em que a hipótese alternativa é $H_{1}: \mu_{1} \leq\left[\mu_{2}, \ldots, \mu_{g}\right]$ com pelo menos uma desigualdade estrita. Os coeficientes de correlação associados com a matriz $\Omega$ ficam nesse caso dados por

$$
\varrho_{(\ell-1)\left(\ell^{\prime}-1\right)}=\left\{\frac{\sigma_{\ell}^{2} \sigma_{\ell^{\prime}}^{2}}{\left(\sigma_{1}^{2}+\sigma_{\ell}^{2}\right)\left(\sigma_{1}^{2}+\sigma_{\ell^{\prime}}^{2}\right)}\right\}^{1 / 2},
$$

onde $2 \leq \ell \neq \ell^{\prime} \leq g$. A quantidade $\sigma_{\ell}^{2}$ pode ser consistentemente estimada por $n \hat{v}_{\mu_{\ell}}, \ell=1.2, \ldots, g$ e a estatística de Kodde e Palm fica nesse caso também expressa na forma (3.6.15). Similarmente ao caso de ordem simples na hipótese alternativa, vários estudos têm sido desenvolvidos no sentido de facilitar o cálculo dos pesos $w(k, \ell ; \Omega)$ 's quando a estrutura de correlação associada com a matriz $\Omega$ é dada por (3.6.20) (veja, por exemplo, Robertson et al., 1988).

Nesses dois casos, de ordem simples e ordem simples na forma de árvore em $H_{1}$, a distribuição nula da estatística de Kodde e Palm fica para grandes amostras e $c>0$ aproximadamente dada por

$$
\begin{aligned}
& P\left(\xi_{k p} \geq c\right)=\sum_{\ell=1}^{g-1} w(g-1, \ell ; \hat{\Omega}) P\left(\chi_{\ell}^{2} \geq c\right) \mathrm{e} \\
& P\left(\xi_{k p}=0\right)=w(g-1,0 ; \hat{\Omega}),
\end{aligned}
$$

onde $w(g-1, \ell ; \hat{\Omega})$ depende apenas das correlações (3.6.17) e (3.6.20), respectivamente, avaliadas em $\hat{\boldsymbol{\mu}}$.

\section{Caso II}

Com relação ao caso II vamos considerar a situação de testar $H_{1}: \boldsymbol{R} \boldsymbol{\mu} \geq \mathbf{0}$ versus $H_{2}$ $H_{1}$, onde $H_{2}: \boldsymbol{\mu} \in \mathbb{R}^{g}$ e $\boldsymbol{R}$ é uma matriz $(k \times g)$ de posto completo. Podemos mostrar 
facilmente que se a verdadeira matriz de correlação $\operatorname{Corr}\left(\boldsymbol{y}_{i \ell}\right)$, referente ao $i$-ésimo elemento do $\ell$-ésimo grupo, não depende de $\boldsymbol{\mu}$, por exemplo assumindo que $\operatorname{Corr}\left(\boldsymbol{y}_{i \ell}\right)=$ $\delta_{i \ell}(\tau)$, os coeficientes de correlação associados com a matriz $\Omega_{b}=R_{b} \Sigma_{\mu}^{-1} \boldsymbol{R}_{b}^{T}$, onde $\boldsymbol{R}_{b}$ é uma submatriz de dimensão $(m \times g)$ que contém $m$ linhas de $\boldsymbol{R}$, não dependem de $\boldsymbol{\mu}$ sob $\boldsymbol{R}_{b} \boldsymbol{\mu}=0$ para algumas estruturas de $\boldsymbol{R}$, ou seja, sempre que $\boldsymbol{R} \boldsymbol{\mu}=\mathbf{0}$ implicar em $\mu_{1}=\mu_{2}=\cdots,=\mu_{g}$, como por exemplo ordem simples, em que $H_{1}: \mu_{1} \geq \mu_{2} \geq \cdots \geq \mu_{g}$, e ordem simples na forma de árvore, em que $H_{1}: \mu_{1} \geq\left[\mu_{2}, \ldots, \mu_{g}\right]$. Nesses dois casos a estatística de Kodde e Palm fica expressa na forma

$$
\xi_{k p}=\sum_{\ell=1}^{g}\left(\frac{\hat{\mu}_{\ell}-\tilde{\mu}_{\ell}}{\hat{\sigma}_{\ell}^{2}}\right)^{2}
$$

e tem para grandes amostras e $c>0$ distribuição nula menos favorável dada aproximadamente por

$$
\begin{aligned}
& P\left(\xi_{k p} \geq c\right)=\sum_{\ell=1}^{g-1} w(g-1, g-1-\ell ; \hat{\Omega}) P\left(\chi_{\ell}^{2} \geq c\right) \mathrm{e} \\
& P\left(\xi_{k p}=0\right)=w(g-1, g-1 ; \hat{\Omega})
\end{aligned}
$$

onde $w(g-1, \ell ; \hat{\Omega})$ depende apenas dos coeficientes de correlação (3.6.17) e (3.6.20), respectivamente, avaliados em $\hat{\mu}$.

\subsection{Aplicações}

\subsubsection{Processo infeccioso pulmonar}

Em um estudo de caso-controle realizado no Setor de Anatomia e Patologia do Hospital Heliópolis, em São Paulo-SP, no período de 1970 a 1982 Paula e Tuder (1986), 
175 pacientes com processo infeccioso pulmonar foram classificados de acordo com as seguintes variáveis:

1. Resp: tipo de tumor (1: maligno, 0: benigno);

2. Idade: idade em anos;

3. Sexo (1: masculino, 2: feminino);

4. HL: intensidade da célula histiócitos-linfócitos (1: ausente, 2: discreta, 3: moderada, 4: intensa) e

5. FF: intensidade da célula fibrose-frouxa (1: ausente, 2: discreta, 3: moderada, 4: intensa);

As informações referentes às variáveis Resp, HL e FF foram obtidas após biópsia realizada na região pleural de cada paciente ou por autópsia no caso de óbito. Os dados são apresentados no Apêndice D.

Considere, como exemplo, a aplicação do modelo logístico linear apenas com os efeitos principais

$$
P(\operatorname{Resp}=1 \mid \eta)=\{1+\exp (-\eta)\}^{-1}
$$

onde

$$
\eta=\beta_{1}+\beta_{2} \text { Idade }+\beta_{3} \text { Sexo }+\sum_{i=1}^{4} \beta_{4 i} \mathrm{HL}_{i}+\sum_{i=1}^{4} \beta_{5 i} \mathrm{FF}_{i},
$$

com $\mathrm{HL}_{i}$ e $\mathrm{FF}_{i}$ sendo variáveis binárias correspondentes aos níveis de $\mathrm{HL}$ e FF, respectivamente. É assumido que $\beta_{41}=\beta_{51}=0$. Uma observação importante é que devido ao fato da amostragem ter sido retrospectiva, o uso do modelo acima para fazer previsões somente é válido corrigindo-se a estimativa da constante, $\beta_{1}$ (veja, por exemplo, McCullagh e Nelder, 1989, p. 113). 
Do ponto de vista médico é muito razoável supor que a chance de tumor maligno no nível $i$ de HL ou FF não é maior que no nível $i+1$. Assim, pode ser de interesse testar as seguintes hipóteses:

$$
\begin{array}{lll}
H_{0}: \beta_{42}=\beta_{43}=\beta_{44}=0 \text { versus } & H_{1}: \beta_{44} \leq \beta_{43} \leq \beta_{42} \leq 0 \mathrm{e} \\
H_{0}: \beta_{52}=\beta_{53}=\beta_{54}=0 & \text { versus } & H_{1}: \beta_{54} \leq \beta_{53} \leq \beta_{52} \leq 0
\end{array}
$$

com pelo menos uma desigualdade estrita em $H_{1}$. Ou seja, verificar se há pelo menos dois grupos de intensidade em cada caso com chances diferentes de tumor maligno. Para testar as hipóteses acima separadamente a matriz $\boldsymbol{R}$ toma, respectivamente, a seguinte forma:

$$
\boldsymbol{R}=\left(\begin{array}{rrrrrrrrr}
0 & 0 & 0 & 1 & -1 & 0 & 0 & 0 & 0 \\
0 & 0 & 0 & 0 & 1 & -1 & 0 & 0 & 0 \\
0 & 0 & 0 & -1 & 0 & 0 & 0 & 0 & 0
\end{array}\right)
$$

e

$$
\boldsymbol{R}=\left(\begin{array}{rrrrrrrrr}
0 & 0 & 0 & 0 & 0 & 0 & 1 & -1 & 0 \\
0 & 0 & 0 & 0 & 0 & 0 & 0 & 1 & -1 \\
0 & 0 & 0 & 0 & 0 & 0 & -1 & 0 & 0
\end{array}\right)
$$

A Tabela 3.7.3 apresenta as estimativas de máxima verossimilhança (desvio padrão assintótico entre parênteses) dos parâmetros do modelo (3.7.1). Nota-se, pelas estimativas não restritas, que ocorre uma única violação entre as estimativas correspondentes aos níveis $\mathrm{FF}(3)$ e $\mathrm{FF}(4)$. A violação foi corrigida calculando-se as estimativas restrita de acordo com o procedimento descrito na Subseção 3.2.6. As estimativas restritas (desvio padrão assintótico entre parênteses) também estão na Tabela 3.7.3. O desvio correspondente ao ajuste do modelo restrito foi de $D(\boldsymbol{y} ; \boldsymbol{\mu})=158,98(166 \mathrm{gl})$, indicando um ajuste adequado.

De acordo com as estimativas dos parâmetros nota-se que a chance de tumor maligno (com relação a benigno) cresce com o aumento da idade e que as mulheres são mais 
Tabela 3.7.3: Estimativas não restritas e restritas com o respectivo desvio padrão assintótico dos parâmetros do modelo logístico linear.

\begin{tabular}{lcc}
\hline & \multicolumn{2}{c}{ Estimativa (desvio padrão) } \\
\cline { 2 - 3 } Efeito & Não restrita & Restrita \\
\hline Constante & $-1,850(1,060)$ & $-1,846(1,056)$ \\
Idade & $0,065(0,013)$ & $0,065(0,013)$ \\
Sexo & $-0,784(0,469)$ & $-0,778(0,467)$ \\
HL(2) & $-0,869(0,945)$ & $-0,891(0,944)$ \\
HL(3) & $-2,249(0.968)$ & $-2,210(0,967)$ \\
HL(4) & $-3,295(1,466)$ & $-3,344(1,471)$ \\
FF(2) & $-0,687(0,502)$ & $-0,690(0,500)$ \\
FF(3) & $-1,025(0,525)$ & $-0,874(0,505)$ \\
FF(4) & $0,431(1,123)$ & $-0,874(0,505)$ \\
\hline
\end{tabular}

susceptíveis a desenvolverem processo infeccioso pulmonar que os homens. Paula (1995) verifica através de métodos de diagnóstico que dois pacientes com um perfil atípico são altamente influentes na violação observada entre as estimativas dos níveis $\mathrm{FF}(3)$ e FF(4). Para os níveis do fator HL a estatística da razão de verossimilhança (3.3.10) para testar $H_{0}$ versus $H_{1}$, forneceu o valor $\xi_{r v}=15,67$ e para os níveis do fator $\mathrm{FF}$ o valor da mesma foi de $\xi_{r v}=3,57$. De (3.3.9) com $\hat{\Omega}=\boldsymbol{R}\left(\boldsymbol{X}^{T} \hat{\mathrm{V}} \boldsymbol{X}\right)^{-1} \boldsymbol{R}^{T}$ e os pesos calculados como no Apêndice A para $k=3$ chega-se, respectivamente, aos seguintes 
níveis descritivos:

$$
\begin{aligned}
P & =P\left(\xi_{r v} \geq 15,67\right)=\sum_{\ell=1}^{3} w(3, \ell ; \hat{\Omega}) P\left(\chi_{\ell}^{2} \geq 15,67\right) \\
& =0,377 \times P\left(\chi_{1}^{2} \geq 15,67\right)+0,375 \times P\left(\chi_{2}^{2} \geq 15,67\right)+0,123 \times P\left(\chi_{3}^{2} \geq 15,67\right) \\
& =0,0003 \mathrm{e} \\
P & =P\left(\xi_{r v} \geq 3,57\right)=\sum_{\ell=1}^{3} w(3, \ell ; \hat{\Omega}) P\left(\chi_{\ell}^{2} \geq 3,57\right) \\
& =0,438 \times P\left(\chi_{1}^{2} \geq 3,57\right)+0,295 \times P\left(\chi_{2}^{2} \geq 3,57\right)+0,062 \times P\left(\chi_{3}^{2} \geq 15,67\right) \\
& =0,0946 .
\end{aligned}
$$

Portanto, pode-se afirmar que há pelo menos dois tipos de intensidade para a célula HL com chances diferentes de tumor maligno. Está implícito que essas chances estão em ordem não decrescente. Por outro lado, para o tipo de célula FF, não há evidências fortes de diferença entre as chances de tumor maligno segundo o grau de intensidade.

\subsubsection{Pacientes com ataque epiléptico}

Diggle et al. (1994, Seção 8.4) discutem um exemplo com dados correlacionados e distribuição não normal de um experimento clínico com 59 pacientes epilépticos, os quais foram tratados aleatoriamente ou com a droga anti-epiléptica progabide ou com o placebo. Os dados são apresentados no Apêndice D. Para cada paciente foram contados o número de ataques epilépticos durante as 8 semanas imediatamente anteriores ao tratamente. Após o tratamento, a cada intervalo de 2 semanas, foram contados o número ataque epilépticos durante as 8 semanas imediatamente seguintes ao tratamento. Um modelo de Poisson com parâmetros não restritos foi considerado para ajustar os dados. Entretanto, é razoável esperar que a razão entre a droga e o placebo após o tratamento não seja maior que a razão antes do tratamento. É interessante, então, saber se a droga progabide reduz ou não a razão de ataques epilépticos. 
Seja $y_{i j k}$ o número de ataques epilépticos sofridos pelo $k$-ésimo paciente do $i$-ésimo grupo na $j$-ésima ocasião. É assumido que $y_{i j k} \sim P\left(\lambda_{i j} t_{j}\right)$, onde $t_{j}$ denota o número de semanas do $j$-ésimo período, para $i=1,2 ; j=0,1,2,3,4$ e $k=1,2, \ldots, n_{i j}$ com $n_{1 j}=28$ (grupo placebo), $n_{2 j}=31$ (grupo tratado) e $t_{0}=8, t_{1}=t_{2}=t_{3}=t_{4}=2$. Além disso, será assumida uma matriz de correlação de trabalho simétrica $\Gamma(\rho)$ para o $k$-ésimo paciente do $i$-ésimo grupo, onde $\rho$ é o coeficiente de correlação. O seguinte modelo log-linear foi adotado para explicar a razão de ataques:

$$
\begin{aligned}
& \log \left(\lambda_{01}\right)=\alpha, \\
& \log \left(\lambda_{1 j}\right)=\alpha+\beta, \\
& \log \left(\lambda_{20}\right)=\alpha+\gamma \mathrm{e} \\
& \log \left(\lambda_{2 j}\right)=\alpha+\gamma+\beta+\delta,
\end{aligned}
$$

para $j=1,2,3,4$, onde $\alpha$ é o instante inicial do tratamento, $\beta$ é o efeito do tratamento, $\gamma$ é o efeito do grupo e $\delta$ é a interação entre tratamento e grupo. Note que $\delta$ representa a diferença no logaritmo da razão entre os grupos tratado e placebo após o tratamento e antes do tratamento, isto é,

$$
\delta=\log \left\{\frac{\lambda_{2 j}}{\lambda_{1 j}}\right\}-\log \left\{\frac{\lambda_{20}}{\lambda_{10}}\right\} .
$$

Com relação à média $\mu_{i j}$ temos o modelo

$$
\log \left(\mu_{i j}\right)=\log \left(t_{j}\right)+\log \left(\lambda_{i j}\right)
$$

onde $\log \left(t_{j}\right)$ é um offset. Espera-se que a razão de ataques epilépticos entre os grupos tratados e placebos não cresça após o tratamento, isso é equivalente a impor a restrição $\delta \leq 0$. Para detectar se a droga progabide reduz a razão de ataques epilépticos devemos testar as hipóteses $H_{0}: \delta=0$ versus $H_{1}: \delta<0$. Sob $H_{0}$, a estatística de Wald (3.6.10) tem distribuição $\chi_{1}^{2}$ enquanto a estatística de Kodde e Palm (3.6.8) segue uma distribuição $\frac{1}{2}+\frac{1}{2} \chi_{1}^{2}$. Como $\hat{\delta}=-0,105(0,213)<0$, então $\tilde{\delta}=\hat{\delta}$ e essas estatísticas 
tomam o mesmo valor, $\xi_{w}=\xi_{k p}=(-0,105 / 0,213)^{2}=0,243$, com níveis descritivos dados por $P=0,622$ e $P=0,311$, respectivamente, indicando que o efeito da droga progabide não é significante. Entretanto, se o paciente \#207 (vide Apêndice D, Tabela D.4.1) cujo número de ataques epilépticos antes e depois do tratamento é não usual, for excluído da análise, essas estatísticas tomam o valor $\xi_{w}=\xi_{k p}=(-0,302 / 0,171)^{2}=$ 3,119 , com níveis descritivos dados, respectivamente, por 0,077 e 0,039 . Então podemos concluir baseado na estatística $\xi_{k p}$ ao nível de $5 \%$ de significância que a droga progabide reduz a razão de ataques epilépticos. Ao contrário, com base na estatística $\xi_{w}$ não podemos detectar uma diferença significativa, ao nível de 5\%, no tratamento com a droga progabide.

\subsubsection{Suplemento para redução de pólipos}

Em um estudo realizado para verificar a influência da fibra de trigo e das vitaminas $\mathrm{C}$ e E na redução de pólipos retais (Park et al., 1998), 58 pacientes foram aleatorizados de modo que 22 pacientes receberam um placebo, 16 pacientes receberam um suplemento de vitaminas $\mathrm{C}$ e $\mathrm{E}$ e 20 pacientes receberam um suplemento de vitaminas $\mathrm{C}$ e $\mathrm{E}$ mais fibra de trigo. Após receberem o tratamento os pacientes foram examinados a cada trimestre durante 4 anos. Em cada visita médica foram contados o número de pólipos. Em geral os pólipos não foram removidos completamente, porém o número de pólipos diminuiu frequentemente. O principal objetivo do estudo é testar se há diferença significativa entre os diferentes tratamentos na redução de pólipos retais em uma população de alto risco. Stukel (1993) aplicou o método de EEGs de Liang e Zeger, com resposta marginal Poisson, mas não detectou diferença significativa entre os tratamentos ao nível de $10 \%$ de significância. 
Seja $\mu_{\ell}$ a média do grupo $\ell, \ell=1,2,3$. O interesse é testar $H_{0}: \mu_{1}=\mu_{2}=\mu_{3}$ versus $H_{1}: \mu_{1} \geq \mu_{2} \geq \mu_{3}$ com pelo menos uma desigualdade estrita. Para as duas estruturas de correlação, AR(1) e simétrica, as estimativas EEGs não restritas das médias dos grupos foram calculadas e satisfazem a restrição de ordem $\hat{\mu}_{1} \geq \hat{\mu}_{2} \geq \hat{\mu}_{3}$, portanto, as estimativas restritas são as mesmas, isto é, $\tilde{\mu}_{\ell}=\hat{\mu}_{\ell}, \ell=1,2,3$. Essas estimativas juntamente com a estimativa comum da média sob $H_{0}$ e os respectivos desvios padrão são apresentados na Tabela 3.7.4.

Tabela 3.7.4: Estimativas e estatísticas do teste para os dados do tratamento de pólipos com vitaminas $\mathrm{C}$ e $\mathrm{E}$ mais fibra de trigo.

\begin{tabular}{|c|c|c|c|c|c|}
\hline & \multicolumn{3}{|c|}{$\begin{array}{c}\text { Estimativa EEGs } \\
\text { (Desvio padrão) }\end{array}$} & \multicolumn{2}{|c|}{$\begin{array}{c}\text { Estatísticas do teste } \\
\text { ( } P \text {-valor })\end{array}$} \\
\hline & $\tilde{\mu}_{1}$ & $\tilde{\mu}_{2}$ & $\tilde{\mu}_{3}$ & $\xi_{k p}$ & $T_{01}$ \\
\hline \multirow[t]{2}{*}{ Correlação AR(1) } & 8,0462 & 6,2845 & 5,1699 & 2,9769 & 3,0500 \\
\hline & $(1,2635)$ & $(1,3185)$ & $(1,2770)$ & $(0,0561)$ & $(0,0767)$ \\
\hline \multicolumn{6}{|c|}{$\hat{\mu}^{0}=6,5604(1,1603)$} \\
\hline \multirow[t]{3}{*}{ Correlação simétrica } & 8,4608 & 6,5393 & 4,8108 & 3,3018 & 2,4300 \\
\hline & $(1,2578)$ & $(1,3439)$ & $(1,2692)$ & $(0,0472)$ & $(0,1091)$ \\
\hline & \multicolumn{3}{|c|}{$\hat{\mu}^{0}=6,6695(1,1637)$} & & \\
\hline
\end{tabular}

As duas estruturas de correlação apresentaram resultados similares. Como os dados foram coletados por um longo período de tempo, o modelo com estrutura de correlação $A R(1)$ pode ser mais adequado para explicar a correlação entre as respostas da mesma unidade experimental (vide Park et al., 1998). Ao nível de $10 \%$ de significância, a estatística $T_{01}$ proposta por Park et al. (1998) e dada em (3.6.19), detecta uma diferença significativa entre as médias dos grupos com $P$-valor $=0,0767$, no entanto, a estatística 
de Kodde e Palm dada em (3.6.18), detecta essa diferença com $P$-valor $=0,0561$, indicando com mais evidência que existe uma tendência a diminuir a contagem de pólipos com a combinação de fibra de trigo com as vitaminas $\mathrm{C}$ e E. 


\section{Capítulo 4}

\section{Estudos de Simulação}

\subsection{Introdução}

Nestes estudos vamos assumir que $\boldsymbol{y}_{i}=\left(y_{i 1}, y_{i 2}, \ldots, y_{i t}\right)^{T}$ é um vetor $(t \times 1)$ de respostas e $\boldsymbol{X}_{i}=\left(\boldsymbol{x}_{i 1}, \boldsymbol{x}_{i 2}, \ldots, \boldsymbol{x}_{i t}\right)^{T}$ é uma matriz $(t \times p)$ de valores das covariáveis para a $i$-ésima unidade experimental, $i=1,2, \ldots, n$. Assumimos também que a densidade marginal de $y_{i j}$ pertence à família exponencial, sendo dada por

$$
f\left(y_{i j} ; \theta_{i j}, \phi\right)=\exp \left\{\phi\left[y_{i j} \theta_{i j}-b\left(\theta_{i j}\right)\right]+c\left(y_{i j}, \phi\right)\right\}
$$

onde $\mathrm{E}\left(y_{i j}\right)=\mu_{i j}=b^{\prime}\left(\theta_{i j}\right), \operatorname{Var}\left(y_{i j}\right)=\phi^{-1} b^{\prime \prime}\left(\theta_{i j}\right)$ e $g\left(\mu_{i j}\right)=\eta_{i j}=\boldsymbol{x}_{i j}^{T} \boldsymbol{\beta}$.

Foram consideradas amostras de tamanhos $n=20,50$ e 100 com $t=3$ e para cada observação gerada, $\boldsymbol{y}_{i}=\left(y_{i 1}, y_{i 2}, y_{i 3}\right)^{T}$, é assumida uma estrutura de correlação simétrica, $\operatorname{Corr}\left(y_{i j}, y_{i k}\right)=\rho, \forall j \neq k$, ou $\operatorname{AR}(1), \operatorname{Corr}\left(y_{i j}, y_{i k}\right)=\rho^{|j-k|}, \forall j, k$. Foram geradas amostras de observações correlacionadas das distribuições binomial, Poisson e gama. Para gerar variáveis com distribuição binomial correlacionadas, foi usado o procedi- 
mento descrito por Park et al. (1996) e para gerar variáveis com distribuição de Poisson e gama correlacionadas foi usado o procedimento descrito em Park e Shin (1998). Esses procedimentos e os algoritmos de geração são apresentados no Apêndice B.

A estatística de Kodde e Palm, $\xi_{k p}$, para testar as hipóteses

$$
H_{0}: \boldsymbol{R} \boldsymbol{\beta}=\mathbf{0} \text { versus } H_{1}: \boldsymbol{R} \boldsymbol{\beta} \geq \mathbf{0},
$$

com pelo menos uma desigualdade estrita em $H_{1}$, foi comparada com a estatística de Wald, $\xi_{w}$, para hipótese alternativa não restrita, para verificar o ganho de poder. $O$ poder foi estimado como sendo a proporção de valores de $\xi_{k p}$ e $\xi_{w}$ maiores que $c_{\alpha}$, o valor crítico assintótico correspondente ao nível de significância $\alpha$. Para a estatística $\xi_{w}$ o valor crítico é tal que $P\left(\chi_{2}^{2} \geq c_{\alpha}\right)=\alpha$ e para a estatística $\xi_{k p}, c_{\alpha}$ é tal que

$$
\alpha=w\left(2,0 ; \Omega^{*}\right)+w\left(2,1 ; \Omega^{*}\right) P\left(\chi_{1}^{2} \geq c_{\alpha}\right)+w\left(2,2 ; \Omega^{*}\right) P\left(\chi_{2}^{2} \geq c_{\alpha}\right)
$$

onde $w\left(2, \ell ; \Omega^{*}\right), \ell=0,1,2$, são dados no Apêndice $\mathrm{A}, \boldsymbol{\Omega}^{*}=\boldsymbol{R} \mathrm{V}_{G} \boldsymbol{R}^{T}$ com $\mathrm{V}_{G}$ sendo avaliado no verdadeiro valor de $\boldsymbol{\beta}$ e $\chi_{\ell}^{2}$ denota uma variável aleatória com distribuição qui-quadrado central com $\ell$ graus de liberdade. A estimativa não restrita do vetor de parâmetros $\boldsymbol{\beta}$ foi obtida através da macro GEE versão 2.02 do SAS (GEE: A SAS macro for Longitudinal Data Analysis) elaborada por Ulrike Gröemping, Universitaet Dortmun disponivel na internet. ${ }^{1}$ A estimativa restrita foi obtida usando-se a subrotina LCP do SAS/IML (SAS Intitute Inc., 1988). Cada procedimento foi repetido 1000 vezes. Toda parte computacional foi realizada em um microcomputador Pentium II 400 e os programas elaborados para serem processados no PROC IML do SAS Release 6.12.

\footnotetext{
${ }^{1}$ http://www.statlab.uni-heidelberg.de/statlib/GEE/GEE1/
} 


\subsection{Distribuição binomial}

Foram geradas amostras independentes de $\boldsymbol{y}_{\boldsymbol{i}}=\left(y_{i 1}, y_{i 2}, y_{i 3}\right)^{T}$ com $y_{i j} \sim B\left(1, \mu_{j}\right)$, $j=1,2,3, i=1,2, \ldots, n$ e com estruturas de correlação simétrica e $\operatorname{AR}(1)$ para as observações da mesma unidade experimental. As simulações foram conduzidas para verificar o poder da estatística de Kodde Palm para testar as seguintes hipóteses:

$$
H_{0}: \mu_{1}=\mu_{2}=\mu_{3} \text { versus } H_{1}: \mu_{1} \geq \mu_{2} \geq \mu_{3}
$$

com pelo menos uma desigualdade estrita em $H_{1}$, para valores de $\rho=0,0 ; 0,1 ; 0,3$ e 0,7 , supondo em $H_{0}$ que $\mu_{1}=\mu_{2}=\mu_{3}=1 / 2$ e na alternativa $\mu_{1}=\mu_{2}=1 / 2$ e $\mu_{3}=1 / 2-\delta$, para $\delta=0,00 ; 0,10 ; 0,20$; e 0,30 . Quando $\rho=0,7$ e $\delta=0,20$ e 0,30 , a condição (2.4) de Park et al. (1996) não é satisfeita e portanto não podemos gerar amostras de variáveis binárias correlacionadas.

Usando o modelo logístico linear, $\beta_{j}=\operatorname{logit}\left(\mu_{j}\right)=\ln \left\{\mu_{j} /\left(1-\mu_{j}\right)\right\}, j=1,2,3$, as hipóteses acima são equivalentes às hipóteses (4.1.1), onde

$$
\boldsymbol{\beta}=\left(\begin{array}{c}
\beta_{1} \\
\beta_{2} \\
\beta_{3}
\end{array}\right) \text { e } \boldsymbol{R}=\left[\begin{array}{ccc}
1 & -1 & 0 \\
0 & 1 & -1
\end{array}\right]
$$

Dos resultados obtidos podemos observar que, para a estrutura de correlação simétrica, quando $n=20$ e $\rho=0$, não houve diferença significativa entre as distribuições empírica e teórica para a estatística de Kodde e Palm. Essa situação corresponde ao caso de independência de todas as observações. Essas distribuições são significativamente diferentes para $n=20$ e $\rho>0$. A partir de $n=50$, não houve diferença significativa entre as distribuições empírica e teórica para a estatística $\xi_{k p}$, tanto para observações fracamente correlacionadas como para observações fortemente correlacionadas. Com a 
estrutura de correlação $\mathrm{AR}(1)$, o comportamento dessas distribuições é semelhante para todos os tamanhos de amostras, sendo as mesmas significativamente diferentes para observações altamente correlacionadas, $\rho \geq 0,3$.

Isso é confirmado pela aplicação do teste de Kolmogorov-Smirnov cujos resultados são apresentados na Tabela 4.2.1 (P-valor entre parênteses).

Tabela 4.2.1: Binomial: Kolmogorov-Smirnov (P-valor).

\begin{tabular}{cccc}
\hline \multicolumn{4}{c}{ Tamanho da amostra } \\
\hline$\rho$ & $n=20$ & $n=50$ & $n=100$ \\
\hline \multicolumn{4}{c}{ Correlação simétrica } \\
\hline \hline 0,0 & $0,0362(0,3790)$ & $0,0294(0,6455)$ & $0,0132(0,9999)$ \\
0,1 & $0,0598(0,0218)$ & $0,0223(0,9118)$ & $0,0252(0,8170)$ \\
0,3 & $0,0571(0,0325)$ & $0,0206(0,9513)$ & $0,0254(0,8095)$ \\
0,7 & $0,1234(0,0000)$ & $0,0299(0,6244)$ & $0,0181(0,9858)$ \\
\hline & & Correlação AR(1) \\
\hline \hline 0,0 & $0,0214(0,9343)$ & $0,0176(0,9894)$ & $0,0242(0,8537)$ \\
0,1 & $0,0197(0,9669)$ & $0,0270(0,7477)$ & $0,0343(0,4480)$ \\
0,3 & $0,0721(0,0028)$ & $0,0595(0,0228)$ & $0,0675(0,0063)$ \\
0,7 & $0,1576(0,0000)$ & $0,1215(0,0000)$ & $0,1275(0,0000)$ \\
\hline
\end{tabular}

Para $\rho=0 ; 0,1 ; 0,3$ e 0,7, as Figuras 4.2.1, 4.2 .2 e 4.2 .3 a seguir mostram, para a estatística $\xi_{k p}$, a distribuição empírica e a distribuição teórica dada por

$$
P\left(\xi_{k p} \geq c\right)=\sum_{\ell=0}^{2} w(2, \ell ; \hat{\Omega}) P\left(\chi_{\ell}^{2} \geq c\right),
$$

para a estrutura de correlação simétrica entre as observações da mesma unidade experimental e amostras de tamanho 20, 50 e 100, respectivamente. 
Figura 4.2.1: Binomial $n=20$ : distribuições empírica e teórica da estatística $\xi_{k p}$ com estrutura de correlação simétrica.
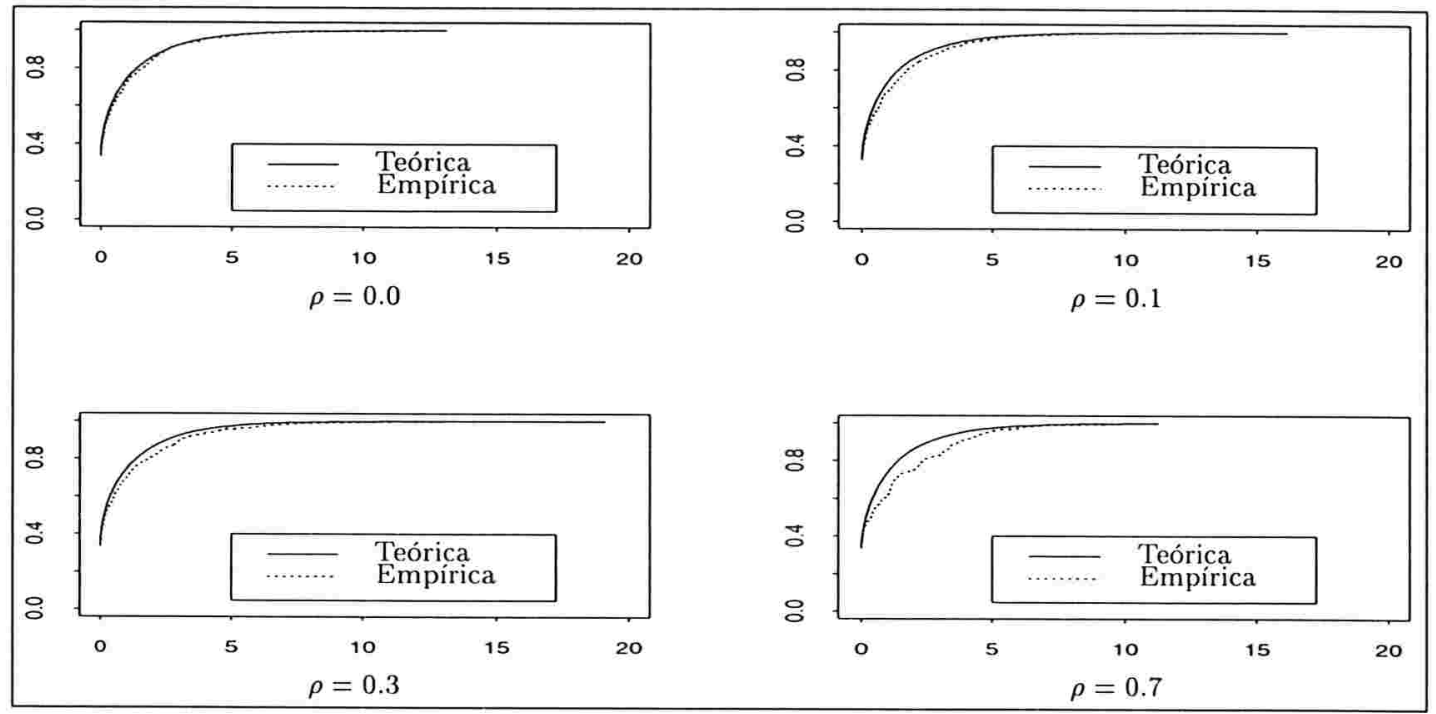

Figura 4.2.2: Binomial $n=50$ : distribuições empírica e teórica da estatística $\xi_{k p}$ com estrutura de correlação simétrica.
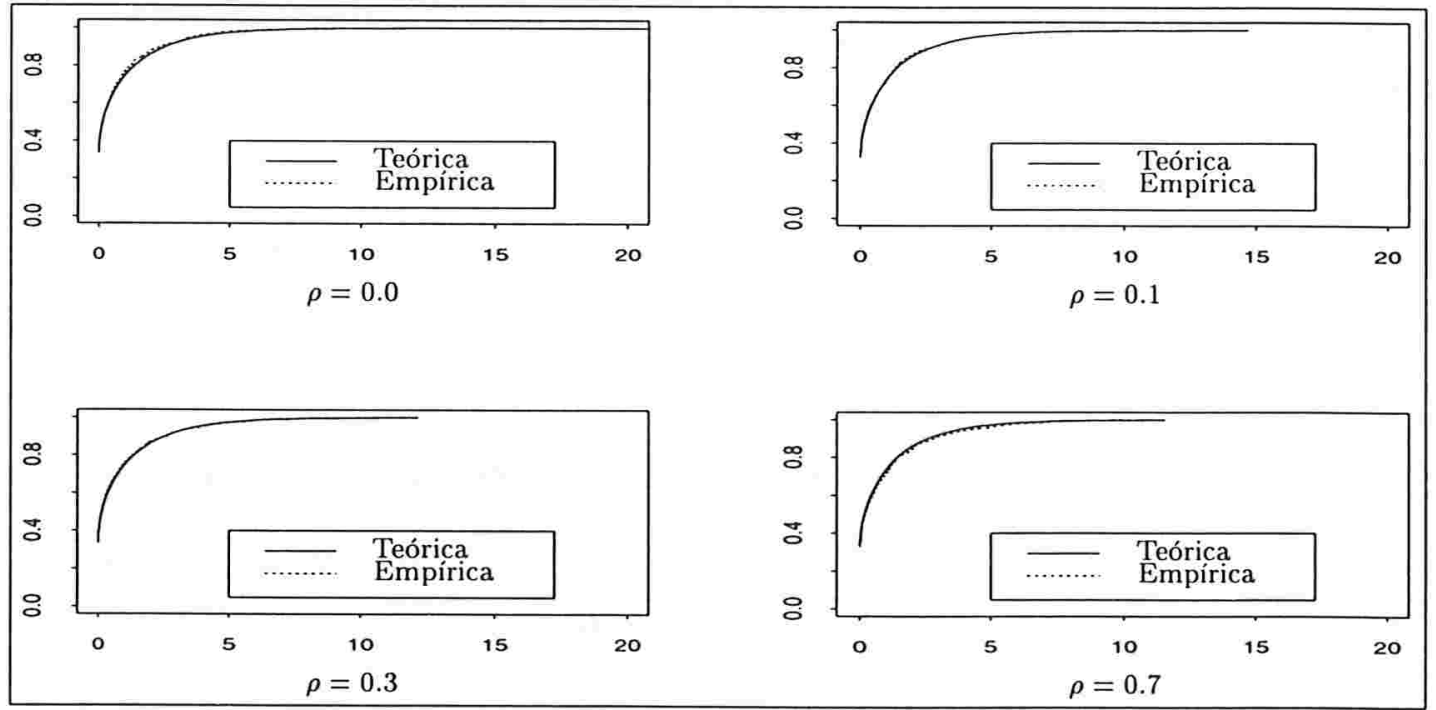
Figura 4.2.3: Binomial $n=100$ : distribuições empírica e teórica da estatística $\xi_{k p}$ com estrutura de correlação simétrica.

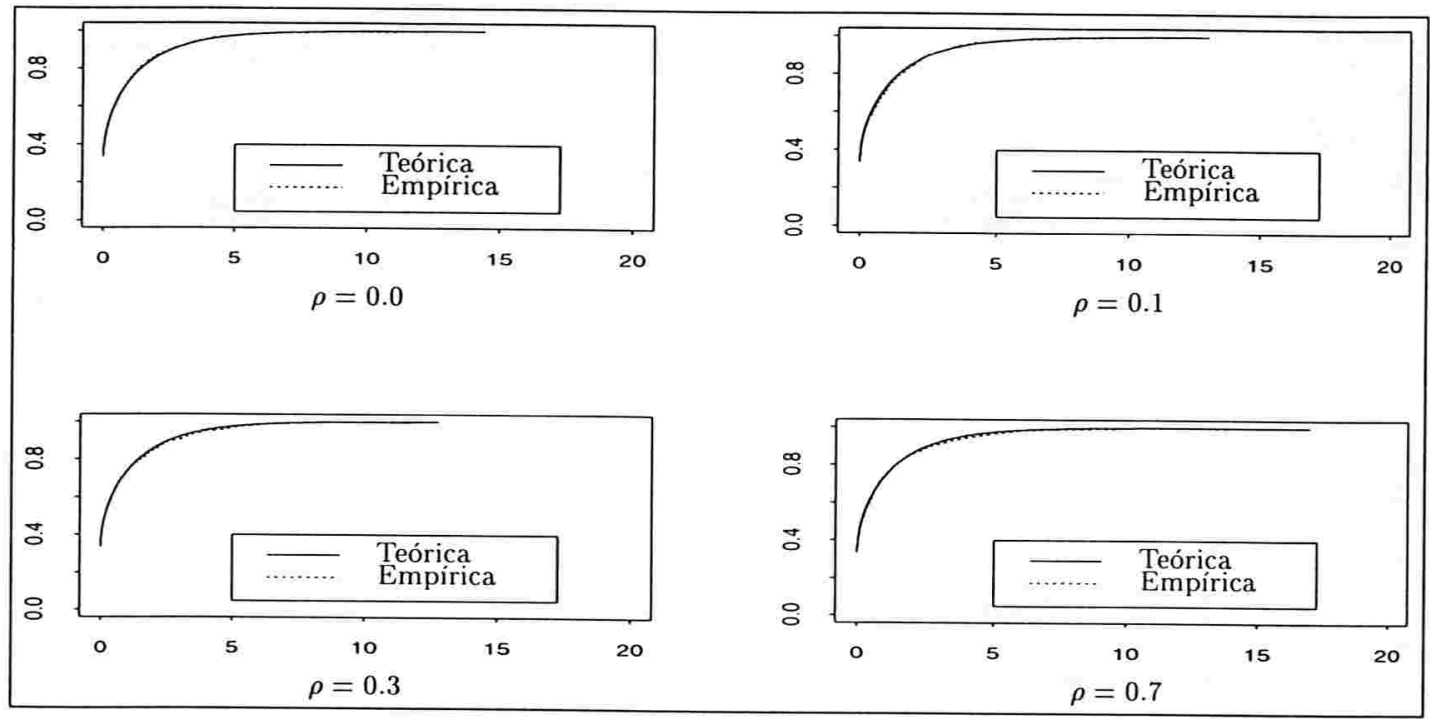

As Tabelas 4.2.2, 4.2.3 e 4.2.4, apresentam o poder estimado dos testes $\xi_{k p}$ e $\xi_{w}$ para amostras de tamanhos 20,50 e 100, respectivamente, com réplicas de $t=3$ e estruturas de correlação simétrica e $\operatorname{AR}(1)$. O poder da estatística de Kodde e Palm, $\xi_{k p}$, é sempre superior ao poder da estatística de Wald, $\xi_{w}$, como esperado, uma vez que $\xi_{k p}$ leva em consideração a restrição imposta sobre o vetor de parâmetros $\boldsymbol{\beta}$. Os tamanhos empíricos de ambos os testes superestimam em geral o valor nominal e ficam mais próximos desse último para correlações baixas e à medida que aumenta o tamanho da amostra. $\mathrm{O}$ poder dos testes cresce à medida que aumenta a correlação entre as observações. Esse resultado, em princípio, é contrário ao resultado apresentado por Park et al. (1998) para o mesmo tipo de problema, porém supondo correlações dentro de cada grupo. Park et al. (1998) verificam que o poder decresce com o aumento da correlação. No nosso estudo, entretanto, as correlações são no tempo e o que se compara são as médias no tempo. Como mostramos no Apêndice $\mathrm{C}$ para o caso de $t=2$ é esperado um 
aumento de poder com a redução da correlação quando as correlações ocorrem dentro dos grupos e o contrário quando as correlações ocorrem no tempo.

Tabela 4.2.2: Binomial: poder das estatísticas $\xi_{k p}$ e $\xi_{w}$.

\begin{tabular}{|c|c|c|c|c|c|c|c|}
\hline \multicolumn{2}{|c|}{$n=20$} & \multicolumn{2}{|c|}{$\alpha=0,01$} & \multicolumn{2}{|c|}{$\alpha=0,05$} & \multicolumn{2}{|c|}{$\alpha=0,10$} \\
\hline$\rho$ & $\delta$ & $\xi_{k p}$ & $\xi_{w}$ & $\xi_{k p}$ & $\xi_{w}$ & $\xi_{k p}$ & $\xi_{w}$ \\
\hline \multicolumn{8}{|c|}{ Correlação simétrica } \\
\hline \multirow[t]{4}{*}{0,0} & 0,00 & 0,012 & 0,019 & 0,056 & 0,061 & 0,102 & 0,113 \\
\hline & 0,10 & 0,047 & 0,029 & 0,171 & 0,103 & 0,274 & 0,187 \\
\hline & 0,20 & 0,151 & 0,091 & 0,380 & 0,240 & 0,535 & 0,360 \\
\hline & 0,30 & 0,367 & 0,219 & 0,687 & 0,502 & 0,811 & 0,666 \\
\hline \multirow[t]{4}{*}{0,1} & 0,00 & 0,016 & 0,014 & 0,070 & 0,059 & 0,129 & 0,123 \\
\hline & 0,10 & 0,056 & 0,027 & 0,193 & 0,117 & 0,305 & 0,201 \\
\hline & 0,20 & 0,180 & 0,095 & 0,419 & 0,284 & 0,566 & 0,385 \\
\hline & 0,30 & 0,406 & 0,239 & 0,756 & 0,553 & 0,863 & 0,728 \\
\hline \multirow[t]{4}{*}{0,3} & 0,00 & 0,022 & 0,026 & 0,073 & 0,086 & 0,141 & 0,147 \\
\hline & 0,10 & 0,073 & 0,043 & 0,250 & 0,150 & 0,361 & 0,237 \\
\hline & 0,20 & 0,238 & 0,133 & 0,542 & 0,370 & 0,679 & 0,509 \\
\hline & 0,30 & 0,578 & 0,376 & 0,868 & 0,722 & 0,930 & 0,848 \\
\hline \multirow[t]{4}{*}{0,7} & 0,00 & 0,013 & 0,018 & 0,098 & 0,066 & 0,185 & 0,172 \\
\hline & 0,10 & 0,062 & 0,011 & 0,299 & 0,171 & 0,510 & 0,301 \\
\hline & 0,20 & $* * *$ & $* * *$ & $* * *$ & $* * *$ & $* * *$ & $* * *$ \\
\hline & 0,30 & *** & $* * *$ & $* * *$ & *** & *** & *** \\
\hline \multicolumn{8}{|c|}{ Correlação AR(1) } \\
\hline \multirow[t]{4}{*}{0,0} & 0,00 & 0,017 & 0,008 & 0,058 & 0,049 & 0,100 & 0,099 \\
\hline & 0,10 & 0,043 & 0,021 & 0,169 & 0,091 & 0,251 & 0,164 \\
\hline & 0,20 & 0,168 & 0,064 & 0,392 & 0,255 & 0,510 & 0,375 \\
\hline & 0,30 & 0,350 & 0,211 & 0,669 & 0,492 & 0,806 & 0,637 \\
\hline \multirow[t]{4}{*}{0,1} & 0,00 & 0,011 & 0,012 & 0,059 & 0,061 & 0,107 & 0,106 \\
\hline & 0,10 & 0,037 & 0,023 & 0,174 & 0,089 & 0,269 & 0,171 \\
\hline & 0,20 & 0,154 & 0,079 & 0,402 & 0,243 & 0,522 & 0,374 \\
\hline & 0,30 & 0,389 & 0,226 & 0,697 & 0,515 & 0,811 & 0,656 \\
\hline \multirow[t]{4}{*}{0,3} & 0,00 & 0,019 & 0,015 & 0,067 & 0,068 & 0,117 & 0,128 \\
\hline & 0,10 & 0,055 & 0,032 & 0,179 & 0,106 & 0,303 & 0,177 \\
\hline & 0,20 & 0,189 & 0,104 & 0,476 & 0,301 & 0,605 & 0,429 \\
\hline & 0,30 & 0,487 & 0,291 & 0,780 & 0,612 & 0,876 & 0,750 \\
\hline \multirow[t]{4}{*}{0,7} & 0,00 & 0,010 & 0,004 & 0,072 & 0,049 & 0,162 & 0,118 \\
\hline & 0,10 & 0,076 & 0,018 & 0,336 & 0,160 & 0,492 & 0,301 \\
\hline & 0,20 & $* * *$ & $* * *$ & $* * *$ & $* * *$ & $* * *$ & $* * *$ \\
\hline & 0,30 & $* * *$ & $* * *$ & $* * *$ & *** & $* * *$ & $* * *$ \\
\hline
\end{tabular}


Tabela 4.2.3: Binomial: poder das estatísticas $\xi_{k p}$ e $\xi_{w}$.

\begin{tabular}{|c|c|c|c|c|c|c|c|}
\hline \multicolumn{2}{|c|}{$n=50$} & \multicolumn{2}{|c|}{$\alpha=0,01$} & \multicolumn{2}{|c|}{$\alpha=0,05$} & \multicolumn{2}{|c|}{$\alpha=0,10$} \\
\hline$\rho$ & $\delta$ & $\xi_{k p}$ & $\xi_{w}$ & $\xi_{k p}$ & $\xi_{w}$ & $\xi_{k p}$ & $\xi_{w}$ \\
\hline \multicolumn{8}{|c|}{ Correlação simétrica } \\
\hline \multirow[t]{4}{*}{0,0} & 0,00 & 0,009 & 0,013 & 0,045 & 0,054 & & 0,10 \\
\hline & 0,10 & 081 & 0,054 & 0,243 & 0,148 & 0,382 & 0,251 \\
\hline & 0,20 & 0,421 & 0,299 & 0,704 & & & 0,658 \\
\hline & 0,30 & 877 & 0,764 & 0,971 & 0,927 & & 0,963 \\
\hline \multirow[t]{4}{*}{0,1} & 0,00 & 0,0 & 0,009 & 0,052 & 0,047 & 0,0 & 0,097 \\
\hline & 0,10 & 0,095 & 0,050 & 0,273 & 0,172 & 0,397 & 0,262 \\
\hline & 0,20 & 0,457 & 330 & & 0,5 & & 0,710 \\
\hline & 0,30 & 0,912 & 0,820 & 0,988 & 0,952 & & 0,982 \\
\hline \multirow[t]{4}{*}{0,3} & 0,00 & 0,009 & 0,008 & 0,056 & 0,049 & 02 & 0,109 \\
\hline & 0,10 & 0,134 & 0,074 & 0,3 & 0,209 & & \\
\hline & 0,20 & 524 & 33 & 63 & 0,730 & & 839 \\
\hline & 0,30 & 983 & 0,953 & 0,998 & 0,993 & & 0,995 \\
\hline \multirow[t]{4}{*}{0,7} & 0,00 & 016 & 0,020 & 0,063 & 0,067 & 13 & 0,123 \\
\hline & 0,10 & 337 & 0,198 & 0,656 & 0,479 & 00 & 0,641 \\
\hline & 0,20 & $* * *$ & $* * *$ & $* * *$ & $* * *$ & & $* * *$ \\
\hline & 0,30 & $* * *$ & $* * *$ & $* * *$ & *** & & $* * *$ \\
\hline \multicolumn{8}{|c|}{ Correlação AR(1) } \\
\hline \multirow[t]{4}{*}{0,0} & 0,00 & 015 & 0,012 & & 0,051 & & 0,114 \\
\hline & 0,10 & 3 & 0,060 & 0,266 & 0,17 & & 0,250 \\
\hline & 0,20 & 0,428 & 0,306 & 0,713 & 0,541 & & 688 \\
\hline & 0,30 & 0,854 & 0,771 & 0,966 & 0,919 & & 953 \\
\hline \multirow[t]{4}{*}{0,1} & 0,00 & & 0,009 & 0,067 & 0,060 & , 1 & 0,112 \\
\hline & 0,10 & & 0,063 & 0,298 & 0,179 & 0,4 & 0,284 \\
\hline & 0,20 & 0,46 & 0,319 & 0,722 & 0,574 & 826 & 0,709 \\
\hline & 0,30 & 0,875 & 0,787 & 0,971 & 0,923 & ,987 & 0,964 \\
\hline \multirow[t]{4}{*}{0,3} & 0,00 & & 0,014 & 0,065 & 0,058 &, 1 & 0,107 \\
\hline & 0,10 & 124 & 0,075 & 0,310 & 0,200 & & 0,296 \\
\hline & 0,20 & & 0,402 & 0,805 & 0,643 & 0,894 & 0,762 \\
\hline & 0,30 & 0,952 & 0,874 & 0,997 & 0,975 & 0,999 & 0,990 \\
\hline \multirow[t]{4}{*}{0,7} & 0,00 & & 0,012 & 0,087 & 0,060 & & \\
\hline & & & 0,159 & 0,592 & 0,394 & 0,718 & 0,535 \\
\hline & & & & & & & $* * *$ \\
\hline & 0,30 & 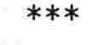 & $* * *$ & $* * *$ & $* * *$ & $* * *$ & *** \\
\hline
\end{tabular}


Tabela 4.2.4: Binomial: poder das estatísticas $\xi_{k p}$ e $\xi_{w}$.

\begin{tabular}{|c|c|c|c|c|c|c|c|}
\hline \multicolumn{2}{|c|}{$n=100$} & \multicolumn{2}{|c|}{$\alpha=0.01$} & \multicolumn{2}{|c|}{$\alpha=0,05$} & \multicolumn{2}{|c|}{$\alpha=0,10$} \\
\hline$\rho$ & $\delta$ & $\xi_{k p}$ & $\xi_{w}$ & $\xi_{k p}$ & $\xi_{w}$ & $\xi_{k p}$ & $\xi_{w}$ \\
\hline \multicolumn{8}{|c|}{ Correlação simétrica } \\
\hline \multirow[t]{4}{*}{0,0} & 0,00 & 0,012 & 0,010 & 0,047 & 0,052 & 0,095 & 0,101 \\
\hline & 0,10 & 0,188 & 0,122 & 0,433 & 0,286 & 0,567 & 0,406 \\
\hline & 0,20 & 0,789 & 0,681 & 0,935 & 0,860 & 0,969 & 0,921 \\
\hline & 0,30 & 0,998 & 0,993 & 0,999 & 0,999 & 1,000 & 1,000 \\
\hline \multirow[t]{4}{*}{0,1} & 0,00 & 0,014 & 0,013 & 0,045 & 0,055 & 0,099 & 0,110 \\
\hline & 0,10 & 0,216 & 0,128 & 0,477 & 0,322 & 0,615 & 0,453 \\
\hline & 0,20 & 0,846 & 0,749 & 0,960 & 0,903 & 0,989 & 0,953 \\
\hline & 0,30 & 0,999 & 0,996 & 1,000 & 0,999 & 1,000 & 1,000 \\
\hline \multirow[t]{4}{*}{0,3} & 0,00 & 0,010 & 0,010 & 0,059 & 0,058 & 0,106 & 0,116 \\
\hline & 0,10 & 300 & 0,204 & 0,572 & 0,401 & 0,714 & 0,545 \\
\hline & 0,20 & 922 & 0,865 & 0,986 & 0,951 & 0,994 & 0,978 \\
\hline & 0,30 & 1,000 & 1,000 & 1,000 & 1,000 & 1,000 & 1,000 \\
\hline \multirow[t]{4}{*}{0,7} & 0,00 & 0,015 & 0,015 & 0,065 & 0,060 & 0,112 & 0,113 \\
\hline & 0,10 & 742 & 0,583 & 0,920 & 0,885 & 0,962 & 0,911 \\
\hline & 0,20 & $* * *$ & $* * *$ & $* * *$ & $* * *$ & $* * *$ & $* * *$ \\
\hline & 0,30 & ** & $* * *$ & $* * *$ & $* * *$ & $* * *$ & $* * *$ \\
\hline \multicolumn{8}{|c|}{ Correlação AR(1) } \\
\hline \multirow[t]{4}{*}{0,0} & 0,00 & 0,007 & 0,007 & 0,043 & 0,036 & 0,098 & 0,081 \\
\hline & 0,10 & 0,197 & 0,109 & 0,442 & 0,297 & 0,576 & 0,436 \\
\hline & 0,20 & 0,786 & 0,675 & 0,926 & 0,853 & 0,970 & 0,914 \\
\hline & 0,30 & 0,995 & 0,994 & 0,999 & 0,998 & 1,000 & 0,999 \\
\hline \multirow[t]{4}{*}{0,1} & 0,00 & 0,006 & 0,005 & 0,042 & 0,044 & 0,108 & 0,085 \\
\hline & 0,10 & 0,205 & 0,124 & 0,466 & 0,315 & 0,621 & 0,443 \\
\hline & 0,20 & 0,814 & 0,693 & 0,937 & 0,881 & 0,975 & 0,924 \\
\hline & 0,30 & 0,997 & 0,992 & 0,999 & 0,999 & 1,000 & 0,999 \\
\hline \multirow[t]{4}{*}{0,3} & 0,00 & 0,014 & 0,008 & 0,062 & 0,053 & 0,119 & 0,101 \\
\hline & 0,10 & 0,243 & 0,150 & 0,514 & 0,346 & 0,683 & 0,487 \\
\hline & 0,20 & 0,894 & 0,797 & 0,975 & 0,923 & 0,987 & 0,965 \\
\hline & 0,30 & 1,000 & 0,998 & 1,000 & 1,000 & 1,000 & 1,000 \\
\hline \multirow[t]{4}{*}{0,7} & 0,00 & 0,013 & 0,005 & 0,081 & 0,054 & 0,143 & 0,125 \\
\hline & 0,10 & 0,597 & 0,414 & 0,835 & 0,678 & 0,904 & 0,795 \\
\hline & 0,20 & $* * *$ & $* * *$ & $* * *$ & $* * *$ & $* * *$ & $* * *$ \\
\hline & 0,30 & *** & $* * *$ & $* * *$ & *** & $* * *$ & *** \\
\hline
\end{tabular}




\subsection{Distribuição de Poisson}

Para verificar o comportamento da estatística de Kodde e Palm no caso em que as observações seguem uma distribuição de Poisson, foram geradas amostras independentes de $\boldsymbol{y}_{i}=\left(y_{i 1}, y_{i 2}, y_{i 3}\right)^{T}$ com $y_{i j} \sim \operatorname{Poisson}\left(\mu_{j}\right)$ e com estruturas de correlação simétrica e $\operatorname{AR}(1)$.

Para o caso de testar as hipóteses

$$
H_{0}: \mu_{1}=\mu_{2}=\mu_{3} \text { versus } H_{1}: \mu_{1} \leq \mu_{2} \leq \mu_{3}
$$

com pelo menos uma desigualdade estrita em $H_{1}$, foi usado o modelo $\beta_{j}=\ln \left(\mu_{j}\right)$ e as hipóteses acima são equivalentes às hipóteses (4.1.1), onde

$$
\boldsymbol{\beta}=\left(\begin{array}{c}
\beta_{1} \\
\beta_{2} \\
\beta_{3}
\end{array}\right) \text { e } \boldsymbol{R}=\left[\begin{array}{ccc}
-1 & 1 & 0 \\
0 & -1 & 1
\end{array}\right]
$$

As simulações foram conduzidas para verificar o poder da estatística de Kodde Palm levando-se em conta os valores $\mu_{1}=\mu_{2}=\mu_{3}=1$ na hipótese nula e $\mu_{1}=\mu_{2}=1$ e $\mu_{3}=1+\delta$ na hipótese alternativa para $\delta=0,0 ; 0,2 ; 0,4$; e 0,8 e valores de $\rho=0 ; 0,1$; 0,3 e 0,7 .

A Tabela 4.3.1 apresenta os resultados do teste de Kolmogorov-Smirnov (P-valor entre parênteses) para verificar o comportamento das distribuições empírica e teórica para os vários tamanhos de amostra. No caso de correlação simétrica a aproximação entre essas distribuições não é tão boa como no caso da distribuição binomial. Para $n=20$, a diferença entre essas distribuições é altamente significante. Para $n=50$ e $\rho \geq 0,3$ podemos considerar que essas distribuições não são diferentes ao nível de $5 \%$ de significância. Para $n=100$ e correlações maiores que zero não houve diferença significativa 
entre essas distribuiçōes. Com a estrutura de corelação $A R(1)$ não houve diferença significativa entre as distribuições para todos os tamanhos de amostras.

Tabela 4.3.1: Poisson: Kolmogorov-Smirnov (P-valor).

\begin{tabular}{cccc}
\hline$\rho$ & $n=20$ & $n=50$ & $n=100$ \\
\hline \multicolumn{4}{c}{ Correlação simétrica } \\
\hline \hline 0,0 & $0,1274(0,0000)$ & $0,0533(0,0551)$ & $0,0702(0,0039)$ \\
0,1 & $0,1189(0,0000)$ & $0,0642(0,0109)$ & $0,0437(0,1784)$ \\
0,3 & $0,1072(0,0000)$ & $0,0529(0,0583)$ & $0,0364(0,3723)$ \\
0,7 & $0.1181(0,0000)$ & $0,0619(0,0157)$ & $0,0425(0,2041)$ \\
\hline & Correlação AR(1) \\
\hline \hline 0,0 & $0,0333(0,4839)$ & $0,0224(0,9100)$ & $0,0187(0,9796)$ \\
0,1 & $0,0352(0,4141)$ & $0,0229(0,8950)$ & $0,0348(0,4284)$ \\
0,3 & $0,0415(0,2273)$ & $0,0221(0,9164)$ & $0,0407(0,2450)$ \\
0,7 & $0,0309(0,5804)$ & $0,0215(0,9314)$ & $0,0379(0,3234)$ \\
\hline
\end{tabular}

As Figuras 4.3.1, 4.3.2 e 4.3 .3 a seguir apresentam a distribuição empírica e a distribuição teórica dada em (4.2.1) para a estatística $\xi_{k p}$, para a estrutura de correlação simétrica entre as observações da mesma unidade experimental e amostras de tamanho 20, 50 e 100, respectivamente, considerando para cada tamanho de amostra $\rho=0,0 ; 0,1 ; 0,3$ e 0,7 . 
Figura 4.3.1: Poisson $n=20$ : distribuições empírica e teórica da estatística $\xi_{k p}$ com estrutura de correlação simétrica.
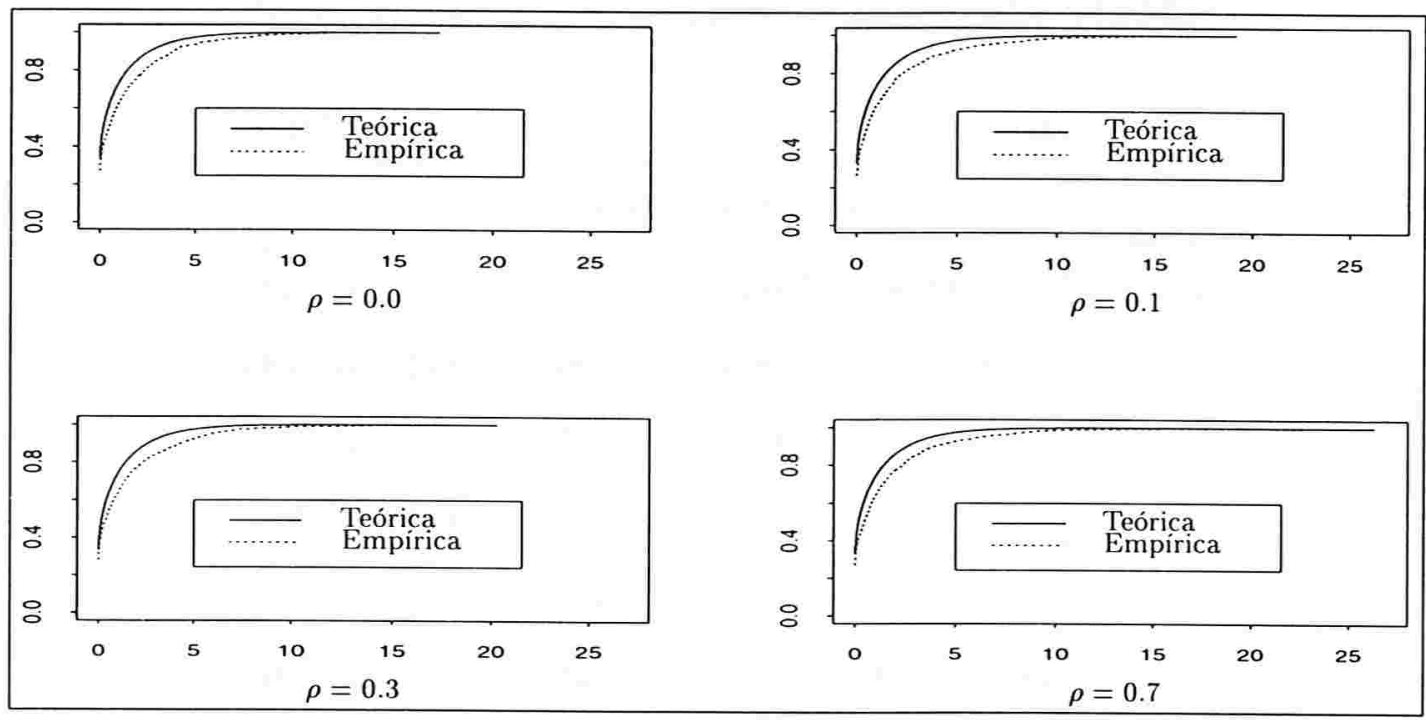

Figura 4.3.2: Poisson $n=50$ : distribuições empírica e teórica da estatística $\xi_{k p}$ com estrutura de correlação simétrica.

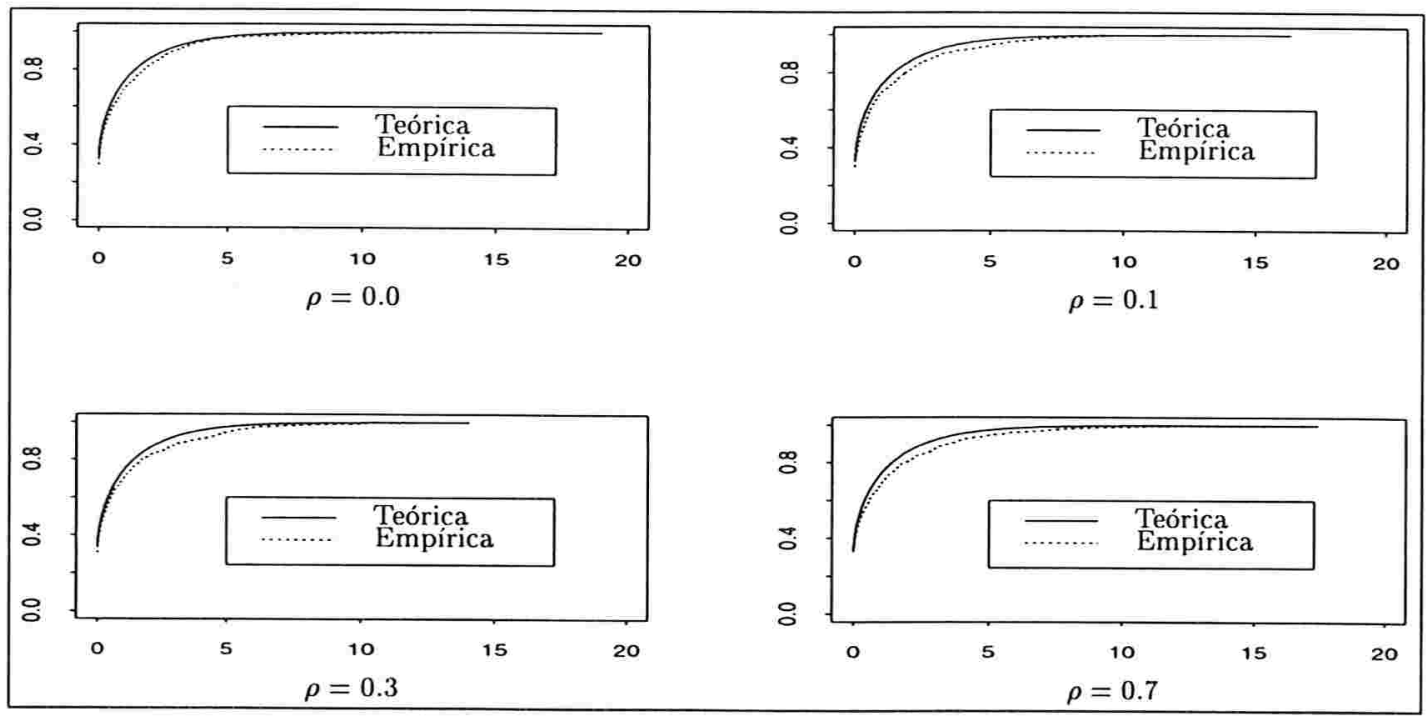


Figura 4.3.3: Poisson $n=100$ : distribuições empírica e teórica da estatística $\xi_{k p}$ com estrutura de correlação simétrica.

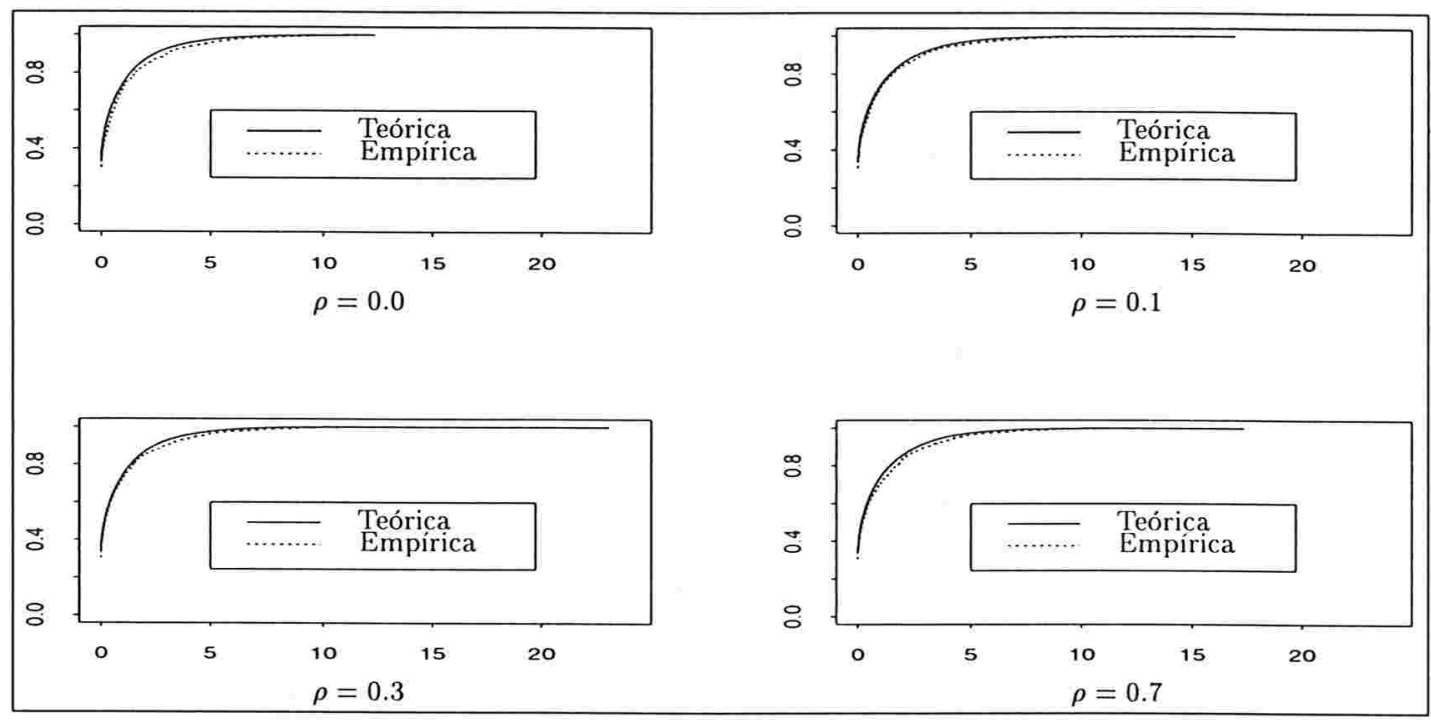

As Tabelas 4.3.2, 4.3.3 e 4.3.4 apresentam o poder das estatísticas $\xi_{k p}$ e $\xi_{w}$ para amostras de tamanhos 20, 50 e 100, respectivamente, para as estruturas de correlação simétrica e $\mathrm{AR}(1)$ e réplicas de $t=3$. Os tamanhos empíricos dos testes superestimam o valor nominal para os três tamanhos amostrais, ficando mais próximo do valor nominal para $n=100$. O poder dos testes aumenta com o tamanho amostral como também com o aumento da correlação entre as observações, como ocorre no caso binomial. É evidente a superioridade do poder do teste de Kodde e Palm em relação ao teste de Wald, contudo, o ganho de poder parece ser menor que no caso binomial. 
Tabela 4.3.2: Poisson: poder das estatísticas $\xi_{k p}$ e $\xi_{w}$.

\begin{tabular}{|c|c|c|c|c|c|c|c|}
\hline \multicolumn{2}{|c|}{$n=20$} & \multicolumn{2}{|c|}{$\alpha=0.01$} & \multicolumn{2}{|c|}{$\alpha=0,05$} & \multicolumn{2}{|c|}{$\alpha=0,10$} \\
\hline$\rho$ & $\delta$ & $\xi_{k p}$ & $\xi_{w}$ & $\xi_{k p}$ & $\xi_{w}$ & $\xi_{k p}$ & $\xi_{w}$ \\
\hline \multicolumn{8}{|c|}{ Correlação simétrica } \\
\hline \multirow[t]{4}{*}{0,0} & 0,0 & 0,035 & 0,035 & 0,101 & 0,104 & 0,176 & 0,170 \\
\hline & 0,2 & 0,076 & 0,044 & 0,181 & 0,139 & 0,272 & 0,201 \\
\hline & 0,4 & 0,176 & 0,125 & 0,357 & 0,257 & 0,474 & 0,360 \\
\hline & 0,8 & 0,537 & 0,420 & 0,747 & 0,621 & 0,834 & 0,729 \\
\hline \multirow[t]{4}{*}{0,1} & 0,0 & 0,045 & 0,035 & 0,108 & 0,103 & 0,177 & 0,154 \\
\hline & 0,2 & 0,071 & 0,051 & 0,181 & 0,138 & 0,280 & 0,212 \\
\hline & 0,4 & 0,176 & 0,130 & 0,375 & 0,269 & 0,503 & 0,369 \\
\hline & 0,8 & 0,555 & 0,453 & 0,775 & 0,663 & 0,850 & 0,762 \\
\hline \multirow[t]{4}{*}{0,3} & 0,0 & 0,037 & 0,034 & 0,120 & 0,104 & 0,183 & 0,167 \\
\hline & 0,2 & 0,074 & 0,051 & 0,187 & 0,138 & 0,293 & 0,212 \\
\hline & 0,4 & 0,229 & 0,162 & 0,456 & 0,335 & 0,584 & 0,447 \\
\hline & 0,8 & 0,681 & 0,571 & 0,860 & 0,767 & 0,918 & 0,837 \\
\hline \multirow[t]{4}{*}{0,7} & 0,0 & 0,044 & 0,035 & 0,103 & 0,105 & 0,174 & 0,166 \\
\hline & 0,2 & 0,150 & 0,103 & 0,325 & 0,235 & 0,464 & 0,331 \\
\hline & 0,4 & 0,494 & 0,361 & 0,741 & 0,607 & 0,841 & 0,716 \\
\hline & 0,8 & 0,980 & 0,947 & 0,999 & 0,990 & 1,000 & 0,999 \\
\hline \multicolumn{8}{|c|}{ Correlação AR(1) } \\
\hline \multirow[t]{4}{*}{0,0} & 0,0 & 0,018 & 0,026 & 0,076 & 0,094 & 0,124 & 0,140 \\
\hline & 0,2 & 0,071 & 0,044 & 0,181 & 0,131 & 0,278 & 0,198 \\
\hline & 0,4 & 0,184 & 0,127 & 0,363 & 0,262 & 0,493 & 0,361 \\
\hline & 0,8 & 0,562 & 0,432 & 0,763 & 0,662 & 0,858 & 0,757 \\
\hline \multirow[t]{4}{*}{0,1} & 0,0 & 0,022 & 0,021 & 0,069 & 0,068 & 0,124 & 0,105 \\
\hline & 0,2 & 0,079 & 0,050 & 0,197 & 0,134 & 0,277 & 0,191 \\
\hline & 0,4 & 0,205 & 0,144 & 0,364 & 0,281 & 0,493 & 0,357 \\
\hline & 0,8 & 0,583 & 0,465 & 0,780 & 0,668 & 0,868 & 0,771 \\
\hline \multirow[t]{4}{*}{0,3} & 0,0 & 0,023 & 0,026 & 0,065 & 0,070 & 0,112 & 0,125 \\
\hline & 0,2 & 0,069 & 0,051 & 0,197 & 0,126 & 0,308 & 0,199 \\
\hline & 0,4 & 0,223 & 0,144 & 0,402 & 0,311 & 0,534 & 0,405 \\
\hline & 0,8 & 0,626 & 0,530 & 0,815 & 0,736 & 0,894 & 0,816 \\
\hline \multirow[t]{4}{*}{0,7} & 0,0 & 0,026 & 0,028 & 0,077 & 0,066 & 0,126 & 0,117 \\
\hline & 0,2 & 0,105 & 0,078 & 0,263 & 0,187 & 0,386 & 0,272 \\
\hline & 0,4 & 0,375 & 0,284 & 0,620 & 0,510 & 0,746 & 0,631 \\
\hline & 0,8 & 0,934 & 0,891 & 0,990 & 0,972 & 0,995 & 0,992 \\
\hline
\end{tabular}


Tabela 4.3.3: Poisson: poder das estatísticas $\xi_{k p}$ e $\xi_{w}$.

\begin{tabular}{|c|c|c|c|c|c|c|c|}
\hline \multicolumn{2}{|c|}{$n=50$} & \multicolumn{2}{|c|}{$\alpha=0,01$} & \multicolumn{2}{|c|}{$\alpha=0,05$} & \multicolumn{2}{|c|}{$\alpha=0,10$} \\
\hline$\rho$ & $\delta$ & $\xi_{k p}$ & $\xi_{w}$ & $\xi_{k p}$ & $\xi_{w}$ & $\xi_{k p}$ & $\xi_{w}$ \\
\hline \multicolumn{8}{|c|}{ Correlação simétrica } \\
\hline \multirow[t]{4}{*}{0,0} & 0,0 & 0,021 & 0,018 & 0,058 & 0,061 & 0,126 & 0,099 \\
\hline & 0,2 & 0,103 & 0,069 & 0,257 & 0,174 & 0,380 & 0,270 \\
\hline & 0,4 & 0,368 & 0,256 & 0,617 & 0,481 & 0,747 & 0,596 \\
\hline & 0,8 & 0,909 & 0,864 & 0,976 & 0,951 & 0,989 & 0,978 \\
\hline \multirow[t]{4}{*}{0,1} & 0,0 & 0,022 & 0,015 & 0,084 & 0,084 & 0,138 & 0,142 \\
\hline & 0,2 & 0,114 & 0,077 & 0,275 & 0,173 & 0,408 & 0,280 \\
\hline & 0,4 & 0,392 & 0,283 & 0,658 & 0,503 & 0,757 & 0,622 \\
\hline & 0,8 & 0,930 & 0,891 & 0,980 & 0,968 & 0,990 & 0,982 \\
\hline \multirow[t]{4}{*}{0,3} & 0,0 & 0,024 & 0,025 & 0,093 & 0,076 & 0,153 & 0,151 \\
\hline & 0,2 & 0,140 & 0,089 & 0,346 & 0,214 & 0,484 & 0,333 \\
\hline & 0,4 & 0,526 & 0,395 & 0,752 & 0,632 & 0,853 & 0,745 \\
\hline & 0,8 & 0,981 & 0,960 & 0,994 & 0,989 & 0,998 & 0,994 \\
\hline \multirow[t]{4}{*}{0,7} & 0,0 & 0,031 & 0,021 & 0,087 & 0,079 & 0,154 & 0,126 \\
\hline & 0,2 & 0,307 & 0,230 & 0,554 & 0,420 & 0,696 & 0,540 \\
\hline & 0,4 & 0,902 & 0,837 & 0,973 & 0,947 & 0,991 & 0,972 \\
\hline & 0,8 & 1,000 & 1,000 & 1,000 & 1,000 & 1,000 & 1,000 \\
\hline \multicolumn{8}{|c|}{ Correlação AR(1) } \\
\hline \multirow[t]{4}{*}{0,0} & 0,0 & 0,017 & 0,010 & 0,064 & 0,051 & 0,107 & 0,111 \\
\hline & 0,2 & 0,100 & 0,058 & 0,265 & 0,167 & 0,386 & 0,258 \\
\hline & 0,4 & 0,375 & 0,276 & 0,614 & 0,469 & 0,758 & 0,597 \\
\hline & 0,8 & 0,942 & 0,888 & 0,985 & 0,967 & 0,994 & 0,987 \\
\hline \multirow[t]{4}{*}{0,1} & 0,0 & 0,012 & 0,011 & 0,054 & 0,046 & 0,101 & 0,093 \\
\hline & 0,2 & 0,105 & 0,060 & 0,270 & 0,168 & 0,382 & 0,256 \\
\hline & 0,4 & 0,399 & 0,286 & 0,638 & 0,519 & 0,762 & 0,609 \\
\hline & 0,8 & 0,936 & 0,875 & 0,984 & 0,964 & 0,994 & 0,982 \\
\hline \multirow[t]{4}{*}{0,3} & 0,0 & 0,012 & 0,008 & 0,061 & 0,057 & 0,106 & 0,107 \\
\hline & 0,2 & 0,123 & 0,073 & 0,281 & 0,185 & 0,402 & 0,278 \\
\hline & 0,4 & 0,457 & 0,381 & 0,679 & 0,572 & 0,796 & 0,683 \\
\hline & 0,8 & 0,967 & 0,927 & 0,995 & 0,980 & 1,000 & 0,996 \\
\hline \multirow[t]{4}{*}{0,7} & 0,0 & 0,013 & 0,009 & 0,056 & 0,054 & 0,108 & 0,104 \\
\hline & 0,2 & 0,216 & 0,153 & 0,454 & 0,328 & 0,594 & 0,469 \\
\hline & 0,4 & 0,800 & 0,714 & 0,932 & 0,878 & 0,968 & 0,931 \\
\hline & 0,8 & 1,000 & 1,000 & 1,000 & 1,000 & 1,000 & 1,000 \\
\hline
\end{tabular}


Tabela 4.3.4: Poisson: poder das estatísticas $\xi_{k p}$ e $\xi_{w}$.

\begin{tabular}{|c|c|c|c|c|c|c|c|}
\hline \multicolumn{2}{|c|}{$n=100$} & \multicolumn{2}{|c|}{$\alpha=0,01$} & \multicolumn{2}{|c|}{$\alpha=0,05$} & \multicolumn{2}{|c|}{$\alpha=0,10$} \\
\hline$\rho$ & $\delta$ & $\xi_{k p}$ & $\xi_{w}$ & $\xi_{k p}$ & $\xi_{w}$ & $\xi_{k p}$ & $\xi_{w}$ \\
\hline \multicolumn{8}{|c|}{ Correlação simétrica } \\
\hline \multirow[t]{4}{*}{0,0} & 0,0 & 0,018 & 0,017 & 0,070 & 0,074 & 0,131 & 0,121 \\
\hline & 0,2 & 0,177 & 0,100 & 0,399 & 0,268 & 0,552 & 0,385 \\
\hline & 0,4 & 0,691 & 0,562 & 0,870 & 0,786 & 0,938 & 0,856 \\
\hline & 0,8 & 0,997 & 0,994 & 1,000 & 0,998 & 1,000 & 1,000 \\
\hline \multirow[t]{4}{*}{0,1} & 0,0 & 0,021 & 0,014 & 0,060 & 0,072 & 0,120 & 0,123 \\
\hline & 0,2 & 0,214 & 0,142 & 0,433 & 0,296 & 0,576 & 0,412 \\
\hline & 0,4 & 0,176 & 0,130 & 0,375 & 0,269 & 0,503 & 0,369 \\
\hline & 0,8 & 1,000 & 0,999 & 1,000 & 1,000 & 1,000 & 1,000 \\
\hline \multirow[t]{4}{*}{0,3} & 0,0 & 0,021 & 0,015 & 0,072 & 0,068 & 0,128 & 0,129 \\
\hline & 0,2 & 0,283 & 0,181 & 0,518 & 0,372 & 0,652 & 0,509 \\
\hline & 0,4 & 0,869 & 0,763 & 0,958 & 0,916 & 0,982 & 0,956 \\
\hline & 0,8 & 1,000 & 1,000 & 1,000 & 1,000 & 1,000 & 1,000 \\
\hline \multirow[t]{4}{*}{0,7} & 0,0 & 0,020 & 0,015 & 0,068 & 0,069 & 0,121 & 0,128 \\
\hline & 0,2 & 0,630 & 0,505 & 0,837 & 0,727 & 0,911 & 0,807 \\
\hline & 0,4 & 0,999 & 0,997 & 1,000 & 1,000 & 1,000 & 1,000 \\
\hline & 0,8 & 1,000 & 1,000 & 1,000 & 1,000 & 1,000 & 1,000 \\
\hline \multicolumn{8}{|c|}{ Correlação AR(1) } \\
\hline \multirow[t]{4}{*}{0,0} & 0,0 & 0,013 & 0,013 & 0,064 & 0,061 & 0,115 & 0,107 \\
\hline & 0,2 & 0,194 & 0,119 & 0,424 & 0,298 & 0,553 & 0,407 \\
\hline & 0,4 & 0,699 & 0,574 & 0,878 & 0,780 & 0,938 & 0,860 \\
\hline & 0,8 & 1,000 & 0,998 & 1,000 & 1,000 & 1,000 & 1,000 \\
\hline \multirow[t]{4}{*}{0,1} & 0,0 & 0,012 & 0,009 & 0,063 & 0,057 & 0,104 & 0,098 \\
\hline & 0,2 & 0,202 & 0,123 & 0,410 & 0,289 & 0,548 & 0,392 \\
\hline & 0,4 & 0,713 & 0,585 & 0,881 & 0,790 & 0,933 & 0,867 \\
\hline & 0,8 & 0,999 & 1,000 & 1,000 & 1,000 & 1,000 & 1,000 \\
\hline \multirow[t]{4}{*}{0,3} & 0,0 & 0,013 & 0,010 & 0,055 & 0,052 & 0,108 & 0,103 \\
\hline & 0,2 & 0,232 & 0,168 & 0,432 & 0,316 & 0,571 & 0,429 \\
\hline & 0,4 & 0,767 & 0,666 & 0,922 & 0,848 & 0,974 & 0,912 \\
\hline & 0,8 & 0,999 & 0,999 & 1,000 & 1,000 & 1,000 & 1,000 \\
\hline \multirow[t]{4}{*}{0,7} & 0,0 & 0,009 & 0,008 & 0,054 & 0,051 & 0,099 & 0,106 \\
\hline & 0,2 & 0,461 & 0,342 & 0,710 & 0,589 & 0,828 & 0,717 \\
\hline & 0,4 & 0,988 & 0,973 & 0,998 & 0,993 & 0,998 & 0,997 \\
\hline & 0,8 & 1,000 & 1,000 & 1,000 & 1,000 & 1,000 & 1,000 \\
\hline
\end{tabular}




\subsection{Distribuição gama}

Para verificar o comportamento da estatística de Kodde e Palm no caso em que as observações seguem uma distribuição gama com média $\mu$, foram geradas amostras independentes de $\boldsymbol{y}_{i}=\left(y_{i 1}, y_{i 2}, y_{i 3}\right)^{T} \operatorname{com} y_{i j} \sim \operatorname{Gama}\left(\mu_{j}, \phi\right)$, para $\phi=1$ e estruturas de correlação simétrica e $\operatorname{AR}(1)$.

Para o caso de testar as hipóteses

$$
H_{0}: \mu_{1}=\mu_{2}=\mu_{3} \text { versus } H_{1}: \mu_{1} \geq \mu_{2} \geq \mu_{3}
$$

com pelo menos uma desigualdade estrita em $H_{1}$, foi usado o modelo $\beta_{j}=\mu_{j}^{-1}$ e as hipóteses acima são equivalentes às hipóteses (4.1.1), onde

$$
\boldsymbol{\beta}=\left(\begin{array}{l}
\beta_{1} \\
\beta_{2} \\
\beta_{3}
\end{array}\right) \text { e } \boldsymbol{R}=\left[\begin{array}{ccc}
-1 & 1 & 0 \\
0 & -1 & 1
\end{array}\right]
$$

As simulações foram conduzidas para verificar o poder da estatística de Kodde Palm levando-se em conta os valores $\mu_{1}=\mu_{2}=\mu_{3}=1$ para a hipótese nula e $\mu_{1}=\mu_{2}=1 \mathrm{e}$ $\mu_{3}=1-\delta$ para a hipótese alternativa, para valores de $\delta=0 ; 0,2 ; 0,4$ e 0,8 e valores de $\rho=0 ; 0,1 ; 0,3$ e 0,7 .

Os resultados do teste de Kolmogorov-Smirnov, para testar a igualdade das distribuições empírica e teórica da estatística $\xi_{k p}$ no caso em que as observações têm distribuição gama e são positivamente correlacionadas, são apresentados na Tabela 4.4.1 (P-valor entre parênteses). Para as duas estruturas de correlação, simétrica e AR(1), essas distribuições são significativamente diferentes para amostras de tamanho $n=20$. Observa-se, para correlação simétrica, que a partir de $n=50$ e $\rho=0,3$ não houve 
diferença significativa entre essas distribuições, ou seja, a aproximação entre essas distribuições é tão razoável como no caso das distribuições binomial e Poisson para grandes amostras. Com correlação $\operatorname{AR}(1)$ e $n=50$, essas distribuições são significativamente diferentes ao nível de $5 \%$ para observações altamente correlacionadas, $\rho \geq 0,3$ e não houve diferença significativa para $n=100$.

Tabela 4.4.1: Gama: Kolmogorov-Smirnov (P-valor).

\begin{tabular}{cccc}
\hline$\rho$ & $n=20$ & $n=50$ & $n=100$ \\
\hline \multicolumn{4}{c}{ Correlação simétrica } \\
\hline \hline 0,0 & $0,0607(0,0191)$ & $0,0578(0,0292)$ & $0,0274(0,7281)$ \\
0,1 & $0,0793(0,0007)$ & $0,0635(0,0121)$ & $0,0394(0,2815)$ \\
0,3 & $0,0944(0,0000)$ & $0,0439(0,1746)$ & $0,0181(0,9860)$ \\
0,7 & $0,0863(0,0002)$ & $0,0484(0,1033)$ & $0,0308(0,5862)$ \\
\hline \multicolumn{5}{c}{ Correlação AR(1) } \\
\hline \hline 0,0 & $0,0754(0,0015)$ & $0,0254(0,8114)$ & $0,0455(0,1453)$ \\
0,1 & $0,0945(0,0000)$ & $0,0307(0,5896)$ & $0,0231(0,8892)$ \\
0,3 & $0,0886(0,0001)$ & $0,0593(0,0235)$ & $0,0204(0,9554)$ \\
0,7 & $0,1085(0,0000)$ & $0,0569(0,0332)$ & $0,0392(0,2849)$ \\
\hline
\end{tabular}

As Figuras 4.4.1, 4.4.2 e 4.4 .3 a seguir apresentam a distribuição empírica e a distribuição teórica dada em (4.2.1) para a estatística $\xi_{k p}$, para a estrutura de correlação simétrica entre as observações da mesma unidade experimental e amostras de tamanho 20, 50 e 100, respectivamente, considerando para cada tamanho de amostra $\rho=0,0 ; 0,1 ; 0,3$ e 0,7 . 
Figura 4.4.1: Gama $n=20$ : distribuições empírica e teórica da estatística $\xi_{k p}$ com estrutura de correlação simétrica.

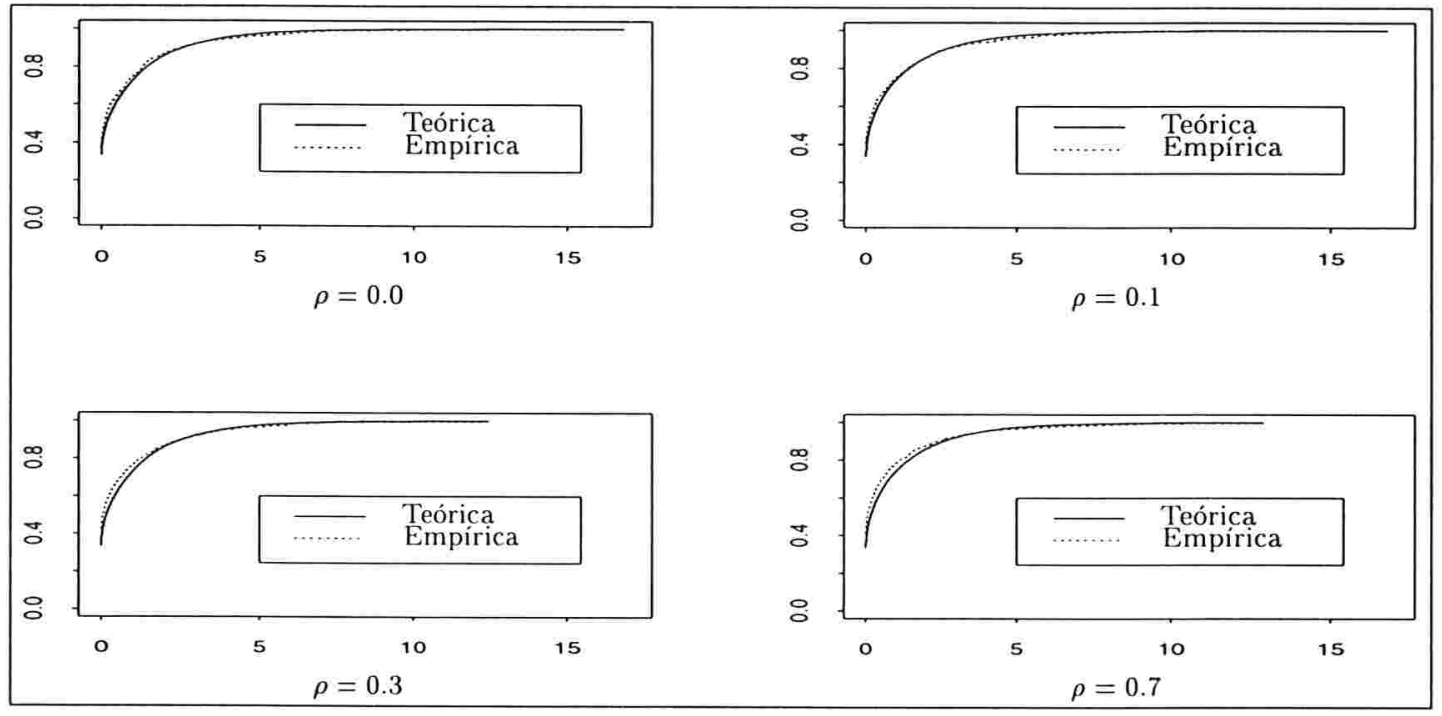

Figura 4.4.2: Gama $n=50$ : distribuições empírica e teórica da estatística $\xi_{k p}$ com estrutura de correlação simétrica.

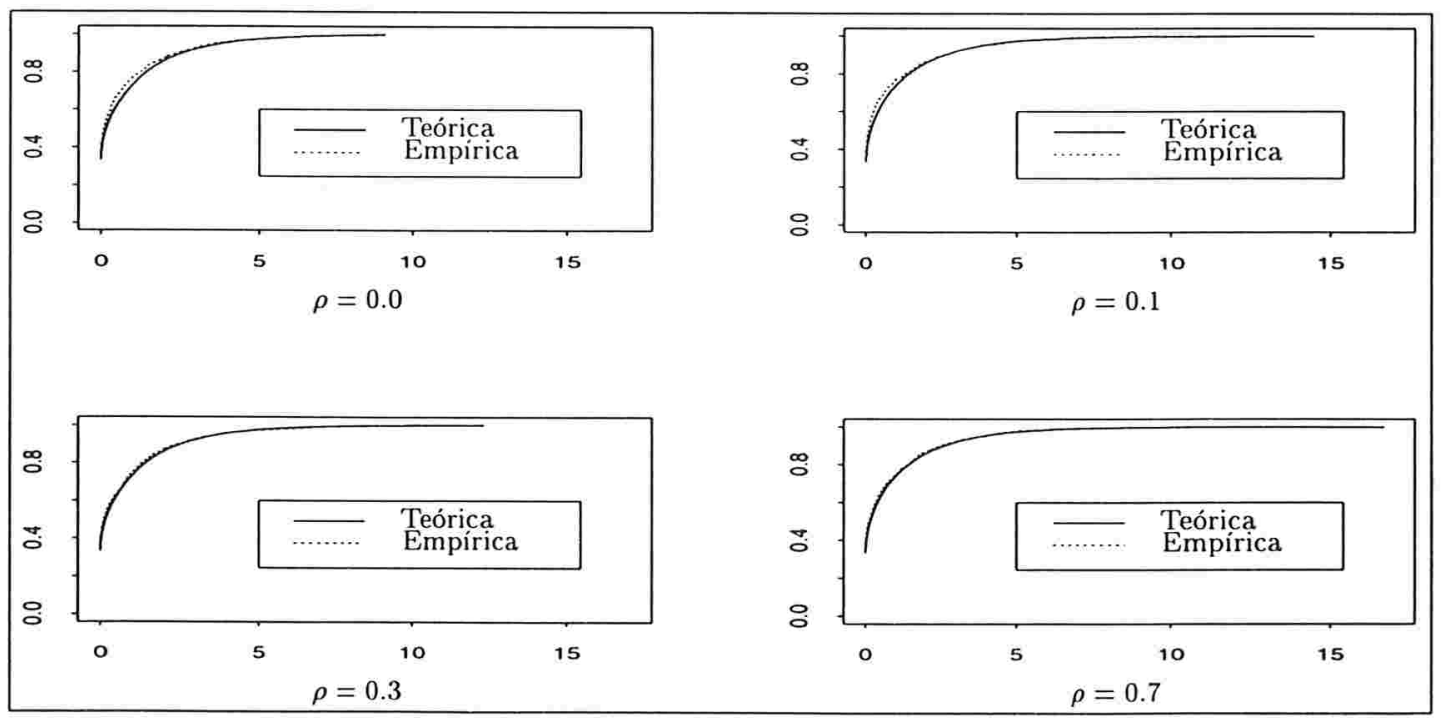


Figura 4.4.3: Gama $n=100$ : distribuições empírica e teórica da estatística $\xi_{k p}$ com estrutura de correlação simétrica.

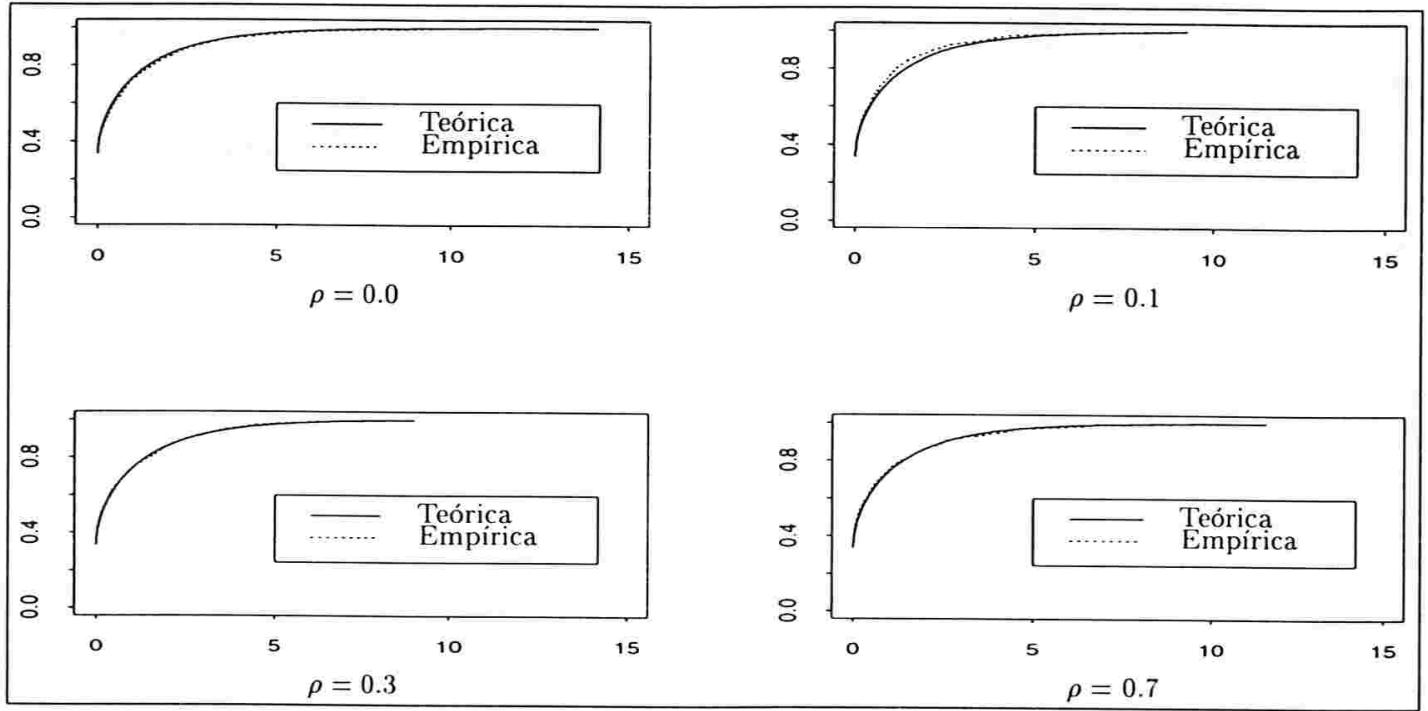

O poder estimado das estatísticas $\xi_{k p}$ e $\xi_{w}$ para observações com distribuição gama correlacionadas, é apresentado nas Tabelas 4.4.2, 4.4.3 e 4.4.4, para amostras de tamanhos 20, 50 e 100, respectivamente, com réplicas de $t=3$ e estruturas de correlação simétrica e AR(1). O tamanho empírico dos testes ficam bastante próximos do tamanho nominal exceto para correlação $\mathrm{AR}(1)$ e amostras de tamanho $n=20$. Como no caso das distribuições binomial e Poisson, o poder dessas estatísticas cresce com o tamanho da amostra e também com o aumento da correlação entre as observações da mesma unidade experimental. A estatística de Kodde e Palm apresenta um razoável ganho de poder sobre a estatística de Wald mesmo para pequenas amostras, $n=20$, em todos os níveis de significância utilizados. 
Tabela 4.4.2: Gama: poder das estatísticas $\xi_{k p}$ e $\xi_{w}$.

\begin{tabular}{|c|c|c|c|c|c|c|c|}
\hline \multicolumn{2}{|c|}{$n=20$} & \multicolumn{2}{|c|}{$\alpha=0,01$} & \multicolumn{2}{|c|}{$\alpha=0,05$} & \multicolumn{2}{|c|}{$\alpha=0,10$} \\
\hline$\rho$ & $\delta$ & $\xi_{k p}$ & $\xi_{w}$ & $\xi_{k p}$ & $\xi_{w}$ & $\xi_{k p}$ & $\xi_{w}$ \\
\hline \multicolumn{8}{|c|}{ Correlação simétrica } \\
\hline \multirow[t]{4}{*}{0,0} & 0,0 & 0,015 & 0,021 & 0,056 & 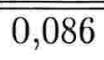 & 0,098 & 0,145 \\
\hline & 0,2 & 0,096 & 0,083 & 0,214 & 0,160 & 0,316 & 0,224 \\
\hline & 0,4 & 0,164 & 0,111 & 0,395 & 0,258 & 0,537 & 0,372 \\
\hline & 0,8 & 0,237 & 0,143 & 0,590 & 0,373 & 0,798 & 0,553 \\
\hline \multirow[t]{4}{*}{0,1} & 0,0 & 0,017 & 0,016 & 0,065 & 0,074 & 0,098 & 0,138 \\
\hline & 0,2 & 0,081 & 0,077 & 0,184 & 0,127 & 0,298 & 0,197 \\
\hline & 0,4 & 0,137 & 0,093 & 0,368 & 0,221 &, 520 & 0,336 \\
\hline & 0,8 & 0,196 & 0,124 & 0,564 & 0,319 & 0,763 & 0,502 \\
\hline \multirow[t]{4}{*}{0,3} & 0,0 & 0,014 & 0,018 & 0,053 & 0,077 & 0,098 & 0,143 \\
\hline & 0,2 & 71 & 0,060 & 0,165 & 0,120 & 0,278 & 0,187 \\
\hline & 0,4 & 0,140 & 0,088 & 0,347 & 0,225 & 0,536 & 0,344 \\
\hline & 0,8 & 0,185 & 0,094 & 0,555 & 0,303 & 0,791 & 0,478 \\
\hline \multirow[t]{4}{*}{0,7} & 0,0 & 0,020 & 0,020 & 0,051 & 0,098 & 0,089 & 0,146 \\
\hline & 0,2 & 064 & 0,042 & 0,184 & 0,100 & 309 & 0,167 \\
\hline & 0,4 & 0,159 & 0,094 & 0,515 & 0,281 & 0,722 & 0,463 \\
\hline & 0,8 & 0,236 & 0,108 & 0,651 & 0,382 & 0,844 & 0,596 \\
\hline \multicolumn{8}{|c|}{ Correlação AR(1) } \\
\hline \multirow[t]{4}{*}{0,0} & 0,0 & 0,086 & 0,125 & 0,120 & 0,162 & 0,164 & 0,210 \\
\hline & 0,2 & 0,128 & 0,112 & 0,247 & 0,177 & 0,341 & 0,244 \\
\hline & 0,4 & 0,190 & 0,137 & 0,418 & 0,276 & 0,567 & 0,406 \\
\hline & 0,8 & 0,247 & 0,184 & 0,600 & 0,382 & 0,803 & 0,559 \\
\hline \multirow[t]{4}{*}{0,1} & 0,0 & 0,104 & 0,144 & 0,143 & 0,173 & 0,188 & 0,214 \\
\hline & 0,2 & 0,124 & 0,115 & 0,224 & 0,171 & 0,314 & 0,236 \\
\hline & 0,4 & 0,204 & 0,163 & 0,391 & 0,299 & 0,548 & 0,393 \\
\hline & 0,8 & 0,243 & 0,154 & 0,618 & 0,402 & 0,840 & 0,585 \\
\hline \multirow[t]{4}{*}{0,3} & 0,0 & 0,093 & 0,135 & 0,136 & 0,189 & 0,182 & 0,246 \\
\hline & 0,2 & 0,138 & 0,120 & 0,272 & 0,202 & 0,373 & 0,282 \\
\hline & 0,4 & 0,220 & 0,179 & 0,459 & 0,327 & 0,636 & 0,444 \\
\hline & 0,8 & 0,265 & 0,190 & 0,660 & 0,423 & 0,877 & 0,616 \\
\hline \multirow[t]{4}{*}{0,7} & 0,0 & 0,089 & 0,100 & 0,133 & 0,145 & 0,191 & 0,195 \\
\hline & 0,2 & 0,137 & 0,101 & 0,313 & 0,230 & 0,464 & 0,329 \\
\hline & 0,4 & 0,298 & 0,222 & 0,660 & 0,454 & 0,822 & 0,624 \\
\hline & 0,8 & 0,344 & 0,257 & 0,710 & 0,510 & 0,888 & 0,701 \\
\hline
\end{tabular}


Tabela 4.4.3: Gama: poder das estatísticas $\xi_{k p}$ e $\xi_{w}$.

\begin{tabular}{|c|c|c|c|c|c|c|c|}
\hline \multicolumn{2}{|c|}{$n=50$} & \multicolumn{2}{|c|}{$\alpha=0,01$} & \multicolumn{2}{|c|}{$\alpha=0,05$} & \multicolumn{2}{|c|}{$\alpha=0,10$} \\
\hline$\rho$ & $\delta$ & $\xi_{k p}$ & $\xi_{w}$ & $\xi_{k p}$ & $\xi_{w}$ & $\xi_{k p}$ & $\xi_{w}$ \\
\hline \multicolumn{8}{|c|}{ Correlação simétrica } \\
\hline \multirow[t]{4}{*}{0,0} & 0,0 & 0.008 & 0,010 & 0,046 & 0,055 & 0,095 & 0,101 \\
\hline & 0,2 & 0.100 & 0,063 & 0,274 & 0,164 & 0,415 & 0,253 \\
\hline & 0,4 & 0.361 & 0,220 & 0,677 & 0,495 & 0,808 & 0,639 \\
\hline & 0,8 & 0.669 & 0,427 & 0,952 & 0,827 & 0,991 & 0,947 \\
\hline \multirow[t]{4}{*}{0,1} & 0,0 & 0.007 & 0,016 & 0,051 & 0,066 & 0,099 & 0,120 \\
\hline & 0,2 & 0.108 & 0,059 & 0,274 & 0,172 & 0,416 & 0,270 \\
\hline & 0,4 & 0.368 & 0,226 & 0,698 & 0,501 & 0,840 & 0,662 \\
\hline & 0,8 & 0.694 & 0,458 & 0,953 & 0,819 & 0,991 & 0,934 \\
\hline \multirow[t]{4}{*}{0,3} & 0,0 & 0.013 & 0,011 & 0,049 & 0,053 & 0,097 & 0,103 \\
\hline & 0,2 & 0.096 & 0,049 & 0,305 & 0,179 & 0,453 & 0,300 \\
\hline & 0,4 & 0.466 & 0,273 & 0,753 & 0,591 & 0,865 & 0,732 \\
\hline & 0,8 & 0.670 & 0,442 & 0,947 & 0,821 & 0,988 & 0,941 \\
\hline \multirow[t]{4}{*}{0,7} & 0,0 & 0.009 & 0,007 & 0,052 & 0,069 & 0,093 & 0,128 \\
\hline & 0,2 & 0.181 & 0,083 & 0,501 & 0,297 & 0,659 & 0,467 \\
\hline & 0,4 & 0.763 & 0,581 & 0,955 & 0,889 & 0,984 & 0,954 \\
\hline & 0,8 & 0.824 & 0,629 & 0,984 & 0,940 & 0,998 & 0,984 \\
\hline \multicolumn{8}{|c|}{ Correlação AR(1) } \\
\hline \multirow[t]{4}{*}{0,0} & 0,0 & 0.027 & 0,026 & 0,063 & 0,054 & 0,116 & 0,100 \\
\hline & 0,2 & 0.096 & 0,059 & 0,268 & 0,167 & 0,418 & 0,264 \\
\hline & 0,4 & 0.358 & 0,210 & 0,677 & 0,473 & 0,815 & 0,625 \\
\hline & 0,8 & 0.687 & 0,436 & 0,949 & 0,811 & 0,992 & 0,924 \\
\hline \multirow[t]{4}{*}{0,1} & 0,0 & 0.016 & 0,024 & 0,060 & 0,067 & 0,116 & 0,127 \\
\hline & 0,2 & 0.103 & 0,063 & 0,274 & 0,165 & 0,420 & 0,263 \\
\hline & 0,4 & 0.403 & 0,243 & 0,694 & 0,536 & 0,821 & 0,659 \\
\hline & 0,8 & 0.735 & 0,484 & 0,964 & 0,848 & 0,993 & 0,955 \\
\hline \multirow[t]{4}{*}{0,3} & 0,0 & 0.022 & 0,025 & 0,071 & 0,066 & 0,129 & 0,134 \\
\hline & 0,2 & 0,108 & 0,076 & 0,346 & 0,205 & 0,506 & 0,329 \\
\hline & 0,4 & 0,477 & 0,329 & 0,782 & 0,628 & 0,891 & 0,769 \\
\hline & 0,8 & 0,750 & 0,526 & 0,970 & 0,875 & 0,996 & 0,956 \\
\hline \multirow[t]{4}{*}{0,7} & 0,0 & 0,014 & 0,015 & 0,072 & 0,064 & 0,136 & 0,116 \\
\hline & 0,2 & 0.236 & 0,157 & 0,529 & 0,364 & 0,680 & 0,514 \\
\hline & 0,4 & 0,794 & 0,642 & 0,968 & 0,908 & 0,991 & 0,964 \\
\hline & 0,8 & 0,897 & 0,744 & 0,993 & 0,968 & 0,999 & 0,994 \\
\hline
\end{tabular}


Tabela 4.4.4: Gama: poder das estatísticas $\xi_{k p}$ e $\xi_{w}$.

\begin{tabular}{|c|c|c|c|c|c|c|c|}
\hline \multicolumn{2}{|c|}{$n=100$} & \multicolumn{2}{|c|}{$\alpha=0,01$} & \multicolumn{2}{|c|}{$\alpha=0,05$} & \multicolumn{2}{|c|}{$\alpha=0,10$} \\
\hline$\rho$ & $\delta$ & $\xi_{k p}$ & $\xi_{w}$ & $\xi_{k p}$ & $\xi_{w}$ & $\xi_{k p}$ & $\xi_{w}$ \\
\hline \multicolumn{8}{|c|}{ Correlação simétrica } \\
\hline \multirow[t]{4}{*}{0,0} & 0,0 & 0,015 & 0,015 & 0,055 & 0,060 & 0,105 & 0,116 \\
\hline & 0,2 & 0,185 & 0,108 & 0,444 & 0,284 & 0,589 & 0,404 \\
\hline & 0,4 & 0,732 & 0,566 & 0,937 & 0,824 & 0,980 & 0,919 \\
\hline & 0,8 & 0,990 & 0,937 & 1,000 & 0,998 & 1,000 & 1,000 \\
\hline \multirow[t]{4}{*}{0,1} & 0,0 & 0,010 & 0,008 & 0,042 & 0,048 & 0,075 & 0,096 \\
\hline & 0,2 & 0,173 & 0,101 & 0,411 & 0,253 & 0,572 & 0,373 \\
\hline & 0,4 & 0,750 & 0,590 & 0,937 & 0,846 & 0,974 & 0,919 \\
\hline & 0,8 & 0,989 & 0,947 & 0,999 & 0,995 & 1,000 & 0,999 \\
\hline \multirow[t]{4}{*}{0,3} & 0,0 & 0,008 & 0,007 & 0,046 & 0,052 & 0,102 & 0,095 \\
\hline & 0,2 & 0,231 & 0,126 & 0,495 & 0,338 & 0,644 & 0,468 \\
\hline & 0,4 & 0,858 & 0,733 & 0,975 & 0,929 & 0,990 & 0,970 \\
\hline & 0,8 & 0,991 & 0,945 & 1,000 & 0,999 & 1,000 & 1,000 \\
\hline \multirow[t]{4}{*}{0,7} & 0,0 & 0,012 & 0,007 & 0,059 & 0,051 & 0,098 & 0,098 \\
\hline & 0,2 & 0,544 & 0,381 & 0,803 & 0,664 & 0,900 & 0,781 \\
\hline & 0,4 & 0,996 & 0,979 & 1,000 & 0,999 & 1,000 & 1,000 \\
\hline & 0,8 & 0,999 & 0,994 & 1,000 & 1,000 & 1,000 & 1,000 \\
\hline \multicolumn{8}{|c|}{ Correlação AR(1) } \\
\hline \multirow[t]{4}{*}{0,0} & 0,0 & 0,015 & 0,007 & 0,074 & 0,056 & 0,131 & 0,111 \\
\hline & 0,2 & 0,199 & 0,131 & 0,444 & 0,309 & 0,571 & 0,408 \\
\hline & 0,4 & 0,757 & 0,590 & 0,920 & 0,831 & 0,959 & 0,911 \\
\hline & 0,8 & 0,990 & 0,945 & 1,000 & 0,995 & 1,000 & 1,000 \\
\hline \multirow[t]{4}{*}{0,1} & 0,0 & 0,013 & 0,014 & 0,059 & 0,057 & 0,110 & 0,106 \\
\hline & 0,2 & 0,200 & 0,111 & 0,441 & 0,294 & 0,596 & 0,412 \\
\hline & 0,4 & 0,759 & 0,601 & 0,949 & 0,855 & 0,978 & 0,936 \\
\hline & 0,8 & 0,984 & 0,949 & 1,000 & 0,995 & 1,000 & 0,998 \\
\hline \multirow[t]{4}{*}{0,3} & 0,0 & 0,017 & 0,021 & 0,062 & 0,077 & 0,114 & 0,122 \\
\hline & 0,2 & 0,247 & 0,147 & 0,511 & 0,355 & 0,666 & 0,494 \\
\hline & 0,4 & 0,855 & 0,738 & 0,968 & 0,919 & 0,985 & 0,960 \\
\hline & 0,8 & 0,993 & 0,960 & 0,999 & 0,997 & 1,000 & 0,998 \\
\hline \multirow[t]{4}{*}{0,7} & 0,0 & 0,013 & 0,008 & 0,049 & 0,045 & 0,107 & 0,102 \\
\hline & 0,2 & 0,536 & 0,404 & 0,798 & 0,657 & 0,870 & 0,778 \\
\hline & 0,4 & 0,994 & 0,984 & 0,999 & 0,997 & 1,000 & 0,999 \\
\hline & 0,8 & 1,000 & 0,998 & 1,000 & 1,000 & 1,000 & 1,000 \\
\hline
\end{tabular}




\subsection{Binomial com regressores}

Foram geradas amostras independentes de $y_{i}=\left(y_{i 1}, y_{i 2}, y_{i 3}\right)^{T}$ com $y_{i j} \sim B\left(1, \mu_{i j}\right)$, $j=1,2,3, \quad i=1,2, \ldots, n$ e com estruturas de correlação simétrica e AR(1) para as observações da mesma unidade experimental. Usando o modelo logístico linear

$$
\operatorname{logit}\left(\mu_{i j}\right)=\ln \left\{\frac{\mu_{i j}}{1-\mu_{i j}}\right\}=\beta_{0}+\beta_{1} x_{1 i}+\beta_{2} x_{2 i},
$$

$\operatorname{com} \beta_{0}=1$, simulações foram conduzidas para verificar o poder da estatística de Kodde e Palm para testar as seguintes hipóteses:

$$
H_{0}: \beta_{1}=1, \beta_{2}=1 \text { versus } H_{1}: \beta_{1} \geq 1, \beta_{2} \geq 1 \text {, }
$$

com pelo menos uma desigualdade estrita em $H_{1}$. Na hipótese alternativa foram consideados os valores de $\beta_{1}=1+\delta_{1}$ e $\beta_{2}=1+\delta_{2}$, onde $\left(\delta_{1}, \delta_{2}\right)=(0,0),(1,0 ; 1,5)$, $(1,5 ; 2,0)$, e $(2,0 ; 2,0)$ e valores de $\rho=0,0 ; 0,1 ; 0,3$ e 0,7 . As hipóteses acima são equivalentes às hipóteses

$$
H_{0}: \boldsymbol{R} \boldsymbol{\beta}=\boldsymbol{r} \text { versus } H_{1}: \boldsymbol{R} \boldsymbol{\beta} \geq \boldsymbol{r},
$$

onde

$$
\boldsymbol{\beta}=\left(\begin{array}{l}
\beta_{0} \\
\beta_{1} \\
\beta_{2}
\end{array}\right), \quad \boldsymbol{R}=\left[\begin{array}{lll}
0 & 1 & 0 \\
0 & 0 & 1
\end{array}\right] \text { e } \boldsymbol{r}=\left(\begin{array}{l}
1 \\
1
\end{array}\right) .
$$

Os resultados da simulação indicam que a aproximação entre a distribuição teórica e a distribuição empírica da estatística de Kodde e Palm não é boa mesmo para amostras de tamanho moderado. Para $n=20$ essas distribuições são significativamente diferentes para as duas estruturas de correlação. Quando $n=50$ essas distribuições não apresentam diferenças significativas, ao nível de $5 \%$, para baixas correlações, $\rho \leq 0,1 \mathrm{e}$ correlação simétrica. A diferença é significativa a $5 \%$ para correlação $\operatorname{AR}(1)$ e $\rho>0$. A 
aproximação entre essas distribuições se mostrou razoável para amostras de tamanho 100 exceto para $\rho=0,3$ com correlação simétrica e $\rho=0,7$ com correlação $\operatorname{AR}(1)$, conforme os resultados do teste de Kolmogorov-Smirnov apresentados na Tabela 4.5.1.

Tabela 4.5.1: Binomial com regressores: KolmogorovSmirnov (P-valor).

\begin{tabular}{cccc}
\hline \multicolumn{4}{c}{ Tamanho da amostra } \\
\hline$\rho$ & $n=20$ & $n=50$ & $n=100$ \\
\hline \hline \multicolumn{3}{c}{ Correlação simétrica } \\
\hline \hline 0,0 & $0,1210(0,0000)$ & $0,0436(0,1818)$ & $0,0218(0,9246)$ \\
0,1 & $0,1282(0,0000)$ & $0,0508(0,0770)$ & $0,0376(0,3336)$ \\
0,3 & $0,1217(0,0000)$ & $0,0726(0,0026)$ & $0,0561(0,0374)$ \\
0,7 & $0,0828(0,0003)$ & $0,0932(0,0000)$ & $0,0475(0,1162)$ \\
\hline & & Correlação AR(1) \\
\hline \hline 0,0 & $0,1190(0,0000)$ & $0,0467(0,1265)$ & $0,0218(0,9239)$ \\
0,1 & $0,1259(0,0000)$ & $0,0596(0,0224)$ & $0,0413(0,2306)$ \\
0,3 & $0,1360(0,0000)$ & $0,0760(0,0013)$ & $0,0384(0,3087)$ \\
0,7 & $0,1577(0,0000)$ & $0,0892(0,0001)$ & $0,0550(0,0439)$ \\
\hline
\end{tabular}

As Figuras 4.5.1, 4.5.2 e 4.5.3 a seguir mostram, para a estatística $\xi_{k p}$, a distribuição empírica e a distribuição teórica dada em (4.2.1) para estrutura de correlação simétrica e todos os valores de $\rho$ considerados e amostras de tamanho 20, 50 e 100, respectivamente. 
Figura 4.5.1: Binomial com regressores $n=20$ : distribuições empírica e teórica da estatística $\xi_{k p}$ com estrutura de correlação simétrica.

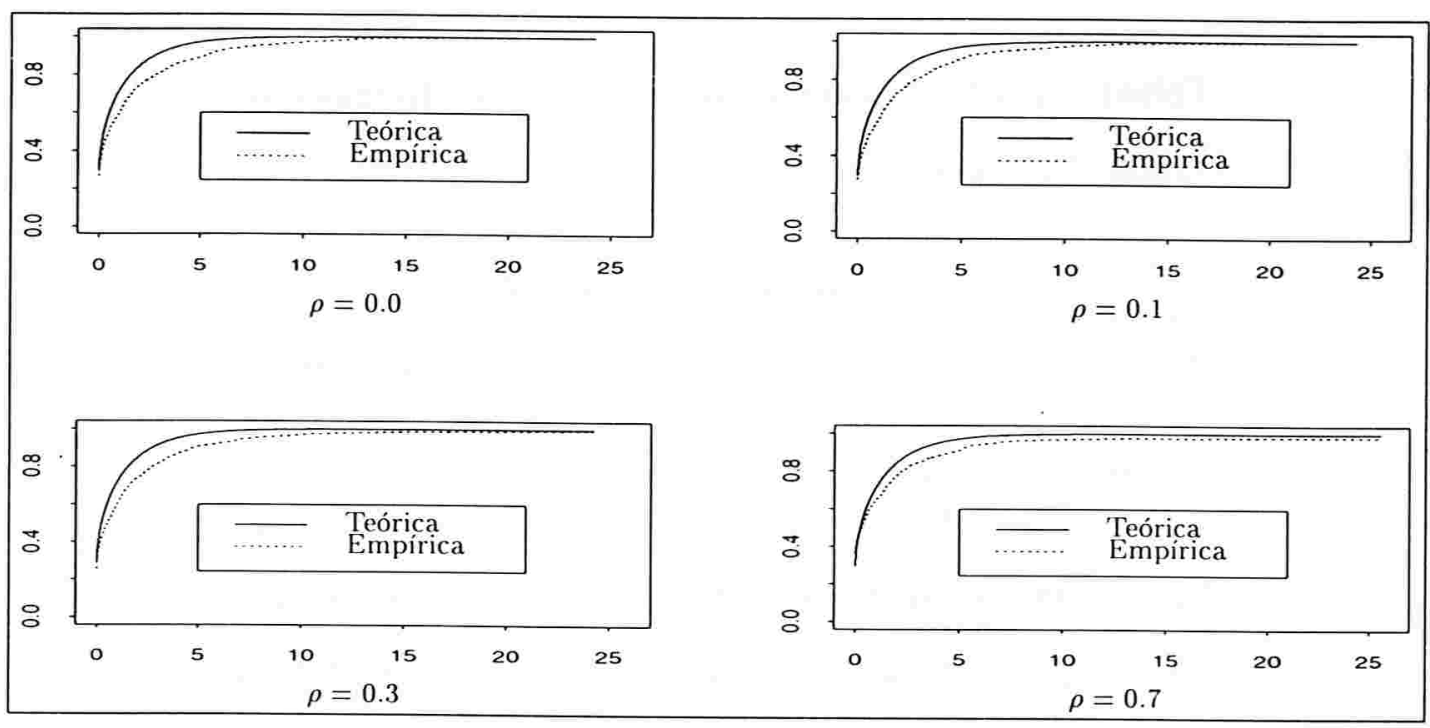

Figura 4.5.2: Binomial com regressores $n=50$ : distribuições empírica e teórica da estatística $\xi_{k p}$ com estrutura de correlação simétrica.

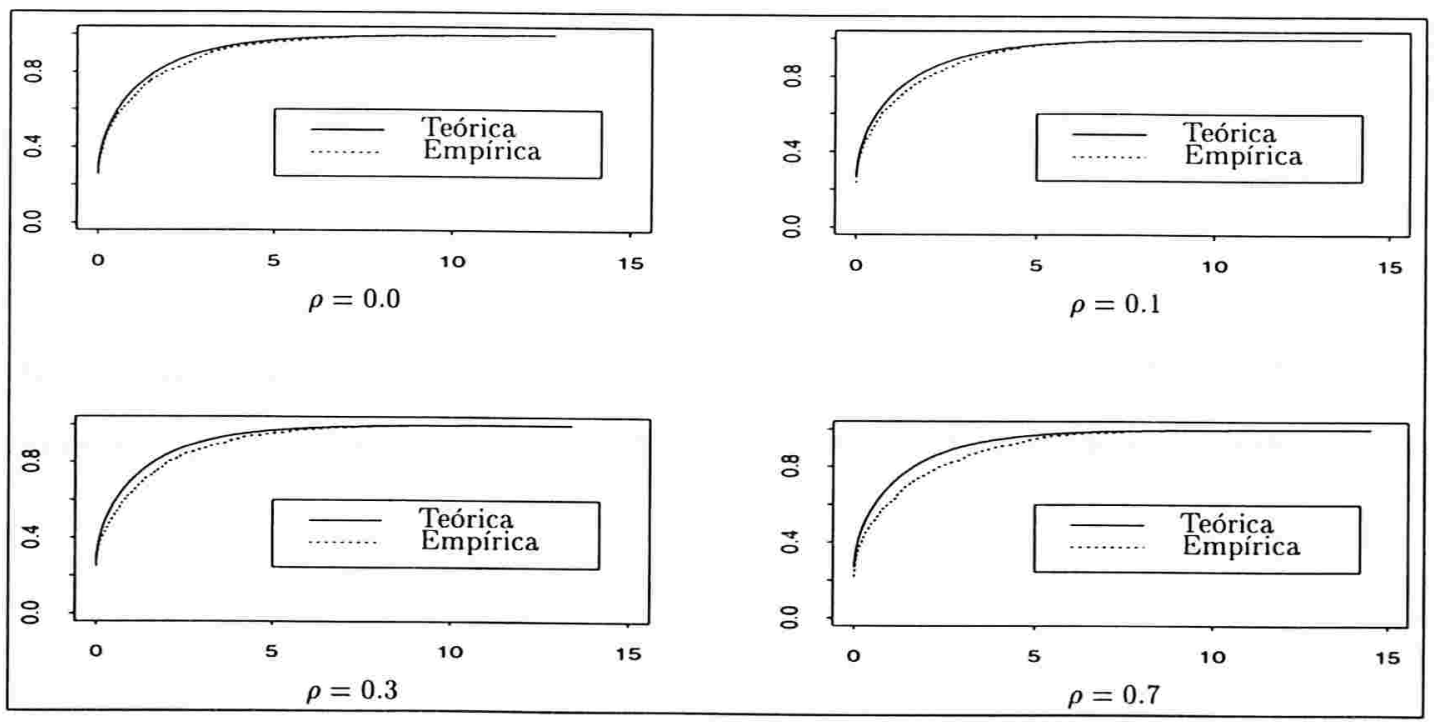


Figura 4.5.3: Binomial com regressores $n=100$ : distribuições empírica e teórica da estatística $\xi_{k p}$ com estrutura de correlação simétrica.

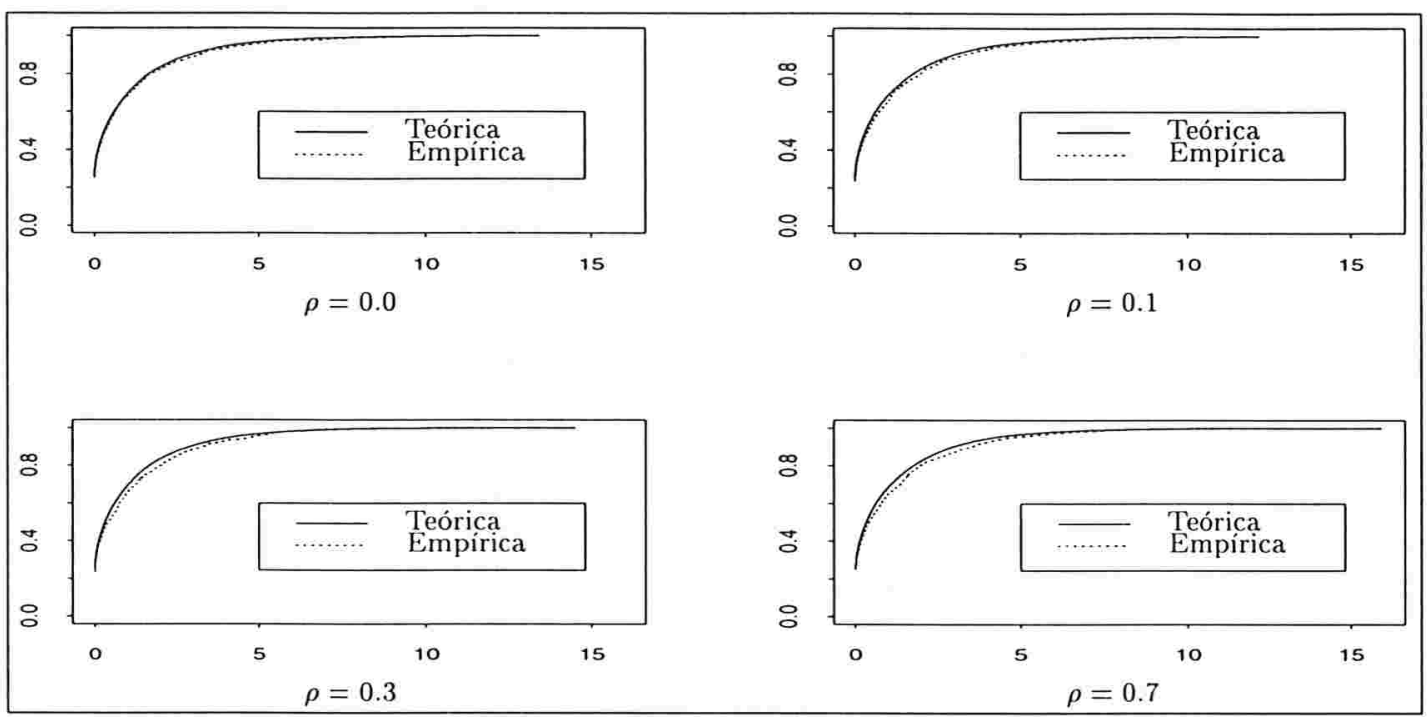

As Tabelas 4.5.2, 4.5.3 e 4.5.4 apresentam o poder estimado da estatística de Kodde e Palm e da estatística de Wald com estrutura de correlação simétrica e AR(1), para amostras de tamanho 20, 50 e 100, respectivamente. Nota-se para $n=20$ que os tamanhos empíricos dos testes $\xi_{k p}$ e $\xi_{w}$ são superiores ao tamanho nominal. Para $n$ $=50$ e $n=100$ os tamanhos empíricos ficam mais próximos do valor nominal. Em geral, a diferença entre o tamanho empírico e o tamanho nominal dos testes aumenta com o aumento da correlação entre as observações. Nota-se também que o poder dos testes diminui com o aumento da correlação entre as observações. Isso é explicado pelo fato de as observações $y_{i j}(j=1,2,3)$ não conterem muita informação se forem altamente correlacionadas (vide Apêndice C). É evidente a superioridade da estatística de Kodde e Palm sobre a estatística de Wald, particularmente para $n=50$ e $n=100$. O comportamento do poder estimado para os dois testes é semelhante para as duas estruturas de correlaçao. 
Tabela 4.5.2: Binomial com regressores: poder das estatísticas $\xi_{k p}$ e $\xi_{w}$.

\begin{tabular}{|c|c|c|c|c|c|c|c|c|}
\hline \multicolumn{3}{|c|}{$n=20$} & \multicolumn{2}{|c|}{$\alpha=0,01$} & \multicolumn{2}{|c|}{$\alpha=0,05$} & \multicolumn{2}{|c|}{$\alpha=0,10$} \\
\hline$\rho$ & $\delta_{1}$ & $\delta_{2}$ & $\xi_{k p}$ & $\xi_{w}$ & $\xi_{k p}$ & $\xi_{w}$ & $\xi_{k p}$ & $\xi_{w}$ \\
\hline \multicolumn{9}{|c|}{ Correlação simétrica } \\
\hline \multirow[t]{4}{*}{0,0} & 0,0 & 0,0 & 0,057 & 0,080 & 0,135 & 0,168 & 0,202 & 0,255 \\
\hline & 1,0 & 1,5 & 0,236 & 0,248 & 0,435 & 0,378 & 0,501 & 0,488 \\
\hline & 1,5 & 2,0 & 0,271 & 0,305 & 0,521 & 0,431 & 0,580 & 0,569 \\
\hline & 2,0 & 2,0 & 0,301 & 0,328 & 0,545 & 0,452 & 0,599 & 0.587 \\
\hline \multirow[t]{4}{*}{0,1} & 0,0 & 0,0 & 0,050 & 0,071 & 0,128 & 0,162 & 0,196 & 0.244 \\
\hline & 1,0 & 1,5 & 0,224 & 0,239 & 0,422 & 0,363 & 0,493 & 0.471 \\
\hline & 1,5 & 2,0 & 0,386 & 0,303 & 0,615 & 0,468 & 0,730 & 0,580 \\
\hline & 2,0 & 2,0 & 0.417 & 0,320 & 0,630 & 0,490 & 0,744 & 0.596 \\
\hline \multirow[t]{4}{*}{0,3} & 0,0 & 0,0 & 0,058 & 0,081 & 0,125 & 0,169 & 0,198 & 0.240 \\
\hline & 1,0 & 1,5 & 0,201 & 0,239 & 0,393 & 0,350 & 0,453 & 0,465 \\
\hline & 1,5 & 2,0 & 0,313 & 0,242 & 0,532 & 0,382 & 0,641 & 0,504 \\
\hline & 2,0 & 2,0 & 0,327 & 0,261 & 0,553 & 0,408 & 0,677 & 0.531 \\
\hline \multirow[t]{5}{*}{0,7} & 0,0 & 0,0 & 0,045 & 0,065 & 0,117 & 0,157 & 0,161 & 0,238 \\
\hline & 1,0 & 1,5 & 0,139 & 0,165 & 0,263 & 0,236 & 0,293 & 0,317 \\
\hline & 1,5 & 2,0 & 0,200 & 0,169 & 0,382 & 0,282 & 0,479 & 0,379 \\
\hline & 2,0 & 2,0 & 0,207 & 0,185 & 0,398 & 0,300 & 0,493 & 0,398 \\
\hline & \multicolumn{8}{|c|}{ Correlação AR(1) } \\
\hline \multirow[t]{4}{*}{0,0} & 0,0 & 0,0 & 0,060 & 0,080 & 0,137 & 0,174 & 0,205 & 0,255 \\
\hline & 1,0 & 1,5 & 0,238 & 0,248 & 0,437 & 0,380 & 0,501 & 0,492 \\
\hline & 1,5 & 2,0 & 0,274 & 0,305 & 0,523 & 0,434 & 0,581 & 0,567 \\
\hline & 2,0 & 2,0 & 0,305 & 0,329 & 0,548 & 0,456 & 0,602 & 0,592 \\
\hline \multirow[t]{4}{*}{0,1} & 0,0 & 0,0 & 0,053 & 0,077 & 0,134 & 0,174 & 0,212 & 0,252 \\
\hline & 1,0 & 1,5 & 0,225 & 0,243 & 0,431 & 0,369 & 0,499 & 0,482 \\
\hline & 1,5 & 2,0 & 0,275 & 0,308 & 0,517 & 0,438 & 0,567 & 0,570 \\
\hline & 2,0 & 2,0 & 0,298 & 0,325 & 0,535 & 0,443 & 0,582 & 0,586 \\
\hline \multirow[t]{4}{*}{0,3} & 0,0 & 0,0 & 0,068 & 0,097 & 0,146 & 0,186 & 0,213 & 0,2262 \\
\hline & 1,0 & 1,5 & 0,226 & 0,258 & 0,429 & 0,388 & 0,490 & 0,513 \\
\hline & 1,5 & 2,0 & 0,259 & 0,295 & 0,485 & 0,405 & 0,536 & 0,546 \\
\hline & 2,0 & 2,0 & 0,280 & 0,308 & 0,503 & 0,420 & 0,543 & 0,548 \\
\hline \multirow[t]{4}{*}{0,7} & 0,0 & 0,0 & 0,080 & 0,108 & 0,163 & 0,200 & 0,224 & 0,304 \\
\hline & 1,0 & 1,5 & 0,230 & 0,281 & 0,406 & 0,376 & 0,451 & 0,500 \\
\hline & 1,5 & 2,0 & 0,241 & 0,267 & 0,401 & 0,352 & 0,434 & 0,458 \\
\hline & 2,0 & 2,0 & 0,250 & 0,269 & 0,409 & 0,349 & 0,431 & 0,449 \\
\hline
\end{tabular}


Tabela 4.5.3: Binomial com regressores: poder das estatísticas $\xi_{k p}$ e $\xi_{w}$.

\begin{tabular}{|c|c|c|c|c|c|c|c|c|}
\hline \multicolumn{3}{|c|}{$n=50$} & \multicolumn{2}{|c|}{$\alpha=0,01$} & \multicolumn{2}{|c|}{$\alpha=0,05$} & \multicolumn{2}{|c|}{$\alpha=0,10$} \\
\hline$\rho$ & $\delta_{1}$ & $\delta_{2}$ & $\xi_{k p}$ & $\xi_{w}$ & $\xi_{k p}$ & $\xi_{w}$ & $\xi_{k p}$ & $\xi_{w}$ \\
\hline \multicolumn{9}{|c|}{ Correlação simétrica } \\
\hline \multirow[t]{4}{*}{0,0} & 0,0 & 0,0 & $\overline{0,014}$ & 0,016 & 0,061 & 0,075 & 0,123 & 0,126 \\
\hline & 1,0 & 1,5 & 0,186 & 0,127 & 0.412 & 0,271 & 0,549 & 0,401 \\
\hline & 1,5 & 2,0 & 0,283 & 0,210 & 0,528 & 0,377 & 0,669 & 0,499 \\
\hline & 2,0 & 2,0 & 0,362 & 0,261 & 0.595 & 0,441 & 0,729 & 0,581 \\
\hline \multirow[t]{4}{*}{0,1} & 0,0 & 0,0 & 0,009 & 0,021 & 0,060 & 0,067 & 0,127 & 0,128 \\
\hline & 1,0 & 1,5 & 0,181 & 0,121 & 0.403 & 0,268 & 0,536 & 0,391 \\
\hline & 1,5 & 2,0 & 0,285 & 0,204 & 0.528 & 0,382 & 0,656 & 0,507 \\
\hline & 2,0 & 2,0 & 0,348 & 0,253 & 0.580 & 0,423 & 0,716 & 0,562 \\
\hline \multirow[t]{4}{*}{0,3} & 0,0 & 0,0 & 0,015 & 0,018 & 0.068 & 0,080 & 0,138 & 0,145 \\
\hline & 1,0 & 1.5 & 0,169 & 0,118 & 0.384 & 0,262 & 0,517 & 0,377 \\
\hline & 1,5 & 2,0 & 0,281 & 0,207 & 0,515 & 0,381 & 0,652 & 0,502 \\
\hline & 2,0 & 2,0 & 0,340 & 0,254 & 0,556 & 0,429 & 0,701 & 0,536 \\
\hline \multirow[t]{4}{*}{0,7} & 0,0 & 0,0 & 0,017 & 0,027 & 0.085 & 0,099 & 0,151 & 0,180 \\
\hline & 1,0 & 1,5 & 0,178 & 0,142 & 0,359 & 0,279 & 0,468 & 0,372 \\
\hline & 1,5 & 2,0 & 0,246 & 0,214 & 0,451 & 0,361 & 0,555 & 0,465 \\
\hline & 2,0 & 2,0 & 0,286 & 0,236 & 0,449 & 0,376 & 0,559 & 0,468 \\
\hline \multicolumn{9}{|c|}{ Correlação AR(1) } \\
\hline \multirow[t]{4}{*}{0,0} & 0,0 & 0,0 & 0,012 & 0,018 & 0,063 & 0,077 & 0,124 & 0,127 \\
\hline & 1,0 & 1,5 & 0,190 & 0,129 & 0,415 & 0,275 & 0,543 & 0,403 \\
\hline & 1,5 & 2,0 & 0,280 & 0,208 & 0,532 & 0,381 & 0,670 & 0,493 \\
\hline & 2,0 & 2,0 & 0,358 & 0,265 & 0.601 & 0,441 & 0,729 & 0,579 \\
\hline \multirow[t]{4}{*}{0,1} & 0,0 & 0,0 & 0,011 & 0,023 & 0,057 & 0,069 & 0,125 & 0,118 \\
\hline & 1,0 & 1,5 & 0,186 & 0,124 & 0,405 & 0,276 & 0,539 & 0,390 \\
\hline & 1,5 & 2,0 & 0,295 & 0,206 & 0,528 & 0,394 & 0,661 & 0,505 \\
\hline & 2,0 & 2,0 & 0,353 & 0,267 & 0.584 & 0,431 & 0,719 & 0,568 \\
\hline \multirow{4}{*}{0,3} & 0,0 & 0,0 & 0,012 & 0,018 & 0.072 & 0,081 & 0,142 & 0,149 \\
\hline & 1,0 & 1,5 & 0,176 & 0,122 & 0,381 & 0,273 & 0,518 & 0,372 \\
\hline & 1,5 & 2,0 & 0,289 & 0,214 & 0,524 & 0,385 & 0,651 & 0,503 \\
\hline & 2,0 & 2,0 & 0,351 & 0,262 & 0,581 & 0,441 & 0,701 & 0,552 \\
\hline \multirow[t]{4}{*}{0,7} & 0,0 & 0,0 & 0,017 & 0,028 & 0,085 & 0,087 & 0,149 & 0,164 \\
\hline & 1,0 & 1,5 & 0,216 & 0,147 & 0,400 & 0,315 & 0,528 & 0,418 \\
\hline & 1,5 & 2,0 & 0,302 & 0,244 & 0,504 & 0,420 & 0,637 & 0,525 \\
\hline & 2,0 & 2,0 & 0,363 & 0,290 & 0,561 & 0,476 & 0,680 & 0,563 \\
\hline
\end{tabular}


Tabela 4.5.4: Binomial com regressores: poder das estatísticas $\xi_{k p}$ e $\xi_{w}$.

\begin{tabular}{|c|c|c|c|c|c|c|c|c|}
\hline \multicolumn{3}{|c|}{$n=100$} & \multicolumn{2}{|c|}{$\alpha=0,01$} & \multicolumn{2}{|c|}{$\alpha=0,05$} & \multicolumn{2}{|c|}{$\alpha=0,10$} \\
\hline$\rho$ & $\delta_{1}$ & $\delta_{2}$ & $\xi_{k p}$ & $\xi_{w}$ & $\xi_{k p}$ & $\xi_{w}$ & $\xi_{k p}$ & $\xi_{w}$ \\
\hline \multicolumn{9}{|c|}{ Correlação simétrica } \\
\hline \multirow[t]{4}{*}{0,0} & 0,0 & 0,0 & 0,016 & 0,020 & 0,060 & 0,067 & 0,118 & 0,117 \\
\hline & 1,0 & 1,5 & 0,271 & 0,148 & 0,549 & 0,360 & 0,679 & 0,488 \\
\hline & 1,5 & 2,0 & 0,362 & 0,235 & 0,659 & 0,473 & 0,774 & 0,626 \\
\hline & 2,0 & 2,0 & 0,420 & 0,309 & 0,696 & 0,528 & 0,800 & 0,669 \\
\hline \multirow[t]{4}{*}{0,1} & 0,0 & 0,0 & 0,016 & 0,023 & 0,058 & 0,069 & 0,118 & 0,124 \\
\hline & 1,0 & 1,5 & 0,251 & 0,135 & 0,518 & 0,333 & 0,665 & 0,469 \\
\hline & 1,5 & 2,0 & 0,347 & 0,220 & 0,615 & 0,454 & 0,762 & 0,586 \\
\hline & 2.0 & 2,0 & 0,397 & 0,284 & 0,662 & 0,514 & 0,783 & 0,635 \\
\hline \multirow[t]{4}{*}{0,3} & 0,0 & 0,0 & 0,007 & 0,018 & 0,069 & 0,071 & 0,120 & 0,137 \\
\hline & 1,0 & 1,5 & 0,228 & 0,135 & 0,493 & 0,317 & 0,627 & 0,449 \\
\hline & 1,5 & 2,0 & 0,327 & 0,215 & 0,586 & 0,436 & 0,718 & 0,566 \\
\hline & 2,0 & 2,0 & 0,370 & 0,258 & 0,634 & 0,468 & 0,767 & 0,601 \\
\hline \multirow[t]{5}{*}{0,7} & 0,0 & 0,0 & 0,015 & 0,022 & 0,068 & 0,078 & 0,133 & 0,129 \\
\hline & 1,0 & 1,5 & 0,189 & 0,115 & 0,410 & 0,283 & 0,563 & 0,385 \\
\hline & 1,5 & 2,0 & 0,277 & 0,204 & 0,528 & 0,397 & 0,650 & 0,522 \\
\hline & 2,0 & 2,0 & 0,315 & 0,250 & 0,568 & 0,427 & 0,672 & 0,541 \\
\hline & \multicolumn{8}{|c|}{ Correlação AR(1) } \\
\hline \multirow[t]{4}{*}{0,0} & 0,0 & 0,0 & 0,017 & 0,021 & 0,060 & 0,068 & 0,119 & 0,118 \\
\hline & 1,0 & 1,5 & 0,251 & 0,149 & 0,520 & 0,357 & 0,662 & 0,490 \\
\hline & 1,5 & 2,0 & 0,359 & 0,236 & 0,657 & 0,469 & 0,773 & 0,628 \\
\hline & 2,0 & 2,0 & 0,422 & 0,310 & 0,697 & 0,527 & 0,802 & 0,667 \\
\hline \multirow[t]{4}{*}{0,1} & 0,0 & 0,0 & 0,017 & 0,025 & 0,067 & 0,066 & 0,116 & 0,122 \\
\hline & 1,0 & 1,5 & 0,239 & 0,140 & 0,499 & 0,335 & 0,640 & 0,472 \\
\hline & 1,5 & 2,0 & 0,347 & 0,222 & 0,625 & 0,457 & 0,764 & 0,588 \\
\hline & 2,0 & 2,0 & 0,402 & 0,284 & 0,667 & 0,502 & 0,785 & 0,628 \\
\hline \multirow[t]{4}{*}{0,3} & 0,0 & 0,0 & 0,009 & 0,017 & 0,067 & 0,071 & 0,129 & 0,129 \\
\hline & 1,0 & 1,5 & 0,223 & 0,136 & 0,469 & 0,320 & 0,606 & 0,454 \\
\hline & 1,5 & 2,0 & 0,332 & 0,226 & 0,584 & 0,430 & 0,718 & 0,554 \\
\hline & 2,0 & 2,0 & 0,360 & 0,252 & 0,633 & 0,461 & 0,757 & 0,605 \\
\hline \multirow[t]{4}{*}{0,7} & 0,0 & 0,0 & 0,019 & 0,028 & 0,062 & 0,074 & 0,116 & 0,128 \\
\hline & 1,0 & 1,5 & 0,174 & 0,119 & 0,397 & 0,276 & 0,545 & 0,382 \\
\hline & 1,5 & 2,0 & 0,290 & 0,202 & 0,533 & 0,393 & 0,657 & 0,509 \\
\hline & 2,0 & 2,0 & 0,331 & 0,242 & 0,582 & 0,429 & 0,712 & 0,551 \\
\hline
\end{tabular}




\subsection{Poisson com regressores}

Amostras independentes de $y_{i}=\left(y_{i 1}, y_{i 2}, y_{i 3}, y_{i 4}\right)^{T} \operatorname{com} y_{i j} \sim \operatorname{Poisson}\left(\mu_{i j}\right), j=1,2,3,4$, $i=1,2, \ldots, n$, foram geradas com estruturas de correlação simétrica e AR(1) para as observações da mesma unidade experimental.

Usando o modelo de regressão de Poisson

$$
\ln \left(\mu_{i j}\right)=\beta_{0}+\beta_{1} x_{1 i}+\beta_{2} x_{2 i}
$$

$\operatorname{com} \beta_{0}=1$, simulações foram conduzidas para verificar o poder da estatística de Kodde e Palm para testar as seguintes hipóteses:

$$
H_{0}: \beta_{1}=1, \beta_{2}=1 \text { versus } H_{1}: \beta_{1} \geq 1, \beta_{2} \geq 1
$$

com pelo menos uma desigualdade estrita em $H_{1}$. Na hipótese alternativa foram considerados $\beta_{1}=1+\delta_{1}$ e $\beta_{2}=1+\delta_{2}$ para valores de $\left(\delta_{1}, \delta_{2}\right)=(0 ; 0),(0 ; 0,2),(0,2 ; 0)$ e $(0,2 ; 0,2)$ e valores de $\rho=0 ; 0,1 ; 0,3$ e 0,7 . As hipóteses acima são equivalentes às hipóteses (4.5.1) com

$$
\boldsymbol{\beta}=\left(\begin{array}{c}
\beta_{0} \\
\beta_{1} \\
\beta_{2}
\end{array}\right), \quad \boldsymbol{R}=\left[\begin{array}{lll}
0 & 1 & 0 \\
0 & 0 & 1
\end{array}\right] \text { e } \boldsymbol{r}=\left(\begin{array}{l}
1 \\
1
\end{array}\right) .
$$

Os resultados da simulação indicam que a aproximação entre as distribuições teórica e empirica, para estrutura de correlação $\operatorname{AR}(1)$, não é boa mesmo para amostras de tamanho moderado. Essa aproximação se mostrou razoável somente para amostra de tamanho $n=50$ e $\rho=0$ e amostras de tamanho $n=100$ para $\rho \geq 0,3$. Para a estrutura de correlação simétrica a aproximação entre as distribuições empírica e teórica foi muito boa para $n=50$, enquanto que para os outros tamanhos de amostra a aproximação 
entre as distribuições foi semelhante àquela encontrada para correlação $\mathrm{AR}(1)$, conforme os resultados do teste de Kolmogorov-Smirnov apresentados na Tabela 4.6.1.

Tabela 4.6.1: Poisson com regressores: Kolmogorov-

Smirnov (P-valor).

\begin{tabular}{cccc}
\hline \multicolumn{4}{c}{ Tamanho da amostra } \\
\hline$\rho$ & $n=20$ & $n=50$ & $n=100$ \\
\hline \multicolumn{4}{c}{ Correlação simétrica } \\
\hline \hline 0,0 & $0,0903(0,0001)$ & $0,0377(0,3294)$ & $0,0799(0,0006)$ \\
0,1 & $0,1110(0,0000)$ & $0,0458(0.1409)$ & $0,0882(0,0001)$ \\
0,3 & $0,0759(0,0014)$ & $0,0301(0,6159)$ & $0,0413(0,2310)$ \\
0,7 & $0,0710(0,0034)$ & $0,0407(0,2456)$ & $0,0464(0,1308)$ \\
\hline \multicolumn{5}{c}{ Correlação AR(1) } \\
\hline \hline 0,0 & $0,0931(0,0000)$ & $0,0374(0,3401)$ & $0,0789(0,0008)$ \\
0,1 & $0,1378(0,0000)$ & $0,0541(0,0498)$ & $0,0944(0,0000)$ \\
0,3 & $0,1004(0,0000)$ & $0,0582(0,0277)$ & $0,0279(0,7085)$ \\
0,7 & $0,1005(0,0000)$ & $0,0687(0,0051)$ & $0,0263(0,7764)$ \\
\hline
\end{tabular}

As Figuras 4.6.1, 4.6.2 e 4.6.3 a seguir mostram, para a estatística $\xi_{k p}$, a distribuição empírica e a distribuição teórica dada em (4.2.1) para estrutura de correlação AR(1) e amostras de tamanho 20, 50 e 100, respectivamente, para todos os valores de $\rho$ considerados. 
Figura 4.6.1: Poisson com regressores $n=20$ : distribuições empírica e teórica da estatística $\xi_{k p}$ com estrutura de correlação $\operatorname{AR}(1)$.
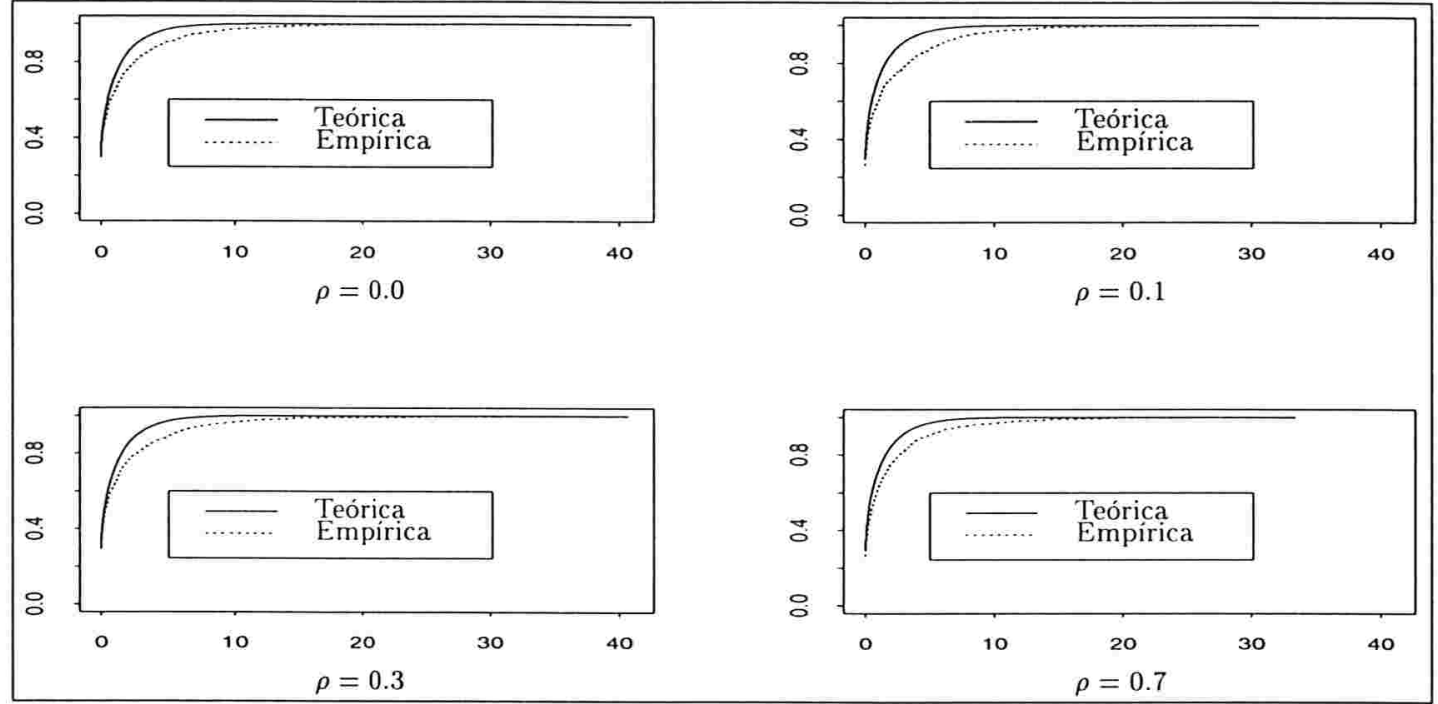

Figura 4.6.2: Poisson com regressores $n=50$ : distribuições empírica e teórica da estatística $\xi_{k p}$ com estrutura de correlação AR(1).
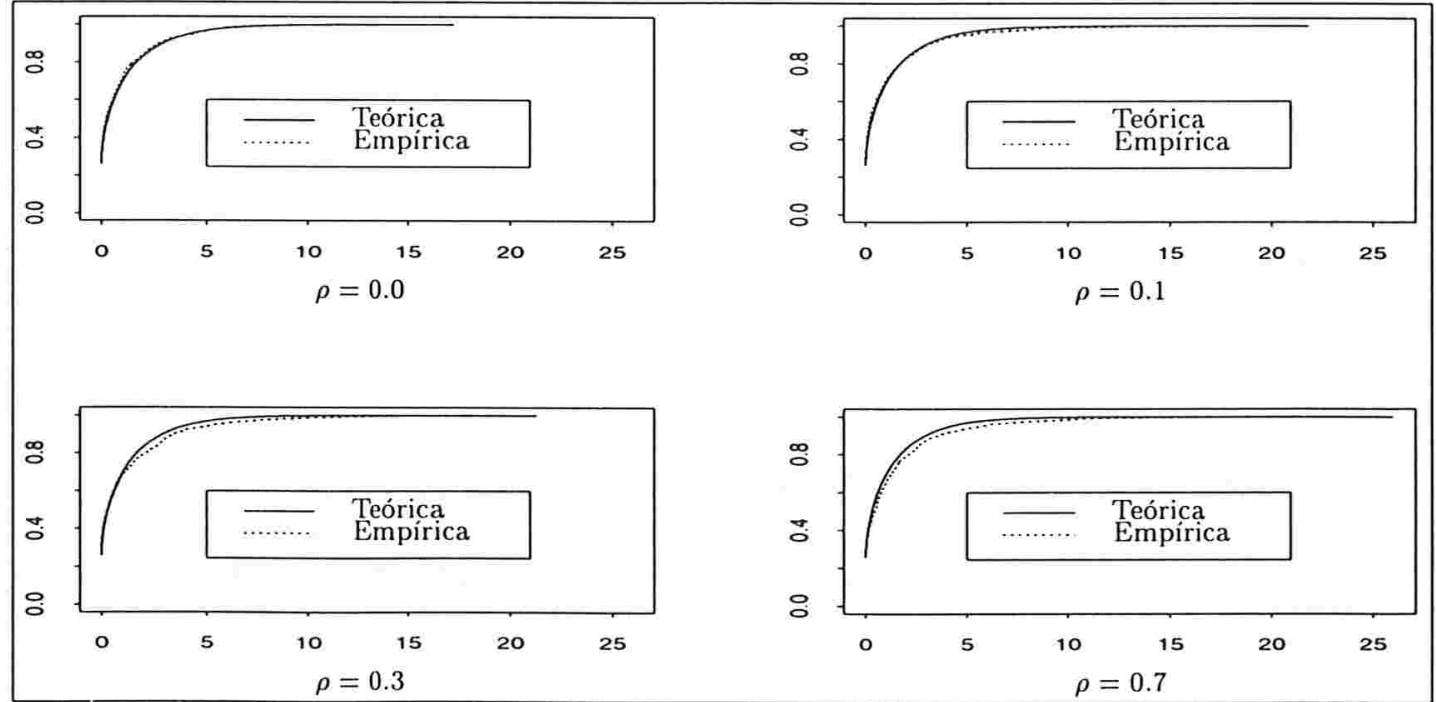
Figura 4.6.3: Poisson com regressores $n=100$ : distribuições empírica e teórica da estatística $\xi_{k p}$ com estrutura de correlação $\operatorname{AR}(1)$.

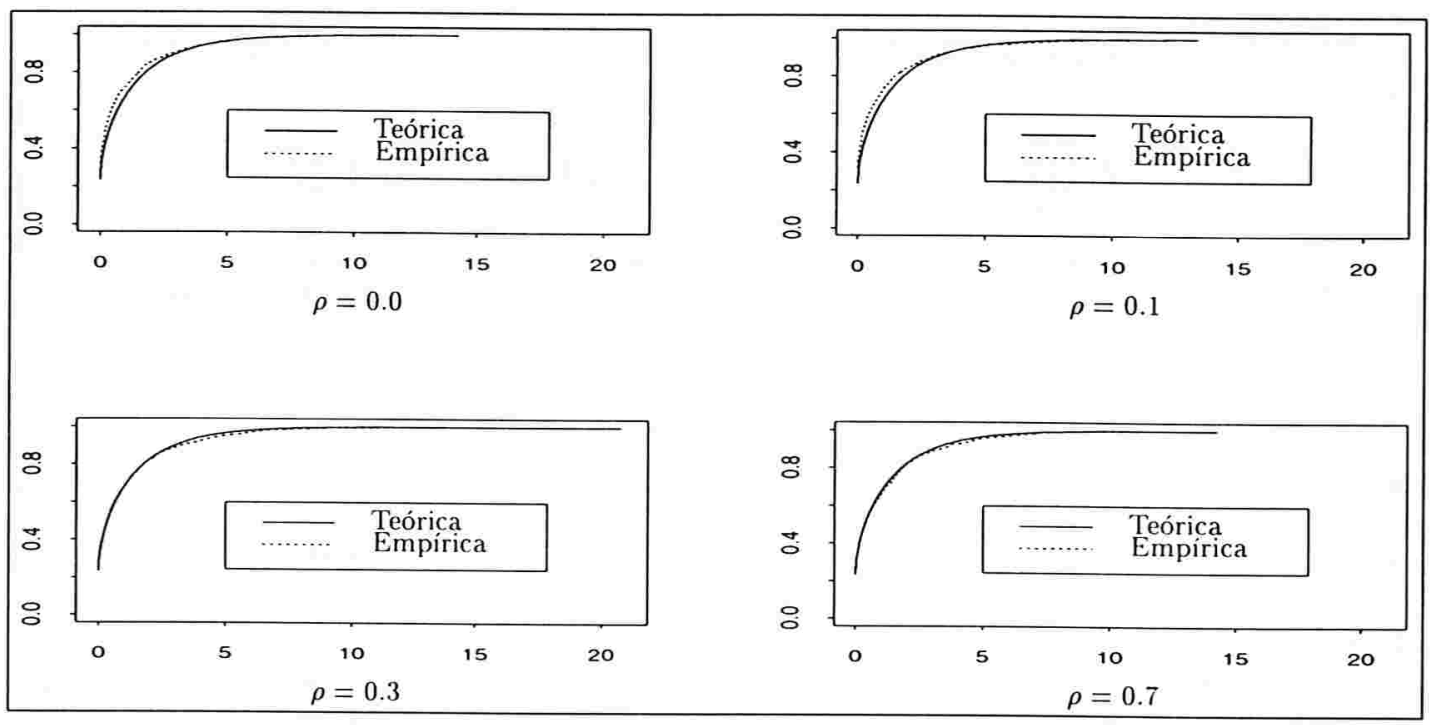

As Tabelas $4.6 .2,4.6 .3$ e 4.6 .4 a seguir apresentam o poder estimado dos testes $\xi_{k p}$ e $\xi_{w}$ para as estruturas de correlação simétrica e $\operatorname{AR}(1)$ e amostras de tamanhos 20, 50 e 100 , respectivamente. Para as duas estruturas de correlação nota-se para $n=20$ que os tamanhos empíricos dos testes $\xi_{k p}$ e $\xi_{w}$ são superiores ao tamanho nominal. Para $n=$ 50 e $n=100$ os tamanhos empíricos ficam mais próximos do valor nominal. Em geral, a diferença entre o tamanho empírico e o tamanho nominal dos testes aumenta com o aumento da correlação entre as observações. Nota-se também que o poder dos testes diminui com o aumento da correlação entre as observações. Como no caso binomial, isso é explicado pelo fato de as observações $y_{i j}(j=1,2,3,4)$ não conterem muita informação se forem altamente correlacionadas (vide Apêndice C). É evidente a superioridade da estatística de Kodde e Palm sobre a estatística de Wald, particularmente para $n=50$ e $n=100$. 
Tabela 4.6.2: Poisson com regressores: poder das estatísticas $\xi_{k p}$ e $\xi_{w}$.

\begin{tabular}{|c|c|c|c|c|c|c|c|c|}
\hline \multicolumn{3}{|c|}{$n=20$} & \multicolumn{2}{|c|}{$\alpha=0,01$} & \multicolumn{2}{|c|}{$\alpha=0.05$} & \multicolumn{2}{|c|}{$\alpha=0,10$} \\
\hline$\rho$ & $\delta_{1}$ & $\delta_{2}$ & $\xi_{k p}$ & $\xi_{w}$ & $\xi_{k p}$ & $\xi_{w}$ & $\xi_{k p}$ & $\xi_{w}$ \\
\hline \multicolumn{9}{|c|}{ Correlação simétrica } \\
\hline \multirow[t]{4}{*}{0,0} & 0,0 & 0,0 & 0,056 & 0,082 & 0,119 & 0,166 & 0,184 & 0,234 \\
\hline & 0,0 & 0,2 & 0,208 & 0,185 & 0,349 & 0,291 & 0,475 & 0,362 \\
\hline & 0,2 & 0,0 & 0,424 & 0,339 & 0,606 & 0,527 & 0,703 & 0,629 \\
\hline & 0,2 & 0,2 & 0,663 & 0,553 & 0,838 & 0,735 & 0,903 & 0,810 \\
\hline \multirow[t]{4}{*}{0,1} & 0,0 & 0,0 & 0,060 & 0,097 & 0,138 & 0,165 & 0,209 & 0,251 \\
\hline & 0,0 & 0,2 & 0,221 & 0,202 & 0,362 & 0,315 & 0,481 & 0,390 \\
\hline & 0,2 & 0,0 & 0,387 & 0,313 & 0,578 & 0,477 & 0,693 & 0,582 \\
\hline & 0,2 & 0,2 & 0,644 & 0,546 & 0,840 & 0,725 & 0,916 & 0,818 \\
\hline \multirow[t]{4}{*}{0,3} & 0,0 & 0,0 & 0,043 & 0,086 & 0,103 & 0,184 & 0,166 & 0,256 \\
\hline & 0,0 & 0,2 & 0,238 & 0,188 & 0,393 & 0,321 & 0,494 & 0,415 \\
\hline & 0,2 & 0,0 & 0,370 & 0,318 & 0,567 & 0,484 & 0,693 & 0,579 \\
\hline & 0,2 & 0,2 & 0,634 & 0,532 & 0,822 & 0,710 & 0,892 & 0,791 \\
\hline \multirow{4}{*}{0,7} & 0,0 & 0,0 & 0,046 & 0,094 & 0,103 & 0,185 & 0,168 & 0,250 \\
\hline & 0,0 & 0,2 & 0,216 & 0,183 & 0,370 & 0,310 & 0,479 & 0,396 \\
\hline & 0,2 & 0,0 & 0,372 & 0,307 & 0,571 & 0,475 & 0,675 & 0,594 \\
\hline & 0,2 & 0,2 & 0,617 & 0,532 & 0,801 & 0,689 & 0,874 & 0,770 \\
\hline \multicolumn{9}{|c|}{ Correlação AR(1) } \\
\hline \multirow[t]{4}{*}{0,0} & 0,0 & 0,0 & 0,055 & 0,089 & 0,126 & 0,167 & 0,185 & 0,238 \\
\hline & 0,0 & 0,2 & 0,203 & 0,184 & 0,354 & 0,293 & 0,471 & 0,364 \\
\hline & 0,2 & 0,0 & 0,421 & 0,346 & 0,611 & 0,533 & 0,703 & 0,623 \\
\hline & 0,2 & 0,2 & 0,672 & 0,553 & 0,840 & 0,734 & 0,903 & 0,816 \\
\hline \multirow[t]{4}{*}{0,1} & 0,0 & 0,0 & 0,068 & 0,106 & 0,159 & 0,203 & 0,235 & 0,274 \\
\hline & 0,0 & 0,2 & 0,203 & 0,183 & 0,359 & 0,304 & 0,457 & 0,387 \\
\hline & 0,2 & 0,0 & 0,360 & 0,305 & 0,572 & 0,467 & 0,688 & 0,574 \\
\hline & 0,2 & 0,2 & 0,576 & 0,468 & 0,781 & 0,650 & 0,860 & 0,744 \\
\hline \multirow[t]{4}{*}{0,3} & 0,0 & 0,0 & 0,063 & 0,093 & 0,139 & 0,177 & 0,197 & 0,255 \\
\hline & 0,0 & 0,2 & 0,183 & 0,165 & 0,338 & 0,298 & 0,454 & 0,383 \\
\hline & 0,2 & 0,0 & 0,306 & 0,262 & 0,481 & 0,418 & 0,584 & 0,508 \\
\hline & 0,2 & 0,2 & 0,495 & 0,388 & 0,702 & 0,576 & 0,798 & 0,665 \\
\hline \multirow[t]{4}{*}{0,7} & 0,0 & 0,0 & 0,054 & 0,078 & 0,118 & 0,168 & 0,191 & 0,245 \\
\hline & 0,0 & 0,2 & 0,140 & 0,118 & 0,268 & 0,225 & 0,352 & 0,308 \\
\hline & 0,2 & 0,0 & 0,201 & 0,168 & 0,380 & 0,306 & 0,492 & 0,397 \\
\hline & 0,2 & 0,2 & 0,348 & 0,268 & 0,551 & 0,438 & 0,665 & 0,528 \\
\hline
\end{tabular}


Tabela 4.6.3: Poisson com regressores: poder das estatísticas $\xi_{k p}$ e $\xi_{w}$.

\begin{tabular}{|c|c|c|c|c|c|c|c|c|}
\hline \multicolumn{3}{|c|}{$n=50$} & \multicolumn{2}{|c|}{$\alpha=0,01$} & \multicolumn{2}{|c|}{$\alpha=0,05$} & \multicolumn{2}{|c|}{$\alpha=0,10$} \\
\hline$\rho$ & $\delta_{1}$ & $\delta_{2}$ & $\xi_{k p}$ & $\xi_{w}$ & $\xi_{k p}$ & $\xi_{w}$ & $\xi_{k p}$ & $\xi_{w}$ \\
\hline \multicolumn{9}{|c|}{ Correlação simétrica } \\
\hline \multirow[t]{4}{*}{0,0} & 0,0 & 0,0 & 0,008 & 0,026 & 0,056 & 0,079 & 0,088 & 0,143 \\
\hline & 0,0 & 0,2 & 0,365 & 0,308 & 0,611 & 0,510 & 0,711 & 0,628 \\
\hline & 0,2 & 0,0 & 0,374 & 0,302 & 0,616 & 0,515 & 0,724 & 0,627 \\
\hline & 0,2 & 0,2 & 0,842 & 0,745 & 0,947 & 0,893 & 0,976 & 0,935 \\
\hline \multirow[t]{4}{*}{0,1} & 0,0 & 0,0 & 0,022 & 0,030 & 0,062 & 0,098 & 0,125 & 0,164 \\
\hline & 0,0 & 0,2 & 0,396 & 0,330 & 0,606 & 0,524 & 0,731 & 0,618 \\
\hline & 0,2 & 0,0 & 0,380 & 0,300 & 0,628 & 0,529 & 0,746 & 0,651 \\
\hline & 0.2 & 0,2 & 0,810 & 0,719 & 0,943 & 0,875 & 0,971 & 0,923 \\
\hline \multirow[t]{4}{*}{0,3} & 0,0 & 0,0 & 0,023 & 0,031 & 0,073 & 0,088 & 0,126 & 0,163 \\
\hline & 0,0 & 0,2 & 0,393 & 0,315 & 0,613 & 0,500 & 0,734 & 0,619 \\
\hline & 0,2 & 0,0 & 0,403 & 0,314 & 0,619 & 0,527 & 0,732 & 0,633 \\
\hline & 0,2 & 0,2 & 0,818 & 0,709 & 0,953 & 0,886 & 0,977 & 0,937 \\
\hline \multirow[t]{4}{*}{0,7} & 0,0 & 0,0 & 0,023 & 0,039 & 0,065 & 0,100 & 0,118 & 0,148 \\
\hline & 0,0 & 0,2 & 0,383 & 0,328 & 0,605 & 0,528 & 0,723 & 0,613 \\
\hline & 0,2 & 0,0 & 0,412 & 0,332 & 0,633 & 0,544 & 0,752 & 0,657 \\
\hline & 0,2 & 0,2 & 0,819 & 0,713 & 0,941 & 0,881 & 0,979 & 0,932 \\
\hline \multicolumn{9}{|c|}{ Correlação AR(1) } \\
\hline \multirow[t]{4}{*}{0,0} & 0,0 & 0,0 & 0,009 & 0,026 & 0,056 & 0,076 & 0,091 & 0,145 \\
\hline & 0,0 & 0,2 & 0,364 & 0,304 & 0,608 & 0,514 & 0,714 & 0,630 \\
\hline & 0,2 & 0,0 & 0,377 & 0,308 & 0,616 & 0,520 & 0,727 & 0,625 \\
\hline & 0,2 & 0,2 & 0,842 & 0,746 & 0,945 & 0,891 & 0,975 & 0,938 \\
\hline \multirow[t]{4}{*}{0,1} & 0,0 & 0,0 & 0,026 & 0,035 & 0,060 & 0,117 & 0,108 & 0,174 \\
\hline & 0,0 & 0,2 & 0,337 & 0,266 & 0,554 & 0,466 & 0,684 & 0,571 \\
\hline & 0,2 & 0,0 & 0,335 & 0,284 & 0,569 & 0,491 & 0,689 & 0,603 \\
\hline & 0,2 & 0,2 & 0,764 & 0,658 & 0,899 & 0,826 & 0,943 & 0,885 \\
\hline \multirow[t]{4}{*}{0,3} & 0,0 & 0,0 & 0,031 & 0,039 & 0,074 & 0,103 & 0,135 & 0,170 \\
\hline & 0,0 & 0,2 & 0,289 & 0,228 & 0,496 & 0,393 & 0,620 & 0,496 \\
\hline & 0,2 & 0,0 & 0,268 & 0,216 & 0,481 & 0,386 & 0,615 & 0,499 \\
\hline & 0,2 & 0,2 & 0,648 & 0,532 & 0,828 & 0,732 & 0,916 & 0,804 \\
\hline \multirow[t]{4}{*}{0,7} & 0,0 & 0,0 & 0,032 & 0,038 & 0,081 & 0,098 & 0,129 & 0,167 \\
\hline & 0,0 & 0,2 & 0,195 & 0,149 & 0,355 & 0,293 & 0,484 & 0,384 \\
\hline & 0,2 & 0,0 & 0,169 & 0,145 & 0,364 & 0,277 & 0,492 & 0,388 \\
\hline & 0,2 & 0,2 & 0,432 & 0,310 & 0,654 & 0,516 & 0,763 & 0,612 \\
\hline
\end{tabular}


Tabela 4.6.4: Poisson com regressores: poder das estatísticas $\xi_{k p}$ e $\xi_{w}$.

\begin{tabular}{|c|c|c|c|c|c|c|c|c|}
\hline \multicolumn{3}{|c|}{$n=100$} & \multicolumn{2}{|c|}{$\alpha=0,01$} & \multicolumn{2}{|c|}{$\alpha=0,05$} & \multicolumn{2}{|c|}{$\alpha=0,10$} \\
\hline$\rho$ & $\delta_{1}$ & $\delta_{2}$ & $\xi_{k p}$ & $\xi_{w}$ & $\xi_{k p}$ & $\xi_{w}$ & $\xi_{k p}$ & $\xi_{w}$ \\
\hline \multicolumn{9}{|c|}{ Correlação simétrica } \\
\hline \multirow[t]{4}{*}{0,0} & 0,0 & 0,0 & 0,009 & 0,017 & 0,049 & 0,072 & 0,089 & 0,123 \\
\hline & 0,0 & 0,2 & 0,612 & 0,536 & 0,816 & 0,756 & 0,884 & 0,849 \\
\hline & 0,2 & 0,0 & 0,678 & 0,586 & 0,839 & 0,778 & 0,912 & 0,852 \\
\hline & 0,2 & 0,2 & 0,930 & 0,872 & 0,980 & 0,960 & 0,995 & 0,978 \\
\hline \multirow{4}{*}{0,1} & 0,0 & 0,0 & 0,011 & 0,025 & 0,045 & 0,071 & 0,095 & 0,119 \\
\hline & 0,0 & 0,2 & 0,584 & 0,514 & 0,796 & 0,727 & 0,881 & 0,816 \\
\hline & 0,2 & 0,0 & 0,661 & 0,604 & 0,850 & 0,786 & 0,900 & 0,858 \\
\hline & 0,2 & 0,2 & 0,939 & 0,888 & 0,985 & 0,959 & 0,997 & 0,981 \\
\hline \multirow{4}{*}{0,3} & 0,0 & 0,0 & 0,016 & 0,027 & 0,059 & 0,086 & 0,107 & 0,148 \\
\hline & 0,0 & 0,2 & 0,552 & 0,489 & 0,777 & 0,696 & 0,859 & 0,788 \\
\hline & 0,2 & 0,0 & 0,688 & 0,588 & 0,859 & 0,798 & 0,918 & 0,878 \\
\hline & 0,2 & 0,2 & 0,936 & 0,875 & 0,986 & 0,963 & 0,994 & 0,983 \\
\hline \multirow[t]{4}{*}{0,7} & 0,0 & 0,0 & 0,019 & 0,032 & 0,073 & 0,099 & 0,107 & 0,160 \\
\hline & 0,0 & 0,2 & 0,551 & 0,467 & 0,771 & 0,692 & 0,860 & 0,794 \\
\hline & 0,2 & 0,0 & 0,695 & 0,619 & 0,865 & 0,796 & 0,927 & 0,876 \\
\hline & 0,2 & 0,2 & 0,925 & 0,857 & 0,980 & 0,960 & 0,996 & 0,977 \\
\hline \multicolumn{9}{|c|}{ Correlação AR(1) } \\
\hline \multirow[t]{4}{*}{0,0} & 0,0 & 0,0 & 0,009 & 0,017 & 0,047 & 0,072 & 0,091 & 0,127 \\
\hline & 0,0 & 0,2 & 0,613 & 0,538 & 0,814 & 0,757 & 0,884 & 0,848 \\
\hline & 0,2 & 0,0 & 0,679 & 0,585 & 0,840 & 0,775 & 0,912 & 0,852 \\
\hline & 0,2 & 0,2 & 0,930 & 0,875 & 0,981 & 0,960 & 0,995 & 0,975 \\
\hline \multirow[t]{4}{*}{0,1} & 0,0 & 0,0 & 0,081 & 0,021 & 0,052 & 0,078 & 0,089 & 0,132 \\
\hline & 0,0 & 0,2 & 0,544 & 0,461 & 0,777 & 0,686 & 0,861 & 0,794 \\
\hline & 0,2 & 0,0 & 0,583 & 0,496 & 0,796 & 0,729 & 0,875 & 0,818 \\
\hline & 0,2 & 0,2 & 0,898 & 0,835 & 0,965 & 0,938 & 0,985 & 0,960 \\
\hline \multirow[t]{4}{*}{0,3} & 0,0 & 0,0 & 0,015 & 0,024 & 0,068 & 0,084 & 0,113 & 0,142 \\
\hline & 0,0 & 0,2 & 0,367 & 0,304 & 0,619 & 0,520 & 0,739 & 0,640 \\
\hline & 0,2 & 0,0 & 0,474 & 0,388 & 0,704 & 0,605 & 0,819 & 0,713 \\
\hline & 0,2 & 0,2 & 0,805 & 0,696 & 0,933 & 0,875 & 0,966 & 0,923 \\
\hline \multirow[t]{4}{*}{0,7} & 0,0 & 0,0 & 0,011 & 0,018 & 0,068 & 0,064 & 0,112 & 0,113 \\
\hline & 0,0 & 0,2 & 0,242 & 0,187 & 0,470 & 0,347 & 0,598 & 0,473 \\
\hline & 0,2 & 0,0 & 0,302 & 0,228 & 0,534 & 0,445 & 0,681 & 0,571 \\
\hline & 0,2 & 0,2 & 0,569 & 0,462 & 0,785 & 0,671 & 0,874 & 0,770 \\
\hline
\end{tabular}




\subsection{Gama com regressores}

Amostras independentes de $\boldsymbol{y}_{i}=\left(y_{i 1}, y_{i 2}, y_{i 3}, y_{i 4}\right)^{T} \operatorname{com} y_{i j} \sim \operatorname{Gama}\left(\mu_{i j}, \phi\right), j=1,2,3,4$, $i=1,2, \ldots, n$, foram geradas com $\phi=3$ e estruturas de correlação simétrica e AR(1) para as observações da mesma unidade experimental.

Usando o modelo de regressão linear

$$
\mu_{i j}^{-1}=\beta_{0}+\beta_{1} x_{1 i}+\beta_{2} x_{2 i}
$$

com $\beta_{0}=1$, simulações foram conduzidas para verificar o poder da estatística de Kodde e Palm para testar as seguintes hipóteses:

$$
H_{0}: \beta_{1}=1, \beta_{2}=1 \text { versus } H_{1}: \beta_{1} \geq 1, \beta_{2} \geq 1
$$

com pelo menos uma desigualdade estrita em $H_{1}$. Na hipótese alternativa foram considerados $\beta_{1}=1+\delta_{1}$ e $\beta_{2}=1+\delta_{2}$ para valores de $\left(\delta_{1}, \delta_{2}\right)=(0 ; 0),(0 ; 0,5),(0,5 ; 0)$ e $(0,5 ; 0,5)$ e valores de $\rho=0,0 ; 0,1 ; 0,3$ e 0,7 . As hipóteses acima são equivalentes às hipóteses (4.5.1) com

$$
\boldsymbol{\beta}=\left(\begin{array}{c}
\beta_{0} \\
\beta_{1} \\
\beta_{2}
\end{array}\right), \quad \boldsymbol{R}=\left[\begin{array}{lll}
0 & 1 & 0 \\
0 & 0 & 1
\end{array}\right] \text { e } \boldsymbol{r}=\left(\begin{array}{l}
1 \\
1
\end{array}\right) .
$$

Os resultados da simulação indicam que a diferença entre as distribuições teórica e empirica, para as duas estruturas de correlação, simétrica e autoregressiva, é significativa para amostras de tamanho $n=20$ e não é significativa para amostras de tamanho $n=100$. Para amostras de tamanho $n=50$ e correlação simétrica, a aproximação entre essas distribuições é razoável somente para $\rho=0,7$ e para correlação $\operatorname{AR}(1)$ a aproximação foi razoável para $\rho=0,1$ e $\rho=0,7$, conforme os resultados decorrrentes 
da aplicação do teste de Kolmogorov-Smirnov, os quais são apresentados a seguir na Tabela 4.7.1.

Tabela 4.7.1: Gama com regressores: KolmogorovSmirnov (P-ralor).

\begin{tabular}{cccc}
\hline \multicolumn{4}{c}{ Tamanho da amostra } \\
\hline$\rho$ & $n=20$ & $n=50$ & $n=100$ \\
\hline \hline \multicolumn{4}{c}{ Correlação simétrica } \\
\hline 0,0 & $0,1213(0,0000)$ & $0,0592(0,0240)$ & $0,0378(0,3264)$ \\
0,1 & $0,0855(0,0002)$ & $0,0561(0,0375)$ & $0,0343(0,4469)$ \\
0,3 & $0,0859(0,0002)$ & $0,0640(0,0113)$ & $0,0320(0,5387)$ \\
0,7 & $0,1012(0,0000)$ & $0,0522(0,0641)$ & $0,0220(0,9200)$ \\
\hline \multicolumn{5}{c}{ Correlação AR(1) } \\
\hline \hline 0,0 & $0,1170(0,0000)$ & $0,0610(0,0181)$ & $0,0398(0,2702)$ \\
0,1 & $0,1251(0,0000)$ & $0,0320(0,5381)$ & $0,0218(0,9237)$ \\
0,3 & $0,1222(0,0000)$ & $0,0341(0,4552)$ & $0,0223(0,9118)$ \\
0,7 & $0,1143(0,0000)$ & $0,0301(0,6184)$ & $0,0275(0,7277)$ \\
\hline
\end{tabular}

As Figuras 4.7.1, 4.7.2 e 4.7.3 a seguir mostram, para a estatística $\xi_{k p}$, a distribuição empírica e a distribuição teórica dada em (4.2.1) para a estrutura de correlação autoregressiva, com todos os valores de $\rho$ considerados no estudo e amostras de tamanho 20, 50 e 100 , respectivamente. 
Figura 4.7.1: Gama com regressores $n=20$ : distribuições empírica e teórica da estatística $\xi_{k p}$ com estrutura de correlação $\operatorname{AR}(1)$.
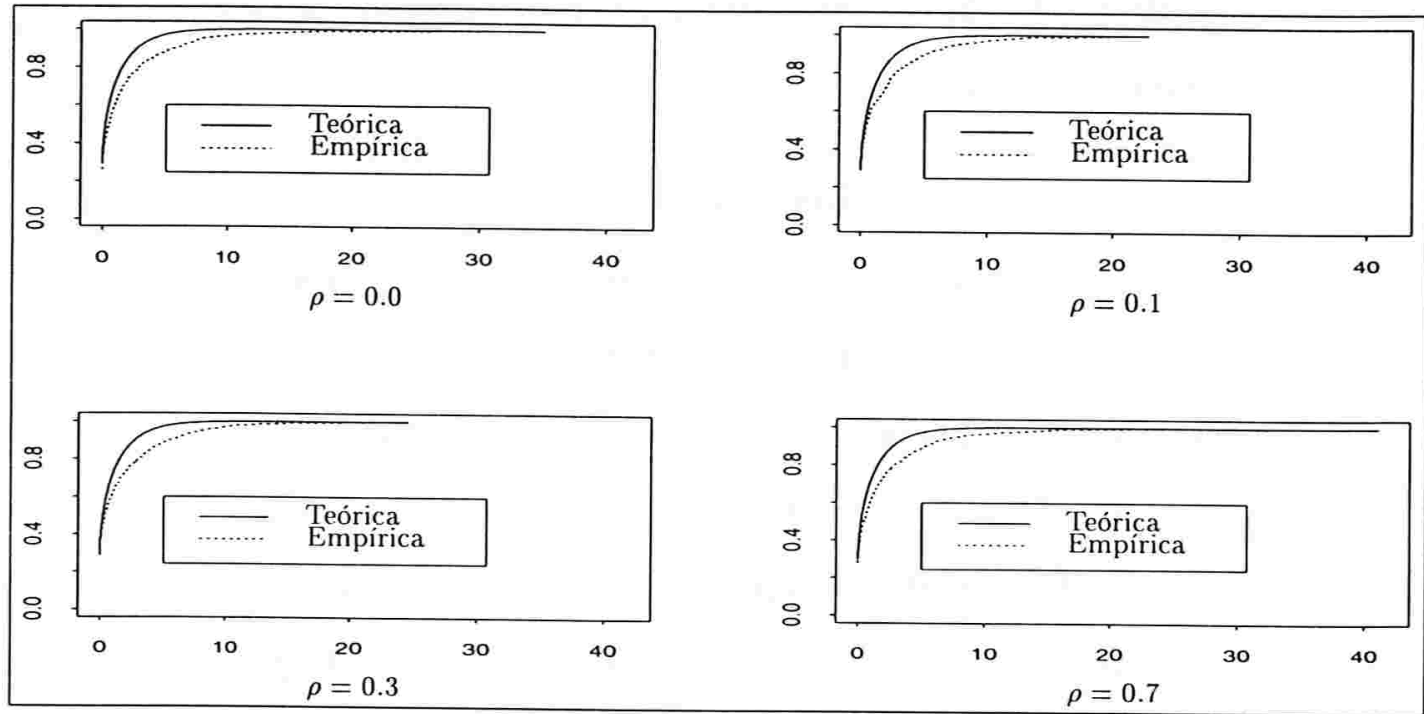

Figura 4.7.2: Gama com regressores $n=50$ : distribuições empírica e teórica da estatística $\xi_{k p}$ com estrutura de correlação $\operatorname{AR}(1)$.
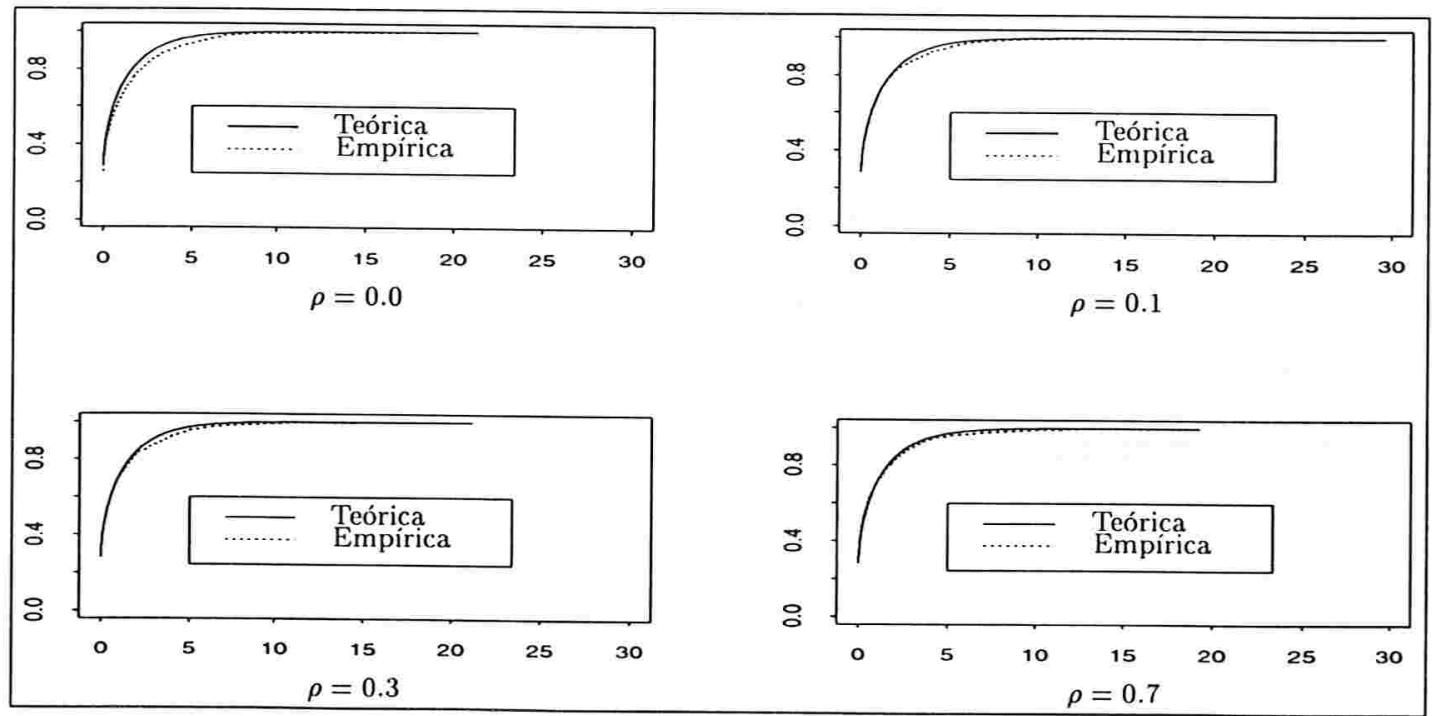
Figura 4.7.3: Gama com regressores $n=100$ : distribuições empírica e teórica da estatística $\xi_{k p}$ com estrutura de correlação $\mathrm{AR}(1)$.

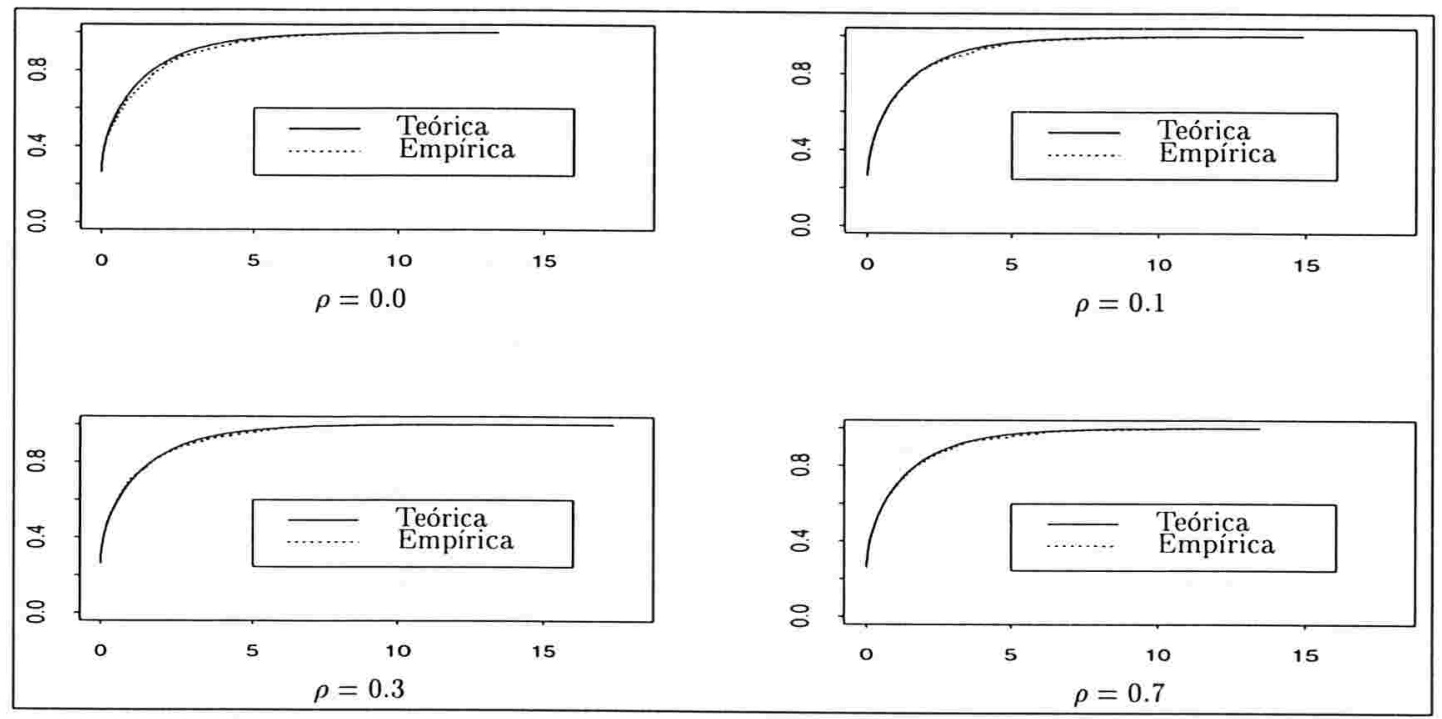

As Tabelas 4.7.2, 4.7.3 e 4.7.4 a seguir apresentam o poder estimado dos testes $\xi_{k p}$ e $\xi_{w}$ para as estruturas de correlação simétrica e autoregressiva e amostras de tamanhos 20 , 50 e 100, respectivamente. Para as duas estruturas de correlação, simétrica e AR(1), nota-se para $n=20$ que os tamanhos empíricos dos testes $\xi_{k p}$ e $\xi_{w}$ são superiores ao tamanho nominal. Para $n=50$ e $n=100$ os tamanhos empíricos ficam mais próximos do valor nominal. Em geral, a diferença entre o tamanho empírico e o tamanho nominal dos testes aumenta com o aumento da correlação entre as observações. Nota-se também que, neste caso de distribuição gama, que a do poder dos testes com o aumento da correlação entre as observações não é muito clara como nas outras distribuições consideradas. É evidente a superioridade da estatística de Kodde e Palm sobre a estatística de Wald, para todos os tamanhos de amostras. 
Tabela 4.7.2: Gama com regressores: poder das estatísticas $\xi_{k p}$ e $\xi_{w}$.

\begin{tabular}{|c|c|c|c|c|c|c|c|c|}
\hline \multicolumn{3}{|c|}{$n=20$} & \multicolumn{2}{|c|}{$\alpha=0,01$} & \multicolumn{2}{|c|}{$\alpha=0,05$} & \multicolumn{2}{|c|}{$\alpha=0.10$} \\
\hline$\rho$ & $\delta_{1}$ & $\delta_{2}$ & $\xi_{k p}$ & $\xi_{w}$ & $\xi_{k p}$ & $\xi_{w}$ & $\xi_{k p}$ & $\xi_{w}$ \\
\hline \multicolumn{9}{|c|}{ Correlação simétrica } \\
\hline \multirow[t]{4}{*}{0,0} & 0,0 & 0,0 & 0,068 & 0,101 & 0,142 & 0,193 & 0,215 & 0.262 \\
\hline & 0,0 & 0,5 & 0,151 & 0,151 & 0,292 & 0,251 & 0,386 & 0,338 \\
\hline & 0,5 & 0,0 & 0,225 & 0,203 & 0,396 & 0,340 & 0,512 & 0,432 \\
\hline & 0,5 & 0,5 & 0,310 & 0,245 & 0,512 & 0,396 & 0,631 & 0.495 \\
\hline \multirow[t]{4}{*}{0,1} & 0,0 & 0,0 & 0,052 & 0,079 & 0,126 & 0,180 & 0,174 & 0.249 \\
\hline & 0,0 & 0,5 & 0,127 & 0,128 & 0,261 & 0,227 & 0,377 & 0,321 \\
\hline & 0,5 & 0,0 & 0.213 & 0,137 & 0,388 & 0,330 & 0,508 & 0.413 \\
\hline & 0,5 & 0.5 & 0.298 & 0,226 & 0,524 & 0,379 & 0,633 & 0.511 \\
\hline \multirow[t]{4}{*}{0,3} & 0,0 & 0,0 & 0,051 & 0,087 & 0,114 & 0,181 & 0,179 & 0,248 \\
\hline & 0,0 & 0,5 & 0,158 & 0,143 & 0,308 & 0,260 & 0,411 & 0,352 \\
\hline & 0,5 & 0,0 & 0,267 & 0,229 & 0,464 & 0,384 & 0,586 & 0,489 \\
\hline & 0,5 & 0,5 & 0.396 & 0,292 & 0,611 & 0,464 & 0,727 & 0,589 \\
\hline \multirow[t]{4}{*}{0,7} & 0,0 & 0,0 & 0,064 & 0,102 & 0,139 & 0,205 & 0,198 & 0,280 \\
\hline & 0,0 & 0,5 & 0,198 & 0,174 & 0,320 & 0,290 & 0,411 & 0,364 \\
\hline & 0,5 & 0,0 & 0,298 & 0,256 & 0,471 & 0,416 & 0,584 & 0,507 \\
\hline & 0,5 & 0,5 & 0,388 & 0,310 & 0,606 & 0,487 & 0,719 & 0,580 \\
\hline \multicolumn{9}{|c|}{ Correlação AR(1) } \\
\hline \multirow[t]{4}{*}{0,0} & 0,0 & 0,0 & 0,071 & 0,101 & 0,146 & 0,198 & 0,210 & 0,262 \\
\hline & 0,0 & 0,5 & 0,149 & 0,147 & 0,293 & 0,258 & 0,388 & 0,341 \\
\hline & 0,5 & 0,0 & 0,233 & 0,202 & 0,404 & 0,342 & 0,512 & 0,429 \\
\hline & 0,5 & 0,5 & 0,311 & 0,246 & 0,508 & 0,396 & 0,630 & 0,493 \\
\hline \multirow[t]{4}{*}{0,1} & 0,0 & 0,0 & 0,057 & 0,096 & 0,140 & 0,190 & 0,194 & 0,253 \\
\hline & 0,0 & 0,5 & 0,168 & 0,154 & 0,301 & 0,271 & 0,383 & 0,340 \\
\hline & 0,5 & 0,0 & 0,254 & 0,216 & 0,434 & 0,357 & 0,530 & 0,458 \\
\hline & 0,5 & 0,5 & 0,347 & 0,268 & 0,536 & 0,418 & 0,660 & 0,517 \\
\hline \multirow[t]{4}{*}{0,3} & 0,0 & 0,0 & 0,068 & 0,105 & 0,152 & 0,200 & 0,222 & 0,269 \\
\hline & 0,0 & 0,5 & 0,227 & 0,191 & 0,364 & 0,316 & 0,451 & 0,397 \\
\hline & 0,5 & 0,0 & 0,329 & 0,292 & 0,505 & 0,432 & 0,629 & 0,525 \\
\hline & 0,5 & 0,5 & 0,465 & 0,365 & 0,652 & 0,545 & 0,744 & 0,616 \\
\hline \multirow[t]{4}{*}{0,7} & 0,0 & 0,0 & 0,061 & 0,103 & 0,148 & 0,199 & 0,210 & 0,273 \\
\hline & 0,0 & 0,5 & 0,170 & 0,172 & 0,328 & 0,288 & 0,423 & 0,382 \\
\hline & 0,5 & 0,0 & 0,262 & 0,222 & 0,427 & 0,365 & 0,550 & 0,454 \\
\hline & 0,5 & 0,5 & 0,368 & 0,290 & 0,560 & 0,447 & 0,672 & 0,546 \\
\hline
\end{tabular}


Tabela 4.7.3: Gama com regressores: poder das estatísticas $\xi_{k p}$ e $\xi_{w}$.

\begin{tabular}{|c|c|c|c|c|c|c|c|c|}
\hline \multicolumn{3}{|c|}{$n=50$} & \multicolumn{2}{|c|}{$\alpha=0.01$} & \multicolumn{2}{|c|}{$\alpha=0,05$} & \multicolumn{2}{|c|}{$\alpha=0,10$} \\
\hline$\rho$ & $\delta_{1}$ & $\delta_{2}$ & $\xi_{k p}$ & $\xi_{w}$ & $\xi_{k p}$ & $\xi_{w}$ & $\xi_{k p}$ & $\xi_{w}$ \\
\hline \multicolumn{9}{|c|}{ Correlação simétrica } \\
\hline \multirow[t]{4}{*}{0,0} & 0,0 & 0,0 & 0,021 & 0,042 & 0,088 & 0,102 & 0,142 & 0,159 \\
\hline & 0,0 & 0,5 & 0,220 & 0,170 & 0,439 & 0,343 & 0,552 & 0,441 \\
\hline & 0,5 & 0,0 & 0,304 & 0,228 & 0,526 & 0,413 & 0,645 & 0,523 \\
\hline & 0,5 & 0,5 & 0,543 & 0,417 & 0,755 & 0,620 & 0,824 & 0,720 \\
\hline \multirow[t]{4}{*}{0,1} & 0,0 & 0,0 & 0,013 & 0,023 & 0,078 & 0,083 & 0,148 & 0,157 \\
\hline & 0,0 & 0,5 & 0,259 & 0,199 & 0,491 & 0,380 & 0,638 & 0,506 \\
\hline & 0,5 & 0,0 & 0,334 & 0,259 & 0,551 & 0,458 & 0,662 & 0,546 \\
\hline & 0,5 & 0,5 & 0,598 & 0,490 & 0,803 & 0,679 & 0,870 & 0,769 \\
\hline \multirow[t]{4}{*}{0,3} & 0,0 & 0,0 & 0,020 & 0,030 & 0,071 & 0,094 & 0,131 & 0,155 \\
\hline & 0,0 & 0,5 & 0,374 & 0,287 & 0,601 & 0,491 & 0,729 & 0,609 \\
\hline & 0,5 & 0,0 & 0,451 & 0,374 & 0,672 & 0,560 & 0,776 & 0,672 \\
\hline & 0,5 & 0,5 & 0,742 & 0,642 & 0,889 & 0,804 & 0,938 & 0,871 \\
\hline \multirow[t]{4}{*}{0,7} & 0,0 & 0,0 & 0,023 & 0,039 & 0,074 & 0,110 & 0,136 & 0,171 \\
\hline & 0,0 & 0,5 & 0,353 & 0,290 & 0,582 & 0,475 & 0,683 & 0,577 \\
\hline & 0,5 & 0,0 & 0,465 & 0,365 & 0,665 & 0,577 & 0,775 & 0,689 \\
\hline & 0,5 & 0,5 & 0,744 & 0,631 & 0,884 & 0,793 & 0,938 & 0,861 \\
\hline \multicolumn{9}{|c|}{ Correlação AR $(1)$} \\
\hline \multirow[t]{4}{*}{0,0} & 0,0 & 0,0 & $\overline{0,022}$ & 0,041 & 0,092 & 0,100 & 0,144 & 0,159 \\
\hline & 0,0 & 0,5 & 0,222 & 0,171 & 0,435 & 0,341 & 0,554 & 0,453 \\
\hline & 0,5 & 0,0 & 0,306 & 0,235 & 0,526 & 0,418 & 0,645 & 0,520 \\
\hline & 0,5 & 0,5 & 0,544 & 0,411 & 0,754 & 0,622 & 0,827 & 0,730 \\
\hline \multirow[t]{4}{*}{0,1} & 0,0 & 0,0 & 0,019 & 0,031 & 0,074 & 0,085 & 0,126 & 0,154 \\
\hline & 0,0 & 0,5 & 0,259 & 0,201 & 0,478 & 0,368 & 0,606 & 0,481 \\
\hline & 0,5 & 0,0 & 0,332 & 0,256 & 0,561 & 0,466 & 0,669 & 0,569 \\
\hline & 0,5 & 0,5 & 0,602 & 0,477 & 0,808 & 0,684 & 0,883 & 0,786 \\
\hline \multirow[t]{4}{*}{0,3} & 0,0 & 0,0 & 0,022 & 0,031 & 0,076 & 0,092 & 0,133 & 0,150 \\
\hline & 0,0 & 0,5 & 0,387 & 0,297 & 0,605 & 0,510 & 0,710 & 0,609 \\
\hline & 0,5 & 0,0 & 0,497 & 0,405 & 0,711 & 0,619 & 0,804 & 0,724 \\
\hline & 0,5 & 0,5 & 0,784 & 0,666 & 0,920 & 0,834 & 0,958 & 0,906 \\
\hline \multirow[t]{4}{*}{0,7} & 0,0 & 0,0 & 0,026 & 0,030 & 0,062 & 0,085 & 0,117 & 0,146 \\
\hline & 0,0 & 0,5 & 0,250 & 0,197 & 0,462 & 0,380 & 0,582 & 0,483 \\
\hline & 0,5 & 0,0 & 0,337 & 0,269 & 0,567 & 0,458 & 0,679 & 0,565 \\
\hline & 0,5 & 0,5 & 0,605 & 0,467 & 0,801 & 0,685 & 0,878 & 0,771 \\
\hline
\end{tabular}


Tabela 4.7.4: Gama com regressores: poder das estatísticas $\xi_{k p}$ e $\xi_{w}$.

\begin{tabular}{|c|c|c|c|c|c|c|c|c|}
\hline \multicolumn{3}{|c|}{$n=100$} & \multicolumn{2}{|c|}{$\alpha=0,01$} & \multicolumn{2}{|c|}{$\alpha=0,05$} & \multicolumn{2}{|c|}{$\alpha=0,10$} \\
\hline$\rho$ & $\delta_{1}$ & $\delta_{2}$ & $\xi_{k p}$ & $\xi_{w}$ & $\xi_{k p}$ & $\xi_{w}$ & $\xi_{k p}$ & $\xi_{w}$ \\
\hline \multicolumn{9}{|c|}{ Correlação simétrica } \\
\hline \multirow[t]{4}{*}{0,0} & 0,0 & 0,0 & 0,014 & 0,012 & 0,067 & 0,078 & 0,119 & 0,130 \\
\hline & 0,0 & 0,5 & 0,386 & 0,319 & 0,624 & 0,522 & 0,743 & 0,642 \\
\hline & 0,5 & 0,0 & 0,438 & 0,353 & 0,673 & 0,563 & 0,780 & 0,674 \\
\hline & 0,5 & 0,5 & 0,728 & 0,618 & 0,884 & 0,789 & 0,941 & 0,861 \\
\hline \multirow[t]{4}{*}{0,1} & 0,0 & 0,0 & 0,019 & 0,014 & 0,066 & 0,068 & 0,117 & 0,114 \\
\hline & 0,0 & 0,5 & 0,480 & 0,401 & 0,701 & 0,608 & 0,807 & 0,709 \\
\hline & 0,5 & 0,0 & 0,507 & 0,413 & 0,719 & 0,621 & 0,837 & 0,723 \\
\hline & 0,5 & 0,5 & 0,808 & 0,710 & 0,933 & 0,872 & 0,975 & 0,919 \\
\hline \multirow[t]{4}{*}{0,3} & 0,0 & 0,0 & 0,019 & 0,017 & 0,069 & 0,068 & 0,118 & 0,126 \\
\hline & 0,0 & 0,5 & 0,643 & 0,565 & 0,834 & 0,755 & 0,908 & 0,837 \\
\hline & 0,5 & 0,0 & 0,666 & 0,574 & 0,860 & 0,783 & 0,921 & 0,858 \\
\hline & 0,5 & 0,5 & 0,923 & 0,866 & 0,983 & 0,956 & 0,995 & 0,978 \\
\hline \multirow[t]{4}{*}{0,7} & 0,0 & 0,0 & 0,022 & 0,024 & 0,068 & 0,083 & 0,115 & 0,129 \\
\hline & 0,0 & 0,5 & 0,644 & 0,554 & 0,844 & 0,760 & 0,908 & 0,844 \\
\hline & 0,5 & 0,0 & 0,677 & 0,588 & 0,840 & 0,779 & 0,905 & 0,843 \\
\hline & 0,5 & 0,5 & 0,927 & 0,878 & 0,974 & 0,954 & 0,990 & 0,970 \\
\hline \multicolumn{9}{|c|}{ Correlação AR(1) } \\
\hline \multirow[t]{4}{*}{0,0} & 0,0 & 0,0 & 0,015 & 0,013 & 0,067 & 0,074 & 0,119 & 0,128 \\
\hline & 0,0 & 0,5 & 0,386 & 0,318 & 0,622 & 0,523 & 0,743 & 0,643 \\
\hline & 0,5 & 0,0 & 0,437 & 0,352 & 0,673 & 0,565 & 0,781 & 0,680 \\
\hline & 0,5 & 0,5 & 0,726 & 0,619 & 0,884 & 0,783 & 0,941 & 0,859 \\
\hline \multirow[t]{4}{*}{0,1} & 0,0 & 0,0 & 0,016 & 0,017 & 0,063 & 0,063 & 0,116 & 0,121 \\
\hline & 0,0 & 0,5 & 0,509 & 0,415 & 0,740 & 0,648 & 0,836 & 0,744 \\
\hline & 0,5 & 0,0 & 0,531 & 0,445 & 0,755 & 0,655 & 0,846 & 0,758 \\
\hline & 0,5 & 0,5 & 0,829 & 0,733 & 0,955 & 0,883 & 0,982 & 0,943 \\
\hline \multirow[t]{4}{*}{0,3} & 0,0 & 0,0 & 0,011 & 0,016 & 0,066 & 0,059 & 0,113 & 0,120 \\
\hline & 0,0 & 0,5 & 0,718 & 0,616 & 0,871 & 0,807 & 0,922 & 0,873 \\
\hline & 0,5 & 0,0 & 0,724 & 0,634 & 0,894 & 0,832 & 0,943 & 0,893 \\
\hline & 0,5 & 0,5 & 0,959 & 0,912 & 0,992 & 0,975 & 0,997 & 0,990 \\
\hline \multirow[t]{4}{*}{0,7} & 0,0 & 0,0 & 0,014 & 0,019 & 0,063 & 0,082 & 0,115 & 0,137 \\
\hline & 0,0 & 0,5 & 0,504 & 0,417 & 0,737 & 0,643 & 0,830 & 0,755 \\
\hline & 0,5 & 0,0 & 0,531 & 0,443 & 0,752 & 0,656 & 0,858 & 0,756 \\
\hline & 0,5 & 0,5 & 0,842 & 0,751 & 0,949 & 0,893 & 0,979 & 0,938 \\
\hline
\end{tabular}




\section{Capítulo 5}

\section{Proposta Alternativa}

\subsection{Introdução}

Vamos discutir neste capítulo uma proposta alternativa de testes unilaterais para o caso em que $H_{0}: \boldsymbol{\mu}=0$ versus $H_{1}: \boldsymbol{\mu} \geq \mathbf{0}$, onde $\boldsymbol{\mu}=\left(\mu_{1}, \mu_{2}, \ldots, \mu_{k}\right)^{T}$ com pelo menos uma desigualdade estrita em $H_{1}$. A proposta introduzida por Follmann (1996) é baseada na estatística $T^{2}$ de Hotelling e evita o cálculo dos pesos $w(k, \ell ; \Omega)$ 's que aparecem como coeficientes da distribuição nula assintótica das estatísticas $\xi_{r v}, \xi_{s r}, \xi_{w}$ e $\xi_{k p}$, definidas no Capítulo 3. Como nosso interesse é investigar o uso de testes unilaterais através de EEGs, iremos enfocar a aplicação do teste de Follmann apenas para essa situação.

\subsection{Teste de Follmann}

Vamos considerar inicialmente o caso de $g$ grupos através de EEGs definido na Seção 3.5.1 e o problema tradicional de teste de hipóteses

$$
H_{0}: \mu=0 \text { versus } H_{1}^{*}: \mu \neq 0 \text {. }
$$


Como é bem conhecido, a forma quadrática

$$
\xi_{w}^{*}=\hat{\mu}^{T} \hat{\mathbf{V}}_{\mu}^{-1} \hat{\mu}
$$

segue, sob $H_{0}$, uma distribuição assintótica qui-quadrado central com $g$ graus de liberdade. Seguindo Follmann (1996) vamos propor a seguir um procedimento bastante simples no sentido de ganhar poder com a estatística definida em (5.2.1) para alternativas do tipo $H_{1}: \boldsymbol{\mu} \geq 0$ com pelo menos uma desigualdade estrita.

Considere, então, o procedimento que rejeita, para um nível de significância $\alpha$, valores de $\xi_{w}^{*}$ que excedam o valor crítico de nível $2 \alpha$ da distribuição $\chi_{g}^{2}$ com $\sum_{j=1}^{g} \hat{\mu}_{j}>0$. Esse procedimento será denominado $\xi_{w}^{+}$uma vez que requer que a soma dos elementos de $\hat{\boldsymbol{\mu}}$ seja positiva. É interessante notar que o procedimento proposto tem a mesma lógica do caso univariado com a estatística $\xi_{w}$ quando testamos $H_{0}: \mu=0$ versus $H_{1}: \mu>0$. A estatística $\xi_{w}$ definida na Seção 3.3, fica nesse caso dada por $\xi_{w}=\hat{\mu}^{2} / \hat{V} \operatorname{ar}(\hat{\mu})$ e rejeita valores que excedam o valor crítico de nível $2 \alpha$ da distribuição $\chi_{1}^{2}$, além de requerer que $\hat{\mu}>0$. Portanto, o procedimento proposto $\xi_{w}^{+}$pode ser interpretado como uma generalização do teste de Wald $\xi_{w}$. As principais vantagens desse novo procedimento são que o mesmo não requer o cálculo dos pesos $w(k, \ell ; \Omega)$ 's nem das estimativas $\overline{\boldsymbol{\mu}} \mathrm{e}$ $\tilde{\boldsymbol{\mu}}$; todas as quantidades são avaliadas em $\hat{\boldsymbol{\mu}}$. Resta saber o que ocorre com o poder de $\xi_{w}^{+}$quando comparado com o poder de $\xi_{k p}$, por exemplo, no caso de EEGs.

Através do teorema dado a seguir (vide Follmann, 1996) podemos obter limites estreitos para o poder do teste proposto bem como a distribuição nula assintótica de $\xi_{w}^{+}$.

Teorema 5.1. Seja $\boldsymbol{X}$ uma variável aleatória com distribuição normal k-variada de média $\boldsymbol{\mu}=\left(\mu_{1}, \mu_{2}, \ldots, \mu_{k}\right)^{T}$ e matriz de covariância $\mathbf{V}_{\boldsymbol{\mu}}$. Seja $\boldsymbol{S} \boldsymbol{S}^{T}$ a decomposição de Cholesky de $\mathrm{V}_{\boldsymbol{\mu}}$. Defina o vetor a de modo que $\boldsymbol{a}=\left(a_{1}, a_{2}, \ldots, a_{k}\right)^{T}$ tal que $a_{j}=\sum_{i=1}^{k} s_{i j}$ é a soma da j-ésima coluna de $\boldsymbol{S}$ e seja $T_{\boldsymbol{a}}(\cdot)$ uma transformação ortogonal do vetor $\boldsymbol{a}$ 
sobre 1. Então,

$$
P\left(\boldsymbol{X}^{T} \mathrm{~V}_{\boldsymbol{\mu}}^{-1} \boldsymbol{X} \geq c \bigcap \mathbf{1}^{T} \boldsymbol{X}>0\right) \geq P\left(\boldsymbol{X}^{T} \mathrm{~V}_{\boldsymbol{\mu}}^{-1} \boldsymbol{X} \geq c\right) P\left(\mathbf{1}^{T} \boldsymbol{X}>\mathbf{0}\right)
$$

sempre que $\mathbf{1}^{T} T_{a}\left(\boldsymbol{S}^{-1} \boldsymbol{\mu}\right) \geq \mathbf{0}$ com igualdade quando $\boldsymbol{\mu}=\mathbf{0}$.

Corolário 5.1. Sob as condiçôes dadas para o vetor aleatório $\boldsymbol{X}$, temos que

$$
P\left(\boldsymbol{X}^{T} \mathrm{~V}_{\boldsymbol{\mu}}^{-1} \boldsymbol{X} \geq c \bigcap \mathbf{1}^{T} \boldsymbol{X}>0\right) \leq P\left(\boldsymbol{X}^{T} \mathrm{~V}_{\boldsymbol{\mu}}^{-1} \boldsymbol{X} \geq c\right) P\left(\mathbf{1}^{T} \boldsymbol{X}>0\right)
$$

sempre que $\mathbf{1}^{T} T_{a}\left(\boldsymbol{S}^{-1} \boldsymbol{\mu}\right) \leq \mathbf{0}$.

Esse teorema apresenta limites inferiores para o poder do teste $\xi_{w}^{+}$para alternativas de interesse (quando $\boldsymbol{\mu}$ tende a ser positivo). Esses limites podem ser considerados estreitos uma vez que a igualdade de (5.2.2) ocorre quando $\mathbf{1}^{T} T_{a}\left(\boldsymbol{S}^{-1} \boldsymbol{\mu}\right)=0(\boldsymbol{\mu}=\mathbf{0})$.

Portanto, se substituirmos $\boldsymbol{X}$ por $\hat{\boldsymbol{\mu}}$ e $\mathrm{V}_{\boldsymbol{\mu}}$ por $\hat{\mathbf{V}}_{\boldsymbol{\mu}}$ e fizermos $g=k$ temos para grandes amostras e sob $H_{0}: \boldsymbol{\mu}=\mathbf{0}$ que

$$
P\left(\hat{\boldsymbol{\mu}}^{T} \hat{\mathbf{V}}_{\boldsymbol{\mu}}^{-1} \hat{\boldsymbol{\mu}} \geq c \bigcap \sum_{i=1}^{g} \hat{\mu}_{i}>0\right)=P\left(\hat{\boldsymbol{\mu}}^{T} \hat{\mathbf{V}}_{\boldsymbol{\mu}}^{-1} \hat{\boldsymbol{\mu}} \geq c\right) P\left(\sum_{i=1}^{g} \hat{\mu}_{i}>0\right) .
$$

Note que sob $H_{0}: \boldsymbol{\mu}=0$ temos que $P\left(\sum_{i=1}^{g} \hat{\mu}_{i}>0\right)=1 / 2$. Assim, para um nível de significância $\alpha$ rejeitamos $H_{0}$ se $\hat{\mu}^{T} \hat{\mathbf{V}}_{\boldsymbol{\mu}}^{-1} \hat{\mu} \geq c_{2 \alpha}$ e $\sum_{i=1}^{g} \hat{\mu}_{i}>0$, onde $c_{2 \alpha}$ é tal que

$$
\begin{aligned}
\frac{1}{2} P\left(\hat{\boldsymbol{\mu}}^{T} \hat{\mathbf{V}}_{\boldsymbol{\mu}}^{-1} \hat{\boldsymbol{\mu}} \geq c_{2 \alpha}\right) & =\alpha, \\
P\left(\xi_{w}^{*} \geq c_{2 \alpha}\right) & =2 \alpha \\
P\left(\chi_{g}^{2} \geq c_{2 \alpha}\right) & =2 \alpha,
\end{aligned}
$$

ou seja, o nível crítico $c_{2 \alpha}$ é obtido de uma distribuição qui-quadrado com $g$ graus de liberdade. Podemos generalizar facilmente o procedimento de Follmann para o caso de testar

$$
H_{0}: \boldsymbol{R} \boldsymbol{\beta}=\boldsymbol{r} \text { versus } H_{1}: \boldsymbol{R} \boldsymbol{\beta} \geq \boldsymbol{r}
$$


com pelo menos uma desigualdade estrita em $H_{1}$. Como vimos na Seção 3.5 o estimador $\hat{\boldsymbol{\beta}}_{G}$ é assintoticamente normal com média $\boldsymbol{\beta}$ e variância estimada $\hat{\mathrm{V}}_{G}$. A estatística $T^{2}$ de Hotelling para testar $H_{0}: \boldsymbol{R} \boldsymbol{\beta}=\boldsymbol{r}$ versus $H_{1}: \boldsymbol{R} \boldsymbol{\beta} \neq \boldsymbol{r}$ é dada por

$$
\xi_{w}^{*}=\left(\boldsymbol{R} \hat{\boldsymbol{\beta}}_{G}-\boldsymbol{r}\right)^{T}\left(\boldsymbol{R} \hat{\mathbf{V}}_{G} \boldsymbol{R}^{T}\right)^{-1}\left(\boldsymbol{R} \hat{\boldsymbol{\beta}}_{G}-\boldsymbol{r}\right),
$$

cuja distribuição nula assintótica é uma qui-quadrado central com $k$ graus de liberdade. Propomos, então, o seguinte procedimento para testar as hipóteses dadas em (5.2.5): rejeitar $H_{0}$ se $\xi_{w}^{*} \geq c_{2 \alpha}$ e $\sum_{j=1}^{k}\left(\boldsymbol{R}_{j}^{T} \hat{\boldsymbol{\beta}}_{G}-r_{j}\right)>0$, onde $\boldsymbol{R}_{j}^{T}$ denota a $j$-ésima linha de $\boldsymbol{R}, r_{j}$ é o $j$-ésimo elemento de $\boldsymbol{r}$ e $c_{2 \alpha}$ é tal que $P\left(\chi_{k}^{2} \geq c_{2 \alpha}\right)=2 \alpha$. Apresentaremos na Seção 5.4 alguns estudos de simulação em que comparamos o poder dos testes $\xi_{k p}, \xi_{w}^{+}$e $\xi_{w}^{*}$, através de EEGs para algumas estruturas de correlação e distribuições marginais.

\subsection{Aplicações}

\subsubsection{Previsão da safra de maçãs}

Com o objetivo de prever a safra de maçãs para cada produtor em uma determinada categoria, conforme exposto no Capítulo 1, Chen e Deely (1996) usando os dados de 207 produtores, apresentados no Apêndice D, construíram um modelo de regressão linear onde a variável resposta $y_{i}$ representa o número de caixas de maçãs produzidas pelo $i$-ésimo produtor e as variáveis regressoras são $x_{i j}$, o número de árvores na idade $j, j=2,3, \ldots, 11$, referente ao $i$-ésimo produtor, isto é,

$$
y_{i}=\sum_{j=2}^{11} \beta_{j} x_{i j}+\varepsilon_{i}, \quad \varepsilon_{i} \sim \mathrm{N}\left(0, \sigma^{2}\right) .
$$

Como os produtores admitem a priori que os coeficientes desse modelo devem ser restritos à forma $0 \leq \beta_{2} \leq \beta_{3} \cdots \leq \beta_{11}$, Chen e Deely (1996) compararam o método de 
mínimos quadrados ordinários e o método de estimação restrita apresentado em Liew (1976) com um método Bayesiano que leva em conta essa restrição de ordem. O nosso interesse, no entanto, é aplicar o procedimento de Follmann para verificar se os coeficientes desse modelo preservam a ordem aceita a priori pelos produtores, isto é, o interesse é testar $H_{0}: \beta_{2}=\beta_{3}=\cdots=\beta_{11}$ versus $H_{1}: \beta_{2} \leq \beta_{3} \leq \cdots \leq \beta_{11}$ com pelo menos uma desigualdade estrita. As estimativas não restritas dos parâmetros do modelo com os respectivos desvios padrão, obtidos pelo método de mínimos quadrados, são apresentadas na Tabela 5.3.1.

Tabela 5.3.1: Estimativas dos coeficientes do modelo para previsão da safra de maçãs com o respectivo desvio padrão.

\begin{tabular}{ccc}
\hline Coeficiente & Estimativa & Desvio padrão \\
\hline \hline$\beta_{2}$ & 0,09478 & 0,03229 \\
$\beta_{3}$ & $-0,02401$ & 0,01929 \\
$\beta_{4}$ & 0,19386 & 0,01211 \\
$\beta_{5}$ & 0,29421 & 0,04208 \\
$\beta_{6}$ & 0,53179 & 0,07831 \\
$\beta_{7}$ & 0,83362 & 0,03922 \\
$\beta_{8}$ & 0,74456 & 0,06559 \\
$\beta_{9}$ & 1,23486 & 0,44459 \\
$\beta_{10}$ & 0,52651 & 0,35547 \\
$\beta_{11}$ & 1,00189 & 0,89903 \\
\hline \hline
\end{tabular}

As hipóteses acima são equivalentes às hipóteses (5.2.5) onde $\boldsymbol{R}$ é uma matriz $(9 \times 10)$ cuja $i$-ésima linha tem o valor -1 na coluna $i$, o valor 1 na coluna $i+1$ e o valor zero nas outras colunas, $\boldsymbol{\beta}=\left(\beta_{2}, \beta_{3}, \ldots, \beta_{11}\right)^{T}$ e $\boldsymbol{r}$ é o vetor nulo $(9 \times 1)$. Para a estatística dada em (5.2.6), encontramos o valor $\xi_{w}^{*}=872,0581$ e $\sum_{j=1}^{9} \boldsymbol{R}_{j}^{T} \hat{\boldsymbol{\beta}}_{G}=0,9071>0$, portanto o nível descritivo para o teste de Follmann $\xi_{w}^{+}$, é menor do que 0,0001 . Isso indica 
uma forte evidência de que os coeficientes do modelo adotado para previsão da safra de maçãs, preservam a ordem admitida pelos produtores.

\subsubsection{Pressão arterial}

Vários experimentos clínicos realizados para medir o efeito da redução do sódio sobre a pressão do sangue em pacientes com pressão arterial normal são apresentados por Cutler et al. (1991). Em geral esses experimentos relatam separadamente o efeito sobre a pressão arterial sistólica e diastólica. Contudo, uma análise conjunta pode ser realizada para se ter uma única afirmação sobre a eficácia da redução do sódio sobre a pressão arterial. Se a redução do sódio afeta a pressão, espera-se uma redução tanto na pressão sistólica quanto na pressão diastólica, portanto, um teste unilateral multivariado pode ser apropriado nesse caso. A Tabela 5.3.2 apresenta a pressão média sistólica e diastólica com o respectivos desvios padrão para sete experimentos (Cutler et al., 1991).

Tabela 5.3.2: Redução na pressão arterial em sete experimentos clínicos.

\begin{tabular}{cc}
\hline \multicolumn{2}{c}{ Média da pressão arterial (mm Hg) (desvio padrão) } \\
\hline \hline Pressão sistólica & Pressão diastólica \\
\hline$-2.7(2.36)$ & $-3.0(2.03)$ \\
$-0.6(0.84)$ & $-1.4(1.07)$ \\
$-1.4(0.74)$ & $1.2(0.93)$ \\
$-0.5(0.82)$ & $1.4(0.90)$ \\
$-3.5(1.25)$ & $-1.9(0.90)$ \\
$-1.5(3.32)$ & $-1.1(2.03)$ \\
$0.1(1.00)$ & $0.2(0.80)$ \\
\hline
\end{tabular}


Ponderando a média de cada experimento pelo inverso da variância, as médias das pressões sistólica e diastólica $(\bar{P} s, \bar{P} d)$ para os sete experimentos e a respectiva matriz de covariância são dadas por

$$
\bar{P}=\left(\begin{array}{c}
\bar{P} s \\
\bar{P} d
\end{array}\right)=\left(\begin{array}{l}
-1.04 \\
-0.17
\end{array}\right) \text { e } \mathrm{V}_{\bar{P}}=\operatorname{Var}(\bar{P} s, \bar{P} s)=\left(\begin{array}{ll}
0.1510 & 0.0974 \\
0.0974 & 0.1524
\end{array}\right) .
$$

Para testar se esses efeitos são conjuntamente significantes, a estatística de Wald fornece o valor $\xi_{w}^{*}=\bar{P}^{T} \mathrm{~V}_{\bar{P}}^{-1} \bar{P}=9,97$, tem distribuição qui-quadrado central com 2 graus de liberdade e apresenta um $P$-ralor igual a 0,0068. Como a soma das médias das pressões sistólica e diastólica é negativa, o teste $\xi_{w}^{+}$apresenta um $P$-valor igual a 0,0034 . O teste de Follmann, portanto. produz uma evidência mais forte do efeito do sódio sobre a pressão arterial.

\subsection{Estudos de simulação}

Nestes estudos de simulação foram consideradas as mesmas condições das Seções 4.5, 4.6 e 4.7, ou seja, para as estruturas de correlação simétrica e $\operatorname{AR}(1)$ e $n=20,50$ e 100, foram geradas amostras independentes de $y_{i}=\left(y_{i 1}, y_{i 2}, y_{i 3}\right)^{T} \operatorname{com} y_{i j} \sim B\left(1, \mu_{i j}\right)$, $j=1,2,3$ e $i=1,2, \ldots, n$ amostras independentes de $y_{i}=\left(y_{i 1}, y_{i 2}, y_{i 3}, y_{i 4}\right)^{T}$ com $y_{i j} \sim \operatorname{Poisson}\left(\mu_{i j}\right), j=1,2,3,4$, e amostras independentes de $\boldsymbol{y}_{\boldsymbol{i}}=\left(y_{i 1}, y_{i 2}, y_{i 3}, y_{i 4}\right)^{T}$ $\operatorname{com} y_{i j} \sim \operatorname{Gama}\left(\mu_{i j}, \phi\right), \quad \phi=3, \quad j=1,2,3,4$, para $i=1,2, \ldots, n$

Para as estatísticas de Kodde e Palm e Wald, o poder foi estimado como sendo, respectivamente, a proporção de valores $\xi_{k p}$ e $\xi_{w}^{*}$, maiores que $c_{\alpha}$, o valor crítico assintótico correspondente ao nível de significância $\alpha$, para $\alpha=0,01 ; 0,05$ e 0,10 . O poder do teste de Follmann, $\xi_{w}^{+}$, foi estimado como sendo a proporção de valores $\xi_{w}^{*}$ satisfazendo $\xi_{w}^{*} \geq c_{2 \alpha}$ e $\sum_{j=1}^{k}\left(\boldsymbol{R}_{j}^{T} \hat{\boldsymbol{\beta}}_{G}-r_{j}\right)>0$, de acordo com o proposto no final da Seção 5.2. 
As Tabelas 5.4.1, 5.4 .2 e 5.4 .3 apresentam o poder estimado dos testes $\xi_{k p}, \xi_{w}^{*}$ e $\xi_{w}^{+}$, para distribuição binomial com estruturas de correlação simétrica e AR(1) e amostras de tamanho 20, 50 e 100, respectivamente. Os resultados para a distribuição de Poisson são apresentados nas Tabelas 5.4.4, 5.4.5 e 5.4.6, enquanto os resultados para a distribuição gama são apresentados nas Tabelas 5.4.7, 5.4 .8 e 5.4.9.

Quando $n=20$ e para todas as distribuições consideradas, o tamanho empírico dos testes $\xi_{k p}, \xi_{w}^{*}$ e $\xi_{w}^{+}$, são superiores ao tamanho nominal. No caso binomial com correlação $\operatorname{AR}(1)$ a diferença cresce com o aumento da correlação. Essa diferença diminui para a distribuição de Poisson com as duas estruturas de correlação e a distribuição gama não apresenta comportamento sistemático. Para $n=50$ e $n=100$, o tamanho empírico dos testes fica mais próximo do tamanho nominal para todas as distribuições consideradas e para as duas estruturas de correlação, ressaltando-se que, em geral, o tamanho empírico é mais próximo do tamanho nominal para o teste de Follmann.

O poder estimado dos testes decresce, em geral, com o aumento da correlação entre as observações da mesma unidade experimental, essa situação parece mais clara para a distribuição binomial com correlação simétrica e para a distribuição de Poisson com correlação $\mathrm{AR}(1)$. Novamente isso é explicado pelo fato das observações $y_{i j}(j=1,2,3,4)$ conterem pouca informação se forem altamente correlacionadas (vide Apêndice C).

É evidente o ganho de poder dos testes de Kodde e Palm e Follmann em relação ao teste de Wald, principalmente para amostras de tamanhos 50 e 100 . Isso é esperado pelo fato dos testes $\xi_{k p}$ e $\xi_{w}^{+}$levarem em conta as restrições sobre os parâmetros do modelo.

É importante notar que, embora haja um pequeno ganho de poder do teste de Kodde e Palm em relação ao teste de Follmann, este último é mais simples de ser aplicado uma vez que o mesmo não requer o cálculo dos pesos exigido pelo teste de Kodde e Palm. 
Isso torna o teste de Follmann um ótimo competidor, principalmente nos casos em que o número de restrições imposto pelas hipóteses é maior que 3. 


\section{Distribuição binomial com regressores}

Tabela 5.4.1: Binomial com regressores: poder das estatísticas $\xi_{k p}, \xi_{w}^{*}$ e $\xi_{w}^{+}$.

\begin{tabular}{|c|c|c|c|c|c|c|c|c|c|c|c|}
\hline \multicolumn{3}{|c|}{$n=20$} & \multicolumn{3}{|c|}{$\alpha=0,01$} & \multicolumn{3}{|c|}{$\alpha=0,05$} & \multicolumn{3}{|c|}{$\alpha=0,10$} \\
\hline$\rho$ & $\delta_{1}$ & $\delta_{2}$ & $\xi_{k p}$ & $\xi_{w}^{*}$ & $\xi_{w}^{+}$ & $\xi_{k p}$ & $\xi_{w}^{*}$ & $\xi_{w}^{+}$ & $\xi_{k p}$ & $\xi_{w}^{*}$ & $\xi_{w}^{+}$ \\
\hline \multicolumn{12}{|c|}{ Correlação simétrica } \\
\hline \multirow[t]{4}{*}{0,0} & 0,0 & 0,0 & ,057 & 0,080 & 0,040 & 0,138 & 0,168 & 0,106 & 0,204 & 5 &, 158 \\
\hline & 1,0 & 1,5 & & 0,248 & 0,213 & 0,435 & 0,378 &, 381 & & & \\
\hline & 1,5 & 2,0 & 0,271 & 0,305 & 0,252 & 0,521 & 0,431 & 8 & & &, 534 \\
\hline & 2.0 & 2,0 & 301 & 0,328 & 0,278 & 0,545 & 0,452 & 0,487 & 599 & 0,587 & 0,556 \\
\hline \multirow[t]{4}{*}{0,1} & 0,0 & 0,0 & & 0,071 & 0,039 & 0,128 & 0,162 & 0,093 &, 196 & 0,244 & 0,158 \\
\hline & 1,0 & 1,5 & 0 & 9 & 0,206 & 0,422 & 0.363 & 0,368 & 493 & 0,471 & 450 \\
\hline & 1,5 & 2,0 & .386 & 0,303 & 0,350 & 0,615 & 0.468 & 0,565 & & 580 & 691 \\
\hline & 2,0 & 2,0 & .417 & 0,320 & 0,370 & 0,630 & 0.490 & .581 & 744 & 0,596 & 0,698 \\
\hline \multirow[t]{4}{*}{0,3} & 0,0 & 0,0 & 58 & 81 & & 0,125 & 0,169 & & 88 & 0,240 & 0,166 \\
\hline & 1,0 & 1,5 & & & 188 & & & 0,345 & 453 & 0,465 & 0,412 \\
\hline & 1,5 & 2,0 & & 242 & ,272 & 0,532 & 0,382 & 0,477 & 641 & 0,504 & ,600 \\
\hline & 2,0 & 2,0 & 27 & 261 & 0,291 & 0,553 & 0,408 & 0,504 &, 677 & 0,531 & 627 \\
\hline \multirow[t]{4}{*}{0,7} & 0,0 & 0,0 & & 65 & &, 117 & 0,157 & 097 & 161 & 0,238 & 39 \\
\hline & 1,0 & 1,5 & & & & 33 & 6 & & & & 265 \\
\hline & 1,5 & 2,0 & & & $0,1 \varepsilon$ & & & & & 379 & 0,436 \\
\hline & 2,0 & 2,0 & & & 0,188 & 0,398 & 0,300 & 0,350 & 493 & 0,398 &, 449 \\
\hline \multicolumn{12}{|c|}{ Correlação AR(1) } \\
\hline \multirow[t]{4}{*}{0,0} & 0,0 & 0,0 & & 080 & 0,043 & & 0,174 & & & ,255 & 155 \\
\hline & 1,0 & ,5 & & & & & & 385 & & & 462 \\
\hline & 1,5 & 2 & & 305 & 0,251 & 0,523 & 0,434 & 456 & &, 567 & 530 \\
\hline & 2,0 & 2,0 & 05 & 329 & 0,285 & 0,548 & 0,456 & 489 & 602 & 0,592 & 550 \\
\hline \multirow[t]{4}{*}{0,1} & 0,0 & 0,0 & 53 & 0,077 & 0,040 & 0,134 & & & 12 & 0,252 & 0,160 \\
\hline & 1,0 & 1 & & 0,243 & & & 0,36 & 378 & & 0,482 & 456 \\
\hline & 1 , & 2 & & & & 0,517 & 0,438 & 0,454 & & 0,570 &, 523 \\
\hline & 2,0 & 2,0 & 98 & 0,325 & 0,280 & 0,535 & 0,443 & 0,479 & 0,582 & 0,586 & 0,534 \\
\hline \multirow[t]{4}{*}{0,3} & 0,0 & 0,0 & 68 & 097 & & 0,146 & 0,186 & 0,113 & & 262 & 170 \\
\hline & 1,0 & 1,5 & & & 0,2 & & & & & 0,513 & 442 \\
\hline & & 2,0 & & & & 0,485 & 0,405 & 0,436 & & 0,546 & 0,494 \\
\hline & 2,0 & 2,0 & & 0,308 & 0,264 & 0,503 & 0,420 & 0,450 & 0,543 & 0,548 & 0,496 \\
\hline \multirow[t]{4}{*}{0,7} & 0,0 & 0,0 & & & & & 0,200 & & 0,224 & 0,304 & 0,185 \\
\hline & & & & & & 0,406 & 0,376 & & & 500 & 399 \\
\hline & 1,5 & 2,0 & & 0,267 & 0,229 & 0,401 & & & & 0,458 &, 399 \\
\hline & 2,0 & 2,0 & 0,250 & 0,269 & 0,236 & 0,409 & 0,349 & 0,374 & 0,431 & 0,449 & 0,396 \\
\hline
\end{tabular}


Tabela 5.4.2: Binomial com regressores: poder das estatísticas $\xi_{k p}, \xi_{w}^{*}$ e $\xi_{w}^{+}$.

\begin{tabular}{|c|c|c|c|c|c|c|c|c|c|c|c|}
\hline \multicolumn{3}{|c|}{$n=50$} & \multicolumn{3}{|c|}{$\alpha=0,01$} & \multicolumn{3}{|c|}{$\alpha=0,05$} & \multicolumn{3}{|c|}{$\alpha=0,10$} \\
\hline$\rho$ & $\delta_{1}$ & $\delta_{2}$ & $\xi_{k p}$ & $\xi_{w}^{*}$ & $\xi_{w}^{+}$ & $\xi_{k p}$ & $\xi_{w}^{*}$ & $\xi_{w}^{+}$ & $\xi_{k p}$ & $\xi_{w}^{*}$ & $\xi_{w}^{+}$ \\
\hline \multicolumn{12}{|c|}{ Correlação simétrica } \\
\hline \multirow[t]{4}{*}{0,0} & 0,0 & 0,0 & 0,014 & 0,016 & 0,009 & $\overline{0,061}$ & 0,075 & 0,049 & 0,123 & 0,126 & 0,098 \\
\hline & 1,0 & 1,5 & 0,186 & 0,127 & 0,162 & 0,412 & 0,271 & 0,381 & 0,549 & 0,401 & 0,497 \\
\hline & 1,5 & 2,0 & 0,283 & 0,210 & 0,245 & 0,528 & 0,377 & 0,485 & 0,669 & 0,499 & 0,626 \\
\hline & 2,0 & 2,0 & 0,362 & 0,261 & 0,322 & 0,595 & 0,441 & 0,565 & 0,729 & 0,581 & 0,693 \\
\hline \multirow[t]{4}{*}{0,1} & 0,0 & 0,0 & 0,009 & 0,021 & 0,007 & 0,060 & 0,067 & 0,043 & 0,127 & 0,128 & 0,100 \\
\hline & 1,0 & 1,5 & 0,181 & 0,121 & 0,153 & 0,403 & 0,268 & 0,366 & 0,536 & 0,391 & 0,493 \\
\hline & 1,5 & 2,0 & 0,285 & 0,204 & 0,248 & 0,528 & 0,382 & 0,489 & 0,656 & 0,507 & 0,624 \\
\hline & 2,0 & 2,0 & 0,348 & 0,253 & 0,312 & 0,580 & 0,423 & 0,544 & 0,716 & 0,562 & 0,686 \\
\hline \multirow{4}{*}{0,3} & 0,0 & 0,0 & 0,015 & 0,018 & 0,008 & 0,068 & 0,080 & 0,057 & 0,138 & 0,145 & 0,113 \\
\hline & 1,0 & 1,5 & 0,169 & 0,118 & 0,147 & 0,384 & 0,262 & ,342 & 0,517 & 0,377 & 0,476 \\
\hline & 1,5 & 2,0 & 0,281 & 0,207 & 0,244 & 0,515 & 0,381 & 0,474 & 0,652 & 0,502 & 0,617 \\
\hline & 2,0 & 2,0 & 0,340 & 0,254 & 0,297 & 0,556 & 0,429 & 0,507 & 0,701 & 0,536 & 0,663 \\
\hline \multirow[t]{4}{*}{0,7} & 0,0 & 0,0 & 0,017 & 0,027 & 0,010 & 0,085 & 0,099 & 0,072 & 0,151 & 0,180 & 0,130 \\
\hline & 1,0 & 1,5 & 0,178 & 0,142 & 0,155 & 0,359 & 0,279 & 0,313 & 0,468 & 0,372 & 0,422 \\
\hline & 1,5 & 2,0 & 0,246 & 0,214 & 0,216 & 0,451 & 0,361 & 0,400 & 0,555 & 0,465 & 0,510 \\
\hline & 2,0 & 2,0 & 0,286 & 0,236 & 0,257 & 0,449 & 0,376 & 0,416 & 0,559 & 0,468 & 0,520 \\
\hline \multicolumn{12}{|c|}{ Correlação AR(1) } \\
\hline \multirow[t]{4}{*}{0,0} & 0,0 & 0,0 & 0,012 & 0,018 & 0,009 & 0,063 & 0,073 & 0,051 & 0,124 & 0,127 & 0,098 \\
\hline & 1,0 & 1,5 & 0,190 & 0,129 & 0,166 & 0,415 & 0,275 & 0,385 & 0,543 & 0,403 & 0,496 \\
\hline & 1,5 & 2,0 & 0,280 & 0,208 & 0,246 & 0,532 & 0,381 & 0,478 & 0,670 & 0,493 & 0,630 \\
\hline & 2,0 & 2,0 & 0,358 & 0,265 & 0,323 & 0,601 & 0,441 & 0,562 & 0,729 & 0,579 & 0,695 \\
\hline \multirow[t]{4}{*}{0,1} & 0,0 & 0,0 & 0,011 & 0,023 & 0,008 & 0,057 & 0,069 & 0,040 & 0,125 & 0,118 & 0,096 \\
\hline & 1,0 & 1,5 & 0,186 & 0,124 & 0,157 & 0,405 & 0,276 & 0,363 & 0,539 & 0,390 & 0,492 \\
\hline & 1,5 & 2,0 & 0,295 & 0,206 & 0,251 & 0,528 & 0,394 & 0,486 & 0,661 & 0,505 & 0,662 \\
\hline & 2,0 & 2,0 & 0,353 & 0,267 & 0,322 & 0,584 & 0,431 & 0,545 & 0,719 & 0,568 & 0,685 \\
\hline \multirow[t]{4}{*}{0,3} & 0,0 & 0,0 & 0,012 & 0,018 & 0,008 & 0,072 & 0,081 & 0,055 & 0,142 & 0,149 & 0,120 \\
\hline & 1,0 & 1,5 & 0,176 & 0,122 & 0,146 & 0,381 & 0,273 & 0,337 & 0,518 & 0,372 & 0,475 \\
\hline & 1,5 & 2,0 & 0,289 & 0,214 & 0,244 & 0,524 & 0,385 & 0,476 & 0,651 & 0,503 & 0,619 \\
\hline & 2,0 & 2,0 & 0,351 & 0,262 & 0,310 & 0,581 & 0,441 & 0,525 & 0,701 & 0,552 & 0,666 \\
\hline \multirow[t]{4}{*}{0,7} & 0,0 & 0,0 & 0,017 & 0,028 & 0,015 & 0,085 & 0,087 & 0,068 & 0,149 & 0,164 & 0,123 \\
\hline & 1,0 & 1,5 & 0,216 & 0,147 & 0,172 & 0,400 & 0,315 & 0,356 & 0,528 & 0,418 & 0,482 \\
\hline & 1,5 & 2,0 & 0,302 & 0,244 & 0,273 & 0,504 & 0,420 & 0,468 & 0,637 & 0,525 & 0,591 \\
\hline & 2,0 & 2,0 & 0,363 & 0,290 & 0,324 & 0,561 & 0,476 & 0,515 & 0,680 & 0,563 & 0,641 \\
\hline
\end{tabular}


Tabela 5.4.3: Binomial com regressores: poder das estatísticas $\xi_{k p}, \xi_{w}^{*}$ e $\xi_{w}^{+}$.

\begin{tabular}{|c|c|c|c|c|c|c|c|c|c|c|c|}
\hline \multicolumn{3}{|c|}{$n=100$} & \multicolumn{3}{|c|}{$\alpha=0,01$} & \multicolumn{3}{|c|}{$\alpha=0,05$} & \multicolumn{3}{|c|}{$\alpha=0,10$} \\
\hline$\rho$ & $\delta_{1}$ & $\delta_{2}$ & $\xi_{k p}$ & $\xi_{w}^{*}$ & $\xi_{w}^{+}$ & $\xi_{k p}$ & $\xi_{w}^{*}$ & $\xi_{w}^{+}$ & $\xi_{k p}$ & $\xi_{w}^{*}$ & $\xi_{w}^{+}$ \\
\hline \multicolumn{12}{|c|}{ Correlação simétrica } \\
\hline \multirow[t]{4}{*}{0,0} & 0,0 & 0,0 & 0,016 & 0,020 & 0,012 & 0,060 & 0,067 & 0,050 & 0,118 & 0,117 & 0,096 \\
\hline & 1,0 & 1,5 & 0,271 & 0,148 & 0,222 & 0,549 & 0,360 & & 679 &, 488 & 634 \\
\hline & 1,5 & 2,0 & 0,362 & 0,235 & 0,322 & 0,659 & 0,473 & 0,620 & 774 & 626 & 0,747 \\
\hline & 2,0 & 2,0 & 0,420 & 0,309 & 0,386 & 0,696 & 0,528 & 0,665 & 0,800 & 0,669 & 0,783 \\
\hline \multirow[t]{4}{*}{0,1} & 0,0 & 0,0 & 0,0 & 0,023 & 0,010 & 0,058 & 0,069 & 0,051 & 0,118 &, 124 & 0,105 \\
\hline & 1,0 & 1,5 & 251 & 0,135 & 0,202 & 0,518 & 0,333 & & 0,665 & 469 & 0,618 \\
\hline & 1,5 & 2,0 & 0,3 & 0,220 & 0,310 & 0,615 & 0,454 & 580 &, 762 & 586 &, 738 \\
\hline & 2,0 & 2,0 & 397 & 0,284 & 0,354 & 0,662 & 0,514 & 0,629 & 783 & 635 & 0,757 \\
\hline \multirow[t]{4}{*}{0,3} & 0,0 & 0,0 & 007 & 0,018 & 0,006 & 0,069 & 0,071 & & 20 & 137 & 0,107 \\
\hline & 1,0 & 1,5 & 28 & 135 & 189 & 0,493 & 0,317 & & & & 0,585 \\
\hline & 1,5 & 2,0 & 27 & 0,215 & 0,295 & 0,586 & 0,436 & & 0,718 & 566 & 0,679 \\
\hline & 2,0 & 2,0 & 0,37 & 0,258 & 0,332 & 0,634 & 0,468 & 0,558 & 767 & 601 & 0,729 \\
\hline \multirow[t]{4}{*}{0,7} & 0,0 & 0,0 & 5 & 0,022 & & 0,068 & 0,078 & & & & 0,106 \\
\hline & 1,0 & 1,5 & 189 & 115 & & & 0,283 & & & & 502 \\
\hline & 1,5 & 2,0 & & 204 & & 0,528 & 0,397 & & & & 0,611 \\
\hline & 2,0 & 2,0 & & 250 & 0,280 & 0,568 & 0,427 & & & & 0,640 \\
\hline \multicolumn{12}{|c|}{ Correlação AR(1) } \\
\hline \multirow[t]{4}{*}{0,0} & 0,0 & 0,0 & & & 0,012 & 0,060 & 0,068 & & & & 095 \\
\hline & 1,0 & 1,5 & & 149 & & 0,520 & 0,357 & & 662 & & 0,632 \\
\hline & 1,5 & 2,0 & & 236 & 0,322 & 0,65 & 0,469 & 0,624 & & & 0,747 \\
\hline & 2,0 & 2,0 & 22 & 310 & 0,389 & 0,697 & 0,527 & 0,663 & & & 0,785 \\
\hline \multirow[t]{4}{*}{0,1} & 0,0 & 0,0 & & 025 & 0,015 & 0,067 & 0,066 & 0,052 & & 22 & 0,102 \\
\hline & 1,0 & 1,5 & & 140 & 0,203 & 0,499 & 0,335 & & 40 & & 0,612 \\
\hline & 1,5 & 2,0 & & & & 0,625 & 0,457 & 0,5 &, 764 & & 0,741 \\
\hline & 2,0 & 2,0 & & 284 & 0,362 & 0,667 & 0,502 & 0,623 & 785 & 0,628 & 0,761 \\
\hline \multirow[t]{4}{*}{0,3} & 0,0 & 0,0 & 09 & 017 & 0,008 & 0,067 & 0,071 & 0,056 & & & 0,112 \\
\hline & 1,0 & 1,5 & & & & & & & & & 0,574 \\
\hline & 1,5 & 2,0 & & & & 0,584 & 0,430 & & 0,718 & & 0,679 \\
\hline & 2,0 & 2,0 & & 0,252 & 0,328 & 0,633 & 0,461 & 0,595 & 0,757 & 0,605 & 0,733 \\
\hline \multirow[t]{4}{*}{0,7} & 0,0 & 0,0 & & 0,028 & & 0,062 & & & & & 0,098 \\
\hline & 1,0 & & & & & & 276 & & & 382 & 0,501 \\
\hline & 1, & & & & & & 0,393 & & & & 0,624 \\
\hline & 2,0 & 2,0 & 0,331 & 0,242 & 0,296 & 0,582 & 0,429 & 0,524 & 0,712 & 0,551 & 0,677 \\
\hline
\end{tabular}




\section{Distribuição de Poisson com regressores}

Tabela 5.4.4: Poisson com regressores: poder das estatísticas $\xi_{k p}, \xi_{w}^{*}$ e $\xi_{w}^{+}$.

\begin{tabular}{|c|c|c|c|c|c|c|c|c|c|c|c|}
\hline \multicolumn{3}{|c|}{$n=20$} & \multicolumn{3}{|c|}{$\alpha=0,01$} & \multicolumn{3}{|c|}{$\alpha=0,05$} & \multicolumn{3}{|c|}{$\alpha=0,10$} \\
\hline$\rho$ & $\delta_{1}$ & $\delta_{2}$ & $\xi_{k p}$ & $\xi_{w}^{*}$ & $\xi_{w}^{+}$ & $\xi_{k p}$ & $\xi_{w}^{*}$ & $\xi_{w}^{+}$ & $\xi_{k p}$ & $\xi_{w}^{*}$ & $\xi_{w}^{+}$ \\
\hline \multicolumn{12}{|c|}{ Correlação simétrica } \\
\hline \multirow[t]{4}{*}{0,0} & 0,0 & 0,0 & 0,056 & 0,082 & 0,043 & 0,119 & 0,166 & 0,091 & 0,184 & 0,234 & 0,139 \\
\hline & 0,0 & 0,2 & 208 & 0,185 & 0,187 & 0,349 & 0,291 &, 303 &, 475 & 0,362 &, 424 \\
\hline & 0,2 & 0,0 & 424 & 0,339 & 0,368 & 0,606 & 0,527 & 0,546 &, 703 & 0,629 &, 627 \\
\hline & 0,2 & 0,2 & 0,663 & 0,553 & 0,618 & 0,838 & 0,735 & 0,801 & 0,903 & 0,810 & 0,875 \\
\hline \multirow[t]{4}{*}{0,1} & 0,0 & 0,0 & & 0,097 & 0,049 & 0,138 & 0,165 & 110 & 209 &, 251 & 0,173 \\
\hline & 0,0 & 0,2 & 221 & 0,202 & 0.197 & 0,362 & 0,315 & 320 & ,481 & 390 &, 423 \\
\hline & 0,2 & 0,0 & 387 & 0,313 & 0.337 & 0,578 & 0,477 & 519 & 0,693 & 582 &, 628 \\
\hline & 0,2 & 0,2 & 0,644 & 0,546 & 0,607 & 0,840 & 0,725 & 0,808 & 0,916 & 0,818 & 0,881 \\
\hline \multirow[t]{4}{*}{0,3} & 0,0 & 0,0 & 43 & 0,086 & 0,035 & 0,103 & 0,184 & 0,079 &, 166 & 256 &, 122 \\
\hline & 0,0 & 0,2 & & & & & 321 & 58 & & 415 & 447 \\
\hline & 0,2 & 0,0 & & 0,318 & 0,319 & 0,567 & 0,484 & 500 & 693 & 579 & ,602 \\
\hline & 0,2 & 0,2 & 634 & 0,532 & 0,603 & 0,822 & 0,710 & 0,783 & 0,892 & 0,791 & 0,864 \\
\hline \multirow[t]{4}{*}{0,7} & 0,0 & 0,0 & 46 & 0,094 & 0,032 & 0,103 & 0,185 & 0,073 & 0,168 &, 250 & 0,117 \\
\hline & 0,0 & 0,2 & 16 & 183 & 193 & 0,370 & 0,310 & 319 & 0,479 & 396 &, 431 \\
\hline & 0,2 & 0,0 & & 307 & 0,319 & 0,571 & 0,475 & 00 & 675 & 594 &, 596 \\
\hline & 0,2 & 0,2 & & 0,532 & 0,586 & 0,801 & 0,689 & 0,764 & 0,874 &, 770 & 0,845 \\
\hline \multicolumn{12}{|c|}{ Correlação AR(1) } \\
\hline \multirow[t]{4}{*}{0,0} & 0,0 & 0,0 & & , 089 & 0,040 & 0,126 & 0,167 & 0,095 &, 185 & ,238 & 0,141 \\
\hline & 0,0 & 0,2 & & 0,184 & & & 0,293 & 306 & 171 & 364 & 0,418 \\
\hline & 0,2 & 0,0 & 421 & 0,346 & 0,372 & 0,611 & 0,533 & 0,539 & 0,703 & 0,623 & 0,631 \\
\hline & 0,2 & 0,2 & 672 & 0,553 & 0,623 & 0,840 & 0,734 & 0,810 & 0,903 & 0,816 & 0,879 \\
\hline \multirow[t]{4}{*}{0,1} & 0,0 & 0,0 & 068 & 0,106 & 0,051 & 0,159 & 0,203 & 0,125 & 0,235 & 0,274 &, 181 \\
\hline & 0,0 & 0,2 & & 0,183 & & & 0,304 & 15 & 157 & ,387 & 0,413 \\
\hline & 0,2 & 0,0 & & 0,305 & & & 0,467 & 0,495 & 0,688 & 0,574 & 0,602 \\
\hline & 0,2 & 0,2 & 0,576 & 0,468 & 0,539 & 0,781 & 0,650 & 0,735 & 0,860 & 0,744 & 0,828 \\
\hline \multirow[t]{4}{*}{0,3} & 0,0 & 0,0 & 0,063 & 0,093 & 0,049 & 0,139 & 0,177 & 0,114 & 0,197 & 0,255 &, 165 \\
\hline & 0,0 & 0,2 & & 165 & 0,159 & 0,338 & 0,298 & 0,290 & 0,454 & 0,383 &, 389 \\
\hline & 0,2 & 0,0 & & 0,262 & 0,262 & & 0,418 & 0,409 & 0,584 & 0,508 & 0,501 \\
\hline & 0,2 & 0,2 & 0,495 & 0,388 & 0,454 & 0,702 & 0,576 & 0,653 & 0,798 & 0,665 & 0,759 \\
\hline \multirow[t]{4}{*}{0,7} & 0,0 & 0,0 & 54 & 0,078 & 0,045 & 0,118 & 0,168 & 0,099 & 0,191 & 0,245 & 0,153 \\
\hline & 0,0 & 0,2 & & & & 0,268 & 0,225 & & 0,352 &, 308 & 0,317 \\
\hline & 0,2 & 0,0 & & 0,168 & 0,159 & 0,380 & 0,306 & 0,3 & 0,492 & 0,397 & 0,415 \\
\hline & 0,2 & 0,2 & 0,348 & 0,268 & 0,308 & 0,551 & 0,438 & 0,493 & 0,665 & 0,528 & 0,610 \\
\hline
\end{tabular}


Tabela 5.4.5: Poisson com regressores: poder das estatísticas $\xi_{k p}, \xi_{w}^{*}$ e $\xi_{w}^{+}$.

\begin{tabular}{|c|c|c|c|c|c|c|c|c|c|c|c|}
\hline \multicolumn{3}{|c|}{$n=50$} & \multicolumn{3}{|c|}{$\alpha=0,01$} & \multicolumn{3}{|c|}{$\alpha=0,05$} & \multicolumn{3}{|c|}{$\alpha=0,10$} \\
\hline$\rho$ & $\delta_{1}$ & $\delta_{2}$ & $\xi_{k p}$ & $\xi_{w}^{*}$ & $\xi_{w}^{+}$ & $\xi_{k p}$ & $\xi_{w}^{*}$ & $\xi_{w}^{+}$ & $\xi_{k p}$ & $\xi_{w}^{*}$ & $\xi_{w}^{+}$ \\
\hline \multicolumn{12}{|c|}{ Correlação simétrica } \\
\hline \multirow[t]{4}{*}{0,0} & 0,0 & 0,0 & 0,008 & 0,026 & 0.005 & 0,056 & 0,079 & 0,040 & 0,000 & 0,143 & 0,073 \\
\hline & 0,0 & 0,2 & 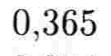 & 0,308 & 0.339 & 0,611 & 0,510 & 0,567 & , & 0,628 & 688 \\
\hline & 0,2 & 0,0 & 0,374 & 0,302 & 0,344 & 0,616 & 0,515 & 576 & & & 686 \\
\hline & 0,2 & 0,2 & 0,842 & 0,745 & 0.814 & 0,947 & 0,893 & 935 & & 0,935 & 0,972 \\
\hline \multirow[t]{4}{*}{0,1} & 0,0 & 0,0 & & 0,030 & 0,015 & 0,062 & 0,098 & 0,049 & 0,125 & 0,164 &, 104 \\
\hline & 0,0 & 0,2 & 396 & 330 & 0.366 & 0,606 & 0,524 & 0,574 & 0,731 & 0,618 & 0,696 \\
\hline & 0,2 & 0,0 & 0,3 & 0,3 & 0.336 & 0,628 & 0,529 & 0,581 & 0,746 & 0,651 & 712 \\
\hline & 0,2 & 0,2 & 0,810 & 0,719 & 0.790 & 0,943 & 0,875 & 0,923 & & 923 &, 964 \\
\hline \multirow[t]{4}{*}{0,3} & 0,0 & 0 & 023 & 0,031 & 0.017 & 0,073 & 0,088 & 0,058 & & 163 &, 105 \\
\hline & 0,0 & & & & 360 & 13 & 0 & & & 0,619 & 0,697 \\
\hline & 0,2 & 0,0 & & 0,314 & 0,360 & 0,6 & 0,527 & 0,576 & 0,732 & 0,633 &, 698 \\
\hline & 0,2 & 0,2 & 0,818 & 0,709 & 0,788 & 0,953 & 0,886 & 0,937 & 0,977 &, 937 & 0,973 \\
\hline \multirow[t]{4}{*}{0,7} & 0,0 & 0,0 & 0,023 & 039 & & 0,065 & 0,100 & & & 148 & 0,090 \\
\hline & 0,0 & 0,2 & 383 & 328 & & & 528 & & & & 676 \\
\hline & 0,2 & 0,0 & 412 & 332 & 0,377 & 0,633 & 0,544 & & & & 0,710 \\
\hline & 0,2 & 0,2 & 819 & 713 & 0,786 & 0,941 & 0,881 & & & & 972 \\
\hline \multicolumn{12}{|c|}{ Correlação AR(1) } \\
\hline \multirow[t]{4}{*}{0,0} & 0,0 & & & 026 & 0,005 & 0,056 & 0,076 & & & & \\
\hline & 0,0 & & 364 & 304 & & 0,608 & 0,514 & & 4 &, 630 & \\
\hline & 0,2 & & 377 & 308 & & 0,616 & 0,520 & 73 & 27 & 0,625 & 688 \\
\hline & 0, & 0 & 842 & 746 & 0,814 & 0,945 & 0,891 & 0,937 & & 938 & 0,971 \\
\hline \multirow[t]{4}{*}{0,1} & 0,0 & 0,0 & 026 & 035 & 0,023 & 0,060 & 0,117 & & & 174 & 0,086 \\
\hline & 0,0 & & & 266 & 0,303 & & 0,4 & 09 & & & 0,639 \\
\hline & 0,2 & 0,0 & & & & 0,569 & 0,491 & 0,526 & 89 & 0,603 & 0,640 \\
\hline & 0,2 & & 764 & 658 & 0,721 & 0,899 & 0,826 & 0,883 & 0,943 & 0,885 &, 934 \\
\hline \multirow[t]{4}{*}{0,3} & 0,0 & & 031 & 039 & 0,025 & 0,074 & 0,103 & 0,066 & & 0,170 & 0,107 \\
\hline & 0,0 & & & & 0,257 & & & & & 496 & 0,581 \\
\hline & 0,2 & & & & 0,241 & 0,481 & 0,386 & & & ,499 & 0,581 \\
\hline & 0,2 & & 648 & 0,532 & 0,601 & 0,828 & 0,732 & 0,803 & 0,916 & 0,804 & 0,897 \\
\hline \multirow[t]{4}{*}{0,7} & 0,0 & & 032 & 0,038 & 0,028 & 0,081 & & & & & 0,108 \\
\hline & & & & & & & 0,293 & & & 0,384 & 0,440 \\
\hline & & & & & & & 0,277 & & & 0,388 & 0,445 \\
\hline & 0,2 & 0,2 & 0,432 & 0,310 & 0,388 & 0,654 & 0,516 & 0,608 & 0,763 & 0,612 & 0,735 \\
\hline
\end{tabular}


Tabela 5.4.6: Poisson com regressores: poder das estatísticas $\xi_{k p}, \xi_{w}^{*}$ e $\xi_{w}^{+}$.

\begin{tabular}{|c|c|c|c|c|c|c|c|c|c|c|c|}
\hline \multicolumn{3}{|c|}{$n=100$} & \multicolumn{3}{|c|}{$\alpha=0,01$} & \multicolumn{3}{|c|}{$\alpha=0,05$} & \multicolumn{3}{|c|}{$\alpha=0,10$} \\
\hline$\rho$ & $\delta_{1}$ & $\delta_{2}$ & $\xi_{k p}$ & $\xi_{w}^{*}$ & $\xi_{w}^{+}$ & $\xi_{k p}$ & $\xi_{w}^{*}$ & $\xi_{w}^{+}$ & $\xi_{k p}$ & $\xi_{w}^{*}$ & $\xi_{w}^{+}$ \\
\hline \multicolumn{12}{|c|}{ Correlação simétrica } \\
\hline \multirow[t]{4}{*}{0,0} & 0,0 & 0,0 & 0,009 & 0,017 & 0,005 & 0,049 & 0,072 & 0,038 &, 089 & 0,123 & 0,078 \\
\hline & 0,0 & 0,2 & 612 & 0,536 & 0,574 & 0,816 & 0,756 & 0,796 & ,884 &, 849 &, 864 \\
\hline & 0,2 & 0,0 & 678 & 0,586 & 0,778 & 0,839 & 0,778 & 0,821 &, 912 & 0,852 & 0,893 \\
\hline & 0,2 & 0,2 & 930 & 0,872 & 0,923 & 0,980 & 0,960 & 0.978 & 0,995 & 0,978 & 0,992 \\
\hline \multirow[t]{4}{*}{0,1} & 0.0 & & 011 & 0,025 & 0,010 & 0,045 & 0,071 & 0,038 & 095 & 0,119 &, 079 \\
\hline & 0,0 & 2 & & 514 & 551 & 0,796 & 0,727 & 771 & 881 & 816 & 863 \\
\hline & 0,2 & 0,0 & 661 & 0,604 & 0,632 & 0,850 & 0,786 & 0,830 &, 900 & 0,858 & 0,883 \\
\hline & 0.2 & 0,2 & 0.939 & 0,888 & 0,929 & 0,985 & 0,959 & 0.981 & 0.997 & 0,981 & 0,996 \\
\hline \multirow[t]{4}{*}{0,3} & 0.0 & 0,0 & 0.016 & 0,027 & 0,013 & 0,059 & 0,086 & 0,051 & 107 & 0,148 & $\overline{0,090}$ \\
\hline & 0.0 & & & 489 & & 0,777 & 0,696 & 0,751 & & 0,788 & 843 \\
\hline & 0,2 & 0,0 & & 588 & 0,6 & 0,859 & 0,798 & 842 & 918 & 0,878 & 0,900 \\
\hline & 0,2 & 0,2 & 0,936 & 0,875 & 0,920 & 0,986 & 0,963 & 0,983 &, 994 & 0,983 & 0,994 \\
\hline \multirow[t]{4}{*}{0,7} & 0,0 & 0,0 & 0,019 & 0,032 & 0,014 & 0,073 & 0,099 & 0,059 & 0,107 & 0,160 & 0,096 \\
\hline & 0,0 & & & 0,467 & 0,514 & 0,771 & 0,692 & 0,742 & ),860 & 0,794 &, 839 \\
\hline & 0,2 & & & 619 & 0,667 & 0,8 & 0,796 & 0,831 & 927 & 0,876 & 0,903 \\
\hline & 0,2 & & & 857 & 0,914 & 0,980 & 0,960 & 0,977 & 0,996 & 0,977 & 0,994 \\
\hline \multicolumn{12}{|c|}{ Correlação AR(1) } \\
\hline \multirow[t]{4}{*}{0,0} & 0,0 & & & & 005 & 0,047 & 0,072 & & &, 127 & 0,079 \\
\hline & 0,0 & 0,2 & 3 & 0,538 & 0,5 & 0,8 & 0,757 & 0,796 & & ,848 & ), 866 \\
\hline & 0,2 & 0,0 & 0,679 & 0,585 & 0,646 & 0,8 & 0,775 & 0,819 & 0,912 & 0,852 & 0,894 \\
\hline & 0,2 & 0,2 & 0,930 & 0,875 & 0,922 & 0,981 & 0,960 & 0,975 & 0,995 & 0,975 & 0,992 \\
\hline \multirow[t]{4}{*}{0,1} & 0,0 & 0,0 & 081 & 0,021 & 0,01 & 0,052 & 0,078 & 0,044 & 0,089 & 0,132 & 0,076 \\
\hline & 0,0 & 0,2 & & 461 & & 0,7 & 686 & 51 & & 0,794 & 0,839 \\
\hline & 0,2 & 0,0 & & 0,496 & & 0,796 & 0,729 & 0,764 & & 0,818 & 0,846 \\
\hline & 0,2 & 0,2 & 0,898 & 0,835 & 0,874 & 0,965 & 0,938 & 0,960 & 0,985 & 0,960 & 0,984 \\
\hline \multirow[t]{4}{*}{0,3} & 0,0 & 0,0 & 0,015 & 0,024 & 0,013 & 0,068 & 0,084 & 0,054 & 0,113 & 0,142 & 0,095 \\
\hline & 0,0 & 0,2 & & 304 & 0,337 & 0,6 & 0,520 & 0,598 & & 0,640 & 0,715 \\
\hline & 0,2 & 0,0 & & & & & 0,605 & & & 0,713 & 0,791 \\
\hline & 0,2 & 0,2 & 0,805 & 0,696 & 0,783 & 0,933 & 0,875 & 0,923 & 0,966 & 0,923 & 0,959 \\
\hline \multirow[t]{4}{*}{0,7} & 0,0 & 0,0 & 0,011 & 0,018 & 0,010 & 0,068 & 0,064 & 0,056 & 0,112 & 0,113 & 0,101 \\
\hline & 0,0 & & & 0,187 & 0,218 & 0,470 & 0,347 & 0,426 & & 0,473 & 0,574 \\
\hline & 0,2 & & & & & 0,534 & 0,445 & 0,507 & 0,681 & 0,571 & 0,645 \\
\hline & 0,2 & 0,2 & 0,569 & 0,462 & 0,539 & 0,785 & 0,671 & 0,767 & 0,874 & 0,770 & 0,863 \\
\hline
\end{tabular}




\section{Distribuição gama com regressores}

Tabela 5.4.7: Gama com regressores: poder das estatísticas $\xi_{k p}, \xi_{w}^{*}$ e $\xi_{w}^{+}$.

\begin{tabular}{|c|c|c|c|c|c|c|c|c|c|c|c|}
\hline \multicolumn{3}{|c|}{$n=20$} & \multicolumn{3}{|c|}{$\alpha=0,01$} & \multicolumn{3}{|c|}{$\alpha=0,05$} & \multicolumn{3}{|c|}{$\alpha=0,10$} \\
\hline$\rho$ & $\delta_{1}$ & $\delta_{2}$ & $\xi_{k p}$ & $\xi_{w}^{*}$ & $\xi_{w}^{+}$ & $\xi_{k p}$ & $\xi_{w}^{*}$ & $\xi_{w}^{+}$ & $\xi_{k p}$ & $\xi_{w}^{*}$ & $\xi_{w}^{+}$ \\
\hline \multicolumn{12}{|c|}{ Correlação simétrica } \\
\hline \multirow[t]{4}{*}{0,0} & 0,0 & 0,0 & 0,068 & 0,101 & 0,044 & 0,142 & 0,193 & 0,110 & & & 0,156 \\
\hline & 0,0 & 0,5 & & 0 , & 0,133 & 0,292 & 0,251 & & & 0,338 & 0,337 \\
\hline & 0,5 & 0,0 & 0,225 & 0,203 & 0,185 & 0,396 & 0,340 & 0,330 & 0,512 & 432 & 428 \\
\hline & 0,5 & 0,5 & 310 & 0,245 & 0,270 & 0,512 & 0,396 & 0,452 & 0,631 & &, 579 \\
\hline \multirow[t]{4}{*}{0,1} & 0,0 & 0,0 & 0,052 & 0,079 & 0,0 & & 0,180 & & 174 & 249 & 132 \\
\hline & 0,0 & 0,5 & 127 & 128 & & 0,261 & 0,227 & 0,227 & & 321 & 316 \\
\hline & 0,5 & 0,0 & 213 & 0,137 & 0,172 & 0,388 & 0,330 & 12 & 0,50 & 0,413 & 0,420 \\
\hline & 0,5 & 0,5 & 0,2 & 0,226 & 0,259 & 0,524 & 0,379 & 0,476 & 0,633 & 0,511 &, 588 \\
\hline \multirow[t]{4}{*}{0,3} & 0,0 & 0,0 &, 051 & 0,087 & 0,038 & 0,114 & 0,181 & & 179 & & 126 \\
\hline & 0,0 & 0,5 & & 0,143 & & 8 & 0,260 & & & & 370 \\
\hline & 0,5 & 0,0 & 267 & 0,229 & & 0,464 & 0,384 & & & & 494 \\
\hline & 0,5 & 0,5 & 396 & 292 & 0,346 & 0,611 & 0,464 & 0,560 & 727 & 589 &, 674 \\
\hline \multirow[t]{5}{*}{0,7} & 0,0 & 0,0 & 664 & 0,102 & 050 & 0,139 & 0,205 & 0,106 & 198 & 0,280 & 154 \\
\hline & 0,0 & & & 174 & & 0,320 & 0,290 & 0,272 & & & 372 \\
\hline & 0,5 & 0,0 & & 0,256 & 0,248 & 1 & 0,416 & & & & 500 \\
\hline & 0,5 & $0, \sigma$ & & 310 & 0,357 & 0,606 & 0,487 & 0,558 & 719 & 580 & ,667 \\
\hline & \multicolumn{11}{|c|}{ Correlação AR(1) } \\
\hline \multirow[t]{4}{*}{0,0} & 0,0 & & & 01 & 0,041 & 0,146 & 0,198 & & & & 0,155 \\
\hline & 0,0 & 0,0 & 149 & 47 & & & 0,258 & & & &, 339 \\
\hline & 0,5 & 0,0 & 0,233 & 0,202 & 0,188 & 0,404 & 0,342 & &, 512 & & 429 \\
\hline & 0,5 & 0,5 & & 246 & 0,274 & 0,508 & 0,396 & 0,455 & 0,630 & 0,493 & 0,579 \\
\hline \multirow[t]{4}{*}{0,1} & 0,0 & & 057 & 0,096 & 0,044 & 0,140 & 0,190 & 0,101 & 0,194 & 253 & 0,158 \\
\hline & 0,0 & 0 & 168 & 154 & 0,1 & 0,301 & 0,271 & & & & \\
\hline & 0,5 & 0,0 & 254 & 0,216 & & & 0,357 & 0,365 & & & 462 \\
\hline & 0,5 & 0,5 & 347 & 268 & 0,315 & 0,536 & 0,418 & 0,485 & 0,660 & 517 & 0,602 \\
\hline \multirow[t]{4}{*}{0,3} & 0,0 & & & & 0,0 & 0,152 & 0,200 & 0,118 & 0,222 & 0,269 & 0,173 \\
\hline & 0,0 & & 27 & & 0,19 & 0,364 & 0,316 & & & & 417 \\
\hline & 0,5 & 0,0 & 329 & & & & & & & & 0,548 \\
\hline & 0,5 & 0,5 & 0,465 & & 0,431 & 0,652 & 0,545 & 0,598 & 744 & 616 & 0,715 \\
\hline \multirow[t]{4}{*}{0,7} & 0,0 & & & & 0,044 & & 0,199 & 0,10 & 10 & 0,273 & 0,161 \\
\hline & 0 , & & & & & 0,328 & 0,288 & & & & 0,381 \\
\hline & 0 & & & & & & & & & & 0,464 \\
\hline & 0,5 & & 0,368 & 0,290 & 0,329 & 0,560 & 0,447 & 0,511 & 0,672 & 0,546 & 0,625 \\
\hline
\end{tabular}


Tabela 5.4.8: Gama com regressores: poder das estatísticas $\xi_{k p}, \xi_{w}^{*}$ e $\xi_{w}^{+}$.

\begin{tabular}{|c|c|c|c|c|c|c|c|c|c|c|c|}
\hline \multicolumn{3}{|c|}{$n=50$} & \multicolumn{3}{|c|}{$\alpha=0,01$} & \multicolumn{3}{|c|}{$\alpha=0,05$} & \multicolumn{3}{|c|}{$\alpha=0,10$} \\
\hline$\rho$ & $\delta_{1}$ & $\delta_{2}$ & $\xi_{k p}$ & $\xi_{w}^{*}$ & $\xi_{w}^{+}$ & $\xi_{k p}$ & $\xi_{w}^{*}$ & $\xi_{w}^{+}$ & $\xi_{k p}$ & $\xi_{w}^{*}$ & $\xi_{w}^{+}$ \\
\hline \multicolumn{12}{|c|}{ Correlação simétrica } \\
\hline \multirow[t]{4}{*}{0,0} & 0,0 & 0,0 & 0,021 & 0,042 & 0,016 & 0,088 & 0.102 & 0,069 & 0,142 & 0,159 & 0,118 \\
\hline & 0.0 & 0,5 & 0,220 & 0,170 & 0,191 & 0,439 & 0.343 & 0,383 & 0,552 & 0,441 & 0,507 \\
\hline & 0,5 & 0,0 & 0,304 & 0,228 & 0,269 & 0,526 & 0.413 & 0,470 & 0,645 & 0,523 & 0,602 \\
\hline & 0.5 & 0,5 & 0,543 & 0,417 & 0,492 & 0,755 & 0.620 & 0,722 & 0,824 & 0,720 & 0,800 \\
\hline \multirow[t]{4}{*}{0,1} & 0,0 & 0,0 & 0,013 & 0,023 & 0,010 & 0,078 & 0.083 & 0,061 & 0,148 & 0,157 & 0,117 \\
\hline & 0,0 & 0,5 & 0,259 & 0,199 & 0,229 & 0,491 & 0.380 & 0,437 & 0,638 & 0,506 & 0,580 \\
\hline & 0.5 & 0,0 & 0,334 & 0,259 & 0,307 & 0,551 & 0.458 & 0,505 & 0,662 & 0,546 & 0,634 \\
\hline & 0.5 & 0,5 & 0,598 & 0,490 & 0,564 & 0.803 & 0.679 & 0,766 & 0,870 & 0,769 & 0,854 \\
\hline \multirow[t]{4}{*}{0,3} & 0.0 & 0,0 & 0,020 & 0,030 & 0,015 & 0,071 & 0.094 & 0,052 & 0,131 & 0,155 & 0,096 \\
\hline & 0,0 & 0,5 & 0,374 & 0,287 & 0,328 & 0,601 & 0.491 & 0,550 & 0,729 & 0,609 & 0,692 \\
\hline & 0,5 & 0,0 & 0,451 & 0,374 & 0,412 & 0,672 & 0.560 & 0,627 & 0,776 & 0,672 & 0,740 \\
\hline & 0,5 & 0,5 & 0,742 & 0,642 & 0,704 & 0,889 & 0.804 & 0,870 & 0,938 & 0,871 & 0,925 \\
\hline \multirow[t]{4}{*}{0,7} & 0,0 & 0,0 & 0,023 & 0,039 & 0,021 & 0,074 & 0,110 & 0,056 & 0,136 & 0,171 & 0,108 \\
\hline & 0,0 & 0,5 & 0,353 & 0,290 & 0,307 & 0,582 & 0,475 & 0,532 & 0,683 & 0,577 & 0,650 \\
\hline & 0,5 & 0,0 & 0,465 & 0,365 & 0,411 & 0,665 & 0.577 & 0,632 & 0,775 & 0,689 & 0,738 \\
\hline & 0,5 & 0,5 & 0,744 & 0,631 & 0,706 & 0,884 & 0.793 & 0,858 & 0,938 & 0,861 & 0,922 \\
\hline \multicolumn{12}{|c|}{ Correlação AR(1) } \\
\hline \multirow[t]{4}{*}{0,0} & 0,0 & 0,0 & 0,022 & 0,041 & 0,016 & 0,092 & 0,100 & 0,069 & 0,144 & 0,159 & 0,121 \\
\hline & 0,0 & 0,5 & 0,222 & 0,171 & 0,189 & 0,435 & 0,341 & 0,390 & 0,554 & 0,453 & 0,508 \\
\hline & 0,5 & 0,0 & 0,306 & 0,235 & 0,271 & 0,526 & 0,418 & 0,467 & 0,645 & 0,520 & 0,601 \\
\hline & 0.5 & 0,5 & 0,544 & 0,411 & 0,493 & 0,754 & 0.622 & 0,723 & 0,827 & 0,730 & 0,802 \\
\hline \multirow[t]{4}{*}{0,1} & 0,0 & 0,0 & 0,019 & 0,031 & 0,013 & 0,074 & 0,085 & 0,061 & 0,126 & 0,154 & 0,108 \\
\hline & 0,0 & 0,5 & 0,259 & 0,201 & 0,224 & 0,478 & 0,368 & 0,430 & 0,606 & 0,481 & 0,546 \\
\hline & 0,5 & 0,0 & 0,332 & 0,256 & 0,291 & 0,561 & 0,466 & 0,513 & 0,669 & 0,569 & 0,613 \\
\hline & 0,5 & 0,5 & 0,602 & 0,477 & 0,563 & 0,808 & 0.684 & 0,783 & 0,883 & 0,786 & 0,862 \\
\hline \multirow[t]{4}{*}{0,3} & 0,0 & 0,0 & 0,022 & 0,031 & 0,018 & 0,076 & 0,092 & 0,060 & 0,133 & 0,150 & 0,112 \\
\hline & 0,0 & 0,5 & 0,387 & 0,297 & 0,340 & 0,605 & 0,510 & 0,561 & 0,710 & 0,609 & 0,675 \\
\hline & 0,5 & 0,0 & 0,497 & 0,405 & 0,451 & 0,711 & 0,619 & 0,671 & 0,804 & 0,724 & 0,749 \\
\hline & 0,5 & 0,5 & 0,784 & 0,666 & 0,744 & 0,920 & 0,834 & 0,905 & 0,958 & 0,906 & 0,948 \\
\hline \multirow[t]{4}{*}{0,7} & 0,0 & 0,0 & 0,026 & 0,030 & 0,022 & 0,062 & 0,085 & 0,050 & 0,117 & 0,146 & 0,092 \\
\hline & 0,0 & 0,5 & 0,250 & 0,197 & 0,223 & 0,462 & 0,380 & 0,420 & 0,582 & 0,483 & 0,551 \\
\hline & 0,5 & 0,0 & 0,337 & 0,269 & 0,300 & 0,567 & 0,458 & 0,515 & 0,679 & 0,565 & 0,640 \\
\hline & 0,5 & 0,5 & 0,605 & 0,467 & 0,566 & 0,801 & 0,685 & 0,766 & 0,878 & 0,771 & 0,854 \\
\hline
\end{tabular}


Tabela 5.4.9: Gama com regressores: poder das estatísticas $\xi_{k p}, \xi_{w}^{*}$ e $\xi_{w}^{+}$.

\begin{tabular}{|c|c|c|c|c|c|c|c|c|c|c|c|}
\hline \multicolumn{3}{|c|}{$n=100$} & \multicolumn{3}{|c|}{$\alpha=0,01$} & \multicolumn{3}{|c|}{$\alpha=0,05$} & \multicolumn{3}{|c|}{$\alpha=0,10$} \\
\hline$\rho$ & $\delta_{1}$ & $\delta_{2}$ & $\xi_{k p}$ & $\xi_{w}^{*}$ & $\xi_{w}^{+}$ & $\xi_{k p}$ & $\xi_{w}^{*}$ & $\xi_{w}^{+}$ & $\xi_{k p}$ & $\xi_{w}^{*}$ & $\xi_{w}^{+}$ \\
\hline \multicolumn{12}{|c|}{ Correlação simétrica } \\
\hline \multirow[t]{4}{*}{0,0} & 0,0 & 0,0 & 0,014 & 0,012 & 0,012 & 0,067 & 0,073 & 0,051 & 0,119 & 0,130 & 0,102 \\
\hline & 0.0 & 0,5 & 0 & 0,319 & 0,350 & 0,624 & 0.522 & 0,582 & 0,743 & 0,642 & 0,711 \\
\hline & 0,5 & 0,0 & 0,438 & 0,353 & 0,402 & 0,673 & 0,563 & 0,628 & 0,780 & 0,674 & 0,755 \\
\hline & 0,5 & 0,5 & 0,728 & 0,618 & 0,694 & 0,884 & 0.789 & 0,861 & 941 & 861 & 0,925 \\
\hline \multirow[t]{4}{*}{0,1} & 0,0 & 0,0 & 0,019 & 0,014 & 0,015 & 0,066 & 0,068 & 0,057 & 0,117 & 0,114 & 0,096 \\
\hline & 0,0 & 0,5 & 80 & 0,401 & 0,437 & 0,701 & 0,608 & 0,660 & 807 & 0,709 & 0,777 \\
\hline & 0,5 & 0,0 & 0,507 & 0,413 & 0,457 & 0,719 & 0.621 & 0,677 & 0,837 & 0,723 & 0,802 \\
\hline & 0.5 & 0,5 & 0,808 & 0,710 & 0,779 & 0,933 & 0.872 & 0,918 & 0,975 & 0,919 & 0,963 \\
\hline \multirow[t]{4}{*}{0,3} & 0.0 & 0,0 & 0,019 & 0,017 & 0,016 & 0,069 & 0.068 & 0,059 & 0,118 & 0,126 & 0,105 \\
\hline & 0,0 & 0,5 & 643 & 0,565 & 0,602 & 0,834 & 0,755 & 0,803 & & & 0,884 \\
\hline & 0,5 & 0,0 & 0,6 & 0,574 & 0,618 & 0,860 & 0,783 & 0,837 & 0,921 & 0,858 & 0,905 \\
\hline & 0,5 & 0,5 & 923 & 0,866 & 0,905 & 0,983 & 0,956 & 0,978 & 0,995 & 0,978 & 0,993 \\
\hline \multirow[t]{4}{*}{0,7} & 0,0 & 0,0 & 0,022 & 0,024 & 0,019 & 0,068 & 0,083 & 0,061 & 0,115 & 0,129 & 0,091 \\
\hline & 0,0 & 0,5 & 0,644 & 0,554 & 0,601 & 0,844 & 0,760 & 805 & & 844 & 889 \\
\hline & 0,5 & 0,0 & 0,677 & 0,588 & 0,647 & 0,840 & 0,779 & 0,813 & 0,905 & & ,892 \\
\hline & 0,5 & 0,5 & 0,927 & 0,878 & 0,909 & 0,974 & 0,954 &, 970 &, 990 & 970 & 0,986 \\
\hline \multicolumn{12}{|c|}{ Correlação AR(1) } \\
\hline \multirow[t]{4}{*}{0,0} & 0,0 & 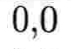 & 15 & 013 & 0,011 & 0,067 & 0,074 & 0,050 & & , 128 & 0,102 \\
\hline & 0,0 & 0,5 & 0,386 & 0,318 & & 622 & 0,523 & 0,583 & 0,7 & 0,643 & 0,713 \\
\hline & 0,5 & 0,0 & & 0,352 & 0,402 & 0,673 & 0,565 & 0,630 & 0,781 & 0,680 & 0,749 \\
\hline & 0,5 & 0,5 & 0,726 & 0,619 & 0,689 & 0,884 & 0,783 & 0,859 & 0,941 & 0,859 & 0,926 \\
\hline \multirow[t]{4}{*}{0,1} & 0,0 & 0,0 & 0,016 & 0,017 & 0,012 & 0,063 & 0,063 & 0,050 & 0,116 & 0,121 & 0,102 \\
\hline & 0,0 & 0,5 & 509 & 0,415 & 0,472 & 0,740 & 0,648 & 705 & &, 744 & 809 \\
\hline & 0,5 & 0,0 & 0,531 & 0,445 & 0,496 & 755 & 0,655 & 0,724 & 0,846 & 0,758 & 0,823 \\
\hline & 0,5 & 0,5 & 829 & 0,733 & 0,801 & 0,955 & 0,883 & 0,942 & 0,982 & 0,943 & 0,975 \\
\hline \multirow[t]{4}{*}{0,3} & 0,0 & 0,0 & 0,011 & 0,016 & 0,009 & 0,066 & 0,059 & 0,052 & 0,113 & 0,120 & 0,095 \\
\hline & 0,0 & 0,5 & 0,718 & 0,616 & 0,679 & 0,8 & 0,807 & 0,852 & 0,922 &, 873 & ),908 \\
\hline & 0,5 & 0,0 & & 0,634 & 0,692 & 0,894 & 0,832 & 0,871 & 0,9 & 0,893 & 0,928 \\
\hline & 0,5 & 0,5 & 0,959 & 0,912 & 0,944 & 0,992 & 0,975 & 0,990 & 0,997 & 0,990 & 0,997 \\
\hline \multirow[t]{4}{*}{0,7} & 0,0 & 0,0 & 014 & 019 & 0,012 & 0,063 & 0,082 & 0,053 & 0,11 & 0,137 & 0,090 \\
\hline & 0,0 & 0,5 & 504 & 417 & 0,463 & 0,737 & 0,643 & 0,714 & & 0,755 & 0,803 \\
\hline & 0,5 & 0,0 & & 443 & 0,492 & 0,752 & 0,656 & 0,711 & 0,858 &, 756 & 0,835 \\
\hline & 0,5 & 0,5 & 0,842 & 0,751 & 0,811 & 0,949 & 0,893 & 0,938 & 0,979 & 0,938 & 0,972 \\
\hline
\end{tabular}




\section{Capítulo 6}

\section{Contribuições, conclusões e propostas para futuras pesquisas}

Após pouco mais de dois anos e meio de pesquisa sobre testes para hipóteses restritas, reunimos nesta tese alguns resultados que apontam para as seguintes contribuições:

- Sumarizamos no Capítulo 2 os resultados de vários artigos relacionados com testes unilaterais multivariados para hipóteses restritas no modelo normal linear. Unificamos a notação e apresentamos os resultados sobre estimação sob restrição do vetor de parâmetros do modelo. Para os casos em que a matriz de covariância é conhecida ou desconhecida, apresentamos a relação entre as estatísticas da razão de verossimilhança, Kuhn-Tuker e Wald, bem como a distribuição nula assintótica dessas estatísticas;

- No Capítulo 3, apresentamos uma combinação do uso da estatística $\xi_{k p}$, proposta por Kodde e Palm (1986), com as Equações de Estimação Generalizadas (Liang e Zeger, 1986) juntamente com os resultados de Wolak (1991) para obter um razoável ganho de poder nos testes para hipóteses restritas; 
- Investigamos, no Capítulo 4, o poder de testes unilaterais na presença de dados correlacionados usando as Equações de Estimação Generalizadas. Acreditamos ser esta uma razoável contribuição uma vez que na literatura estatística encontramos apenas um trabalho sobre este assunto (Park et al., 1998), tratando somente do caso de comparação de grupos enquanto esta tese apresenta um estudo de poder dos testes para hipóteses restritas em modelos envolvendo regressores;

- Outra importante contribuição é a aplicação do teste de Follmann para hipóteses restritas usando Equações de Eestimação Generalizadas e envolvendo regressores, juntamente com um estudo de simulação comparando o poder do mesmo com os testes de Wald e Kodde e Palm.

Além dessas contribuições que acreditamos que foram dadas por esta tese, podemos tirar as seguintes conclusões:

- Como foi visto nos vários exemplos apresentados nesta tese, existem situações práticas em que um modelo de regressão com parâmetros restritos é mais adequado e essas restrições devem ser levadas em conta na análise dos dados;

- Através dos estudos de simulação, verificamos que há um razoável ganho de poder quando se usa a estatística de Kodde e Palm em relação a estatística de Wald, justamente pelo fato da estatística de Kodde e Palm levar em conta as restrições sobre os parâmetros do modelo sob análise;

- Verificamos também através dos vários estudos de simulação que a distribuição empírica da estatística de Kodde e Palm se aproxima bem da distribuição nula assintótica pelo menos para amostras de tamanho 100 . 
- A estatística de Kodde e Palm é fácil de ser aplicada em situações práticas, devendo a mesma ser preferida principalmente quando o número de restrições imposto pelas hipóteses é menor ou igual a 4. Quando o número de restrições é maior que 4, o cálculo do nível de significância do teste torna-se mais complicado e devemos recorrer a algoritmos específicos, o que torna a estatística de Kodde e Palm menos atrativa;

- Também através dos estudos de simulação, concluímos que o teste de Follmann apresenta um razoável ganho de poder em relação ao teste de Wald. Apesar do teste de Kodde e Palm apresentar um pequeno ganho de poder em relação ao teste de Follmann, este último é bastante simples de ser aplicado e evita o cálculo dos pesos exigidos pela estatística de Kodde e Palm. Isso torna o teste de Follmann um forte competidor principalmente quando o número de restrições imposto pelas hipóteses é maior que 4 .

Fica claro que sobre o tema "Testes para Hipóteses Restritas" ainda há bastante a ser explorado e para futuras pesquisas temos as seguintes propostas:

- Nos estudos sobre o poder de testes realizados nesta tese, os dados foram gerados segundo uma determinada estrutura de correlação e os estimadores EEGs foram obtidos levando-se em conta a mesma estrutura de correlação usada na geração dos dados. Nossa proposta é estudar o comportamento do poder dos testes de Wald, Kodde e Palm e Follmann quando a estrutura de correlação é incorreta, por exemplo, gerar dados com estrutura de correlação AR(1) e obter estimadores EEGs com estrutura de correlação simétrica ou não estruturada;

- Os estudos de simulação foram todos realizados considerando dados completos, seria interessante também, verificar o ganho de poder das estatísticas de Kodde e 
Palm e Follmann em relação a estatística de Wald quando há observações perdidas.

- Outra proposta. seria estender essa teoria para os parâmetros de correlação propondo por exemplo, testes para superdispersão. Neste caso, devemos estudar o problema de estimação usando Equações de Estimação Generalizadas para estimar conjuntamente os parâmetros do modelo e da matriz de correlação. 


\section{Apêndice A}

\section{Pesos}

\section{A.1 Introdução}

Os pesos que aparecem na distribuição nula das estatísticas dos testes para as hipóteses (2.4.1) e (2.4.2), dependem da matriz de covariância $\mathrm{V}$ e representam a probabilidade de que um vetor normal $k$-dimensional tenha exatamente $\ell$ componentes positivas, como foi visto no Teorema 2.2. Para esses pesos, existem soluções fechadas para o caso em que $k \leq 4$, cujas expressões podem ser encontradas, por exemplo, em Kudô (1963), Shapiro (1985) e Paula (1997). Para determinar as expressões para os pesos, vamos usar alguns conceitos apresentados a seguir e para mais detalhes, veja por exemplo, Shapiro $(1985,1988)$ e Perlman (1969). 


\section{A.2 Definições}

Um subconjunto não vazio $\mathcal{C} \in \mathbb{R}^{k}$ é chamado um cone (ou um conjunto positivamente homogêneo) se

$$
x \in \mathcal{C} \Rightarrow \lambda x \in \mathcal{C}, \quad \forall \lambda \in \mathbb{R}_{+}
$$

Vamos assumir que $\mathcal{C}$ é um cone convexo e fechado, o qual possui muitas propriedades úteis de espaços lineares. Seja $\mathrm{V}$ uma matriz $(k \times k)$ simétrica e positiva definida. A projeção ortogonal $\pi(x ; \mathrm{V}, \mathcal{C})$ de $x$ sobre $\mathcal{C}$, na métrica de $\mathrm{V}$, é qualquer ponto em $\mathcal{C}$ satisfazendo

$$
\min _{\boldsymbol{\mu}}\left\{(\boldsymbol{x}-\boldsymbol{\mu})^{T} \mathrm{~V}^{-1}(\boldsymbol{x}-\boldsymbol{\mu})\right\}, \boldsymbol{\mu} \in \mathcal{C} .
$$

Ao cone $\mathcal{C}$ está associado o cone polar $($ dual $) \mathcal{C}^{0}$, o qual é dado por

$$
\mathcal{C}^{0}=\left\{\boldsymbol{y}: \boldsymbol{x}^{T} \mathrm{~V}^{-1} \boldsymbol{y} \leq 0, \forall \boldsymbol{x} \in \mathcal{C}\right\}
$$

Como $\mathcal{C}$ é um cone convexo e fechado, então $\left(\mathcal{C}^{0}\right)^{0}=\mathcal{C}$ e vamos denotar por $\mathcal{F}(\mathcal{C})$ o conjunto de todas as faces de $\mathcal{C}$. A cada face $\phi \in \mathcal{F}(\mathcal{C})$ corresponde uma face $\phi^{*} \in \mathcal{F}\left(\mathcal{C}^{0}\right)$ tal que os espaços gerados por $\phi$ e $\phi^{*}$ são complementos ortogonais um do outro. Sejam $M_{\phi}$ e $M_{\phi^{*}}$ as matrizes de projeção ortogonal sobre os espaços gerados por $\phi$ e $\phi^{*}$, respectivamente. Note que $M_{\phi^{*}}=\mathrm{I}-M_{\phi}$, onde I é a matriz identidade. Vamos definir também

$$
\mathcal{S}_{\phi}=\left\{\boldsymbol{x} \in \mathbb{R}^{k}: \pi(\boldsymbol{x} ; \mathrm{V}, \mathcal{C}) \in \phi\right\} .
$$

Temos que, $\pi(x ; \mathrm{V}, \mathcal{C}) \in \phi$ se, e somente se, $M_{\phi} x \in \mathcal{C}$ e $M_{\phi^{*}} x \in \mathcal{C}^{0}$. Como $\left(\mathcal{C}^{0}\right)^{0}=\mathcal{C}$, então o conjunto $\mathcal{S}_{\phi}$ é definido pelas seguintes desigualdades:

$$
\left\{\begin{array}{l}
y^{T} M_{\phi} x \leq 0, \quad y \in \mathcal{C}^{0} \\
z^{T} M_{\phi^{*}} x \leq 0, \quad z \in \mathcal{C} .
\end{array}\right.
$$

Vamos determinar os pesos $w(k, \ell ; \mathbf{V})$ 's para o caso em que $\mathbf{V}=\mathbf{I}$, o caso geral pode ser reduzido a esse caso por transformação linear. 


\section{A.3 Expressões para os pesos}

Temos que $w(k, \ell ; \mathbf{I})$ é a probabilidade de $\pi(x ; \mathbf{I}, \mathcal{C})$ pertencer a uma face $\ell$-dimensional de $\mathcal{C}$, isto é,

$$
w(k, \ell ; \mathbf{I})=\sum P\left(x \in \mathcal{S}_{\phi}\right)
$$

onde a soma é feita sobre $\phi \in \mathcal{F}(\mathcal{C}) \operatorname{com} \operatorname{dim}(\phi)=\ell$. Das desigualdades (A.2.1) temos que

$$
P\left(\boldsymbol{x} \in \mathcal{S}_{\phi}\right)=P\left(\boldsymbol{x} \in W_{\phi}\right) P\left(\boldsymbol{x} \in W_{\phi^{*}}\right),
$$

onde $W_{\phi}=\left\{x: M_{\phi} x \in \phi\right\}$ e $W_{\phi^{*}}=\left\{y: M_{\phi^{*}} y \in \phi^{*}\right\}$.

Para $\phi=\{0\}$, o conjunto $W_{\phi}$ é todo o espaço $\mathbb{R}^{k}$ e portanto $P\left(x \in W_{\phi}\right)=1$. A face polar de $\{0\}$ é $\mathcal{C}^{0} \operatorname{logo}$

$$
w(k, k ; \mathbf{I})=P\left(\boldsymbol{x} \in \mathcal{C}^{0}\right) .
$$

Para uma face $\phi$ de dimensão 1 , o conjunto $W_{\phi}$ é a metade do espaço e portanto $P\left(x \in W_{\phi}\right)=1 / 2$, assim tem-se que

$$
w(k, k-1 ; \mathbf{I})=\frac{1}{2} \sum P\left(\boldsymbol{x} \in W_{\phi^{*}}\right),
$$

onde a soma é feita sobre todas as faces $\phi^{*} \in \mathcal{C}^{0} \operatorname{com} \operatorname{dim}\left(\phi^{*}\right)=k-1$. Para uma face $\phi$ de dimensão $2, P\left(\boldsymbol{x} \in W_{\phi}\right)=\frac{1}{2} \frac{1}{\pi} \theta(\phi)$, onde $\theta(\phi)$ é o ângulo plano de $\phi$. Então,

$$
w(k, k-2 ; \mathbf{I})=\frac{1}{2} \frac{1}{\pi} \sum \theta(\phi) P\left(x \in W_{\phi^{*}}\right),
$$

onde a soma é feita sobre todas as faces $\phi^{*}$ de $\mathcal{C}^{0} \operatorname{com} \operatorname{dim}\left(\phi^{*}\right)=k-2$, ou equivalentemente sobre todas as faces $\phi \in \mathcal{C} \operatorname{com} \operatorname{dim}(\phi)=2$. E assim por diante até

$$
w(k, 0 ; \mathbf{I})=P(\boldsymbol{x} \in \mathcal{C})
$$

Podemos observar que existe uma certa simetria entre os pesos correspondentes aos cones polares. Note que o conjunto $\mathcal{S}_{\phi}$ com respeito ao cone $\mathcal{C}$ é o mesmo conjunto 
como $\mathcal{S}_{\phi}$. com respeito ao cone $\mathcal{C}^{0}$, de modo que

$$
w(k, k-\ell ; \mathbf{I}, \mathcal{C})=w\left(k, \ell ; \mathbf{I}, \mathcal{C}^{0}\right), \quad \ell=0,1, \ldots, k
$$

Frequentemente o cone $\mathcal{C}$ é dado pelo quadrante generalizado não negativo $\mathbb{R}_{+}^{k}=\{\boldsymbol{x}$ : $\boldsymbol{x} \geq 0\}$ ou pode ser reduzido a esse caso por uma transformação linear, enquanto a matriz V é arbitrária. Para o cone $\mathcal{C}=\mathbb{R}_{+}^{k}$ os pesos $w(k, \ell ; \mathrm{V})$ 's podem ser calculados de forma fechada para $k \leq 4$ o que é suficiente para muitas situações práticas e serão apresentados a seguir.

Para $k=1$, temos que $\mathcal{C}=\{x \in \mathbb{R}: x \geq 0\}$ e

$$
w(1,0 ; \mathbf{V})=P(x \in \mathcal{C})=1 / 2=w(1,1 ; \mathbf{V})
$$

pois os pesos satisfazem $w(1,0 ; \mathbf{V})+w(1,1 ; \mathbf{V})=1$.

Seja $\rho_{i j}$ o coeficiente de correlação associado com a matriz V. Para $k=2$ e $k=3$, de acordo com os resultados de Shapiro (1985) os pesos ficam dados por

$$
\begin{gathered}
w(2,0 ; \mathbf{V})=\frac{1}{2 \pi}\left\{\pi-\cos ^{-1}\left(\rho_{12}\right)\right\} \\
w(2,1 ; \mathbf{V})=\frac{1}{2} \text { e } w(2,2 ; \mathbf{V})=\frac{1}{2 \pi} \cos ^{-1}\left(\rho_{12}\right) \\
w(3,0 ; \mathbf{V})=\frac{1}{4 \pi}\left\{2 \pi-\cos ^{-1}\left(\rho_{12}\right)-\cos ^{-1}\left(\rho_{13}\right)-\cos ^{-1}\left(\rho_{23}\right)\right\} ; \\
w(3,1 ; \mathbf{V})=\frac{1}{4 \pi}\left\{3 \pi-\cos ^{-1}\left(\rho_{12.3}\right)-\cos ^{-1}\left(\rho_{13.2}\right)-\cos ^{-1}\left(\rho_{23.1}\right)\right\} \\
w(3,2 ; \mathbf{V})=\frac{1}{2}-w(3,0 ; \mathbf{V}) \text { e } w(3,3 ; \mathbf{V})=\frac{1}{2}-w(3,1 ; \mathbf{V}),
\end{gathered}
$$

onde $\rho_{i j . k}$ é o coeficiente de correlação parcial de $x_{i}$ e $x_{j}$ fixando $x_{k}$ e é dado por

$$
\rho_{i j . k}=\frac{\rho_{i j}-\rho_{i k} \rho_{j k}}{\sqrt{\left(1-\rho_{i k}^{2}\right)\left(1-\rho_{j k}^{2}\right)}} .
$$

Para $k=4$, o peso $w(4,0 ; \mathrm{V})$ é a probabilidade de que todas as coordenadas de um $k$-vetor normal $\boldsymbol{x}$ sejam não negativas. Não há um método simples para calcular essa 
probabilidade, ela pode ser obtida por integração numérica da função de distribuição da normal multivariada. Paula (1997), por exemplo, apresenta uma fórmula para calcular essa probabilidade. Para $k \geq 4$ essa probabilidade pode ser calculada usando-se a fórmula geral proposta por Kendall (1941), a qual foi aplicada por Kudô (1963). Os outros pesos para $k=4$ são dados por

$$
\begin{aligned}
& w(4,1 ; \mathrm{V})=\frac{1}{8 \pi}\left\{8 \pi-\sum_{i>j: i, j \neq k} \cos ^{-1}\left(\rho_{i j . k}\right)\right\} ; \\
& w(4,2 ; \mathrm{V})=\frac{1}{4 \pi^{2}}\left\{\sum_{i>j, k>\ell: k, \ell \neq i, j} \cos ^{-1}\left(\rho_{i j}\right)\left[\pi-\cos ^{-1}\left(\rho_{k \ell . i j}\right)\right]\right\} ; \\
& w(4,3 ; \mathrm{V})=\frac{1}{8 \pi}\left\{-4 \pi+\sum_{i>j: i, j \neq k} \cos ^{-1}\left(\rho_{i j . k}\right)\right\} \mathrm{e} \\
& w(4,4 ; \mathrm{V})=\frac{1}{2}-w(4,0 ; \mathrm{V})-w(4,2 ; \mathrm{V}),
\end{aligned}
$$

onde $\rho_{k \ell . i j}$ é o coeficiente de correlação parcial enter $x_{k}$ e $x_{\ell}$ fixando $x_{i}$ e $x_{j}$ e é dado por

$$
\rho_{k \ell . i j}=\frac{\rho_{k \ell . i}-\rho_{k j . i} \rho_{\ell j . i}}{\sqrt{\left(1-\rho_{k j . i}^{2}\right)\left(1-\rho_{\ell j . i}^{2}\right)}} .
$$

No caso particular em que a matriz de covariância é dada por $\mathrm{V}=\sigma^{2} \mathbf{I}$, os pesos existem na forma fechada para todo $k$ e são dados por

$$
w\left(k, \ell ; \sigma^{2} \mathbf{I}\right)=\frac{1}{2^{k}}\left(\begin{array}{c}
k \\
\ell
\end{array}\right)=\frac{1}{2^{k}}\left(\begin{array}{c}
k \\
k-\ell
\end{array}\right)=w\left(k, k-\ell ; \sigma^{2} \mathbf{I}\right)
$$

(veja, por exemplo, Wolak, 1987).

Uma fórmula reduzida para $w(k, \ell ; \mathrm{V})$ com $k$ arbitrário é dada em Childs (1967), bem como uma metodologia particular para a simplificação de $w(k, \ell ; \mathrm{V})$ em uma única integral no intervalo $[0,1]$ para o caso de $k=4$. Sun (1988a) estende essa metodologia para $k \geq 4$, mostrando que a probabilidade do quadrante positivo generalizado pode ser reduzida a uma combinação linear de integrais de ordem $([k / 2]-1)$ no intervalo $[0,1]$. Assim, para $k=4$ e $k=5$, é preciso desenvolver apenas uma única integral para obter $w(k, \ell ; \mathrm{V})$. Já, para os casos de $k=6$ e $k=7$, é necessário uma integração 
dupla no conjunto $[0,1] \times[0,1]$, e assim sucessivamente. Um programa em Fortran foi desenvolvido por Bohrer e Chow (1978) para o cálculo dessas probabilidades para $k \leq 10$. Sun (1988b) também desenvolveu um programa em Fortran para o cálculo de $w(k, \ell ; \mathbf{V})$.

As dificuldades no cálculo da probabilidade do quadrante positivo generalizado de uma normal multivariada tem motivado o desenvolvimento de vários estudos no sentido de obter aproximações para os pesos $w(k, \ell ; \mathrm{V})$ 's. Robertson et al. (1988, Capítulo 3), apresentam uma excelente revisão de procedimentos desenvolvidos para simplificar o cálculo de $w(k, \ell ; \mathrm{V})$, quando o interesse é testar hipóteses de quase-ordem ${ }^{1}$ para as médias de $k$ normais independentes. Nesses casos, a matriz $\mathrm{V}$ toma formas especiais que facilitam a simplificação dos pesos.

Uma metodologia para calcular esses pesos para os casos em que $k \geq 8$, é usar a técnica de Monte Carlo: gera-se uma amostra de tamanho 1000, por exemplo, de uma distribuição normal multivariada com vetor de médias zero e matriz de covariância $\mathrm{V}$. Para cada elemento $x$ da amostra, calcula-se $\pi(x ; \mathrm{V}, \mathcal{C})=\tilde{\boldsymbol{x}}$ e conta-se o número de componentes de $\tilde{\boldsymbol{x}}$ maiores que zero. O valor atribuido a $w(k, \ell ; \mathrm{V})$ é a proporção de $\tilde{\boldsymbol{x}}$ 's com exatamente $\ell$ componentes maiores que zero. Esse procedimento tem a vantagem de não requerer técnicas de integração numérica despendiosas e não limita o valor de $k$. Entretanto, os pesos não são exatos.

\footnotetext{
${ }^{1}$ se $\mu_{i} \leq \mu_{j}$ e $\mu_{j} \leq \mu_{k}$ então $\mu_{i} \leq \mu_{k}$
} 


\section{Apêndice B}

\section{Geração de variáveis correlacionadas}

Será descrito a seguir os procedimantos usados para gerar variáveis aleatórias correlacionadas. O procedimento proposto por Park et al. (1996) para gerar variáveis binárias correlacionadas o o procedimento proposto por Park e Shin (1998) para gerar variáveis correlacionadas com distribuição de Poisson e distribuição gama.

\section{B.1 Variáveis binárias}

Dados $\mu_{1}, \mu_{2}, \ldots, \mu_{k}$ e $\rho_{i j} \geq 0$, para $1 \leq i, j \leq k$, o procedimento gera um vetor $\mathbf{Z}=$ $\left(Z_{1}, Z_{2}, \ldots, Z_{k}\right)^{T}$ de variáveis binárias tal que $\mathrm{E}\left(Z_{i}\right)=\mu_{i}$ e $\operatorname{Corr}\left(Z_{i}, Z_{j}\right)=\rho_{i j}, \forall i \neq j$. Para apresentar a idéia vamos considerar $k=2$ e denotar $X(\alpha)$ uma variável aleatória com distribuição de Poisson de média $\alpha \geq 0$. Por convenção $X(0)=0$. As variáveis $X$ 's com índices diferentes são consideradas mutuamente independentes. Sejam $Y_{1}$ e $Y_{2}$ 
variáveis aleatórias definidas por

$$
\begin{aligned}
& Y_{1}=X_{1}\left(\alpha_{1}-\alpha_{12}\right)+X_{3}\left(\alpha_{12}\right) \mathrm{e} \\
& Y_{2}=X_{2}\left(\alpha_{2}-\alpha_{12}\right)+X_{3}\left(\alpha_{12}\right),
\end{aligned}
$$

onde $\alpha_{1}, \alpha_{2}$ e $\alpha_{12}$ são constantes não negativas. Como as variáveis $X$ 's são independentes, então $Y_{1}$ e $Y_{2}$ têm distribuição de Poisson com médias $\alpha_{1}$ e $\alpha_{2}$, respectivamente, e são não negativamente correlacionadas por causa do termo comum $X_{3}\left(\alpha_{12}\right)$. Definindo

$$
Z_{1}=I_{\{0\}}\left(Y_{1}\right) \quad \text { e } \quad Z_{2}=I_{\{0\}}\left(Y_{2}\right)
$$

onde $I_{\{\cdot\}}$ é a função indicadora, tem-se que $Z_{1}$ e $Z_{2}$ são não negativamente correlacionadas. As constantes $\alpha_{1}, \alpha_{2}$ e $\alpha_{12}$ podem ser escolhidas de modo que $\mathrm{E}\left(Z_{1}\right)=\mu_{1}, \mathrm{E}\left(Z_{2}\right)=$ $\mu_{2}$ e $\operatorname{Corr}\left(Z_{1}, Z_{2}\right)=\rho_{12}$, como segue: para $i=1,2$

$$
\mathrm{E}\left(Z_{i}\right)=\mathrm{E}\left(Z_{i}^{2}\right)=P\left(Y_{i}=0\right)=P\left(X_{i}=X_{3}=0\right)=e^{-\alpha_{i}}
$$

e portanto devemos escolher $\alpha_{i}=-\ln \left(\mu_{i}\right)$. Por outro lado, temos que

$$
\begin{gathered}
\operatorname{Var}\left(Z_{i}\right)=\mu_{i}-\mu_{i}^{2}=\mu_{i}\left(1-\mu_{i}\right) \\
\mathrm{E}\left(Z_{1} Z_{2}\right)=P\left(Y_{1}=Y_{2}=0\right)=P\left(X_{1}=X_{2}=X_{3}=0\right)=\mu_{1} \mu_{2} e^{\alpha_{12}} \\
\operatorname{Cov}\left(Z_{1}, Z_{2}\right)=\mu_{1} \mu_{2} e^{\alpha_{12}}-\mu_{1} \mu_{2}=\mu_{1} \mu_{2}\left(e^{\alpha_{12}}-1\right) \mathrm{e} \\
\rho_{12}=\mu_{1} \mu_{2}\left(e^{\alpha_{12}}-1\right)\left\{\mu_{1}\left(1-\mu_{1}\right) \mu_{2}\left(1-\mu_{2}\right)\right\}^{-1 / 2}
\end{gathered}
$$

Portanto, devemos escolher

$$
\alpha_{12}=\ln \left\{1+\rho_{12}\left[\frac{\left(1-\mu_{1}\right)\left(1-\mu_{2}\right)}{\mu_{1} \mu_{2}}\right]^{1 / 2}\right\} .
$$

$\mathrm{O}$ caso em que $\alpha_{12}=0$ corresponde a independência entre $Z_{1}$ e $Z_{2}$. Como $\mathrm{E}\left(Z_{1} Z_{2}\right)=$ $P\left(Z_{1}=Z_{2}=1\right) \leq P\left(Z_{i}=1\right)=\mu_{i}$, então $\operatorname{Cov}\left(Z_{1}, Z_{2}\right) \leq \mu_{1}\left(1-\mu_{2}\right)$ e $\operatorname{Cov}\left(Z_{1}, Z_{2}\right) \leq$ 
$\mu_{2}\left(1-\mu_{1}\right)$ e portanto $\rho_{12}$ não varia livremente no intervalo $[-1,1]$, o mesmo deve satisfazer as seguintes desigualdades:

$$
\rho_{12} \leq\left\{\frac{\mu_{1}\left(1-\mu_{2}\right)}{\mu_{2}\left(1-\mu_{1}\right)}\right\}^{1 / 2} \quad \text { e } \quad \rho_{12} \leq\left\{\frac{\mu_{2}\left(1-\mu_{1}\right)}{\mu_{1}\left(1-\mu_{2}\right)}\right\}^{1 / 2} .
$$

Essas desigualdades levam à relação $\alpha_{12} \leq \ln \left(\mu_{i}^{-1}\right)=\alpha_{i}$, portanto o procedimento gera variáveis binárias não negativamente correlacionadas.

Para o caso geral em que $k>2$, considera-se $k$ variáveis aleatórias $Y_{1}, Y_{2}, \ldots, Y_{k}$, com distribuição de Poisson que são somas parciais das variáveis aleatórias independentes com distribuição de Poisson $X_{1}\left(\beta_{1}\right), X_{2}\left(\beta_{2}\right), \ldots, X_{t}\left(\beta_{t}\right)$, para algum inteiro não negativo $t$ e números reais $\beta_{1}, \beta_{2}, \ldots, \beta_{t}$. Alguns dos $X$ 's podem aparecer simultaneamente em vários Y's. O valor esperado e a estrutura de correlação das variáveis binárias $Z_{1}=I_{\{0\}}\left(Y_{1}\right), Z_{2}=I_{\{0\}}\left(Y_{2}\right), \ldots, Z_{k}=I_{\{0\}}\left(Y_{k}\right)$ são obtidos controlando-se o padrão de somas parciais dos $X$ 's em cada $Y$ e os valores de $\beta_{1}, \beta_{2}, \ldots, \beta_{t}$. O algoritmo abaixo descreve como obter $t, \beta_{1}, \beta_{2}, \ldots, \beta_{t}$ e o padrão de somas parciais dados $\mathrm{E}\left(Z_{i}\right)=\mu_{i}$ e $\operatorname{Cov}\left(Z_{i}, Z_{j}\right)=\rho_{i j}, i \neq j, 1 \leq i, j \leq k$.

\section{Algoritmo:}

Passo 0. Faça $\ell=0$ e para $1 \leq i, j \leq k$ calcule

$$
\alpha_{i j}=\ln \left\{1+\rho_{i j}\left[\frac{\left(1-\mu_{i}\right)\left(1-\mu_{j}\right)}{\mu_{i} \mu_{j}}\right]^{1 / 2}\right\} .
$$

Passo 1. Faça $\ell=\ell+1$ e seja $T_{\ell}=\left\{\alpha_{i j}: \alpha_{i j}>0,1 \leq i, j \leq k\right\}$. Determine $\beta_{\ell}=\alpha_{r s}=\min \left\{\alpha_{i j}: \alpha_{i j} \in T_{\ell}\right\}$, isto é, $\beta_{\ell}$ é o menor elemento do conjunto $T_{\ell}$. Se $\alpha_{r r}=0$ ou $\alpha_{s s}=0$ então pare. Caso contrário, escolha um conjunto de índices $S_{\ell}$ contendo $\{r, s\}$ e satisfazendo $\alpha_{i j}>0 \forall i, j \in S_{\ell}$.

Passo 2. Atualize o conjunto $T_{\ell-1}$ substituindo $\alpha_{i j}$ por $\alpha_{i j}-\beta_{\ell} \forall i, j \in S_{\ell}$. Se todo $\alpha_{i j}=0$, então vá para o passo 3 . Caso contrário, volte ao passo 1 . 
Passo 3. Faça $t=\ell$. Para $i=1,2, \ldots, k$, seja

$$
Y_{i}=\sum_{\ell=1}^{t} X_{\ell}\left(\beta_{\ell}\right) I_{S_{\ell}}(i) \quad \text { e } \quad Z_{i}=I_{\{0\}}\left(Y_{i}\right)
$$

Observação 1: note que no passo 1 o menor $\beta_{\ell}$ é escolhido de modo que todas as variáveis Poisson tenham médias não negativas. É possível que $\alpha_{r s}$ e $S_{\ell}$ não sejam únicos no passo 1 . Nesse caso pode-se escolher $\alpha_{r s}$ e $S_{\ell}$ arbitrariamente.

Observação 2: no caso particular em que $\mu_{1}=\mu_{2}=\cdots=\mu_{k}=\mu$ e $\rho_{i j}=\rho, \forall i \neq j$, uma simplificação é possível fazendo-se $\alpha=\ln \left\{1+\rho(1-\mu) \mu^{-1}\right\}$ e $\beta=\ln \left(\mu^{-1}\right)$. Com isso tem-se que $t=k+1, \alpha_{i i}=\beta, \alpha_{i j}=\alpha \forall i \neq j, \beta_{1}=\alpha, \beta_{j}=\beta, j=2, \ldots, t$, os outros $\beta$ 's são todos iguais a zero e

$$
Y_{i}=X_{1}(\alpha)+X_{i+1}(\beta-\alpha), \quad i=1,2, \ldots, k
$$

Nesse caso são necessárias $t=k+1$ variáveis aleatórias Poisson independentes. No caso de estrutura $A R(1)$ todos os $\beta$ 's são diferentes de zero e são necesárias $t=k(k+1) / 2$ variáveis aleatórias Poisson independentes.

\section{B.2 Variáveis Poisson e gama}

O algoritmo usado para gerar variáveis aleatórias correlacionadas com distribuição de Poisson e distribuição gama, foi proposto por Park e Shin (1998) para gerar variáveis correlacionas cuja distribuição pertence a uma classe de distribuições fechadas para soma de acordo com a seguinte definição:

Definição B.1. Uma classe de funções de distribuição $\mathcal{S}=\left\{F_{\alpha}: \alpha>0\right\}$ é fechada para soma se $F_{\alpha} \in \mathcal{S}$ e $F_{\beta} \in \mathcal{S} \Longrightarrow F_{\alpha} * F_{\beta} \in \mathcal{S}$, onde $F_{\alpha} * F_{\beta}$ é a convolução de $F_{\alpha}$ e $F_{\beta}$ definida por $\left(F_{\alpha} * F_{\beta}\right)(x)=\int_{-\infty}^{\infty} F_{\alpha}(x-u) d F_{\beta}(u)$. 
O fato da classe $\mathcal{S}$ ser fechada para soma é equivalente ao fato de que, para quaisquer distribuições $F_{\alpha}, F_{\beta} \in \mathcal{S}$, a soma das variáveis aleatórias independentes com distribuição $F_{\alpha}$ e $F_{\beta}$ tem função de distribuição $F_{\alpha+\beta}$. Um exemplo típico é a classe de todas as distribuições de Poisson. Se $X_{1}(\alpha)$ e $X_{2}(\beta)$ são independentes com distribuição de Poisson com parâmetros $\alpha$ e $\beta$, respectivamente, então $X_{1}(\alpha)+X_{2}(\beta)$ tem distribuição de Poisson comparâmetro $\alpha+\beta$.

Dados o vetor de médias $\boldsymbol{\mu}=\left(\mu_{1}, \mu_{2}, \ldots, \mu_{k}\right)^{T}$, a matriz de correlação $\Gamma=\left(\rho_{i j}\right)$ e a classe de distribuições $\mathcal{S}$, para gerar o vetor $\boldsymbol{Y}=\left(Y_{1}, Y_{2}, \ldots, Y_{k}\right)^{T}$ de variáveis aleatórias não negativamente correlacionadas na classe $\mathcal{S}$ tal que $\mathrm{E}\left(Y_{i}\right)=\mu_{i}$ e $\operatorname{Corr}\left(Y_{i}, Y_{j}\right)=\rho_{i j} \geq$ $0 \forall i \neq j$, o algoritmo determina um inteiro $t$, números reais $\beta_{1}, \beta_{2}, \ldots, \beta_{t}$, uma matriz $\boldsymbol{T}$ de zeros e uns e um vetor $\boldsymbol{X}=\left(X_{1}\left(\beta_{1}\right), X_{2}\left(\beta_{2}\right), \ldots, X_{t}\left(\beta_{t}\right)\right)^{T}$ tal que o vetor aleatório $\boldsymbol{Y}=\boldsymbol{T} \boldsymbol{X}$ tem média $\boldsymbol{\mu}$ e matriz de correlação $\Gamma$.

\section{Algoritmo:}

Passo 1. Faça $\ell=1$ e para $1 \leq i, j \leq k$ calcule

$$
\alpha_{i j}=\rho_{i j}\left(\mu_{i} \mu_{j}\right)^{1 / 2}
$$

Seja $T_{\ell}=\left\{\alpha_{i j}: \alpha_{i j}>0,1 \leq i, j \leq k\right\}$.

Passo 2. Determine $\beta_{\ell}=\alpha_{r s}^{\ell}=\min \left\{\alpha_{i j}: \alpha_{i j} \in T_{\ell}\right\}$, isto é, $\beta_{\ell}$ é o menor elemento do conjunto $T_{\ell}$. Escolha um conjunto de índices $S_{\ell}$ contendo $\{r, s\}$ e satisfazendo $\alpha_{i j}^{\ell}>0 \forall i, j \in S_{\ell}$.

Passo 3. Atualize o conjunto $T_{\ell}$ fazendo

$$
\alpha_{i j}^{\ell+1}= \begin{cases}\alpha_{i j}^{\ell}-\beta_{\ell} & \forall i \in S_{\ell} \text { e } j \in S_{\ell} \\ \alpha_{i j}^{\ell} & \forall i \notin S_{\ell} \text { ou } j \notin S_{\ell} .\end{cases}
$$

Se todo $\alpha_{i j}^{\ell+1}=0$ vá para o passo 4 . Caso contrário, faça $\ell=\ell+1$ e volte aos passo 2 . 
Passo 4. Faça $t=\ell$. Construa a matriz $(k \times t) \boldsymbol{T}=\left[T_{i \ell}\right]$ com

$$
T_{i \ell}=\left\{\begin{array}{lll}
1 & \text { se } & i \in S_{\ell} \\
0 & \text { se } & i \notin S_{\ell}
\end{array}\right.
$$

para $i=1,2, \ldots, k$ e $\ell=1,2, \ldots, t$.

Observação 1: a distribuição de $\boldsymbol{Y}=\boldsymbol{T} \boldsymbol{X}$ depende da distribuição dos elementos do vetor $\boldsymbol{X}=\left(X_{1}\left(\beta_{1}\right), X_{2}\left(\beta_{2}\right), \ldots, X_{t}\left(\beta_{t}\right)\right)^{T}$. Se $X_{i}\left(\beta_{i}\right)$ tem distribuição de Poisson, então $\boldsymbol{Y}$ terá distribuição de Poisson. Se a distribuição de $X_{i}\left(\beta_{i}\right)$ é gama, então $\boldsymbol{Y}$ terá distribuição gama.

Observação 2: é possível que $\alpha_{r s}$ e $S_{\ell}$ não sejam únicos no passo 2. Nesse caso pode-se escolher o primeiro $\alpha_{r s}$ e $S_{\ell}$ encontrados ou considerar todos os conjuntos encontrados.

Observação 3: no caso particular em que $\mu_{1}=\mu_{2}=\cdots=\mu_{k}=\mu$ e $\rho_{i j}=\rho \forall i \neq j$, uma simplificação é possível fazendo-se $\alpha_{i i}=\mu$ e $\alpha_{i j}=\rho \mu \forall i \neq j$. Com isso tem-se que $t=k+1, \quad \beta_{1}=\rho \mu, \quad \beta_{\ell}=\mu-\rho \mu, \ell=2,3, \ldots, t \mathrm{e}$

$$
Y_{i}=X_{1}(\rho \mu)+X_{i+1}(\mu-\rho \mu), \quad i=1,2, \ldots, k .
$$

Observação 4: no caso particular em que a estrutura de correlação é um AR(1) com $\rho_{i j}=\rho^{|i-j|}$, se $\rho \leq \min \left\{\left(\mu_{j+1} / \mu_{j}\right)^{1 / 2}, j=1,2, \ldots, k-1\right\}$ então

$$
Y_{i}=\sum_{t=1}^{i} \sum_{s=1}^{k-i+1} X_{t s}\left(\beta_{t s}\right), \quad i=1,2, \ldots, k,
$$

onde $\beta_{11}=\alpha_{1 k}, \quad \beta_{1 s}=\alpha_{1, k-s+1}-\alpha_{1, k-s+2}, s=2, \ldots, k$,

$$
\begin{gathered}
\beta_{t 1}=\alpha_{t k}-\alpha_{t-1, k}, \beta_{t s}=\left(\alpha_{t, k-s+1}-\alpha_{t, k-s+2}\right)-\left(\alpha_{t-1, k-s+1}-\alpha_{t-1, k-s+2}\right), \\
s=2, \ldots, k-t+1, t=2, \ldots, k-1 \mathrm{e} \\
\beta_{k 1}=\alpha_{k k}-\alpha_{k-1, k} .
\end{gathered}
$$




\section{Apêndice C}

\section{Nota sobre o poder}

Vamos supor que $y_{1 j}$ e $y_{2 j}$ são variáveis aleatórias tais que $\mathrm{E}\left(y_{1 j}\right)=\mu_{1}, \mathrm{E}\left(y_{2 j}\right)=$ $\mu_{2}, \operatorname{Var}\left(y_{1 j}\right)=\sigma_{1}^{2}$ e $\operatorname{Var}\left(y_{2 j}\right)=\sigma_{2}^{2}, j=1,2, \ldots, m$, e que o interesse é testar

$$
H_{0}: \mu_{2}-\mu_{1}=0 \text { versus } H_{1}: \mu_{2}-\mu_{1} \neq 0 \text {. }
$$

Vamos considerar duas situações.

\section{C.1 Situação I}

Suponha que $\operatorname{Corr}\left(y_{1 j}, y_{1 j^{\prime}}\right)=\operatorname{Corr}\left(y_{2 j}, y_{2 j^{\prime}}\right)=\rho$, para $\rho \geq 0, j \neq j^{\prime}$ e $\operatorname{Corr}\left(y_{1 j}, y_{2 j^{\prime}}\right)=$ $0, \forall j, j^{\prime}$. Logo, teremos que $\operatorname{Cov}\left(y_{1 j}, y_{1 j^{\prime}}\right)=\rho \sigma_{1}^{2}$ e $\operatorname{Cov}\left(y_{2 j}, y_{2 j^{\prime}}\right)=\rho \sigma_{2}^{2}$, para $j \neq j^{\prime} . \mathrm{A}$ variância da diferença $\bar{y}_{2}-\bar{y}_{1}$, fica dada por

$$
\operatorname{Var}\left(\bar{y}_{2}-\bar{y}_{1}\right)=\operatorname{Var}\left(\bar{y}_{2}\right)+\operatorname{Var}\left(\bar{y}_{1}\right),
$$


onde

$$
\begin{aligned}
\operatorname{Var}\left(\bar{y}_{i}\right) & =\frac{1}{m^{2}} \operatorname{Var}\left(\sum_{j=1}^{m} y_{i j}\right) \\
& =\frac{1}{m^{2}}\left\{\sum_{j=1}^{m} \operatorname{Var}\left(y_{i j}\right)+m(m+1) \sum_{j \neq j^{\prime}} \operatorname{Cov}\left(y_{i j}, y_{i j^{\prime}}\right)\right\} \\
& =\frac{1}{m^{2}}\left\{m \sigma_{i}^{2}+m(m+1) \rho \sigma_{i}^{2}\right\} \\
& =\frac{\sigma_{i}^{2}}{m}\{1+(m+1) \rho\}, \quad i=1,2 .
\end{aligned}
$$

$\mathrm{O}$ teste $t$-pareado fica, então, dado por

$$
\begin{aligned}
t & =\frac{\bar{y}_{2}-\bar{y}_{1}}{\left\{\operatorname{Var}\left(\bar{y}_{2}-\bar{y}_{1}\right)\right\}^{1 / 2}} \\
t & =\frac{\left(\bar{y}_{2}-\bar{y}_{1}\right)}{\left\{\frac{1}{m}[1+(m+1) \rho]\left(\sigma_{1}^{2}+\sigma_{2}^{2}\right)\right\}^{1 / 2}}
\end{aligned}
$$

Para $\left(\bar{y}_{2}-\bar{y}_{1}\right)$ fixo o denominador em (C.1.1) cresce à medida que $\rho$ cresce. Isso significa que o poder do teste decresce com o aumento da correlação.

\section{C.2 Situação II}

Vamos supor agora que $\operatorname{Corr}\left(y_{1 j}, y_{2 j}\right)=\rho$, para $\rho \geq 0$ e $j=1,2, \ldots, m$ e que $\operatorname{Corr}\left(y_{i j}, y_{i j^{\prime}}\right)=0, \forall j \neq j^{\prime}$ e $i=1,2$. Logo teremos $\operatorname{Cov}\left(y_{1 j}, y_{2 j}\right)=\rho \sigma_{1} \sigma_{2}$. A variância da diferença $\bar{y}_{2}-\bar{y}_{1}$, fica dada por

$$
\operatorname{Var}\left(\bar{y}_{2}-\bar{y}_{1}\right)=\operatorname{Var}\left(\bar{y}_{2}\right)+\operatorname{Var}\left(\bar{y}_{1}\right)-2 \operatorname{Cov}\left(\bar{y}_{2}, \bar{y}_{1}\right)
$$

onde $\operatorname{Var}\left(\bar{y}_{i}\right)=\sigma_{i}^{2} / m, i=1,2 \mathrm{e}$

$$
\begin{aligned}
\operatorname{Cov}\left(\bar{y}_{2}, \bar{y}_{1}\right) & =\frac{1}{m^{2}} \sum_{j=1}^{m} \operatorname{Cov}\left(y_{1 j}, y_{2 j}\right) \\
& =\frac{1}{m^{2}} m \rho \sigma_{1} \sigma_{2}=\rho \sigma_{1} \sigma_{2} / m .
\end{aligned}
$$

$O$ teste $t$-pareado fica, neste caso, dado por

$$
t=\frac{\left(\bar{y}_{2}-\bar{y}_{1}\right)}{\left\{\frac{\sigma_{1}^{2}}{m}+\frac{\sigma_{2}^{2}}{m}-\frac{\rho \sigma_{1} \sigma_{2}}{m}\right\}^{1 / 2}} .
$$


Para $\left(\bar{y}_{2}-\bar{y}_{1}\right)$ fixo o denominador em (C.2.2) decresce à medida que $\rho$ cresce. Ou seja, o poder do teste cresce à medida que aumenta a correlação.

Suponha agora que temos uma situação um pouco diferente em que $\mathrm{E}\left(y_{i j}\right)=\boldsymbol{x}_{i}^{T} \beta$, $j=1,2, \operatorname{Var}\left(y_{i j}\right)=\sigma^{2}, \operatorname{Cov}\left(y_{i 1}, y_{i 2}\right)=\rho \sigma^{2}$ e as demais correlações sendo nulas. Logo, definindo $y_{i}=\left(y_{i 1}, y_{i 2}\right)^{T}$ temos que

$$
\operatorname{Var}\left(\boldsymbol{y}_{i}\right)=\mathbf{V}_{i}=\left[\begin{array}{cc}
\sigma^{2} & \rho \sigma^{2} \\
\rho \sigma^{2} & \sigma^{2}
\end{array}\right] .
$$

O estimador de mínimos quadrados de $\beta$ fica dado por

$$
\hat{\beta}=\sum_{i=1}^{n} \frac{\boldsymbol{x}_{i}^{T} \mathrm{~V}_{i} \boldsymbol{y}_{i}}{\boldsymbol{x}_{i}^{T} \mathrm{~V}_{i} \boldsymbol{x}_{i}}=\sum_{i=1}^{n} \frac{\bar{y}_{i}}{x_{i}}
$$

onde $\boldsymbol{x}_{i}=\left(x_{i}, x_{i}\right)^{T}$ e $\bar{y}_{i}=\left(y_{i 1}+y_{i 2}\right) / 2$. Obtemos facilmente

$$
\operatorname{Var}(\hat{\beta})=\sum_{i=1}^{n} \frac{\operatorname{Var}\left(\bar{y}_{i}\right)}{x_{i}^{2}}=\frac{n \sigma^{2}(2+\rho)}{4 \sum_{i=1}^{n} x_{i}^{2}} .
$$

Logo, para testar $H_{0}: \beta=\beta_{0}$ versus $H_{1}: \beta \neq \beta_{0}$ a estatística $t$ fica dada por

$$
t=\frac{\sum_{i=1}^{n} \frac{\bar{y}_{i}}{x_{i}}-\beta_{0}}{n \sigma^{2}(2+\rho) \sum_{i=1}^{n} x_{i}^{2}},
$$

que para $x_{i}$ e $\bar{y}_{i}$ fixos, $\forall i$, decresce à medida que $\rho$ cresce. Isso significa poder baixo para altos valores da correlação. 


\section{Apêndice D}

\section{Conjuntos de dados}

Apresentamos a seguir os conjuntos de dados que foram usados nos exemplos de aplicação dos resultados deste trabalho.

\section{D.1 Assinaturas de TV a cabo}

A Tabela D.1.1 apresenta os dados sobre a assinatura de TV a cabo. As variáveis nessa tabela são descritas a seguir:

Nass: número de assinaturas (em milhares) de TV a cabo;

Domic: número de domicílios (em milhares) na área;

Percap: renda per capita (em US\$) por domicílio com TV a cabo;

Taxa: taxa de instalação;

Custo: custo médio mensal de manutenção;

Ncabo: número de canais a cabo disponíveis na área e 
Ntv: número de canais abertos com sinal de boa qualidade disponíveis na área.

Tabela D.1.1: Assinaturas de TV a cabo

\begin{tabular}{crrrrrrr}
\hline Observação & Nass & Domic & Percap & Taxa & Custo & Ncabo & Ntv \\
\hline \hline 1 & 105,000 & 350,000 & 9839 & 14,95 & 10,00 & 16 & 13 \\
2 & 90,000 & 255,631 & 10606 & 15,00 & 7,50 & 15 & 11 \\
3 & 14,000 & 31,000 & 10455 & 15,00 & 7,00 & 11 & 9 \\
4 & 11,700 & 34,840 & 8958 & 10,00 & 7,00 & 22 & 10 \\
5 & 46,000 & 153,434 & 11741 & 25,00 & 10,00 & 20 & 12 \\
6 & 11,217 & 26,621 & 9378 & 15,00 & 7,66 & 18 & 8 \\
7 & 12,000 & 18,000 & 10433 & 15,00 & 7,50 & 12 & 8 \\
8 & 6,428 & 9,324 & 10167 & 15,00 & 7,00 & 17 & 7 \\
9 & 20,100 & 32,000 & 9218 & 10,00 & 5,60 & 10 & 8 \\
10 & 8,500 & 28,000 & 10519 & 15,00 & 6,50 & 6 & 6 \\
11 & 1,600 & 8,000 & 10025 & 17,50 & 7,50 & 8 & 6 \\
12 & 1,100 & 5,000 & 9714 & 15,00 & 8,95 & 9 & 9 \\
13 & 4,355 & 15,204 & 9294 & 10,00 & 7,00 & 7 & 7 \\
14 & 78,910 & 97,889 & 9784 & 24,95 & 9,49 & 12 & 7 \\
15 & 19,600 & 93,000 & 8173 & 20,00 & 7,50 & 9 & 7 \\
16 & 1,000 & 3,000 & 8967 & 9,95 & 10,00 & 13 & 6 \\
17 & 1,650 & 2,600 & 10133 & 25,00 & 7,55 & 6 & 5 \\
18 & 13,400 & 18,284 & 9361 & 15,50 & 6,30 & 11 & 5 \\
19 & 18,708 & 55,000 & 9085 & 15,00 & 7,00 & 16 & 6 \\
20 & 1,352 & 1,700 & 10067 & 20,00 & 5,60 & 6 & 6 \\
21 & 170,000 & 270,000 & 8908 & 15,00 & 8,75 & 15 & 5 \\
22 & 15,388 & 46,540 & 9632 & 15,00 & 8,73 & 9 & 6 \\
23 & 6,555 & 20,417 & 8995 & 5,95 & 5,95 & 10 & 6 \\
24 & 40,000 & 120,000 & 7787 & 25,00 & 6,50 & 10 & 5 \\
25 & 19,900 & 46,390 & 8890 & 15,00 & 7,50 & 9 & 7 \\
26 & 2,450 & 14,500 & 8041 & 9,95 & 6,25 & 6 & 4 \\
27 & 3,762 & 9,500 & 8605 & 20,00 & 6,50 & 6 & 5 \\
28 & 24,882 & 81,980 & 8639 & 18,00 & 7,50 & 8 & 4 \\
29 & 21,187 & 39,700 & 8781 & 20,00 & 6,00 & 9 & 4 \\
30 & 3,487 & 4,113 & 8551 & 10,00 & 6,85 & 11 & 4 \\
31 & 3,000 & 8,000 & 9306 & 10,00 & 7,95 & 9 & 6 \\
32 & 42,100 & 99,750 & 8346 & 9,95 & 5,73 & 8 & 5 \\
33 & 20,350 & 33,379 & 8803 & 15,00 & 7,50 & 8 & 4 \\
34 & 23,150 & 35,500 & 8942 & 17,50 & 6,50 & 8 & 5 \\
35 & 9,866 & 34,775 & 8591 & 15,00 & 8,25 & 11 & 4 \\
36 & 42,608 & 64,840 & 9163 & 10,00 & 6,00 & 11 & 6 \\
37 & 10,371 & 30,556 & 7683 & 20,00 & 7,50 & 8 & 6 \\
38 & 5,164 & 16,500 & 7924 & 14,95 & 6,95 & 8 & 5 \\
39 & 31,150 & 70,515 & 8454 & 9,95 & 7,00 & 10 & 4 \\
40 & 18,350 & 42,040 & 8429 & 20,00 & 7,00 & 6 & 4 \\
\hline & & & & & & &
\end{tabular}




\section{D.2 Demanda de energia elétrica}

A Tabela D.2.1 contém os dados sobre a demanda de energia elétrica, onde $D 1_{t}, D 2_{t}$ e $D 3_{t}$ são variáveis binárias relacionadas com o trimestre $t$ do ano $\mathrm{A}$ e as outras variáveis representam:

$Q_{t}$ : demanda de eletricidade agregada per capita para o setor residencial;

$P E_{t}$ : preço médio da eletricidade para o setor residencial;

$P G_{t}$ : preço do gás natural para o setor residencial e

$Y_{t}$ : renda per capita.

Tabela D.2.1: Demanda de energia elétrica.

\begin{tabular}{ccccccccc}
\hline $\mathrm{A}$ & $\mathrm{t}$ & $Q_{t}$ & $P E_{t}$ & $P G_{t}$ & $Y_{t}$ & $D 1_{t}$ & $D 2_{t}$ & $D 3_{t}$ \\
\hline \hline 1 & $\mathrm{l}$ & 0.30800536 & 7.64518690 & 2.77420998 & 0.00914456 & 1 & 0 & 0 \\
1 & 2 & 0.26834363 & 7.95841503 & 3.10906148 & 0.00923471 & 0 & 1 & 0 \\
1 & 3 & 0.27840772 & 7.92997503 & 4.04409552 & 0.00932230 & 0 & 0 & 1 \\
1 & 4 & 0.28370830 & 7.82164145 & 3.05730581 & 0.00950548 & 0 & 0 & 0 \\
2 & 1 & 0.33067492 & 7.35322905 & 2.71285081 & 0.00960076 & 1 & 0 & 0 \\
2 & 2 & 0.28388155 & 7.71690655 & 3.14473939 & 0.00966927 & 0 & 1 & 0 \\
2 & 3 & 0.30097651 & 7.64894676 & 3.47958493 & 0.00972013 & 0 & 0 & 1 \\
2 & 4 & 0.29878822 & 7.53726721 & 3.01232100 & 0.00964969 & 0 & 0 & 0 \\
3 & 1 & 0.35450837 & 7.04945183 & 2.66247821 & 0.00974009 & 1 & 0 & 0 \\
3 & 2 & 0.29236847 & 7.52932024 & 3.09602141 & 0.00984403 & 0 & 1 & 0 \\
3 & 3 & 0.32083428 & 7.37974453 & 3.95054865 & 0.00998568 & 0 & 0 & 1 \\
3 & 4 & 0.30998397 & 7.31903124 & 3.03680444 & 0.01003013 & 0 & 0 & 0 \\
4 & 1 & 0.36952662 & 6.81957054 & 2.62996173 & 0.01020502 & 1 & 0 & 0 \\
4 & 2 & 0.31365973 & 7.20112085 & 3.01820755 & 0.01028083 & 0 & 1 & 0 \\
4 & 3 & 0.35007703 & 7.02109432 & 3.96968317 & 0.01034642 & 0 & 0 & 1 \\
4 & 4 & 0.33276981 & 7.02124262 & 2.90021181 & 0.01034942 & 0 & 0 & 0 \\
5 & 1 & 0.38749585 & 6.54028463 & 2.74633431 & 0.01053808 & 1 & 0 & 0 \\
5 & 2 & 0.33387709 & 6.86014271 & 3.09525871 & 0.01066791 & 0 & 1 & 0 \\
5 & 3 & 0.36804986 & 6.66966391 & 3.92323565 & 0.01077701 & 0 & 0 & 1 \\
5 & 4 & 0.35709164 & 6.63340855 & 3.02050757 & 0.01099775 & 0 & 0 & 0 \\
6 & 1 & 0.41694346 & 6.15353727 & 2.66674948 & 0.01118029 & 1 & 0 & 0 \\
6 & 2 & 0.35326710 & 6.51159859 & 3.01723003 & 0.01119937 & 0 & 1 & 0 \\
6 & 3 & 0.40777826 & 6.27930784 & 3.81770802 & 0.01126028 & 0 & 0 & 1 \\
6 & 4 & 0.38217804 & 6.20854807 & 2.84517026 & 0.01128659 & 0 & 0 & 0 \\
7 & 1 & 0.44221917 & 5.87383795 & 2.57694674 & 0.01131980 & 1 & 0 & 0 \\
7 & 2 & 0.38583204 & 6.20719862 & 2.94127989 & 0.01137994 & 0 & 1 & 0 \\
7 & 3 & 0.42855132 & 6.06665373 & 3.66671538 & 0.01149168 & 0 & 0 & 1 \\
7 & 4 & 0.41222385 & 5.98085690 & 2.74726343 & 0.01152810 & 0 & 0 & 0 \\
8 & 1 & 0.49082169 & 5.49876261 & 2.47987032 & 0.01163357 & 1 & 0 & 0 \\
8 & 2 & 0.40941107 & 5.83722544 & 2.79997373 & 0.01180093 & 0 & 1 & 0 \\
8 & 3 & 0.48547110 & 5.61731529 & 3.45636535 & 0.01186746 & 0 & 0 & 1 \\
8 & 4 & 0.44673607 & 5.56372929 & 2.64927459 & 0.01182800 & 0 & 0 & 0
\end{tabular}




\begin{tabular}{|c|c|c|c|c|c|c|c|c|}
\hline 9 & 1 & 0.53332543 & 5.13844633 & 2.35906005 & 0.01195509 & 1 & 0 & 0 \\
\hline 9 & 2 & 0.44059545 & 5.48616648 & 2.68346119 & 0.01195672 & 0 & 1 & 0 \\
\hline 9 & 3 & 0.54803473 & 5.21186781 & 3.31664300 & 0.01198937 & 0 & 0 & 1 \\
\hline 9 & 4 & 0.49101120 & 5.22422218 & 2.56152606 & 0.01190421 & 0 & 0 & 0 \\
\hline 10 & 1 & 0.57242423 & 4.84008980 & 2.32434344 & 0.01180006 & 1 & 0 & 0 \\
\hline 10 & 2 & 0.48410484 & 5.13360834 & 2.64912558 & 0.01176797 & 0 & 1 & 0 \\
\hline 10 & 3 & 0.60302770 & 4.98096657 & 3.27019763 & 0.01186475 & 0 & 0 & 1 \\
\hline 10 & 4 & 0.52503026 & 5.08426189 & 2.55258965 & 0.01171888 & 0 & 0 & 0 \\
\hline 11 & 1 & 0.60602528 & 4.76719999 & 2.32727671 & 0.01198772 & 1 & 0 & 0 \\
\hline 11 & 2 & 0.51891249 & 5.01803827 & 2.62444520 & 0.01194521 & 0 & 1 & 0 \\
\hline 11 & 3 & 0.62209785 & 4.94619703 & 3.33343983 & 0.01198712 & 0 & 0 & 1 \\
\hline 11 & 4 & 0.5608 & 4.99 & 2.58277440 & 93268 & 0 & 0 & 0 \\
\hline 12 & 1 & 0.62708759 & 4.79266357 & 2.37980080 & 0.01218264 & 1 & 0 & 0 \\
\hline 12 & 2 & 0.54874682 & 5.09319210 & 2.68980694 & 0.01239293 & 0 & 1 & 0 \\
\hline 12 & 3 & 0.65694511 & 4.95712137 & 3.23334169 & 0.01247493 & 0 & 0 & 1 \\
\hline 12 & 4 & 0.60439968 & 4.91112804 & 2.51575303 & 0.01268085 & 0 & 0 & 0 \\
\hline 13 & 1 & 0.68328059 & 4.67283297 & 2.33333063 & 0.01294289 & 1 & 0 & 0 \\
\hline 13 & 2 & 0.57989609 & 4.94276857 & 2.67354584 & 0.01295302 & 0 & 1 & 0 \\
\hline 13 & 3 & 0.72811598 & 4.79395962 & 3.13997459 & 0.01291298 & 0 & 0 & 1 \\
\hline 13 & 4 & 0.62451297 & 4.83387899 & 2.55854464 & 0.01298187 & 0 & 0 & 0 \\
\hline 14 & I & 0.66959435 & 4.83421087 & 2.40839648 & 0.01289692 & 1 & 0 & 0 \\
\hline 14 & 2 & 0.59413171 & 5.32074070 & 2.75469518 & 0.01289350 & 0 & 1 & 0 \\
\hline 14 & 3 & 0.70640928 & 5.39235258 & 38322 & 69503 & 0 & 0 & 1 \\
\hline 14 & 4 & 0.62540507 & 5.39791536 & 2.73541474 & 0.01255311 & 0 & 0 & 0 \\
\hline 15 & 1 & 0.70960039 & 5.22349358 & 2.61702061 & 0.01228601 & 1 & 0 & 0 \\
\hline 15 & 2 & 0.62260377 & 5.44529819 & 2.95232224 & 0.01237817 & 0 & 1 & 0 \\
\hline 15 & 3 & 0.74306965 & 5.50917530 & 3.47252870 & 0.01256718 & 0 & 0 & 1 \\
\hline 15 & 4 & 0.63985091 & 5.46223164 & 31594 & 0.01269196 & 0 & 0 & 0 \\
\hline 16 & 1 & 0.74697447 & 5.23494911 & 2.91738129 & 0.01291349 & 1 & 0 & 0 \\
\hline 16 & 2 & 0.61285406 & 5.55359745 & 3.27993631 & 0.01294898 & 0 & 1 & 0 \\
\hline 16 & 3 & 0.75429350 & 5.64516401 & 3.91158652 & 0.01297108 & 0 & 0 & 1 \\
\hline 16 & 4 & 0.69813275 & 5.46667147 & 4.27899122 & 0.01306254 & 0 & 0 & 0 \\
\hline 17 & 1 & 0.81564754 & 5.30334044 & 3.27748561 & 0.01319841 & 1 & 0 & 0 \\
\hline 17 & 2 & 0.63987577 & 5.68160534 & 3.70696568 & 0.01338583 & 0 & 1 & 0 \\
\hline 17 & 3 & 0.81182355 & 5.90110493 & 4.23934031 & 0.01361182 & 0 & 0 & l \\
\hline 17 & 4 & 0.69549668 & 5.62990713 & 3.48335361 & 0.01353800 & 0 & 0 & 0 \\
\hline 18 & 1 & 0.84910756 & 5.35183573 & 3.37630939 & 0.01362886 & 1 & 0 & 0 \\
\hline 18 & 2 & 0.66610706 & 5.73035097 & 3.68710351 & 0.01401979 & 0 & 1 & 0 \\
\hline 18 & 3 & 0.82361311 & 5.77223778 & 4.21130323 & 0.01409499 & 0 & 0 & 1 \\
\hline 18 & 4 & 0.71349722 & 5.51756096 & 3.52143955 & 0.01423942 & 0 & 0 & 0 \\
\hline 19 & 1 & 0.87685442 & 5.17210197 & 4.39531507 & 0.01419568 & 1 & 0 & 0 \\
\hline 19 & 2 & 0.67969620 & 5.58356667 & 3.75331378 & 0.01415907 & 0 & 1 & 0 \\
\hline 19 & 3 & 0.81007040 & 5.78466034 & 4.43317604 & 0.01423306 & 0 & 0 & 1 \\
\hline 19 & 4 & & & & & 0 & 0 & 0 \\
\hline 20 & 1 & 0.84437078 & 5.37417889 & 3.97319126 & 0.01426184 & 1 & 0 & 0 \\
\hline 20 & 2 & 0.68406653 & 5.80723810 & 4.34946060 & 0.01389695 & 0 & 1 & 0 \\
\hline 20 & 3 & 0.89883024 & 6.06001234 & 5.06670094 & 0.01386312 & 0 & 0 & 1 \\
\hline 20 & 4 & 0.73912853 & 5.74602461 & 4.36355448 & 0.01399696 & 0 & 0 & 0 \\
\hline 21 & 1 & 0.85256535 & 5.66703844 & 4.19112778 & 0.01423567 & 1 & 0 & 0 \\
\hline 21 & 2 & & & & & 0 & 1 & 0 \\
\hline 21 & 3 & 0.88925880 & 6.57580376 & 5.15262365 & 0.01417765 & 0 & 0 & 1 \\
\hline 21 & 4 & 0.73861104 & 6.19287395 & 4.57044888 & 0.01394008 & 0 & 0 & 0 \\
\hline 22 & 1 & 0.86724007 & 6.18621683 & 4.59979963 & 0.01368745 & 1 & 0 & 0 \\
\hline 22 & 2 & 0.69785839 & 6.52221394 & 5.05689907 & 0.01369381 & 0 & l & 0 \\
\hline 22 & 3 & 0.84755844 & 6.66881037 & 5.81978750 & 0.01355230 & 0 & 0 & 1 \\
\hline 22 & 4 & & & & 0.01353536 & 0 & 0 & 0 \\
\hline 23 & 1 & 0.82811236 & 6.25222349 & 5.49710894 & 0.01362200 & 1 & 0 & 0 \\
\hline 23 & 2 & 0.68105930 & 6.60154247 & 5.79531860 & 0.01390618 & 0 & 1 & 0 \\
\hline 23 & 3 & 0.94196534 & 6.87017965 & 6.52311754 & 0.01406361 & 0 & 0 & 1 \\
\hline 23 & 4 & 0.74517667 & 6.52699089 & 5.60170937 & 0.01427785 & 0 & 0 & \\
\hline
\end{tabular}




\section{D.3 Processo infeccioso pulmonar}

A Tabela D.3.1 a seguir apresenta os dados de um estudo realizado no Hospital Heliópolis na cidade de São Paulo-SP sobre o processo infeccioso pulmonar onde a variável Resp representa o tipo de tumor apresentado pelo paciente com valores 1 para maligno e 0 para benigno. As variáveis $\mathrm{HL}$ e FF apresentam os valores 1, 2, 3 e 4 correspondendo, respectivamente, aos níveis de intensidade das células: ausente, discreta, moderada e intensa. Os níveis da variável Sexo são 1 para masculino e 2 para feminino. A variável Idade é dada em anos.

Tabela D.3.1: Processo infeccioso pulmonar.

\begin{tabular}{|c|c|c|c|c|c|c|c|c|c|c|c|}
\hline Pac. & Resp & Idade & Sexo & HL & $\mathrm{FF}$ & Pac. & Resp & Idade & Sexo & HL & $\mathrm{FF}$ \\
\hline 1 & 0 & 26 & 1 & 3 & 1 & 2 & 0 & 21 & 1 & 3 & 1 \\
\hline 3 & 0 & 45 & 1 & 3 & 3 & 4 & 0 & 19 & 2 & 4 & 3 \\
\hline 5 & 0 & 16 & 2 & 4 & 3 & 6 & 0 & 72 & 2 & 4 & 3 \\
\hline 7 & 0 & 53 & 1 & 3 & 1 & 8 & 0 & 33 & 1 & 4 & 2 \\
\hline 9 & 0 & 39 & 1 & 3 & 2 & 10 & 0 & 41 & 1 & 3 & 2 \\
\hline 11 & 0 & 26 & 2 & 3 & 3 & 12 & 0 & 27 & 2 & 3 & 3 \\
\hline 13 & 0 & 46 & 1 & 3 & 1 & 14 & 0 & 27 & 1 & 3 & 3 \\
\hline 15 & 0 & 65 & 1 & 3 & 2 & 16 & 0 & 27 & 1 & 4 & 1 \\
\hline 17 & 0 & 32 & 2 & 4 & 1 & 18 & 0 & 22 & 2 & 3 & 2 \\
\hline 19 & 0 & 23 & I & 4 & 2 & 20 & 0 & 42 & 2 & 4 & 2 \\
\hline 21 & 0 & 82 & 2 & 3 & 2 & 22 & 0 & 23 & 2 & 3 & 1 \\
\hline 23 & 0 & 55 & 1 & 3 & 2 & 24 & 0 & 43 & 1 & 3 & 1 \\
\hline 25 & 0 & 49 & 1 & 3 & 1 & 26 & 0 & 20 & 2 & 2 & 1 \\
\hline 27 & 0 & 23 & 1 & 4 & 2 & 28 & 0 & 28 & 1 & 3 & 2 \\
\hline 29 & 0 & 34 & 1 & 3 & 1 & 30 & 0 & 18 & 1 & 3 & 1 \\
\hline 31 & 0 & 22 & 1 & 3 & 2 & 32 & 0 & 50 & 2 & 3 & 3 \\
\hline 33 & 0 & 64 & 1 & 2 & 1 & 34 & 0 & 29 & 2 & 4 & 2 \\
\hline 35 & 0 & 24 & 2 & 3 & 3 & 36 & 0 & 50 & 1 & 2 & 3 \\
\hline 37 & 0 & 38 & 1 & 2 & 3 & 38 & 0 & 20 & 2 & 3 & 3 \\
\hline 39 & 0 & 44 & 1 & 3 & 3 & 40 & 0 & 59 & 1 & 3 & 3 \\
\hline 41 & 0 & 43 & 1 & 3 & 3 & 42 & 0 & 27 & 1 & 4 & 3 \\
\hline 43 & 0 & 20 & 1 & 2 & 1 & 44 & 0 & 24 & 1 & 3 & 2 \\
\hline 45 & 0 & 46 & 1 & 3 & 4 & 46 & 0 & 40 & 1 & 2 & 3 \\
\hline 47 & 0 & 21 & 2 & 3 & 3 & 48 & 0 & 21 & 1 & 3 & 2 \\
\hline 49 & 0 & 42 & 1 & 3 & 3 & 50 & 0 & 23 & 1 & 3 & 3 \\
\hline 51 & 0 & 38 & 2 & 3 & 4 & 52 & 0 & 53 & 1 & 3 & 3 \\
\hline 53 & 0 & 53 & 1 & 3 & 2 & 54 & 0 & 21 & 1 & 1 & 1 \\
\hline 55 & 0 & 57 & 1 & 3 & 1 & 56 & 0 & 63 & 2 & 3 & 1 \\
\hline 57 & 0 & 21 & 1 & 3 & 2 & 58 & 0 & 45 & 1 & 3 & 2 \\
\hline 59 & 0 & 77 & 1 & 2 & 1 & 60 & 0 & 58 & 1 & 3 & 2 \\
\hline
\end{tabular}




\begin{tabular}{|c|c|c|c|c|c|c|c|c|c|c|c|}
\hline 61 & 0 & 28 & 1 & 3 & 2 & 62 & 0 & 83 & 1 & 3 & 1 \\
\hline 63 & 0 & 22 & 1 & 2 & 1 & 64 & 0 & 36 & 1 & 2 & 1 \\
\hline 65 & 0 & 43 & 1 & 3 & 2 & 66 & 0 & 22 & 1 & 3 & 3 \\
\hline 67 & 0 & 30 & 1 & 3 & 3 & 68 & 0 & 46 & 1 & 2 & 1 \\
\hline 69 & 0 & 78 & 2 & 3 & 3 & 70 & 0 & 23 & 1 & 3 & 3 \\
\hline 71 & 0 & 56 & 1 & 2 & 1 & 72 & 0 & 56 & 1 & 3 & 3 \\
\hline 73 & 0 & 44 & 1 & 2 & 1 & 74 & 0 & 64 & 1 & 2 & 3 \\
\hline 75 & 0 & 18 & 2 & 3 & 3 & 76 & 0 & 23 & 1 & 2 & 2 \\
\hline 77 & 0 & 62 & 1 & 1 & 1 & 78 & 0 & 53 & 1 & 2 & 2 \\
\hline 79 & 0 & 23 & 1 & 3 & 1 & 80 & 0 & 23 & 1 & 3 & 1 \\
\hline 81 & 0 & 49 & 1 & 3 & 2 & 82 & 0 & 21 & 1 & 3 & 3 \\
\hline 83 & 0 & 17 & 1 & 3 & 3 & 84 & 0 & 41 & 2 & 3 & 3 \\
\hline 85 & 0 & 45 & 1 & 3 & 3 & 86 & 0 & 51 & 1 & 2 & 3 \\
\hline 87 & 0 & 62 & 2 & 3 & 1 & 88 & 0 & 48 & 1 & 2 & 2 \\
\hline 89 & 0 & 27 & 1 & 3 & 2 & 90 & 0 & 18 & 2 & 3 & 2 \\
\hline 91 & 0 & 67 & 1 & 3 & 3 & 92 & 0 & 75 & 1 & 2 & 3 \\
\hline 93 & 0 & 67 & 1 & 2 & 2 & 94 & 0 & 49 & 1 & 2 & 1 \\
\hline 95 & 0 & 63 & 1 & 3 & 2 & 96 & 0 & 87 & 1 & 2 & 1 \\
\hline 97 & 0 & 53 & 2 & 2 & 1 & 98 & 0 & 18 & 1 & 2 & 3 \\
\hline 99 & 0 & 30 & 1 & 4 & 3 & 100 & 0 & 48 & 1 & 3 & 3 \\
\hline 101 & 0 & 31 & 2 & 3 & 3 & 102 & 0 & 56 & 1 & 2 & 2 \\
\hline 103 & 0 & 48 & 1 & 3 & 2 & 104 & 0 & 33 & 2 & 4 & 2 \\
\hline 105 & 1 & 58 & 1 & 3 & 4 & 106 & 1 & 76 & 1 & 3 & 3 \\
\hline 107 & 1 & 64 & 1 & 2 & 3 & 108 & 1 & 14 & 1 & 2 & 2 \\
\hline 109 & 1 & 34 & 1 & 2 & 1 & 110 & 1 & 51 & 1 & 3 & 3 \\
\hline 111 & 1 & 60 & 1 & 3 & 3 & 112 & 1 & 73 & 1 & 4 & 2 \\
\hline 113 & 1 & 72 & 1 & 3 & 4 & 114 & 1 & 62 & 1 & 2 & 1 \\
\hline 115 & 1 & 60 & 2 & 2 & 1 & 116 & 1 & 43 & 1 & 1 & 1 \\
\hline 117 & 1 & 62 & 1 & 2 & 1 & 118 & 1 & 55 & 1 & 2 & 1 \\
\hline 119 & 1 & 58 & 2 & 2 & 1 & 120 & 1 & 45 & 1 & 2 & 2 \\
\hline 121 & 1 & 58 & 1 & 2 & 1 & 122 & 1 & 15 & 1 & 2 & 1 \\
\hline 123 & 1 & 61 & 2 & 2 & 1 & 124 & 1 & 60 & 1 & 2 & 1 \\
\hline 125 & 1 & 61 & 2 & 3 & 2 & 126 & l & 56 & 2 & 2 & 1 \\
\hline 127 & 1 & 78 & 1 & 2 & 2 & 128 & 1 & 21 & 1 & 2 & 1 \\
\hline 129 & 1 & 75 & 1 & 2 & 1 & 130 & 1 & 56 & 2 & 2 & 1 \\
\hline 131 & 1 & 73 & 2 & 2 & 1 & 132 & 1 & 56 & 1 & 2 & 2 \\
\hline 133 & 1 & 62 & 2 & 3 & 1 & 134 & 1 & 56 & 1 & 2 & 1 \\
\hline 135 & 1 & 56 & 2 & 3 & 1 & 136 & 1 & 52 & 2 & 2 & l \\
\hline 137 & 1 & 57 & 2 & 2 & 2 & 138 & 1 & 29 & 2 & 1 & 1 \\
\hline 139 & 1 & 51 & 1 & 2 & 2 & 140 & 1 & 77 & 1 & 3 & 3 \\
\hline 141 & 1 & 40 & 2 & 3 & 2 & 142 & 1 & 65 & 1 & 2 & 1 \\
\hline 143 & 1 & 60 & 1 & 2 & 2 & 144 & 1 & 69 & 2 & 3 & 1 \\
\hline 145 & 1 & 67 & 1 & 3 & 3 & 146 & 1 & 58 & 1 & 2 & 1 \\
\hline 147 & 1 & 72 & 1 & 3 & 1 & 148 & 1 & 51 & 2 & 2 & 1 \\
\hline 149 & 1 & 57 & 1 & 3 & 1 & 150 & 1 & 36 & 2 & 2 & 1 \\
\hline 151 & 1 & 58 & 1 & 3 & 3 & 152 & 1 & 59 & 1 & 3 & 1 \\
\hline 153 & 1 & 59 & 1 & 1 & 1 & 154 & 1 & 57 & 2 & 2 & 1 \\
\hline 155 & 1 & 73 & 1 & 2 & 1 & 156 & 1 & 69 & 1 & 2 & 3 \\
\hline 157 & 1 & 61 & 1 & 3 & 1 & 158 & 1 & 67 & 1 & 2 & 1 \\
\hline 159 & 1 & 70 & 1 & 2 & 2 & 160 & 1 & 64 & 1 & 2 & 2 \\
\hline 161 & 1 & 69 & 1 & 3 & 3 & 162 & 1 & 52 & 1 & 2 & 1 \\
\hline 163 & I & 59 & 1 & 3 & 1 & 164 & 1 & 50 & 2 & 2 & 1 \\
\hline 165 & 1 & 48 & 1 & 2 & 1 & 166 & 1 & 49 & 1 & 1 & 1 \\
\hline 167 & 1 & 78 & 1 & 2 & 1 & 168 & 1 & 66 & 1 & 2 & 1 \\
\hline 169 & 1 & 74 & 1 & 2 & 1 & 170 & 1 & 50 & 1 & 1 & 2 \\
\hline 171 & 1 & 75 & 2 & 1 & 2 & 172 & 1 & 55 & 2 & 3 & 3 \\
\hline 173 & 1 & 50 & 2 & 2 & 1 & 174 & 1 & 57 & 2 & 3 & 1 \\
\hline 175 & 1 & 70 & 2 & 2 & 1 & & & & & & \\
\hline
\end{tabular}




\section{D.4 Tratamento de epilepsia}

A Tabela D.4.1 apresenta os dados sobre a droga progabide no tratamento da epilepsia.

A variável Trat apresenta dois níveis com valores 0 para placebo e 1 para tratado. A variável Id é a idade dada em anos. As outras variáveis são as seguintes:

$Y_{i}$ : número de ataques epilépticos no $i$-ésimo período, $i=1,2,3,4$;

Base: número de ataques epilépticos no período considerado anterior ao tratamento.

Tabela D.4.1: Tratamento de epilepsia.

\begin{tabular}{|c|c|c|c|c|c|c|c|c|c|c|c|c|c|c|c|}
\hline Pac & $Y_{1}$ & $Y_{2}$ & $Y_{3}$ & $Y_{4}$ & Trat & base & Id & Pac & $Y_{1}$ & $Y_{2}$ & $Y_{3}$ & $Y_{4}$ & Trat & base & Id \\
\hline 104 & 5 & 3 & 3 & 3 & 0 & 11 & 31 & 106 & 3 & 5 & 3 & $\overline{c 3}$ & 0 & 11 & 30 \\
\hline 107 & 2 & 4 & 0 & 5 & 0 & 6 & 25 & 114 & 4 & 4 & 1 & 4 & 0 & 8 & 36 \\
\hline 116 & 7 & 18 & 9 & 21 & 0 & 66 & 22 & 118 & 5 & 2 & 8 & 7 & 0 & 27 & 29 \\
\hline 123 & 6 & 4 & 0 & 2 & 0 & 12 & 31 & 126 & 40 & 20 & 23 & 12 & 0 & 52 & 42 \\
\hline 130 & 5 & 6 & 6 & 5 & 0 & 23 & 37 & 135 & 14 & 13 & 6 & 0 & 0 & 10 & 28 \\
\hline 141 & 26 & 12 & 6 & 22 & 0 & 52 & 36 & 145 & 12 & 6 & 8 & 4 & 0 & 33 & 24 \\
\hline 201 & 4 & 4 & 6 & 2 & 0 & 18 & 23 & 202 & 7 & 9 & 12 & 14 & 0 & 42 & 36 \\
\hline 205 & 16 & 24 & 10 & 9 & 0 & 87 & 26 & 206 & 11 & 0 & 0 & 5 & 0 & 50 & 26 \\
\hline 210 & 0 & 0 & 3 & 3 & 0 & 18 & 28 & 213 & 37 & 29 & 28 & 29 & 0 & 111 & 31 \\
\hline 215 & 3 & 5 & 2 & 5 & 0 & 18 & 32 & 217 & 3 & 0 & 6 & 7 & 0 & 20 & 21 \\
\hline 219 & 3 & 4 & 3 & 4 & 0 & 12 & 29 & 220 & 3 & 4 & 3 & 4 & 0 & 9 & 21 \\
\hline 222 & 2 & 3 & 3 & 5 & 0 & 17 & 32 & 226 & 8 & 12 & 2 & 8 & 0 & 28 & 25 \\
\hline 227 & 18 & 24 & 76 & 25 & 0 & 55 & 30 & 230 & 2 & 1 & 2 & 1 & 0 & 9 & 40 \\
\hline 234 & 3 & 1 & 4 & 2 & 0 & 10 & 19 & 238 & 13 & 15 & 13 & 12 & 0 & 47 & 22 \\
\hline 101 & 11 & 14 & 9 & 8 & 1 & 76 & 18 & 102 & 8 & 7 & 9 & 4 & 1 & 38 & 32 \\
\hline 103 & 0 & 4 & 3 & 0 & 1 & 19 & 20 & 108 & 3 & 6 & 1 & 3 & 1 & 10 & 30 \\
\hline 110 & 2 & 6 & 7 & 4 & 1 & 19 & 18 & 111 & 4 & 3 & 1 & 3 & 1 & 24 & 24 \\
\hline 112 & 22 & 17 & 19 & 16 & 1 & 31 & 30 & 113 & 5 & 4 & 7 & 4 & 1 & 14 & 35 \\
\hline 117 & 2 & 4 & 0 & 4 & 1 & 11 & 27 & 121 & 3 & 7 & $i$ & 7 & 1 & 67 & 20 \\
\hline 122 & 4 & 18 & 2 & 5 & 1 & 41 & 22 & 124 & 2 & 1 & 1 & 0 & 1 & 7 & 28 \\
\hline 128 & 0 & 2 & 4 & 0 & 1 & 22 & 23 & 129 & 5 & 4 & 0 & 3 & 1 & 13 & 40 \\
\hline 137 & 11 & 14 & 25 & 15 & 1 & 46 & 33 & 139 & 10 & 5 & 3 & 8 & 1 & 36 & 21 \\
\hline 143 & 19 & 7 & 6 & 7 & 1 & 38 & 35 & 147 & 1 & 1 & 2 & 4 & 1 & 7 & 25 \\
\hline 203 & 6 & 10 & 8 & 8 & 1 & 36 & 26 & 204 & 2 & 1 & 0 & 0 & 1 & 11 & 25 \\
\hline 207 & 102 & 65 & 72 & 63 & 1 & 151 & 22 & 208 & 4 & 3 & 2 & 4 & 1 & 22 & 32 \\
\hline 209 & 8 & 6 & 5 & 7 & 1 & 41 & 25 & 211 & 1 & 3 & 1 & 5 & 1 & 32 & 35 \\
\hline 214 & 18 & 11 & 28 & 13 & 1 & 56 & 21 & 218 & 6 & 3 & 4 & 0 & 1 & 24 & 41 \\
\hline 221 & 3 & 5 & 4 & 3 & 1 & 16 & 32 & 225 & 1 & 23 & 19 & 8 & 1 & 22 & 26 \\
\hline 228 & 2 & 3 & 0 & 1 & 1 & 25 & 21 & 232 & 0 & 0 & 0 & 0 & 1 & 13 & 36 \\
\hline 236 & 1 & 4 & 3 & 2 & 1 & 12 & 37 & & & & & & & & \\
\hline
\end{tabular}




\section{D.5 Tratamento de pólipos}

A Tabela D.j.1 apresenta os dados do estudo sobre um suplemento de vitaminas C e E e fibra de trigo na redução de pólipos retais. A variável $\mathrm{T}$ representa o tratamento com 3 níveis, 0 para grupo placebo, 1 para o grupo tratado com vitaminas $\mathrm{C}$ e $\mathrm{E}$ e 2 para o grupo tratado com vitaminas $\mathrm{C}$ e $\mathrm{E}$ e fibra de trigo. A variável $\mathrm{S}$ representa sexo com 0 para feminino e 1 para masculino. O número de pólipos no início do tratamento, $\mathrm{N}$ e M e nas 16 visitas após o tratamento.

Tabela D.5.1: Tratamento de pólipos.

\begin{tabular}{|c|c|c|c|c|c|c|c|c|c|c|c|c|c|c|c|c|c|c|c|c|}
\hline \multirow[b]{2}{*}{$\mathrm{P}$} & \multirow[b]{2}{*}{$\mathrm{T}$} & \multirow[b]{2}{*}{$\mathrm{S}$} & \multirow[b]{2}{*}{$\mathrm{N}$} & \multirow[b]{2}{*}{ M } & \multicolumn{16}{|c|}{ Visita } \\
\hline & & & & & 1 & 2 & 3 & 4 & 5 & 6 & 7 & 8 & 9 & 10 & 11 & 12 & 13 & 14 & 15 & 16 \\
\hline 556983 & 0 & 0 & 41 & 22 & 36 & 56 & 34 & 46 & 61 & 56 & 73 & 28 & 53 & 28 & 33 & 12 & 46 & 39 & 8 & 21 \\
\hline 580274 & 2 & 1 & 4 & 6 & 2 & 2 & 0 & 1 & 1 & 1 & 1 & 0 & 0 & 0 & 0 & 0 & 2 & 0 & 0 & 0 \\
\hline 615158 & 2 & 1 & 26 & 7 & 1 & 2 & 0 & 0 & 1 & 1 & 0 & 0 & 1 & 1 & 0 & 1 & 2 & 2 & 2 & 1 \\
\hline 651719 & 2 & 0 & 15 & 10 & 13 & 6 & 12 & 6 & 12 & 8 & 6 & 6 & 3 & 6 & 3 & 9 & 8 & 4 & 9 & 10 \\
\hline 724688 & 2 & 0 & 9 & 2 & 6 & 2 & 8 & 6 & 4 & 4 & 4 & 5 & 1 & 1 & 5 & 3 & 3 & 4 & 6 & 10 \\
\hline 742589 & 1 & 1 & 6 & 8 & 15 & 9 & 4 & 4 & 2 & 6 & 3 & 4 & 4 & 6 & 4 & 2 & 5 & 7 & 2 & 7 \\
\hline 742595 & 1 & 0 & 1 & 12 & 7 & 8 & 5 & 3 & 6 & 8 & 10 & 3 & 12 & 7 & 9 & 12 & 5 & 3 & 5 & 2 \\
\hline 761380 & 0 & 1 & 2 & & 3 & 1 & 1 & 3 & 3 & 1 & 4 & 1 & 1 & 0 & 1 & 1 & 2 & 0 & 1 & 0 \\
\hline 769258 & 0 & 0 & 2 & 5 & 4 & 10 & 9 & 17 & 8 & 4 & 7 & 22 & 23 & 10 & 8 & 10 & 4 & 12 & 6 & 15 \\
\hline 771082 & 1 & 0 & 1 & 1 & 2 & 1 & 1 & 1 & 2 & 0 & 0 & 1 & 1 & 0 & 0 & 2 & 0 & 0 & 0 & 1 \\
\hline 776465 & 0 & 0 & 7 & 1 & 10 & 31 & 31 & 37 & 11 & 15 & 12 & 9 & 22 & 5 & 18 & 15 & 5 & 14 & 15 & 3 \\
\hline 797995 & 2 & 1 & 25 & 7 & 8 & 6 & 8 & 11 & 16 & 18 & 19 & 19 & 7 & 14 & 2 & 6 & 6 & 3 & 2 & . \\
\hline 799155 & 1 & 1 & 10 & 11 & 6 & 3 & 3 & 7 & 9 & 2 & 3 & 6 & 1 & 1 & 4 & 1 & 1 & 2 & 3 & 4 \\
\hline 808923 & 2 & 0 & 1 & 0 & 0 & 0 & 0 & 0 & 0 & 0 & 0 & 0 & 1 & 1 & 0 & 0 & 0 & 0 & 0 & 0 \\
\hline 811032 & 2 & 1 & 4 & 3 & 5 & 2 & 1 & 1 & 2 & 3 & 2 & 1 & 2 & 3 & 4 & 4 & 3 & 2 & 1 & 1 \\
\hline 811033 & 2 & 0 & 24 & 6 & 21 & 13 & 14 & 9 & 16 & 15 & 8 & 7 & 2 & 6 & 3 & 1 & 1 & 3 & 2 & 5 \\
\hline 811034 & 1 & 1 & 1 & 17 & 4 & 4 & 10 & 4 & 7 & 7 & 5 & 12 & 9 & 6 & 4 & 3 & . & 9 & 1 & 8 \\
\hline 811035 & 0 & 0 & 3 & 1 & 3 & 1 & 1 & 4 & 0 & 1 & 0 & 0 & 2 & 0 & 2 & 1 & 1 & 0 & 1 & 1 \\
\hline 811037 & 2 & 0 & 8 & 1 & 1 & 1 & 2 & 2 & 1 & 0 & 0 & l & 0 & 1 & 0 & 1 & 0 & 0 & 0 & 1 \\
\hline 811046 & 1 & 1 & 5 & 13 & 4 & 6 & 6 & 11 & 16 & 11 & 9 & 12 & 4 & 12 & 5 & 16 & 4 & 8 & 19 & 5 \\
\hline 811049 & 0 & 0 & 8 & 15 & 16 & 17 & 22 & 24 & 36 & 7 & 15 & 20 & 22 & 33 & 19 & 21 & 25 & 20 & 34 & 33 \\
\hline 811053 & 1 & 0 & 0 & 0 & 0 & 0 & 0 & 0 & 0 & 0 & 0 & 0 & 0 & 0 & 0 & 0 & 0 & 0 & 0 & 0 \\
\hline 811054 & 1 & 0 & 27 & 18 & 15 & 10 & 37 & 32 & 30 & 62 & 63 & 41 & 32 & 33 & 19 & 23 & 28 & 26 & 50 & 30 \\
\hline 811070 & 2 & 1 & 3 & 9 & 5 & 5 & 6 & 4 & 1 & 2 & 1 & 0 & 1 & 0 & 0 & 0 & 0 & 1 & 3 & 1 \\
\hline 811072 & 0 & 1 & 1 & 4 & 6 & 4 & 3 & 11 & 6 & 3 & 4 & 3 & 5 & 14 & 12 & 5 & 3 & 7 & 2 & 12 \\
\hline 811073 & 1 & 1 & 1 & 8 & 3 & 3 & 7 & 6 & 7 & 4 & 6 & 5 & 3 & 2 & 3 & 1 & 2 & 1 & 3 & 3 \\
\hline 811077 & 2 & 1 & 10 & 19 & 11 & 9 & 9 & 21 & 23 & 20 & 14 & 13 & 13 & 23 & 6 & 7 & 12 & 21 & 25 & 30 \\
\hline 811080 & 0 & 1 & 5 & 4 & 1 & 4 & 2 & 0 & 0 & 1 & 1 & 0 & 0 & 0 & 2 & 0 & 1 & 1 & 1 & 2 \\
\hline 811083 & 0 & 0 & 11 & 13 & 7 & 4 & 11 & 8 & 8 & 12 & 5 & 17 & 20 & 13 & 2 & 9 & 4 & 3 & 5 & 9 \\
\hline 814608 & 0 & 0 & 2 & 2 & 3 & 4 & 4 & 1 & 3 & 2 & 3 & 4 & 3 & 4 & 2 & 1 & 2 & 1 & 2 & 1 \\
\hline 814617 & 0 & 1 & 3 & 1 & 5 & 3 & 3 & 3 & 2 & 8 & 5 & . & . & 10 & . & . & . & 8 & . & . \\
\hline 814618 & 0 & 1 & 10 & 8 & 4 & 1 & 3 & 2 & 1 & 1 & 7 & 1 & 4 & 8 & 6 & 3 & 2 & 6 & 1 & 2 \\
\hline 814620 & 0 & 1 & 1 & 0 & 0 & 1 & 3 & 0 & 0 & 2 & 0 & 0 & . & 0 & 0 & 0 & 0 & 0 & 0 & 0 \\
\hline 814623 & 2 & 1 & 7 & 3 & 3 & 5 & 6 & 13 & 14 & . & . & 9 & 3 & 3 & 2 & 3 & 3 & 2 & 5 & 8 \\
\hline
\end{tabular}




\begin{tabular}{rrrrrrrrrrrrrrrrrrrrr}
814624 & 2 & 1 & 1 & 2 & 4 & 2 & 2 & 6 & 7 & 5 & 8 & 5 & 3 & 3 &. & 5 & 4 & 8 &. & 3 \\
814625 & 1 & 1 & 7 & 2 & 5 & 7 & 3 & 4 & 4 & 0 & 5 &. &. &. & 8 & 1 & 3 & 0 & 0 & 2 \\
815459 & 0 & 1 & 0 &. & 0 & 0 & 0 & 0 & 1 & 0 & 1 & 1 & 1 & 0 & 2 & 1 & 2 & 2 & 1 & 2 \\
819277 & 1 & 1 & 4 & 5 & 4 & 8 & 17 & 11 & 12 & 11 & 10 & 10 & 5 & 5 & 13 & 6 & 11 & 10 &. & 10 \\
819278 & 2 & 0 & 1 & 1 & 1 & 2 & 2 & 2 & 0 & 2 & 2 & 1 & 1 & 2 & 2 & 2 & 2 & 2 & 3 & 2 \\
819280 & 2 & 1 & 1 & 1 & 4 & 5 & 4 & 5 & 4 & 2 & 1 & 2 & 5 & 6 & 3 & 6 & 9 & 1 & 3 & 2 \\
819281 & 2 & 1 & 1 & 0 & 0 & 0 & 0 & 1 & 1 & 1 & 2 & 0 & 1 & 1 & 1 & 0 &. & 6 & 5 & 4 \\
819282 & 0 & 0 & 3 & 10 & 10 & 13 & 11 & 12 & 6 & 12 & 18 & 12 & 7 & 12 & 7 & 13 & 9 & 12 & 14 & 10 \\
819284 & 1 & 1 & 5 & 6 & 5 &. & 2 & 6 & 1 & 3 & 3 & 3 & 6 & 5 & 3 & 2 & 4 & 8 & 6 & 2 \\
823071 & 2 & 1 & 7 & 6 & 5 & 4 & 3 & 4 & 4 & 3 & 3 & 1 & 3 & 2 & 2 & 2 & 7 & 4 & 2 & 2 \\
823072 & 1 & 1 & 0 & 0 & 0 & 0 & 0 & 0 & 0 & 0 &. & 0 & 0 & 1 &. &. &. &. & 1 & 1 \\
823074 & 0 & 1 & 2 & 1 & 1 & 1 & 3 & 3 & 1 & 3 & 1 & 1 & 2 & 1 & 1 & 0 & 0 & 2 & 0 & 1 \\
823075 & 0 & 1 & 4 & 3 & 3 & 2 & 3 & 5 & 2 & 1 & 2 & 12 & 3 & 2 & 4 & 3 & 4 & 3 & 3 & 1 \\
823078 & 0 & 1 & 26 & 23 & 14 & 33 & 20 & 21 & 32 & 26 & 13 & 22 & 16 & 21 & 9 & 14 &. & 7 & 20 & 18 \\
829162 & 0 & 1 & 9 & 14 & 5 & 11 & 11 & 11 &. & 9 & 5 & 3 & 9 & 1 &. &. &. &. &. &. \\
829163 & 1 & 1 & 7 & 19 & 9 & 18 & 10 & 23 & 18 & 11 &. & 13 & 9 & 13 &. &. & 13 &. & 5 &. \\
829643 & 0 & 0 & 12 & 12 & 15 & 10 & 8 & 11 & 19 & 8 & 4 & 14 & 9 & 10 & 8 & 21 & 11 & 10 & 7 &. \\
829644 & 0 & 0 & 5 & 4 & 2 & 7 & 4 & 5 & 3 & 6 & 8 & 7 & 4 & 7 & 8 & 6 & 4 & 6 & 4 &. \\
829646 & 1 & 0 & 4 & 8 & 11 & 5 & 8 & 4 & 7 & 5 & 3 & 3 & 2 & 2 & 3 & 6 & 6 & 7 & 12 &. \\
829650 & 1 & 1 & 1 & 1 & 1 & 1 & 1 & 0 & 1 & 2 & 1 & 0 & 1 & 0 & 0 & 1 & 1 & 1 & 0 &. \\
829652 & 2 & 1 & 0 & 1 & 2 & 0 & 1 & 2 & 2 & 2 & 2 & 3 & 1 &. & 0 & 0 & 0 & 0 & 1 &. \\
829653 & 2 & 1 & 18 & 11 & 34 & 15 &. & 21 & 11 & 23 &. & 5 & 16 &. & 12 &. &. & 33 & 30 &. \\
829654 & 2 & 1 & 1 & 1 & 3 & 1 & 2 & 3 &. &. &. &. &. &. &. &. &. &. &. &. \\
829655 & 0 & 1 & 4 & 7 & 6 &. &. & 11 &. &. &. &. &. &. &. &. &. &. &. &. \\
\hline
\end{tabular}

\section{D.6 Previsão da safra de maçãs}

A Tabela D.6.1 apresenta os dados da produção de maçãs para 207 produtores na Nova Zelândia. A variável y representa o número de caixas de maçãs produzidas e $x_{i}$ representa o número de árvores na idade $i, i=1,2, \ldots, 10$.

Tabela D.6.1: Previsão da safra de maçãs.

\begin{tabular}{rrrrrrrrrrr}
\hline$y$ & $x_{2}$ & $x_{3}$ & $x_{4}$ & $x_{5}$ & $x_{6}$ & $x_{7}$ & $x_{8}$ & $x_{9}$ & $x_{10}$ & $x_{11}$ \\
\hline 181 & 0 & 354 & 480 & 0 & 0 & 0 & 0 & 0 & 344 & 0 \\
165 & 0 & 0 & 0 & 224 & 224 & 0 & 0 & 0 & 0 & 0 \\
49 & 0 & 16 & 384 & 0 & 544 & 0 & 0 & 0 & 0 & 0 \\
60 & 190 & 400 & 105 & 0 & 0 & 0 & 0 & 0 & 0 & 0 \\
139 & 0 & 0 & 470 & 0 & 0 & 0 & 0 & 0 & 0 & 0 \\
299 & 0 & 880 & 0 & 0 & 182 & 0 & 0 & 0 & 15 & 0 \\
8 & 0 & 180 & 0 & 0 & 0 & 0 & 0 & 0 & 0 & 0 \\
18 & 98 & 0 & 76 & 0 & 0 & 0 & 0 & 0 & 0 & 0 \\
97 & 0 & 500 & 0 & 0 & 0 & 0 & 0 & 0 & 0 & 0 \\
36 & 10 & 80 & 0 & 70 & 0 & 0 & 0 & 20 & 0 & 0 \\
99 & 0 & 0 & 360 & 0 & 0 & 0 & 0 & 0 & 0 & 0 \\
187 & 265 & 0 & 0 & 0 & 195 & 0 & 0 & 0 & 0 & 0 \\
134 & 0 & 0 & 0 & 134 & 41 & 35 & 0 & 0 & 0 & 0 \\
64 & 0 & 0 & 0 & 0 & 0 & 0 & 0 & 0 & 0 & 154
\end{tabular}




\begin{tabular}{|c|c|c|c|c|c|c|c|c|c|}
\hline 124 & 0 & 0 & 0 & 56 & 0 & 108 & 0 & 0 & 0 \\
\hline 24 & 0 & 240 & 0 & 0 & 102 & 0 & 0 & 0 & 0 \\
\hline 78 & 0 & 0 & 85 & 0 & 0 & 8.5 & 0 & 0 & 0 \\
\hline 177 & 0 & 0 & 0 & 0 & 0 & 0 & 50 & 66 & 0 \\
\hline 119 & 0 & 0 & 260 & 0 & 0 & 0 & 0 & 0 & 0 \\
\hline 24 & 72 & 0 & 0 & 24 & 30 & 0 & 0 & 0 & 0 \\
\hline 268 & 0 & 0 & 1324 & 0 & 0 & 0 & 0 & 0 & 0 \\
\hline 292 & 365 & 830 & 0 & 0 & 0 & 0 & 0 & 0 & 0 \\
\hline 81 & 0 & 0 & 0 & 350 & 0 & 0 & 0 & 0 & 0 \\
\hline 48 & 0 & 0 & 0 & 280 & 0 & 0 & 0 & 0 & 0 \\
\hline 132 & 0 & 0 & 221 & 216 & 0 & 0 & 0 & 0 & 0 \\
\hline 192 & 0 & 0 & 0 & 0 & 168 & 168 & 0 & 0 & 0 \\
\hline 50 & 0 & 0 & 0 & 123 & 0 & 0 & 0 & 30 & 0 \\
\hline 46 & 387 & 0 & 0 & 0 & 0 & 0 & 0 & 0 & 0 \\
\hline 168 & 2104 & 150 & 0 & 0 & 0 & 0 & 50 & 0 & 0 \\
\hline 241 & 0 & 0 & 0 & 450 & 0 & 0 & 0 & 0 & 0 \\
\hline 23 & 0 & 0 & 0 & 0 & $5 i$ & 0 & 0 & 0 & 0 \\
\hline 24 & 0 & 0 & 560 & 0 & 0 & 0 & 0 & 0 & 0 \\
\hline 38 & 0 & 0 & 0 & 200 & 0 & 0 & 0 & 0 & 0 \\
\hline 49 & 0 & 0 & 0 & 515 & 0 & 0 & 0 & 0 & 0 \\
\hline 37 & 0 & 0 & 0 & 1200 & 0 & 0 & 0 & 0 & 0 \\
\hline 24 & 0 & 0 & 0 & 40 & 0 & 0 & 0 & 0 & 0 \\
\hline 642 & 1000 & 0 & 0 & 453 & 0 & 0 & 192 & 0 & 0 \\
\hline 49 & 0 & 800 & 0 & 0 & 0 & 0 & 0 & 0 & 0 \\
\hline 379 & 100 & 0 & 0 & 660 & 0 & 0 & 0 & 0 & 0 \\
\hline 513 & 0 & 0 & 400 & 0 & 440 & 0 & 0 & 0 & 0 \\
\hline 156 & 0 & 0 & 0 & 0 & 0 & 0 & 0 & 0 & 126 \\
\hline 414 & 0 & 0 & 0 & 0 & 764 & 0 & 0 & 0 & 0 \\
\hline 146 & 0 & 0 & 0 & 352 & 0 & 0 & 0 & 0 & 0 \\
\hline 106 & 0 & 500 & 0 & 0 & 0 & 84 & 0 & 0 & 0 \\
\hline 75 & 0 & 0 & 98 & 66 & 0 & 0 & 0 & 0 & 0 \\
\hline 2433 & 0 & 500 & 0 & 0 & 0 & 0 & 2790 & 0 & 0 \\
\hline 60 & 0 & 0 & 224 & 0 & 0 & 0 & 0 & 0 & 0 \\
\hline 767 & 1449 & 0 & 1214 & 0 & 0 & 0 & 0 & 0 & 0 \\
\hline 49 & 0 & 2982 & 0 & 0 & 0 & 0 & 0 & 0 & 0 \\
\hline 24 & 640 & 800 & 800 & 0 & 0 & 0 & 0 & 0 & 0 \\
\hline 133 & 0 & 0 & 0 & 0 & 0 & 0 & 0 & 0 & 97 \\
\hline 51 & 0 & 0 & 0 & 300 & 0 & 0 & 0 & 0 & 0 \\
\hline 91 & 0 & 0 & 0 & 0 & 0 & 0 & 84 & 0 & 0 \\
\hline 54 & 0 & 0 & 0 & 304 & 0 & 0 & 0 & 0 & 0 \\
\hline 48 & 0 & 0 & 0 & 0 & 140 & 0 & 53 & 0 & 0 \\
\hline 216 & 0 & 0 & 16 & 384 & 0 & 544 & 0 & 0 & 0 \\
\hline 402 & 0 & 1156 & 226 & 0 & 0 & 0 & 0 & 0 & 0 \\
\hline 89 & 20 & 10 & 95 & 0 & 70 & 0 & 0 & 0 & 20 \\
\hline 92 & 200 & 0 & 150 & 0 & 0 & 0 & 0 & 0 & 0 \\
\hline 263 & 0 & 0 & 0 & 683 & 0 & 0 & 0 & 0 & 0 \\
\hline 143 & 237 & 0 & 0 & 85 & 0 & 0 & 85 & 0 & 0 \\
\hline 166 & 0 & 0 & 334 & 0 & 16 & 0 & 0 & 0 & 0 \\
\hline 200 & 0 & 400 & 0 & 200 & 0 & 0 & 0 & 0 & 0 \\
\hline 264 & 0 & 0 & 0 & 0 & 50 & 0 & 0 & 50 & 66 \\
\hline 19 & 60 & 50 & 44 & 0 & 0 & 0 & 0 & 0 & 0 \\
\hline 158 & 0 & 1509 & 0 & 0 & 0 & 0 & 0 & 0 & 0 \\
\hline 38 & 96 & 72 & 0 & 0 & 0 & 30 & 0 & 0 & 0 \\
\hline 301 & 0 & 150 & 0 & 0 & 350 & 0 & 0 & 0 & 0 \\
\hline 90 & 0 & 0 & 0 & 0 & 280 & 0 & 0 & 0 & 0 \\
\hline 253 & 111 & 0 & 0 & 221 & 216 & 0 & 0 & 0 & 0 \\
\hline 74 & 0 & 0 & 0 & 0 & 123 & 0 & 0 & 0 & 30 \\
\hline 116 & 0 & 387 & 0 & 0 & 0 & 0 & 0 & 0 & 0 \\
\hline 45 & 0 & 0 & 0 & 0 & 0 & 50 & 0 & 0 & 0 \\
\hline 208 & 0 & 2104 & 150 & 0 & 0 & 0 & 0 & 50 & 0 \\
\hline 46 & 0 & 400 & 0 & 0 & 0 & 0 & 0 & 0 & 0 \\
\hline
\end{tabular}




\begin{tabular}{|c|c|c|c|c|c|c|c|c|c|c|}
\hline 185 & 0 & 0 & 0 & 70 & 140 & 100 & 0 & 0 & 0 & \\
\hline 313 & 250 & 0 & 0 & 560 & 0 & 0 & 0 & 0 & 0 & \\
\hline 21 & 0 & 0 & 0 & 0 & 200 & 0 & 0 & 0 & 0 & \\
\hline 54 & 180 & 0 & 0 & 0 & 515 & 0 & 0 & 0 & 0 & \\
\hline 46 & 0 & 0 & 60 & 0 & 0 & 0 & 32 & 0 & 0 & \\
\hline 674 & 1500 & 0 & 0 & 0 & 1200 & 0 & 0 & 0 & 0 & \\
\hline 85 & 380 & 0 & 156 & 0 & 0 & 0 & 0 & 0 & 0 & \\
\hline 793 & 0 & 1000 & 0 & 0 & 453 & 0 & 0 & 192 & 0 & \\
\hline 46 & 0 & 0 & 0 & 0 & 6 & 15 & 0 & 0 & 0 & 30 \\
\hline 136 & 0 & 0 & 500 & 0 & 0 & 0 & 82 & 0 & 0 & \\
\hline 150 & 0 & 0 & 0 & 125 & 0 & 0 & 0 & 95 & 0 & \\
\hline 97 & 0 & 0 & 540 & 0 & 0 & 0 & 0 & 0 & 0 & \\
\hline 24 & 0 & 315 & 0 & 0 & 0 & 0 & 0 & 0 & 0 & \\
\hline 117 & 0 & 640 & 800 & 800 & 0 & 0 & 0 & 0 & 0 & \\
\hline 1028 & 80 & 0 & 2711 & 0 & 0 & 0 & 0 & 0 & 0 & 0 \\
\hline 70 & 0 & 800 & 0 & 0 & 0 & 0 & 0 & 0 & 0 & 0 \\
\hline 310 & 0 & 0 & 793 & 0 & 0 & 0 & 0 & 0 & 0 & 0 \\
\hline 138 & 0 & 0 & 354 & 480 & 0 & 0 & 0 & 0 & 0 & \\
\hline 263 & 0 & 1560 & 0 & 0 & 0 & 0 & 0 & 0 & 0 & 0 \\
\hline 124 & 0 & 0 & 393 & 0 & 0 & 0 & 0 & 0 & 0 & 0 \\
\hline 128 & 0 & 0 & 0 & 0 & 300 & 0 & 0 & 0 & 0 & 0 \\
\hline 381 & 0 & 45 & 450 & 0 & 0 & 136 & 0 & 34 & 0 & 0 \\
\hline 221 & 0 & 0 & 0 & 0 & 304 & 0 & 0 & 0 & 0 & 0 \\
\hline 69 & 0 & 0 & 0 & 0 & 0 & 140 & 0 & 53 & 0 & 0 \\
\hline 172 & 0 & 0 & 0 & 0 & 0 & 0 & 294 & 0 & 0 & 0 \\
\hline 298 & 0 & 0 & 0 & 0 & 500 & 0 & 0 & 0 & 0 & 0 \\
\hline 4981 & 0 & 3056 & 1376 & 4160 & 0 & 3592 & 0 & 0 & 0 & 0 \\
\hline 534 & 0 & 0 & 0 & 0 & 0 & 576 & 0 & 0 & 0 & 0 \\
\hline 17 & 200 & 118 & 0 & 0 & 0 & 80 & 0 & 0 & 0 & 0 \\
\hline 195 & 0 & 0 & 0 & 434 & 0 & 0 & 0 & 0 & 0 & 0 \\
\hline 524 & 0 & 1124 & 0 & 0 & 0 & 690 & 0 & 0 & 0 & 0 \\
\hline 332 & 0 & 0 & 265 & 0 & 0 & 0 & 195 & 0 & 0 & 0 \\
\hline 166 & 0 & 0 & 0 & 240 & 0 & 0 & 274 & 0 & 0 & 0 \\
\hline 67 & 0 & 0 & 0 & 150 & 0 & 0 & 12 & 0 & 0 & 0 \\
\hline 78 & 0 & 96 & 72 & 0 & 0 & 0 & 30 & 0 & 0 & 0 \\
\hline 98 & 0 & 0 & 0 & 0 & 0 & 123 & 0 & 0 & 0 & 30 \\
\hline 20 & 0 & 0 & 50 & 0 & 0 & 50 & 0 & 0 & 0 & 0 \\
\hline 106 & 0 & 0 & 0 & 0 & 0 & 0 & 070 & 0 & 70 & \\
\hline 311 & 0 & 0 & 0 & 0 & 0 & 496 & 0 & 0 & 0 & 0 \\
\hline 3670 & 0 & 0 & 0 & 0 & 0 & 4400 & 0 & 0 & 0 & 0 \\
\hline 258 & 0 & 0 & 240 & 0 & 40 & 140 & 0 & 0 & 0 & 0 \\
\hline 689 & 196 & 490 & 1416 & 0 & 0 & 0 & 0 & 0 & 0 & 0 \\
\hline 74 & 0 & 219 & 0 & 0 & 0 & 356 & 0 & 0 & 0 & 0 \\
\hline 104 & 0 & 0 & 0 & 0 & 0 & 200 & 0 & 0 & 0 & 0 \\
\hline 402 & 180 & 180 & 0 & 0 & 0 & 515 & 0 & 0 & 0 & 0 \\
\hline 217 & 0 & 0 & 0 & 0 & 100 & 0 & 0 & 177 & 0 & 0 \\
\hline 274 & 0 & 1500 & 0 & 0 & 0 & 1200 & 0 & 0 & 0 & 0 \\
\hline 21 & 0 & 0 & 0 & 0 & 0 & 40 & 0 & 0 & 0 & 0 \\
\hline 178 & 300 & 380 & 0 & 156 & 0 & 0 & 0 & 0 & 0 & 0 \\
\hline 164 & 0 & 0 & 0 & 0 & 119 & 0 & 0 & 0 & 113 & 0 \\
\hline 2000 & 0 & 0 & 12 & 0 & 290 & 1662 & 996 & 0 & 0 & 0 \\
\hline 313 & 0 & 0 & 200 & 0 & 0 & 0 & 0 & 200 & 0 & 0 \\
\hline 234 & 0 & 0 & 0 & 126 & 0 & 0 & 0 & 0 & 0 & 126 \\
\hline 386 & 318 & 150 & 0 & 0 & 0 & 352 & 0 & 0 & 0 & 0 \\
\hline 294 & 0 & 0 & 0 & 0 & 583 & 0 & 0 & 0 & 0 & 0 \\
\hline 95 & 0 & 50 & 0 & 350 & 0 & 325 & 0 & 0 & 0 & 0 \\
\hline 321 & 0 & 0 & 0 & 0 & 0 & 560 & 0 & 0 & 0 & 0 \\
\hline 895 & 0 & 0 & 0 & 850 & 0 & 0 & 1491 & 0 & 0 & 0 \\
\hline 25 & 0 & 165 & 43 & 0 & 0 & 0 & 0 & 0 & 0 & 0 \\
\hline 522 & 0 & 0 & 640 & 800 & 800 & 0 & 0 & 0 & 0 & 0 \\
\hline 69 & 0 & 0 & 0 & 0 & 0 & 0 & 0 & 0 & 0 & 97 \\
\hline
\end{tabular}




\begin{tabular}{|c|c|c|c|c|c|c|c|c|c|c|}
\hline 1078 & 0 & 0 & 0 & 0 & 1485 & 0 & 0 & 0 & 0 & 0 \\
\hline 49 & 195 & 0 & 450 & 0 & 0 & 0 & 0 & 0 & 0 & 0 \\
\hline 911 & 942 & 0 & 1698 & 0 & 0 & 0 & 0 & 0 & 0 & 0 \\
\hline 970 & 621 & 0 & 8958 & 2329 & 0 & 0 & 0 & 0 & 0 & 0 \\
\hline 32 & 0 & 0 & 220 & 0 & 0 & 0 & 0 & 0 & 0 & 0 \\
\hline 193 & 0 & 0 & 0 & 650 & 0 & 0 & 0 & 0 & 0 & 0 \\
\hline 370 & 0 & 0 & 895 & 0 & 361 & 0 & 0 & 0 & 0 & 0 \\
\hline 221 & 0 & 765 & 0 & 0 & 0 & 0 & 0 & 0 & 0 & 0 \\
\hline 32 & 11421 & 19874 & 0 & 0 & 0 & 0 & 0 & 0 & 0 & 0 \\
\hline 365 & 0 & 0 & 44 & 718 & 0 & 0 & 0 & 0 & 0 & 0 \\
\hline 26 & 390 & 372 & 0 & 0 & 0 & 0 & 0 & 0 & 0 & 0 \\
\hline 119 & 0 & 0 & 1089 & 0 & 0 & 0 & 0 & 0 & 0 & 0 \\
\hline 24 & 266 & 106 & 106 & 0 & 0 & 0 & 0 & 0 & 0 & 0 \\
\hline 16 & 0 & 0 & 0 & 0 & 45 & 0 & 0 & 0 & 0 & 0 \\
\hline 169 & 0 & 0 & 0 & 156 & 0 & 0 & 0 & 0 & 90 & 0 \\
\hline 195 & 0 & 0 & 0 & 0 & 0 & 300 & 0 & 0 & 0 & 0 \\
\hline 220 & 0 & 0 & 0 & 0 & 0 & 0 & 140 & 0 & 53 & 0 \\
\hline 160 & 0 & 0 & 0 & 0 & 0 & 0 & 0 & 294 & 0 & 0 \\
\hline 792 & 0 & 0 & 4239 & 0 & 0 & 0 & 0 & 0 & 0 & 0 \\
\hline 454 & 0 & 0 & 0 & 0 & 0 & 500 & 0 & 0 & 0 & 0 \\
\hline 84 & 0 & 200 & 118 & 0 & 0 & 0 & 80 & 0 & 0 & 0 \\
\hline 316 & 0 & 0 & 0 & 0 & 434 & 0 & 0 & 0 & 0 & 0 \\
\hline 33 & 0 & 0 & 262 & 0 & 0 & 0 & 0 & 0 & 0 & 0 \\
\hline 400 & 0 & 20 & 0 & 0 & 0 & 1080 & 0 & 0 & 0 & 0 \\
\hline 62 & 0 & 210 & 350 & 0 & 0 & 0 & 0 & 0 & 0 & 0 \\
\hline 157 & 0 & 308 & 962 & 0 & 0 & 0 & 0 & 0 & 0 & 0 \\
\hline 239 & 1400 & 0 & 0 & 0 & 150 & 0 & 0 & 0 & 0 & 40 \\
\hline 59 & 0 & 0 & 232 & 0 & 0 & 0 & 0 & 0 & 0 & 0 \\
\hline 103 & 0 & 0 & 0 & 0 & 150 & 0 & 0 & 12 & 0 & 0 \\
\hline 263 & 1600 & 130 & 0 & 0 & 0 & 0 & 60 & 0 & 0 & 0 \\
\hline 111 & 0 & 0 & 96 & 72 & 0 & 0 & 0 & 30 & 0 & 0 \\
\hline 148 & 114 & 700 & 0 & 150 & 0 & 0 & 350 & 0 & 0 & 0 \\
\hline 130 & 0 & 0 & 0 & 0 & 0 & 0 & 280 & 0 & 0 & 0 \\
\hline 448 & 159 & 0 & 111 & 0 & 0 & 221 & 216 & 0 & 0 & 0 \\
\hline 49 & 54 & 0 & 0 & 50 & 0 & 0 & 50 & 0 & 0 & 0 \\
\hline 283 & 224 & 150 & 0 & 150 & 0 & 0 & 0 & 0 & 465 & 0 \\
\hline 245 & 448 & 0 & 0 & 0 & 0 & 0 & 0 & 180 & 0 & 0 \\
\hline 133 & 0 & 0 & 423 & 144 & 0 & 0 & 0 & 0 & 0 & 0 \\
\hline 161 & 0 & 0 & 237 & 0 & 0 & 85 & 0 & 2 & 85 & 0 \\
\hline 104 & 0 & 0 & 0 & 0 & 0 & 0 & 200 & 0 & 0 & 0 \\
\hline 679 & 0 & 180 & 180 & 0 & 0 & 0 & 515 & 0 & 0 & 0 \\
\hline 11 & 0 & 0 & 0 & 75 & 0 & 0 & 75 & 0 & 0 & 0 \\
\hline 39 & 0 & 0 & 0 & 0 & 0 & 0 & 40 & 0 & 0 & 0 \\
\hline 204 & 0 & 0 & 0 & 0 & 340 & 61 & 224 & 0 & 0 & 0 \\
\hline 2 & 0 & 159 & 0 & 0 & 0 & 0 & 0 & 0 & 0 & 0 \\
\hline 944 & 600 & 536 & 2372 & 0 & 0 & 0 & 0 & 0 & 0 & 0 \\
\hline 72 & 0 & 0 & 50 & 0 & 350 & 0 & 325 & 0 & 0 & 0 \\
\hline 145 & 0 & 0 & 0 & 250 & 0 & 0 & 0 & 0 & 0 & 0 \\
\hline 23 & 0 & 20 & 0 & 12 & 0 & 0 & 0 & 97 & 0 & 0 \\
\hline 300 & 100 & 0 & 0 & 640 & 0 & 0 & 0 & 0 & 0 & 0 \\
\hline 24 & 0 & 120 & 0 & 0 & 0 & 0 & 0 & 0 & 0 & 0 \\
\hline 141 & 0 & 1850 & 570 & 0 & 0 & 0 & 0 & 0 & 0 & 0 \\
\hline 44 & 153 & 0 & 165 & 43 & 0 & 0 & 0 & 0 & 0 & 0 \\
\hline 268 & 0 & 544 & 0 & 315 & 0 & 0 & 0 & 0 & 0 & 0 \\
\hline 1344 & 0 & 0 & 0 & 640 & 800 & 800 & 0 & 0 & 0 & 0 \\
\hline 278 & 0 & 195 & 0 & 450 & 0 & 0 & 0 & 0 & 0 & 0 \\
\hline 1282 & 0 & 942 & 0 & 1698 & 0 & 0 & 0 & 0 & 0 & 0 \\
\hline 901 & 200 & 0 & 0 & 895 & 0 & 361 & 0 & 0 & 0 & 0 \\
\hline 43 & 0 & 0 & 0 & 93 & 0 & 103 & 0 & 0 & 35 & 0 \\
\hline 144 & 0 & 200 & 943 & 0 & 0 & 0 & 0 & 0 & 0 & 0 \\
\hline 4003 & 1130 & 11421 & 19874 & 0 & 0 & 0 & 0 & 0 & 0 & 0 \\
\hline
\end{tabular}




\begin{tabular}{rrrrrrrrrrr}
451 & 0 & 0 & 600 & 600 & 0 & 0 & 0 & 0 & 0 & 0 \\
776 & 0 & 402 & 0 & 0 & 296 & 480 & 0 & 0 & 0 & 0 \\
41 & 0 & 0 & 0 & 0 & 0 & 0 & 0 & 0 & 88 & 10 \\
437 & 345 & 0 & 0 & 826 & 0 & 0 & 0 & 0 & 0 & 0 \\
222 & 0 & 390 & 372 & 0 & 0 & 0 & 0 & 0 & 0 & 0 \\
121 & 280 & 0 & 350 & 0 & 0 & 0 & 0 & 0 & 0 & 0 \\
72 & 0 & 0 & 606 & 0 & 0 & 0 & 0 & 0 & 0 & 0 \\
19 & 160 & 266 & 106 & 106 & 0 & 0 & 0 & 0 & 0 & 0 \\
1996 & 4372 & 2000 & 1960 & 0 & 0 & 0 & 0 & 0 & 0 & 0 \\
22 & 0 & 0 & 96 & 0 & 0 & 0 & 0 & 0 & 0 & 0 \\
\hline
\end{tabular}




\section{Bibliografia}

Artes, R. (1998). Extensões da Teoria das Equações de Estimação Generalizadas a Dados Circulares e Modelos de Dispersão. Tese de doutorado, Instituto de Matemática e Estatística - USP, São Paulo - SP.

Avriel, M. (1976). Nonlinear Programming: Analysis and Methods. Prentice-Hall, Englewood Cliffs, NJ.

Bartholomew, D. J. (1959a). A test of homogeneity for ordered alternatives. Biometrika 46, 36-48.

Bartholomew, D. J. (1959b). A test of homogeneity for ordered alternatives II. Biometrika 46, 328-335.

Bartholomew, D. J. (1961a). A test of homogeneity of means under restricted alternatives. Journal of the Royal Statistical Society Series B 23, 239-281.

Bartholomew, D. J. (1961b). Ordered tests in the analysis of variance. Biometrika 48, $325-332$.

Bazaraa, M. S., Sherali, H. D. e Shetty, C. M. (1993). Nonlinear Programming: theory and algorithms. Second Edition. John Wiley, New York.

Berndt, E. R. e Savin, N. E. (1977). Conflict among criterion for testing hypotheses in the multivariate linear regression model. Econometrica 45, 1263-1278.

Bohrer, R. e Chow, W. (1978). Weights for one-sided multivariate inference (Algorithm AS122). Applied Statistics 27, 100-104.

Breslow, N. E. e Clayton, D. G. (1993). Approximate inference in generalized linear mixed models. Journal of the American Statistical Association 88, 9-25.

Breusch, T. S. (1979). Conflit among criterion for testing hypotheses: Extensions and comments. Econometrica 47, 203-206. 
Chen, M.-H. e Deely, J. J. (1996). Bayesian analysis for a constrained linear multiple regression problem for predicting the new crop of apples. Journal of Agricultural, Biological, and Environmental Statistics 4, 467-489.

Childs, D. P. (1967). Reduction of the multivariate normal integral to characteristic form. Biometrika 54, 293-300.

Cordeiro, G. M. e McCullagh, P. (1991). Bias correction in generalized linear models. Journal of the Royal Statistical Society Series B 53, 629-643.

Cordeiro, G. II. e Paula, G. A. (1989). Improved likelihood ratio statistics for exponential family nonlinear models. Biometrika 76, 93-100.

Cutler, J. A., Follmann, D., Elliott, P. e Suh, I. (1991). An overview of randomized trials of sodium reduction and blood pressure. Supplement I Hypertension 17, 27-33.

Diggle, P. J., Liang, K.-Y. e Zeger, S. L. (1994). Analysis of Longitudinal Data.. Oxford University Press, Oxford.

Fahrmeir, L. e Kaufmann, H. (1985). Consistency and assymptotic normality of the maximum likelihood estimator in generalized linear models. The Annals of Statistics 13, 342-368.

Fahrmeir, L. e Klinger, J. (1994). Estimating and testing generalized linear models under inequality restrictions. Statistical Papers 35, 211-229.

Feder, P. I. (1968). On the distribution of the log likelihood ratio test when the true parameter is "near" the boundaries of the hypothesis region. The Annals of Mathematical Statistics 39, 2044-2055.

Follmann, D. (1996). A simple multivariate test for one-sided altenatives. Journal of the American Statistical Association 91, 854-861.

Gouriéroux, C. e Monfort, A. (1995). Statistics and Econometric. Volume 2. Cambridge University Press, Cambridge.

Gouriéroux, C., Holly, A. e Monfort, A. (1982). Likelihood ratio tets, Wald test, and Kuhn-Tuker test in linear models with inequality constraints on the regression parameters. Econometrica 50, 63-80.

Hadgu, A. e Koch, G. (1999). Application of generalized estimating equations to a dental randomized clinical trial. Journal of Biopharmaceutical Statistics 9, 161-178.

Hastie, T. e Tibshirani, R. (1990). Generalized Additive Models. Chapman and Hall, London. 
Jørgensen, B. (1983). Maximun likelihood estimation and large-sample inference for generalized linear and nonlinear regression models. Biometrika 70, 19-28.

Jørgensen, B. (1987). Exponential dispersion models. Journal of the Royal Statistical Society Series B 49, 127-162. (with discussion).

Jørgensen, B. (1996). The Theory of Dispersion Models. Chapman and Hall, London.

Judge, G. G. e Yancey, T. A. (1986). Improved Methods of Inference in Econometrics. North-Holland, Amsterdan.

Kendall, M. G. (1941). Proof of relations connected with the tetrachoric series and its generalizations. Biometrika 32, 196-198.

Koch, G. C., Landis, J. R., Freeman, J. L., Freeman, D. H. e Lehman, G. G. (1977). A general methodology for the analysis of repeated measurements of categorical data. Biometrics 33, 133-158.

Kodde, D. A. e Palm, F. C. (1986). Wald criteria for jointly testing equality and inequality restrictions. Econometrica 54, 1243-1248.

Kudô, A. (1963). A multivariate analogue of the one-sided test. Biometrika 50, 403-418.

Laird, M. N. e Ware, J. H. (1982). Random effects models for longitudinal data. Biometrics 38, 963-974.

Lee, Y. e Nelder, J. A. (1996). Hierarchical generalized linear models. Journal of the Royal Statistical Society Series B 58, 619-678. (with discussion).

Lehmann, E. L. (1986). Testing Statistical Hypotheses. Second Edition. Springer-Verlag, New York.

Liang, K.-Y. e Zeger, S. L. (1986). Longitudinal analysis using generalized linear models. Biometrika 73, 13-22.

Liew, C. K. (1976). Inequality constrained least-squares estimation. Journal of the American Statistical Association 71, 746-751.

Luenberger, D. G. (1969). Optimization by Vector Space Methods. John Wiley, New York.

McCullagh, P. e Nelder, J. A. (1989). Generalized Linear Models. Second Edition. Chapman and Hall, London. 
McDonald, J. W. e Diamond, I. D. (1990). On the fitting of generalized linear models with nonnegative parameter constraints. Biometrics 46, 201-206.

Moore, S. D. e McCabe, P. G. (1993). Introduction of the Practice of Statistics. Freeman, New York.

Nelder, J. A. e Wedderburn, R. W. M. (1972). Generalized linear models. Journal of the Royal Statistical Society Series A 135, 370-384.

Nüesch, P. E. (1966). On the problem of testing lacation in multivariate populations for restricted alternatives. The Annals of Statistics 37, 113-119.

Neter, J., Kutner, M. H., Nachtsheim, C. J. e Wasserman, W. (1996). Applied Linear Statistical Models. Fourth Edition. Chapman and Hall, Chicago: Irwin.

Nyquist, H. (1991). Restricted estimation of generalized linear models. Applied Statistics 40, 133-141.

Ochi, Y. e Prentice, R. L. (1984). Likelihood inference in correlated probit regression. Biometrika 71, 531-543.

- Park, C. G. e Shin, D. W. (1998). An algorithm for generating correlated random variables in a class of infinitely divisible distributions. Journal of Statistical Computation and Simulation 61, 127-139.

- Park, C. G., Park, T. e Shin, D. W. (1996). A simple method for generating correlated binary variates. The American Statistician 50, 306-310.

Park, T., Shin, D. W. e Park, C. G. (1998). A generalized estimating equations approach for testing ordered group effects with repeated measurements. Biometrics 54, 16451653.

Paula, G. A. (1993). Assessing local influence in restricted regression models. Computational Statistics and Data Analysis 16, 63-79.

Paula, G. A. (1995). Influence and residual in generalized linear models. Journal of Statistical Computation and Simulation 51, 315-331.

Paula, G. A. (1997). Estimação e Testes em Modelos de Regressão com Parâmetros Restritos. ABE - 5a Escola de Modelos de Regressão, Campos do Jordão - SP.

Paula, G. A. (1999). One-sided test in generalized linear dose-response models. Computational Statistics and Data Analysis 30, 413-427. 
Paula. G. A. e Bolfarine, H. (1997). Some results on the slope of the linear regression model for the analysis of pretest-postest data. Relatório Ténico RT-MAE 9716. Instituto de Matemática e Estatística - USP, São Paulo - SP.

Paula. G. A. e Rojas, O. V. (1997). On restricted hypotheses in extreme value regression models. Computational Statistics and Data Analysis 25, 143-157.

Paula. G. A. e Sen, P. K. (1995). One-sided tests in generalized linear models with parallel regression lines. Biometrics 51, 1494-1501.

Paula. G. A. e Tuder, R. M. (1986). Utilização da regressão logística para aperfeiçoar o diagnóstico do processo infeccioso pulmonar. Revista Ciência e Cultura 38, 10461050 .

Perlman, M. D. (1969). One-sided testing problems in multivariate analysis. The Annals of Mathematical Statistics 40, 549-567.

Piegorsch, W. W. (1990). One-sided significance tests for generalized linear models under dichotomous response. Biometrics 46, 309-316.

Ramanathan, R. (1993). Statistical Methods in Econometrics. Academic Press, New York.

Rao, C. R. (1973). Linear Statistical Inference and Its Applications. Second Edition. John Wiley, New York.

Robertson, T., Wright, F. T. e Dykstra, R. L. (1988). Order Restricted Statistical Inference. John Wiley, New York.

Russek-Cohen, E. e Simon, R. M. (1993). Qualitative interactions in multifactor studies. Biometrics 49, 467-477.

SAS Intitute Inc. (1988). SAS/IML TM User's Guide, Release 6.03 Edition. SAS Institute Inc., Cary, NC. 357 pp.

Savin, N. E. (1976). Conflict among testing procedures in a linear regression model with autoregressive disturbance. Econometrica 44, 1303-1315.

Sen, P. K. e Singer, J. M. (1993). Large Sample Methods in Statistics: An Introduction with Applications. Chapman and Hall, New York.

Shapiro, A. (1985). Asymptotic distribution of test statistics in the analysis of moment structures under inequality constraints. Biometrika 72, 133-144. 
Shapiro, A. (1988). Towards a unified theory of inequality constrained testing in multivariate analysis. International Statistical Review 56, 49-62.

Silvapulle, M. J. (1994). On tests against one-sided hypoteses in some generalized linear models. Biometrics 50, 853-858.

Stiratelli, R., Laird, N. M. e Ware, J. H. (1984). Random effects models for serial observations with binary responses. Biometrics 40, 961-971.

Stukel, T. A. (1993). Comparison of methods for the analysis of longitudinal interval count data. Statistics in Medicine 12, 1339-1351.

Sun, H. J. (1988a). A fortran subroutine for computing normal orthant probabilities. Communications in Statistics, Simula 17, 1097-1111.

Sun, H. J. (1988b). A general reduction method for n-variate normal orthant probability. Communications in Statistics, Theory and Methods 17, 3913-3921.

Theil, H. (1971). Principles of Econometrics. John Wiley, New York.

Theil, H. e Van de Panne, C. (1960). Quadratic programming as an extension of classical quadratic maximization. Management Science 7, 1-20.

Ware, J. H. (1985). Linear models for the analysis of longitudinal studies. The American Statistician 39, 95-101.

Wedderburn, R. W. M. (1974). Quasi-likelihood funtions, generalized linear models and the gauss-newton method. Biometrika 61, 439-447.

Wedderburn, R. W. M. (1976). On the existence and uniqueness of the maximum likelihood estimates for certain generalized linear models. Biometrika 63, 27-32.

Wolak, F. A. (1987). An Exact test for multiple inequality and equality constraints in the linear regression model. Journal of the American Statistical Association 82, 782-793.

Wolak, F. A. (1988). Duality in testing multivariate hypotheses. Biometrika 75, 611615 .

Wolak, F. A. (1989). Testing inequality constraints in linear econometric models. Journal of Econometrics 41, 205-235.

Wolak, F. A. (1991). The local nature of hypothesis tests involving inequality constraints in nonlinear models. Econometrica 59, 981-995. 
Zeger, S. L., Liang, K.-Y. e Self. S. G. (1985). The analysis of binary longitudinal data with time independent covariates. Biometrika 72, 31-38. 